\title{
Sobolev spaces in extended metric-measure spaces
}

\author{
Giuseppe Savaré \\ Università di Pavia, Dipartimento di Matematica "F. Casorati" \\ email: giuseppe.savare@unipv.it
}

November 12, 2019

\begin{abstract}
These lecture notes contain an extended version of the material presented in the C.I.M.E. summer course in 2017. The aim is to give a detailed introduction to the metric Sobolev theory.

The notes are divided in four main parts. The first one is devoted to a preliminary and detailed study of the underlying topological, metric, and measure-theoretic aspects needed for the development of the theory in a general extended metric-topological measure space $\mathbb{X}=(X, \tau, \mathrm{d}, \mathfrak{m})$.

The second part is devoted to the construction of the Cheeger energy, initially defined on a distinguished unital algebra $\mathscr{A}$ of bounded, $\tau$-continuous and d-Lipschitz functions.

The third part deals with the basic tools needed for the dual characterization of the Sobolev spaces: the notion of $p$-Modulus of a collection of (nonparametric) rectifiable arcs and its duality with the class of nonparametric dynamic plans, i.e. Radon measures on the space of rectifiable arcs with finite $q$-barycentric entropy with respect to $\mathfrak{m}$.

The final part of the notes is devoted to the dual/weak formulation of the Sobolev spaces $W^{1, p}(\mathbb{X})$ in terms of nonparametric dynamic plans and to their relations with the Newtonian spaces $N^{1, p}(\mathbb{X})$ and with the spaces $H^{1, p}(\mathbb{X})$ obtained by the Cheeger construction. In particular, when $(X, \mathrm{~d})$ is complete, a new proof of the equivalence between these different approaches is given by a direct duality argument.

A substantial part of these Lecture notes relies on well established theories. New contributions concern the extended metric setting, the role of general compatible algebras of Lipschitz functions and their density w.r.t. the Sobolev energy, a general embedding/compactification trick, the study of reflexivity and infinitesimal Hilbertianity inherited from the underlying space, and the use of nonparametric dynamic plans for the definition of weak upper gradients.
\end{abstract}

\section{Contents}

1 Introduction $\quad 4$

1.1 Main notation . . . . . . . . . . . . . . . . . . . . . 9

I Topological and Metric-Measure structures $\quad 10$

2 Metric-measure structures $\quad 10$

2.1 Topological and measure theoretic notions . . . . . . . . . . . . . . . . . . 10

2.2 Extended metric-topological (measure) spaces . . . . . . . . . . . . . . . 12

2.3 Examples . . . . . . . . . . . . . . . . . . . . . 16

2.4 The Kantorovich-Rubinstein distance $\ldots \ldots \ldots \ldots \ldots$ 
2.5 The asymptotic Lipschitz constant . . . . . . . . . . . . . . . . . . . 19

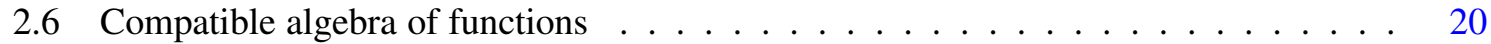

2.7 Embeddings and compactification of extended metric-measure spaces . . . . . . . 25

2.8 Notes . . . . . . . . . . . . . . . . . . . . . . . . . . 31

3 Continuous curves and nonparametric arcs 32

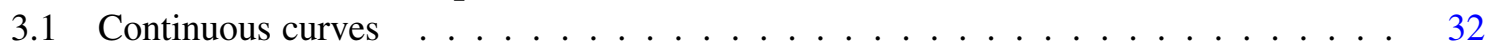

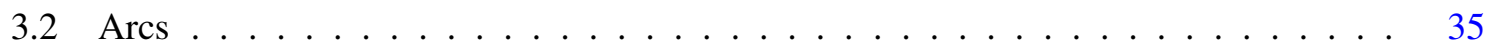

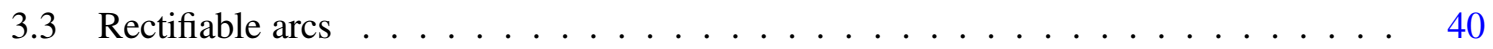

3.4 Notes . . . . . . . . . . . . . . . . . . . . . 46

4 Length and conformal distances 4

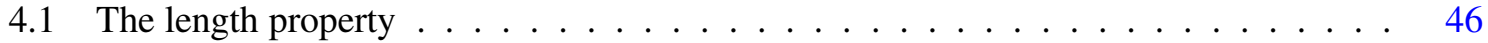

4.2 Conformal distances . . . . . . . . . . . . . . . . . . 47

4.3 Duality for Kantorovich-Rubinstein cost functionals induced by conformal distances $\quad 52$

4.4 Notes . . . . . . . . . . . . . . . . . . . . . . . . 52

II The Cheeger energy $\quad 52$

5 The strongest form of the Cheeger energy $\quad 53$

5.1 Relaxed gradients and local representation of the Cheeger energy . . . . . . . . . . 54

5.2 Invariance w.r.t. restriction and completion . . . . . . . . . . . . . 59

5.3 Notes . . . . . . . . . . . . . . . . . . . . . 61

6 Invariance of the Cheeger energy with respect to the core algebra: the compact case 61

6.1 The metric Hopf-Lax flow in compact spaces . . . . . . . . . . . . . . . 62

6.2 Invariance of the Cheeger energy with respect to $\mathscr{A}$ when $(X, \tau)$ is compact . . . . 67

6.3 Notes . . . . . . . . . . . . . . . . . . . . . . . . . . 69

III $p$-Modulus and nonparametric dynamic plans $\quad 70$

$7 \quad p$-Modulus of a family of measures and of a family of rectifiable arcs $\quad 70$

$7.1 p$-Modulus of a family of Radon measures . . . . . . . . . . . . . . 70

$7.2 p$-Modulus of a family of rectifiable $\operatorname{arcs} \ldots \ldots \ldots \ldots \ldots$

7.3 Notes . . . . . . . . . . . . . . . . . . . 75

8 (Nonparametric) Dynamic plans with barycenter in $L^{q}(X, \mathfrak{m}) \quad 75$

8.1 Notes . . . . . . . . . . . . . . . . . . . . . . . . . . . 79

9 Equivalence between $\operatorname{Cont}_{p}$ and $\operatorname{Mod}_{p} \quad \mathbf{8 0}$

9.1 Notes . . . . . . . . . . . . . . . . . . . . . . 81

IV Weak upper gradients and identification of Sobolev spaces $\quad 82$ 
10 (Nonparametric) Weak upper gradients and weak Sobolev spaces $\quad 82$

$10.1 \mathcal{T}_{q}$-test plans and $\mathcal{T}_{q}$-weak upper gradients $\ldots \ldots \ldots \ldots \ldots \ldots \ldots$

10.2 The link with $\operatorname{Mod}_{p}$-weak upper gradients . . . . . . . . . . . . . . 88

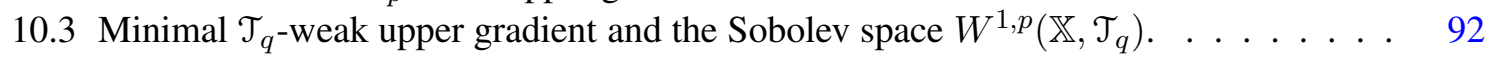

10.4 Invariance properties of weak Sobolev spaces . . . . . . . . . . . . . . . . . . 95

10.5 The approach by parametric dynamic plans . . . . . . . . . . . . . . . 98

10.6 Notes . . . . . . . . . . . . . . . . . . . . . 101

11 Identification of Sobolev spaces $\quad 101$

11.1 Dual Cheeger energies . . . . . . . . . . . . . . . . . . . 102

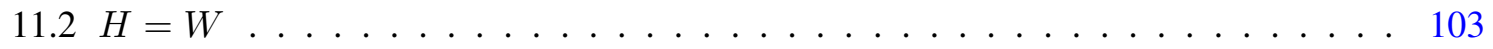

11.3 Notes . . . . . . . . . . . . . . . . . . . . 108

12 Examples and applications $\quad 108$

12.1 Refined invariance of the (strong) Cheeger energy . . . . . . . . . . . . . . . . . 108

12.2 Examples . . . . . . . . . . . . . . . . . . . . . 111

12.3 Distinguished representations of metric Sobolev spaces . . . . . . . . . . . 115

A Appendix 115

A.1 Nets . . . . . . . . . . . . . . . . . . . . . 115

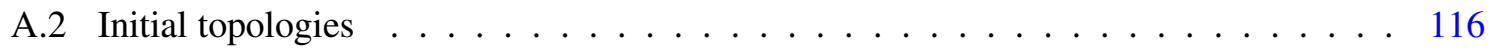

A.3 Polish, Lusin, Souslin and Analytic sets. . . . . . . . . . . . . 116

A.4 Choquet capacities . . . . . . . . . . . . . . . . . . . . . 118

A.5 Measurable maps with values in separable Banach spaces . . . . . . . . . . . . . 118

A.6 Homogeneous convex functionals . . . . . . . . . . . . . . . . . . . . . . . . . . . . . . . . . . . . .

A.7 Von Neumann theorem . . . . . . . . . . . . . . . . . . 120

$\begin{array}{ll}\text { References } & 121\end{array}$ 


\section{Introduction}

These lecture notes contain an extended version of the material presented in the C.I.M.E. summer course in 2017. The aim is to give a detailed introduction to the metric Sobolev theory, trying to unify at least two of the main approaches leading to the construction of the Sobolev spaces in general metric-measure spaces.

The notes are divided in four main parts. The first one is devoted to a preliminary and detailed study of the underlying topological, metric, and measure-theoretic aspects needed for the development of the general theory. In order to cover a wide class of examples, including genuinely infinite dimensional cases, we consider a general extended metric-topological measure space $[3] \mathbb{X}=(X, \tau, \mathrm{d}, \mathfrak{m})$, where $\mathrm{d}$ is an extended distance on $X, \tau$ is an auxiliary weaker topology compatible with $\mathrm{d}$ and $\mathfrak{m}$ is a Radon measure in $(X, \tau)$. The simplest example is a complete and separable metric space $(X, \mathrm{~d})$ where $\tau$ is the topology induced by the distance, but more general situations as duals of separable Banach spaces or Wiener spaces can be included as well.

The use of an auxiliary weaker (usually Polish or Souslin) topology $\tau$ has many technical advantages: first of all, it is easier to check the Radon property of the finite Borel measure $\mathfrak{m}$, one of our crucial structural assumptions. A second advantage is to add more flexibility in the choice of well behaved sub-algebras of Lipschitz functions and to allow for a powerful compactification method. As a reward, roughly speaking, many results which can be proved for a compact topology, can be extended to the case of a complete metric space $(X, \mathrm{~d})$ without too much effort. Therefore, for a first reading, it would not be too restrictive to assume compactness of the underlying topology, in order to avoid cumbersome technicalities.

The first part also includes a careful analysis of the topological-metric properties of the path space (Section 3) in particular concerning invariant properties with respect to parametrizations. We first recall the compact-open topology of $\mathrm{C}([0,1] ;(X, \tau))$ and the induced quotient space of arcs, obtained by identifying two curves $\gamma_{1}, \gamma_{2} \in \mathrm{C}([0,1] ;(X, \tau))$ if there exist continuous, nondecreasing and surjective maps $\sigma_{1}, \sigma_{2}:[0,1] \rightarrow[0,1]$ such that $\gamma_{1} \circ \sigma_{1}=\gamma_{2} \circ \sigma_{2}$. This provides the natural quotient topology for the space of continuous and $\mathrm{d}$-rectifiable arcs $\mathrm{RA}(X)$, for which the length

$$
\ell(\gamma):=\sup \left\{\sum_{j=1}^{N} \mathrm{~d}\left(\gamma\left(t_{j}\right), \gamma\left(t_{j-1}\right)\right):\left\{t_{j}\right\}_{j=0}^{N} \subset[0,1], \quad t_{0}<t_{1}<\cdots<t_{N}\right\}
$$

is finite. It results a natural metric-topological structure for the space $\mathrm{RA}(X)$, where the distance $\mathrm{d}$ characterizes the length and the integrals, whereas the topology $\tau$ induces the appropriate notion of convergence. This analysis plays a crucial role, since one of the main tools for studying Sobolev spaces involves dynamic plans, i.e. Radon measures on $\mathrm{RA}(X)$. It is also the natural setting to study the properties of length-conformal distances (Section 4).

The second part is devoted to the construction of the Cheeger energy [23,9] (Section 5), the $L^{p}(X, \mathfrak{m})$ relaxation of the energy functional

$$
\int_{X}(\operatorname{lip} f(x))^{p} \mathrm{dm}(x), \quad \operatorname{lip} f(x):=\limsup _{y, z \rightarrow x} \frac{|f(y)-f(x)|}{\mathrm{d}(y, z)},
$$

initially defined on a distinguished unital algebra $\mathscr{A}$ of bounded, $\tau$-continuous and d-Lipschitz functions satisfying the approximation property

$$
\mathrm{d}(x, y)=\sup \left\{f(x)-f(y): f \in \mathscr{A},\left|f\left(x^{\prime}\right)-f\left(y^{\prime}\right)\right| \leq \mathrm{d}\left(x^{\prime}, y^{\prime}\right) \text { for every } x^{\prime}, y^{\prime} \in X\right\}
$$


for every couple of points $x, y \in X$. This gives raise to the Cheeger energy

$$
\mathrm{CE}_{p, \mathscr{A}}(f):=\inf \left\{\int_{X}\left(\operatorname{lip} f_{n}\right)^{p} \mathrm{~d} \mathfrak{m}: f_{n} \in \mathscr{A}, f_{n} \rightarrow f \text { in } L^{p}(X, \mathfrak{m})\right\},
$$

whose proper domain characterizes the strongest Sobolev space (deeply inspired by the Cheeger approach [23])

$$
H^{1, p}(\mathbb{X}, \mathscr{A}):=\left\{f \in L^{p}(X, \mathfrak{m}): \mathrm{CE}_{p, \mathscr{A}}(f)<\infty\right\} .
$$

We will discuss various useful properties of the Cheeger energy, in particular its local representation in terms of the minimal relaxed gradient $|\mathrm{D} f|_{\star, \mathscr{A}}$ as

$$
\mathrm{CE}_{p, \mathscr{A}}(f)=\int_{X}|\mathrm{D} f|_{\star, \mathscr{A}}^{p}(x) \mathrm{d} \mathfrak{m}(x)
$$

the non-smooth first-order calculus properties of $|\mathrm{D} f|_{\star, \mathscr{A}}$, and the invariance properties of $\mathrm{CE}_{p, \mathscr{A}}$ with respect to measure-preserving isometric imbedding of $X$.

A first, non obvious, important result is the independence of $\mathrm{CE}_{p, \mathscr{A}}$ with respect to $\mathscr{A}$, at least when $(X, \tau)$ is compact. It is a consequence of a delicate and powerful approximation method based on the metric Hopf-Lax flow

$$
\mathrm{Q}_{t} f(x):=\inf _{y \in X} f(y)+\frac{1}{q t^{q-1}} \mathrm{~d}^{q}(x, y), \quad t>0,
$$

which we will discuss in great detail in Section 6.

The third part of these notes deals with the basic tools needed for the dual characterization of the Sobolev spaces. First of all the notion of $p$-Modulus [31, 45, 47, 58] (Section 7) of a collection of (nonparametric) rectifiable arcs $\Gamma \subset \mathrm{RA}(X)$,

$$
\operatorname{Mod}_{p}(\Gamma):=\inf \left\{\int_{X} f^{p} \mathrm{dm}: f: X \rightarrow[0, \infty] \text { Borel, } \int_{\gamma} f \geq 1 \text { for every } \gamma \in \Gamma\right\},
$$

which is mainly used to give a precise meaning to negligible sets. It will be put in duality with the class $\mathcal{B}_{q}$ of Radon measures $\boldsymbol{\pi}$ on $\operatorname{RA}(X)$ with finite $q$-barycentric entropy $\operatorname{Bar}_{q}(\boldsymbol{\pi})$ with respect to $\mathfrak{m}$ [2] (Section 8). Every $\boldsymbol{\pi} \in \mathcal{B}_{q}$ induces a measure $\mu_{\boldsymbol{\pi}}=h_{\boldsymbol{\pi}} \mathfrak{m}$ with density $h_{\boldsymbol{\pi}} \in L^{q}(X, \mathfrak{m})$ such that for every bounded Borel function $\zeta: X \rightarrow \mathbb{R}$

$$
\int_{\mathrm{RA}(X)} \int_{\gamma} \zeta \mathrm{d} \boldsymbol{\pi}(\gamma)=\int_{X} \zeta \mathrm{d} \mu_{\boldsymbol{\pi}}=\int_{X} \zeta h_{\boldsymbol{\pi}} \mathrm{d} \mathfrak{m}, \quad \operatorname{Bar}_{q}^{q}(\boldsymbol{\pi}):=\int_{X} h_{\boldsymbol{\pi}}^{q} \mathrm{~d} \mathfrak{m} .
$$

At least when $(X, \mathrm{~d})$ is complete, we will show (Section 9) that the Modulus of a Borel subset $\Gamma \subset$ $\mathrm{RA}(X)$ can be essentially identified with the conjugate of the $q$-barycentric entropy:

$$
\frac{1}{p} \operatorname{Mod}_{p}(\Gamma)=\sup _{\boldsymbol{\pi} \in \mathcal{B}_{q}} \boldsymbol{\pi}(\Gamma)-\frac{1}{q} \operatorname{Bar}_{q}^{q}(\boldsymbol{\pi})
$$

The duality formula shows that a Borel set $\Gamma \subset \operatorname{RA}(X)$ is $\operatorname{Mod}_{p}$-negligible if and only if it is $\pi$ negligible for every dynamic plan $\pi$ with finite $q$-barycentric entropy.

The final part of the notes is devoted to the dual/weak formulation of the Sobolev spaces $W^{1, p}(\mathbb{X})$ and to their relations with the spaces $H^{1, p}(\mathbb{X})$ obtained by the Cheeger construction. The crucial concept 
here is the notion of upper gradient $[45,47,23]$ of a function $f: X \rightarrow \mathbb{R}$ : it is a nonnegative Borel function $g: X \rightarrow[0,+\infty]$ such that

$$
\left|f\left(\gamma_{1}\right)-f\left(\gamma_{0}\right)\right| \leq \int_{\gamma} g
$$

for every rectifiable arc $\gamma \in \mathrm{RA}(X) ; \gamma_{0}$ and $\gamma_{1}$ in (1.11) denote the initial and final points of $\gamma$. As suggested by the theory of Newtonian spaces [58], it is possible to adapt the notion of upper gradient to Sobolev functions by asking that $g \in L^{p}(X, \mathfrak{m})$, by selecting a corresponding notion of "exceptional" or "negligible" sets of rectifiable arcs in $\mathrm{RA}(X)$, and by imposing that the set of curves where (1.11) does not hold is exceptional.

According to the classic approach leading to Newtonian spaces, a subset $\Gamma \subset \mathrm{RA}(X)$ is negligible if $\operatorname{Mod}_{p}(\Gamma)=0$. This important notion, however, is not invariant with respect to modification of $f$ and $g$ in $\mathfrak{m}$-negligible sets. Here we present a different construction, based on the new class $\mathcal{T}_{q}$ of dynamic plans $\pi$ with finite $q$-barycentric entropy and with finite $q$-entropy of the initial and final distribution of points,

$$
\boldsymbol{\pi} \in \mathcal{T}_{q} \quad \Leftrightarrow \quad \operatorname{Bar}_{q}(\boldsymbol{\pi})<\infty, \quad\left(\mathrm{e}_{i}\right)_{\sharp} \boldsymbol{\pi}=h_{i} \mathfrak{m} \quad \text { for some } h_{i} \in L^{q}(X, \mathfrak{m}), \quad i=0,1,
$$

where $\mathrm{e}_{i}(\gamma)=\gamma_{i}$. The last condition requires that there exist functions $h_{i} \in L^{q}(X, \mathfrak{m})$ such that

$$
\int_{\operatorname{RA}(X)} \zeta\left(\gamma_{i}\right) \mathrm{d} \boldsymbol{\pi}(\gamma)=\int_{X} \zeta(x) h_{i} \mathrm{dm} \quad \text { for every bounded Borel function } \zeta: X \rightarrow \mathbb{R} .
$$

A collection $\Gamma \subset \operatorname{RA}(X)$ is $\mathcal{T}_{q}$-negligible if it is $\pi$-negligible for every $\boldsymbol{\pi} \in \mathcal{T}_{q}$. The Sobolev space $W^{1, p}\left(\mathbb{X} . \mathcal{T}_{q}\right)$ precisely contains all the functions $f \in L^{p}(X, \mathfrak{m})$ with a $\mathcal{T}_{q}$-weak upper gradient $g \in L^{p}(X, \mathfrak{m})$, so that

$$
\left|f\left(\gamma_{1}\right)-f\left(\gamma_{0}\right)\right| \leq \int_{\gamma} g \quad \text { for } \mathcal{T}_{q} \text {-a.e. } \operatorname{arc} \gamma \in \mathrm{RA}(X)
$$

Among all the $\mathcal{T}_{q}$-weak upper gradient $g$ of $f$ it is possible to select the minimal one, denoted by $|\mathrm{D} f|_{w, \mathcal{T}_{q}}$, such that $|\mathrm{D} f|_{w, \mathcal{T}_{q}} \leq g$ for every $\mathcal{T}_{q^{\text {-weak }}}$ upper gradient $g$. The norm of $W^{1, p}\left(\mathbb{X}, \mathcal{T}_{q}\right)$ is then given by

$$
\|f\|_{W^{1, p}\left(\mathbb{X}, \mathcal{T}_{q}\right)}^{p}:=\int_{X}\left(|f|^{p}+|\mathrm{D} f|_{w, \mathcal{T}_{q}}^{p}\right) \mathrm{d} \mathfrak{m} .
$$

Differently from the Newtonian weak upper gradient, the notion of $\mathcal{T}_{q}$-weak upper gradient is invariant w.r.t. modifications of $f$ and $g$ in $\mathfrak{m}$-negligible sets; moreover it is possible to prove that functions in $W^{1, p}\left(\mathbb{X}, \mathcal{T}_{q}\right)$ are Sobolev along $\mathcal{T}_{q}$-a.e. arc $\gamma$ with distributional derivative bounded by $g \circ \gamma$. The link with the Newtonian theory appears more clearly by a further properties of functions in $W^{1, p}\left(\mathbb{X}, \mathcal{T}_{q}\right)$, at least when $(X, \mathrm{~d})$ is complete: for every function $f \in W^{1, p}\left(\mathbb{X}, \mathcal{T}_{q}\right)$ it is possible to find a "good representative" $\tilde{f}$ (so that $\{\tilde{f} \neq f\}$ is m-negligible), so that the modified function $\tilde{f}$ is absolutely continuous along $\operatorname{Mod}_{p}$-a.e. rectifiable curve. In this way, the a-priori weaker approach by $\mathcal{T}_{q}$-dynamic plans is equivalent to the Newtonian one and it is possible to identify $W^{1, p}\left(\mathbb{X}, \mathcal{T}_{q}\right)$ with $N^{1, p}(\mathbb{X})$. We will also show in that the approach by nonparametric dynamic plans is equivalent to the definition by parametric $q$-test plans of $[9,8]$.

A further main identification result is stated in Section 11: when $(X, \mathrm{~d})$ is complete, we can show that $W^{1, p}\left(\mathbb{X}, \mathcal{T}_{q}\right)$ coincides with $H^{1, p}(\mathbb{X}, \mathscr{A})$. This fact (originally proved by $[23,58]$ in the case of doubling-Poincaré spaces) can be interpreted as a density result of a compatible algebra of functions 
$\mathscr{A}$ in $W^{1, p}\left(\mathbb{X}, \mathcal{T}_{q}\right)$ and has important consequences, some of them recalled in the last section of the notes. Differently from other recent approaches $[9,8]$ the proof arises from a direct application of the Von Neumann min-max principle and relies on two equivalent characterizations of the dual Cheeger energy $\mathrm{CE}_{p}^{*}(h)$ for functions $h \in L^{q}(X, \mathfrak{m})$

$$
\frac{1}{q} \mathrm{CE}_{p}^{*}(h)=\sup _{h \in H^{1, p}(\mathbb{X})} \int_{X} f h \mathrm{~d} \mathfrak{m}-\frac{1}{p} \mathrm{CE}_{p}(f) \quad h \in L^{q}(X, \mathfrak{m}), \int_{X} h \mathrm{~d} \mathfrak{m}=0 .
$$

When $(X, \tau)$ is compact we can prove that

$$
\mathrm{CE}_{p}^{*}(h)=\sup \left\{\operatorname{Bar}_{q}^{q}(\boldsymbol{\pi}):\left(\mathrm{e}_{0}\right)_{\sharp} \boldsymbol{\pi}=h_{-} \mathfrak{m}, \quad\left(\mathrm{e}_{1}\right)_{\sharp} \boldsymbol{\pi}=h_{+} \mathfrak{m}\right\},
$$

$h_{-}, h_{+}$being the negative and positive parts of $h$, and

$$
\frac{1}{q} \mathrm{CE}_{p}^{*}(h)=\sup \left\{\mathrm{K}_{\mathrm{d}_{g}}\left(h_{-} \mathfrak{m}, h_{+} \mathfrak{m}\right)-\frac{1}{q} \int_{X} g^{q} \mathrm{~d} \mathfrak{m}: g \in \mathrm{C}_{b}(X), \inf _{X} g>0\right\},
$$

where $\mathrm{K}_{\mathbf{d}_{g}}$ is the Kantorovich-Rubinstein distance induced by the cost

$$
\mathrm{d}_{g}\left(x_{0}, x_{1}\right):=\inf \left\{\int_{\gamma} g: \gamma \in \operatorname{RA}(X), \gamma_{0}=x_{0}, \gamma_{1}=x_{1}\right\} .
$$

Thanks to the identification Theorem and the compactification method, we obtain that for a general complete space $(X, \mathrm{~d})$

$$
H^{1, p}(\mathbb{X}, \mathscr{A})=H^{1, p}(\mathbb{X})=W^{1, p}\left(\mathbb{X}, \mathcal{T}_{q}\right)=N^{1, p}(\mathbb{X})
$$

(the last identity holds up to the selection of a good representative) with equality of the corresponding minimal gradients. As a consequence, all the approaches lead to one canonical object and this property does not rely on the validity of doubling properties or Poincaré inequalities for $\mathfrak{m}$.

In the last Section 12 we will show various invariance properties of the Cheeger energy and the metric Sobolev spaces. In particular, when the underlying space $X$ has a linear structure, we show that the metric approach coincides with more classic definitions of Sobolev spaces (e.g. the weighted Sobolev spaces in $\mathbb{R}^{d}[44]$ or the Sobolev spaces associated to a log-concave measure in a BanachHilbert space), obtaining the reflexivity (resp. the Hilbertianity) of $W^{1, p}(\mathbb{X})$ whenever $X$ is a reflexive Banach (resp. Hilbert) space.

A substantial part of these Lecture notes relies on well established theories: our main sources have been $[9,8,12]$ (for the parts concerning the Cheeger energy, the weak upper gradients, and the properties of the Hopf-Lax flow), [17, 46, 2] (for the notion of the $p$-Modulus and the Newtonian spaces), [2] (for the notion of nonparametric dynamic plans in $\mathcal{B}_{q}$, the dual characterization of the $p$-Modulus and the selection of a good representative of a Sobolev function), [3] (for the extended metric-topological structures), [6, 65] (for the results involving the Kantorovich-Rubinstein distances of Optimal Transport), [57] for the theoretic aspects of Radon measures. Further bibliographical notes are added to each Section with more detailed comments. We also refer to the overviews and lecture notes $[15,43,4,36]$.

New contributions concern the role of general compatible algebras of Lipschitz functions and their invariance in the construction of the Cheeger energy, the embedding/compactification tricks, the use of nonparametric dynamic plans for the definition of weak upper gradients, the characterization of the dual Cheeger energy and the proof of the identification theorem $H=W$ by a direct duality argument.

Of course, there are many important aspects that we did not include in these notes: just to name a few of them at the level of the Sobolev construction we quote 
- the Hajłasz's Sobolev spaces [42],

- the theoretical aspects related to the doubling and to the Poincaré inequality assumptions [17, 46],

- the point of view of parametric dynamic plans (i.e. Radon measures on the space of parametric curves with finite $q$-energy) $[9,8]$ (but see the discussion in Section 10.5),

- the properties of the $L^{2}$-gradient flow of the Cheeger energy,

- the original proof of the " $H=W$ " Theorem $[9,8]$ by a dynamic approach based on the identification of the $L^{2}$-gradient flow of the Cheeger energy with the Kantorovich-Wasserstein gradient flow of the Shannon-Rény entropies,

- the approach [26, 4] by derivations and integration by parts,

- the Gigli's nonsmooth differential structures [35, 37, 36] (see also [38, 39]),

- the applications to metric measure spaces satisfying a lower Ricci curvature bounds $[62,63,51$, $11,5,12,30,13]$. 


\subsection{Main notation}

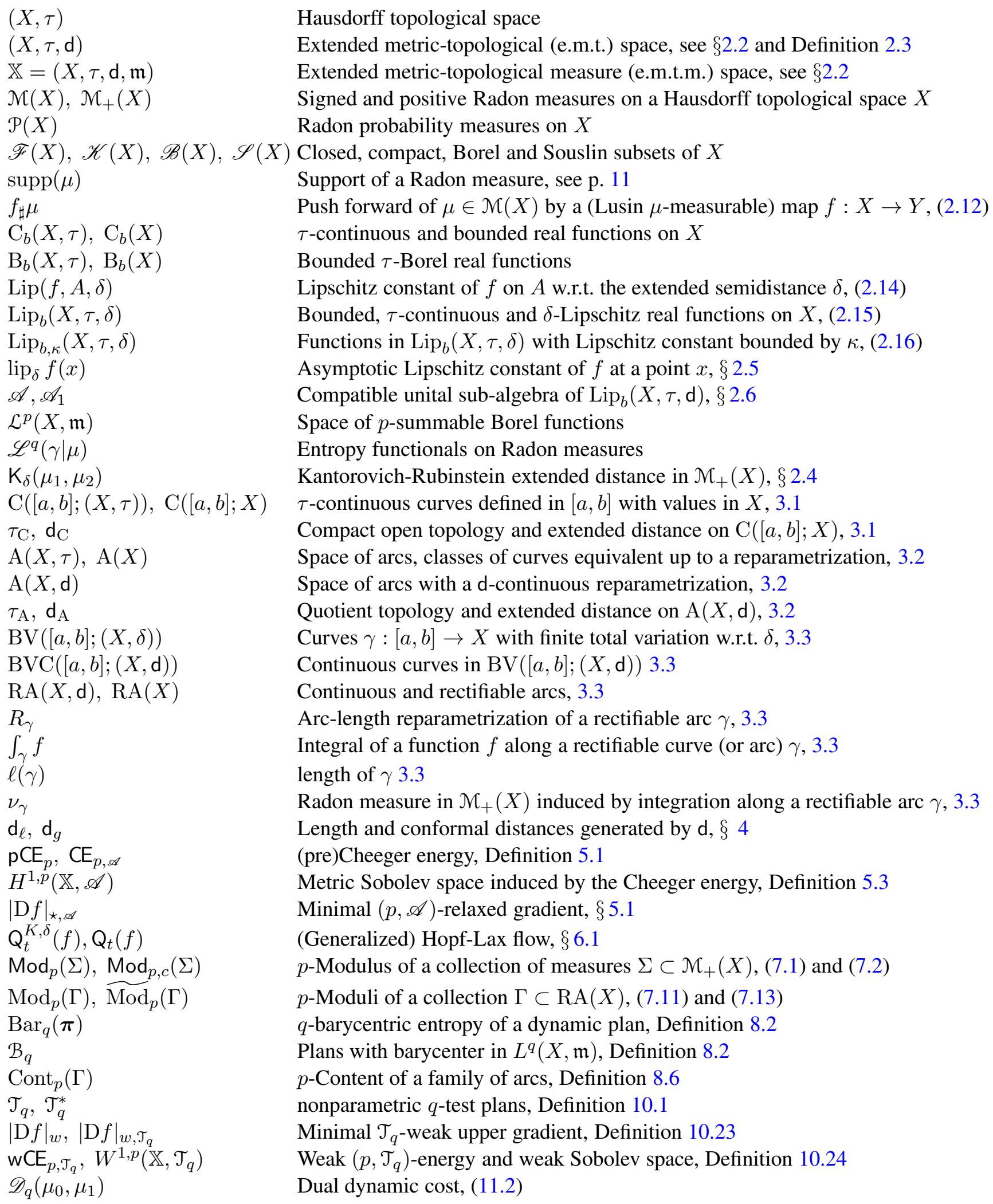

Hausdorff topological space

Extended metric-topological (e.m.t.) space, see $\S 2.2$ and Definition 2.3

Extended metric-topological measure (e.m.t.m.) space, see $\S 2.2$

Signed and positive Radon measures on a Hausdorff topological space $X$ Radon probability measures on $X$

Closed, compact, Borel and Souslin subsets of $X$

Support of a Radon measure, see p. 11

Push forward of $\mu \in \mathcal{M}(X)$ by a (Lusin $\mu$-measurable) map $f: X \rightarrow Y$, (2.12)

$\tau$-continuous and bounded real functions on $X$

Bounded $\tau$-Borel real functions

Lipschitz constant of $f$ on $A$ w.r.t. the extended semidistance $\delta,(2.14)$

Bounded, $\tau$-continuous and $\delta$-Lipschitz real functions on $X,(2.15)$

Functions in $\operatorname{Lip}_{b}(X, \tau, \delta)$ with Lipschitz constant bounded by $\kappa,(2.16)$

Asymptotic Lipschitz constant of $f$ at a point $x, \S 2.5$

Compatible unital sub-algebra of $\operatorname{Lip}_{b}(X, \tau, \mathrm{d}), \S 2.6$

Space of $p$-summable Borel functions

Entropy functionals on Radon measures

Kantorovich-Rubinstein extended distance in $\mathcal{M}_{+}(X), \S 2.4$

$\tau$-continuous curves defined in $[a, b]$ with values in $X, 3.1$

Compact open topology and extended distance on $\mathrm{C}([a, b] ; X), 3.1$

Space of arcs, classes of curves equivalent up to a reparametrization, 3.2

Space of arcs with a d-continuous reparametrization, 3.2

Quotient topology and extended distance on $\mathrm{A}(X, \mathrm{~d}), 3.2$

Curves $\gamma:[a, b] \rightarrow X$ with finite total variation w.r.t. $\delta, 3.3$

Continuous curves in $\mathrm{BV}([a, b] ;(X, \mathrm{~d})) 3.3$

Continuous and rectifiable arcs, 3.3

Arc-length reparametrization of a rectifiable arc $\gamma, 3.3$

Integral of a function $f$ along a rectifiable curve (or arc) $\gamma, 3.3$

length of $\gamma 3.3$

Radon measure in $\mathcal{M}_{+}(X)$ induced by integration along a rectifiable arc $\gamma, 3.3$

Length and conformal distances generated by $d, \S 4$

(pre)Cheeger energy, Definition 5.1

Metric Sobolev space induced by the Cheeger energy, Definition 5.3

Minimal $(p, \mathscr{A})$-relaxed gradient, $\S 5.1$

(Generalized) Hopf-Lax flow, $\S 6.1$

$p$-Modulus of a collection of measures $\Sigma \subset \mathcal{M}_{+}(X),(7.1)$ and (7.2)

$p$-Moduli of a collection $\Gamma \subset \operatorname{RA}(X),(7.11)$ and (7.13)

$q$-barycentric entropy of a dynamic plan, Definition 8.2

Plans with barycenter in $L^{q}(X, \mathfrak{m})$, Definition 8.2

$p$-Content of a family of arcs, Definition 8.6

nonparametric $q$-test plans, Definition 10.1

Minimal $\mathcal{T}_{q}$-weak upper gradient, Definition 10.23

Weak $\left(p, \mathcal{T}_{q}\right)$-energy and weak Sobolev space, Definition 10.24

Dual dynamic cost, (11.2) 


\section{Part I}

\section{Topological and Metric-Measure structures}

\section{Metric-measure structures}

In this section we will recall the main notion and facts we will use in the sequel. Our main ingredients are

- a Hausdorff topological space $(X, \tau)$,

- an extended distance $\mathrm{d}: X \times X \rightarrow[0, \infty]$,

- a finite Radon measure $\mathfrak{m}$ on $(X, \tau)$,

- an algebra $\mathcal{A}$ of $\tau$-continuous, d-Lipschitz, bounded real functions defined in $X$.

All these objects will satisfy suitable compatibility conditions, which we are going to explain. We will call the system

$$
\mathbb{X}=(X, \tau, \mathrm{d}, \mathfrak{m}) \text {, an extended metric-topological measure (e.m.t.m.) space. }
$$

The choice of $\mathcal{A}$ will play a role in the construction of the Cheeger energy.

Let us first consider the topological and measurable side of this structure.

\subsection{Topological and measure theoretic notions}

Let $(X, \tau)$ be a Hausdorff topological space. We will denote by $\mathrm{C}_{b}(X, \tau)$ (resp. $\left.\mathrm{B}_{b}(X, \tau)\right)$ the space of $\tau$-continuous (resp. Borel) and bounded real functions defined on $X . \mathscr{B}(X, \tau)$ is the collection of the Borel subsets of $X$. For every $x \in X, \mathscr{U}_{x}$ will denote the system of neighborhoods of $x$. We will often omit the explicit indication of the topology $\tau$, when it will be clear from the context.

We will always deal with a completely regular topology, i.e.

$$
\begin{aligned}
& \text { for any closed set } F \subset X \text { and any } x_{0} \in X \backslash F \\
& \text { there exists } f \in \mathrm{C}_{b}(X, \tau) \text { with } f\left(x_{0}\right)>0 \text { and } f \equiv 0 \text { on } F \text {. }
\end{aligned}
$$

We can always assume that $f$ takes values in $[0,1]$ and $f\left(x_{0}\right)=1$. An immediate consequence of (2.2) is that for every open subset $G \subset X$ its characteristic function $\chi_{G}$ can be represented as

$$
\chi_{G}(x)=\sup \left\{\varphi(x): \varphi \in \mathrm{C}_{b}(X, \tau), 0 \leq \varphi \leq \chi_{G}\right\},
$$

and the same representation holds for every nonnegative lower semicontinuous (1.s.c.) $f: X \rightarrow$ $[0,+\infty]$ :

$$
f(x)=\sup \left\{\varphi(x): \varphi \in \mathrm{C}_{b}(X, \tau), 0 \leq \varphi \leq f\right\}, \quad f: X \rightarrow[0,+\infty] \text { 1.s.c. }
$$

Definition 2.1 (Radon measures [57, Chap. I, Sect. 2]). A finite Radon measure $\mu: \mathscr{B}(X, \tau) \rightarrow$ $[0,+\infty)$ is a Borel nonnegative $\sigma$-additive finite measure satisfying the following inner regularity property:

$$
\forall B \in \mathscr{B}(X, \tau): \quad \mu(B)=\sup \{\mu(K): K \subset B, K \text { compact }\} .
$$


A finite Radon measure $\mu$ is also outer regular:

$$
\forall B \in \mathscr{B}(X, \tau): \quad \mu(B)=\inf \{\mu(O): O \subset X, O \text { open }\} .
$$

We will denote by $\mathcal{M}_{+}(X)$ (resp. $\mathcal{P}(X)$ ) the collection of all finite (resp. Probability) Radon measures on $X$. By the very definition of Radon topological space [57, Ch. II, Sect. 3], every Borel measure in a Radon space is Radon: such class of spaces includes locally compact spaces with a countable base of open sets, Polish, Lusin and Souslin spaces. In particular the notation of $\mathcal{P}(X)$ is consistent with the standard one adopted e.g. in [10, 7, 64], where Polish or second countable locally compact spaces are considered.

(2.5) implies in particular that a Radon measure is tight:

$$
\forall \varepsilon>0 \quad \exists K_{\varepsilon} \subset X \quad \text { compact such that } \mu\left(X \backslash K_{\varepsilon}\right) \leq \varepsilon .
$$

We can also define in the usual way the support $\operatorname{supp} \mu$ of a Radon measure as the set of points $x \in X$ such that every neighborhood $U \in \mathscr{U}_{x}$ has strictly positive measure $\mu(U)>0$. Thanks to (2.5), one can verify that $\mu(X \backslash \operatorname{supp}(\mu))=0[57$, p. 60].

Radon measures have stronger additivity and continuity properties in connection with open sets and lower semicontinuous functions; in particular we shall use this version of the monotone convergence theorem (see [19, Lemma 7.2.6])

$$
\lim _{i \in I} \int f_{i} \mathrm{~d} \mu=\int \lim _{i \in I} f_{i} \mathrm{~d} \mu
$$

valid for Radon measures $\mu$ and for nondecreasing nets $i \mapsto f_{i}, i \in I$, of $\tau$-lower semicontinuous and equibounded functions $f_{i}: X \rightarrow[0, \infty]$. Here $I$ is a directed set with a partial order $\preceq$ satisfying $i \preceq j \Rightarrow f_{i} \leq f_{j}$, see the Appendix A.1.

The weak (or narrow) topology $\tau_{\mathcal{M}_{+}}$on $\mathcal{M}_{+}(X)$ can be defined as the coarsest topology for which all maps

$$
\mu \mapsto \int h \mathrm{~d} \mu \quad \text { from } \mathcal{M}_{+}(X) \text { into } \mathbb{R}
$$

are continuous as $h: X \rightarrow \mathbb{R}$ varies in $\mathrm{C}_{b}(X, \tau)$ [57, p. 370, 371].

Prokhorov Theorem provides a sufficient condition for compactness w.r.t. the weak topology: [57, Theorem 3, p. 379].

Theorem 2.2 (Prokhorov). Let $(X, \tau)$ be a completely regular Hausdorff topological space. Assume that a collection $\mathcal{K} \subset \mathcal{M}_{+}(X)$ is uniformly bounded and equi-tight, i.e.

$$
\begin{gathered}
\qquad \sup _{\mu \in \mathcal{K}} \mu(X)<\infty, \\
\text { for every } \varepsilon>0 \text { there exists a compact set } K_{\varepsilon} \subset X \text { such that } \sup _{\mu \in \mathcal{K}} \mu\left(X \backslash K_{\varepsilon}\right) \leq \varepsilon .
\end{gathered}
$$

Then $\mathcal{K}$ has limit points in the class $\mathcal{M}_{+}(X)$ w.r.t. the weak topology.

Recall that a set $A \subset X$ is $\mathfrak{m}$-measurable if there exist Borel sets $B_{1}, B_{2} \in \mathscr{B}(X, \tau)$ such that $B_{1} \subset A \subset B_{2}$ and $\mathfrak{m}\left(B_{2} \backslash B_{1}\right)=0$. m-measurable sets form a $\sigma$-algebra $\mathscr{B}_{\mathfrak{m}}(X)$. A set is called universally (Radon) measurable if it is $\mu$-measurable for every Radon measure $\mu \in \mathcal{M}_{+}(X)$.

Let $\left(Y, \tau_{Y}\right)$ be a Hausdorff topological space. A map $f: X \rightarrow Y$ is Borel (resp. Borel $\mathfrak{m}$ measurable) if for every $B \in \mathscr{B}(Y) f^{-1}(B) \in \mathscr{B}(X)$ (resp. $f^{-1}(B)$ is $\mathfrak{m}$-measurable). $f$ is Lusin 
m-measurable if for every $\varepsilon>0$ there exists a compact set $K_{\varepsilon} \subset X$ such that $\mathfrak{m}\left(X \backslash K_{\varepsilon}\right) \leq \varepsilon$ and the restriction of $f$ to $K_{\varepsilon}$ is continuous. A map $f: X \rightarrow Y$ is called universally measurable if it is Lusin $\mu$-measurable for every Radon measure $\mu \in \mathcal{M}_{+}(X)$.

Every Lusin $\mathfrak{m}$-measurable map is also Borel $\mathfrak{m}$-measurable; the converse is true if, e.g., the topology $\tau_{Y}$ is metrizable and separable [57, Chap. I Section 1.5, Theorem 5]. Whenever $f$ is Lusin $\mathfrak{m}$-measurable, its push-forward

$$
f_{\sharp} \mathfrak{m} \in \mathcal{M}_{+}(Y), \quad f_{\sharp} \mathfrak{m}(B):=\mathfrak{m}\left(f^{-1}(B)\right) \quad \text { for every Borel subset } B \subset \mathscr{B}(Y)
$$

induces a Radon measure in $Y$.

Given a power $p \in(1, \infty)$ and a Radon measure $\mathfrak{m}$ in $(X, \tau)$ we will denote by $L^{p}(X, \mathfrak{m})$ the usual Lebesgue space of class of $p$-summable $\mathfrak{m}$-measurable functions defined up to $\mathfrak{m}$-negligible sets. We will also set

$$
\mathcal{L}_{+}^{p}(X, \mathfrak{m}):=\left\{f: X \rightarrow[0, \infty]: f \text { is Borel, } \int_{X} f^{p} \mathrm{~d} \mathfrak{m}<\infty\right\} ;
$$

this space is not quotiented under any equivalence relation. We will keep using the notation

$$
\|f\|_{p}=\|f\|_{L^{p}(X, \mathfrak{m})}:=\left(\int_{X}|f|^{p} \mathrm{~d} \mathfrak{m}\right)^{1 / p}
$$

as a seminorm on $\mathcal{L}_{+}^{p}(X, \mathfrak{m})$ and a norm in $L^{p}(X, \mathfrak{m})$.

\subsection{Extended metric-topological (measure) spaces}

Let $(X, \tau)$ be a Hausdorff topological space.

An extended semidistance is a symmetric map $\delta: X \times X \rightarrow[0, \infty]$ satisfying the triangle inequality; $\delta$ is an extended distance if it also satisfies the property $\delta(x, y)=0$ iff $x=y$ in $X$ : in this case, we call $(X, \delta)$ an extended metric space. We will omit the adjective "extended" if $\delta$ takes real values.

Whenever $f: X \rightarrow \mathbb{R}$ is a given function, $A \subset X$, and $\delta$ is an extended semidistance on $X$, we set

$$
\operatorname{Lip}(f, A, \delta):=\inf \{L \in[0, \infty]:|f(y)-f(z)| \leq L \delta(y, z) \quad \text { for every } y, z \in A\} .
$$

We adopt the convention to omit the set $A$ when $A=X$. We consider the class of $\tau$-continuous and $\delta$-Lipschitz functions

$$
\operatorname{Lip}_{b}(X, \tau, \delta):=\left\{f \in \mathrm{C}_{b}(X, \tau): \operatorname{Lip}(f, \delta)<\infty\right\},
$$

and for every $\kappa>0$ we will also consider the subsets

$$
\operatorname{Lip}_{b, \kappa}(X, \tau, \delta):=\left\{f \in \mathrm{C}_{b}(X, \tau): \operatorname{Lip}(f, \delta) \leq \kappa\right\} .
$$

A particular role will be played by $\operatorname{Lip}_{b, 1}(X, \tau, \delta)$. We will sometimes omit to indicate the explicit dependence on $\tau$ and $\delta$ whenever it will be clear from the context. It is easy to check that $\operatorname{Lip}_{b}(X, \tau, \delta)$ is a real and commutative sub-algebras of $\mathrm{C}_{b}(X, \tau)$ with unit.

According to [3, Definition 4.1], an extended metric-topological space (e.m.t. space) $(X, \tau, \mathrm{d})$ is characterized by a Hausdorff topology $\tau$ and an extended distance $d$ satisfying a suitable compatibility condition. 
Definition 2.3 (Extended metric-topological spaces). Let $(X, \mathrm{~d})$ be an extended metric space, let $\tau$ be a Hausdorff topology in $X$. We say that $(X, \tau, \mathrm{d})$ is an extended metric-topological (e.m.t.) space if:

(X1) the topology $\tau$ is generated by the family of functions $\operatorname{Lip}_{b}(X, \tau, \mathrm{d})$ (see the Appendix A.2);

(X2) the distance $\mathrm{d}$ can be recovered by the functions in $\operatorname{Lip}_{b, 1}(X, \tau, \mathrm{d})$ through the formula

$$
\mathrm{d}(x, y)=\sup _{f \in \operatorname{Lip}_{b, 1}(X, \tau, \mathrm{d})}|f(x)-f(y)| \quad \text { for every } x, y \in X
$$

We will say that $(X, \tau, \mathrm{d})$ is complete if $\mathrm{d}$-Cauchy sequences are $\mathrm{d}$-convergent. All the other topological properties (as compactness, separability, metrizability, Borel, Polish-Lusin-Souslin, etc) usually refers to $(X, \tau)$.

The previous assumptions guarantee that $(X, \tau)$ is completely regular, according to (2.2) (see the Appendix A.2). As in (2.1), when an e.m.t. space $(X, \tau, \mathrm{d})$ is provided by a positive Radon measure $\mathfrak{m} \in \mathcal{M}_{+}(X, \tau)$ we will call the system $\mathbb{X}=(X, \tau, \mathrm{d}, \mathfrak{m})$ an extended metric-topological measure (e.m.t.m.) space.

Definition 2.3 yields two important properties linking $\mathrm{d}$ and $\tau$ : first of all

$$
\mathrm{d} \text { is } \tau \times \tau \text {-lower semicontinuous in } X \times X \text {, }
$$

since it is the supremum of a family of continuo us maps by (2.17). On the other hand, every $\mathrm{d}$ converging net $\left(x_{j}\right)_{j \in J}$ indexed by a directed set $J$ is also $\tau$-convergent:

$$
\lim _{j \in J} \mathrm{~d}\left(x_{j}, x\right)=0 \Rightarrow \lim _{j \in J} x_{j}=x \quad \text { w.r.t. } \tau .
$$

It is sufficient to observe that $\tau$ is the initial topology generated by $\operatorname{Lip}_{b}(X, \tau, \mathrm{d})$ so that a net $\left(x_{j}\right)$ is convergent to a point $x$ if and only if

$$
\lim _{j \in J} f\left(x_{j}\right)=f(x) \quad \text { for every } f \in \operatorname{Lip}_{b}(X, \tau, \mathrm{d}) .
$$

A basis of neighborhoods for the $\tau$-topology at a point $x \in X$ is given by the sets of the form

$$
U_{F, \varepsilon}(x):=\left\{y \in X: \sup _{f \in F}|f(y)-f(x)|<\varepsilon\right\} \quad F \subset \operatorname{Lip}_{b, 1}(X, \tau, \mathrm{d}) \text { finite, } \varepsilon>0 .
$$

Definition 2.3 is in fact equivalent to other seemingly stronger assumptions, as we discuss in the following Lemma.

Lemma 2.4 (Monotone approximations of the distance). Let $(X, \tau, \mathrm{d})$ be an e.m.t. space, let us denote by $\Lambda$ the collection of all the finite subsets in $\operatorname{Lip}_{b, 1}(X, \tau, \mathrm{d})$, a directed set ordered by inclusion, and let us define

$$
\mathrm{d}_{\lambda}(x, y):=\sup _{f \in \lambda}|f(x)-f(y)|, \quad \lambda \in \Lambda, x, y \in X .
$$

The family $\left(\mathrm{d}_{\lambda}\right)_{\lambda \in \Lambda}$ is a monotone collection of $\tau$ continuous and bounded semidistances on $X$ generating the $\tau$-topology and the extended distance $\mathrm{d}$, in the sense that for every net $\left(x_{j}\right)_{j \in J}$ in $X$

$$
x_{j} \stackrel{\tau}{\rightarrow} x \quad \Leftrightarrow \quad \lim _{j \in J} \mathrm{~d}_{\lambda}\left(x_{j}, x\right)=0 \quad \text { for every } \lambda \in \Lambda,
$$


and

$$
\mathrm{d}(x, y)=\sup _{\lambda \in \Lambda} \mathrm{d}_{\lambda}(x, y)=\lim _{\lambda \in \Lambda} \mathrm{d}_{\lambda}(x, y) \quad \text { for every } x, y \in X
$$

Conversely, suppose that $\left(\mathrm{d}_{i}\right)_{i \in I}$ is a directed family of real functions on $X \times X$ satisfying

$$
\begin{gathered}
\mathrm{d}_{i}: X \times X \rightarrow[0,+\infty) \quad \text { is a bounded and continuous semidistance for every } i \in I, \\
i \preceq j \Rightarrow \mathrm{d}_{i} \leq \mathrm{d}_{j}, \\
x_{j} \stackrel{\tau}{\rightarrow} x \Leftrightarrow \quad \lim _{j \in J} \mathrm{~d}_{i}\left(x_{j}, x\right)=0 \quad \text { for every } i \in I, \\
\mathrm{~d}(x, y)=\sup _{i \in I} \mathrm{~d}_{i}(x, y)=\lim _{i \in I} \mathrm{~d}_{i}(x, y) \quad \text { for every } x, y \in X,
\end{gathered}
$$

then $(X, \tau, \mathrm{d})$ is an extended metric-topological space.

Proof. (2.23a) and (2.23b) are immediate consequence of the Definition (2.3). In order to prove the second statement, we simply observe that the collection of functions $\mathcal{F}:=\left\{\mathrm{d}_{i}(y, \cdot): i \in I, y \in X\right\}$ is included in $\operatorname{Lip}_{b, 1}(X, \tau, \mathrm{d})$ and generates the topology $\tau$ thanks to $(2.24 \mathrm{c})$. A fortiori, $\operatorname{Lip}_{b, 1}(X, \tau, \mathrm{d})$ satisfies conditions (X1) and (X2) of Definition 2.3.

We will often use the following simple and useful property involving a directed family of semidistances $\left(\mathrm{d}_{i}\right)_{i \in I}$ satisfying $(2.24 \mathrm{a}, \mathrm{b}, \mathrm{c}, \mathrm{d})$ ): whenever $\mathfrak{i}: J \rightarrow I$ is a subnet and $x_{j}, y_{j}, j \in J$, are $\tau$-converging to $x, y$ respectively, we have

$$
\liminf _{j \in J} \mathrm{~d}_{\mathfrak{i}(j)}\left(x_{j}, y_{j}\right) \geq \mathrm{d}(x, y) .
$$

It follows easily by the continuity of $\mathrm{d}_{i}$ and (2.24b), since for every $i \in I$

$$
\liminf _{j \in J} \mathrm{~d}_{\mathfrak{i}(j)}\left(x_{j}, y_{j}\right) \geq \liminf _{j \in J} \mathrm{~d}_{i}\left(x_{j}, y_{j}\right) \geq \mathrm{d}_{i}(x, y) ;
$$

(2.25) then follows by taking the supremum w.r.t. $i \in I$.

Remark 2.5. Notice that if $K$ is a Souslin subset of $X$ (in particular $K=X$ if $(X, \tau)$ is Souslin) then $K \times K$ is Souslin as well, so that by Lemma A.4(b) there exists a countable collection $\mathcal{F}=$ $\left(f_{n}\right)_{n \in \mathbb{N}} \subset \operatorname{Lip}_{b, 1}(X, \tau, \mathrm{d})$ such that

$$
\mathrm{d}(x, y)=\sup _{n \in \mathbb{N}}\left|f_{n}(x)-f_{n}(y)\right| \quad \text { for every } x, y \in K .
$$

If $\tau^{\prime}$ is the initial topology generated by $\mathcal{F},\left(K, \tau^{\prime}\right.$, d $)$ is an e.m.t. space whose topology $\tau^{\prime}$ is coarser than $\tau . \tau^{\prime}$ is also metrizable and separable: it is sufficient to choose an increasing 1-Lipschitz homeomorphism $\vartheta: \mathbb{R} \rightarrow] 0,1 / 12\left[\right.$ and setting $f_{n}^{\prime}:=\vartheta \circ f_{n}$; the family $\left(f_{n}^{\prime}\right)_{n \in \mathbb{N}}$ induces the same topology $\tau^{\prime}$, it separates the points of $K$, and the distance

$$
\mathrm{d}^{\prime}(x, y):=\sum_{n=1}^{\infty} 2^{-n}\left|f_{n}^{\prime}(x)-f_{n}^{\prime}(y)\right|
$$

is a bounded $\tau$-continuous semidistance dominated by $\mathrm{d}$ whose restriction to $K \times K$ is a distance inducing the topology $\tau^{\prime}$. If $K$ is also compact, than $\tau$ coincides with the topology induced by $\mathrm{d}^{\prime}$. 
Let us recap a useful property discussed in the previous Remark.

Definition 2.6 (Auxiliary topologies). Let $(X, \tau, \mathrm{d})$ be an e.m.t. space. We say that $\tau^{\prime}$ is an auxiliary topology if there exist a countable collection $\mathcal{F}=\left(f_{n}\right)_{n \in \mathbb{N}} \subset \operatorname{Lip}_{b}(X, \tau, \mathrm{d})$ such that $\tau^{\prime}$ is generated by $\mathcal{F}$ and

$$
\mathrm{d}(x, y)=\sup _{n \in \mathbb{N}}\left|f_{n}(x)-f_{n}(y)\right|
$$

Equivalently

(A1) $\tau^{\prime}$ is coarser than $\tau$,

(A2) $\tau^{\prime}$ is separable and metrizable by a bounded $\tau$-continuous distance $\mathrm{d}^{\prime} \leq \mathrm{d}$,

(A3) there exists a sequence $f_{n} \in \operatorname{Lip}_{b}\left(X, \tau^{\prime}, \mathrm{d}\right)$ such that (2.28) holds.

In particular, $\left(X, \tau^{\prime}, \mathrm{d}\right)$ is an e.m.t. space.

If $\tau^{\prime}$ is generated by a countable collection $\mathcal{F} \subset \operatorname{Lip}_{b}(X, \tau, \mathrm{d})$ satisfying (2.28) then properties $(\mathrm{A} 1,2,3)$ obviously hold by the discussion of Remark 2.5 . Conversely, if $\tau^{\prime}$ satisfies $(\mathrm{A} 1,2,3)$ then one can consider the countable collection $\mathcal{F}$ resulting by the union of $\left(f_{n}\right)_{n \in \mathbb{N}}$ given in (A3) and the set $\left\{\mathrm{d}^{\prime}\left(x_{n}, \cdot\right)\right\}_{n \in \mathbb{N}}$ where $\left(x_{n}\right)_{n \in \mathbb{N}}$ is a $\tau^{\prime}$ dense subset of $X$ and $\mathrm{d}^{\prime}$ is given by (A2). It is clear that $\tau^{\prime}$ is the initial topology of $\mathcal{F}$ and (2.28) holds.

By setting

$$
\mathrm{d}_{n}(x, y):=\sup _{1 \leq k \leq n}\left|f_{k}(x)-f_{k}(y)\right|
$$

one can easily see that (A3) is in fact equivalent to

(A3') There exists an increasing sequence of $\tau^{\prime}$ continuous and bounded (semi)distances $\left(\mathrm{d}_{n}\right)_{n \in \mathbb{N}}$ such that

$$
\mathrm{d}(x, y)=\sup _{n \in \mathbb{N}} \mathrm{d}_{n}(x, y)=\lim _{n \rightarrow \infty} \mathrm{d}_{n}(x, y) \quad \text { for every } x, y \in X .
$$

It is also possible to assume $\mathrm{d}_{n} \geq \mathrm{d}^{\prime}$ for every $n \in \mathbb{N}$.

As a consequence of Remark 2.5 we have:

Corollary 2.7 (Auxiliary topologies for Souslin e.m.t. spaces). If $(X, \tau, \mathrm{d})$ is a Souslin e.m.t. space (i.e. $(X, \tau)$ is Souslin) then it admits an auxiliary topology $\tau^{\prime}$ according to Definition 2.6.

Notice that if $\tau^{\prime}$ is an auxiliary topology of a Souslin e.m.t. space $(X, \tau, \mathrm{d}),\left(X, \tau^{\prime}\right)$ is Souslin as well. If $\mathfrak{m}$ is a Radon measure in $(X, \tau)$ then it is Radon also w.r.t. $\tau^{\prime}$. An important consequence of the existence of an auxiliary topology is the following fact:

Lemma 2.8. If $(X, \tau, \mathrm{d})$ admits an auxiliary topology $\tau^{\prime}$ then every $\tau$-compact set $K \subset X$ is a Polish space (with the relative topology).

Proof. It is sufficient to note that $\tau$ and $\tau^{\prime}$ induces the same topology on $K$ and that $\tau^{\prime}$ is metrizable and separable. 


\subsection{Examples}

Example 2.9 (Complete and separable metric spaces). The most important and common example is provided by a complete and separable metric space $(X, \mathrm{~d})$; in this case, the canonical choice of $\tau$ is the (Polish) topology induced by $d$. Any positive and finite Borel measure $\mathfrak{m}$ on $(X, \mathrm{~d})$ is a Radon measure so that $(X, \mathrm{~d}, \mathfrak{m})$ is a Polish metric measure space. This case cover the Euclidean spaces $\mathbb{R}^{d}$, the complete Riemannian or Finsler manifolds, the separable Banach spaces and their closed subsets.

In some situation, however, when $(X, \mathrm{~d})$ is not separable or $\mathrm{d}$ takes the value $+\infty$, it could be useful to distinguish between the topological and the metric aspects. This will particularly important when a measure will be involved, since the Radon property with respect to a coarser topology is less restrictive.

Example 2.10 (Dual of a Banach space). A typical example is provided by the dual $X=B^{\prime}$ of a separable Banach space: in this case the distance $d$ is induced by the dual norm $\|\cdot\|_{B^{\prime}}$ of $B^{\prime}$ (which may not be separable) and the topology $\tau$ is the weak* topology of $B^{\prime}$, which is Lusin [57, Corollary 1, p. 115]. All the functions of the form $f(x ; y, v, r):=\mathrm{t}(\langle x-y, v\rangle)$ where $y \in B^{\prime}, \mathrm{t}: \mathbb{R} \rightarrow \mathbb{R}$ is a bounded 1-Lipschitz map and $v \in B$ with $\|v\|_{B} \leq 1$ clearly belong to $\operatorname{Lip}_{b}(X, \tau, \mathrm{d})$ and are sufficient to recover the distance $d$ since

$$
\mathrm{d}(x, y)=\|x-y\|_{B^{\prime}}=\sup _{\|v\|_{B} \leq 1}\langle x-y, v\rangle=\sup _{\|v\| \leq 1} f(x ; y, v, \mathrm{t}), \quad \mathrm{t}(r):=0 \vee r \wedge(2\|x-y\|) .
$$

A slight modification of the previous setting leads to a somehow universal model: we will see in $\S 2.7$ that every e.m.t. space can be isometrically and continuously embedded in such a framework and every metric Sobolev space has an isomorphic representation in this setting (see Corollary 12.16).

Example 2.11. Let $X$ be weakly* compact subset of a dual Banach space $B^{\prime}$ endowed with the weak* topology $\tau$ and a Radon measure $\mathfrak{m}$. We select a strongly closed and symmetric convex set $L \subset B$ containing 0 and separating the points of $B^{\prime}$ and we set

$$
\theta(z):=\sup _{f \in L}\langle z, f\rangle, \quad \mathrm{d}(x, y):=\theta(x-y) .
$$

It is immediate to check that $(X, \tau, \mathrm{d})$ is an e.m.t. space. Notice that $\theta$ is 1 -homogeneous and convex, therefore it is an "extended" norm (possibly assuming the value $+\infty$ ), so that $\mathrm{d}$ is translation invariant. The previous Example 2.10 correspond to the case when $L$ is the unit ball of $B$.

Example 2.12 (Abstract Wiener spaces). Let $\left(X,\|\cdot\|_{X}\right)$ be a separable Banach space endowed with a Radon measure $\mathfrak{m}$ and let $\left(W,|\cdot|_{W}\right)$ be a reflexive Banach space (in particular an Hilbert space) densely and continuously included in $X$, so that there exists a constant $C>0$ such that

$$
\|h\|_{X} \leq C|h|_{W} \quad \text { for every } h \in W .
$$

We call $\tau$ the Polish topology of $X$ induced by the Banach norm and for every $x, y, z \in X$ we set

$$
\phi(z):=\left\{\begin{array}{ll}
|z|_{W} & \text { if } z \in W, \\
+\infty & \text { otherwise, }
\end{array} \quad \mathrm{d}(x, y):=\phi(x-y)= \begin{cases}|x-y|_{W} & \text { if } x-y \in W \\
+\infty & \text { otherwise }\end{cases}\right.
$$

The functional $z \mapsto \phi(z)$ is 1-homogenous, convex and lower semicontinuous in $X$ (thanks to the reflexivity of $W$ ) so that setting

$$
L:=\left\{f \in X^{\prime}:\langle f, z\rangle \leq|z|_{B} \text { for every } z \in B\right\},
$$


Fenchel duality yields

$$
\phi(z)=\sup _{f \in L}\langle f, z\rangle, \quad \mathrm{d}(x, y)=\sup _{f \in L}\langle f, x-y\rangle ;
$$

the same truncation trick of Example 2.10 shows that (2.17) is satisfied. On the other hand, the distance functions $x \mapsto\|x-z\|_{X}, z \in X$, induced by the norm in $X$ belong to $\operatorname{Lip}_{b}(X, \tau, \mathrm{d})$ so that the first condition of Definition 2.3 is satisfied as well. This setting covers the important case of an abstract Wiener space, when $\mathfrak{m}$ is a Gaussian measure in $X$ and $W$ is the Cameron-Martin space, see e.g. [18].

Example 2.13. Let $X:=\mathbb{R}^{d}$ and let $h: X \times \mathbb{R}^{d} \rightarrow[0,+\infty]$ be a lower semicontinuous function such that for every $x \in X$

$h(x, \cdot) \quad$ is 1-homogeneous and convex, $h(x, v)>h(x, 0)=0 \quad$ for every $x \in X, v \in \mathbb{R}^{d} \backslash\{0\}$.

We can define the extended "Finsler" distance

$$
\mathrm{d}\left(x_{0}, x_{1}\right):=\inf \left\{\int_{0}^{1} h\left(\mathrm{x}(t), \mathrm{x}^{\prime}(t)\right) \mathrm{d} t: \mathrm{x} \in \operatorname{Lip}\left([0,1], \mathbb{R}^{d}\right), \quad \mathrm{x}(i)=x_{i}, i=0,1\right\}
$$

with the convention that $\mathrm{d}\left(x_{0}, x_{1}\right)=+\infty$ if there is no Lipschitz curve connecting $x_{0}$ to $x_{1}$ with a finite cost. When there exist constants $C_{0}, C_{1}>0$ such that

$$
C_{0}|v| \leq h(x, v) \leq C_{1}|v| \quad \text { for every } x, v \in \mathbb{R}^{d},
$$

$\mathrm{d}$ is the "Finsler" distance induced by the family of norms $(h(x, \cdot))_{x \in \mathbb{R}^{d}}$, inducing the usual topology of $\mathbb{R}^{d}$.

When

$$
h(x, v)= \begin{cases}|v| & \text { if } x \in X_{0}, \\ +\infty & \text { if } x \in \mathbb{R}^{d} \backslash X_{0}, v \neq 0, \quad X_{0} \quad \text { is a closed subset of } \mathbb{R}^{d},\end{cases}
$$

then $\mathrm{d}$ is the "geodesic extended distance" induced by the Euclidean tensor on $X_{0}$. When $h$ is expressed in terms of a smooth family of bounded vector fields $\left(X_{j}\right)_{j=1}^{J}, X_{j}: \mathbb{R}^{d} \rightarrow \mathbb{R}^{d}$, by the formula

$$
h^{2}(x, v):=\inf \left\{\sum_{j=1}^{J} u_{j}^{2}: \sum_{j=1}^{J} u_{j} X_{j}(x)=v\right\}
$$

we obtain the Carnot-Caratheodory distance induced by the vector fields $X_{j}$. In all these cases, we can approximate $h$ by its Yosida regularization:

$$
h_{\varepsilon}^{2}(x, v):=\inf _{w \in \mathbb{R}^{d}} h^{2}(x, w)+\frac{1}{2 \varepsilon}|w-v|^{2} \quad x, v \in \mathbb{R}^{d}, \varepsilon>0,
$$

which satisfy

$$
0<h_{\varepsilon}^{2}(x, v) \leq \frac{1}{2 \varepsilon}|v|^{2}, \quad \lim _{\varepsilon \downarrow 0} h_{\varepsilon}(x, v)=h(x, v) \quad \text { for every } v \in \mathbb{R}^{d} \backslash\{0\} .
$$

If we define the Finsler distance $\mathrm{d}_{\varepsilon}$ as in (2.33) in terms of $h_{\varepsilon}$ we can easily see that

$$
0<\mathrm{d}_{\varepsilon}^{2}(x, y) \leq \frac{1}{2 \varepsilon}|x-y|^{2} \quad \lim _{\varepsilon \downarrow 0} \mathrm{~d}_{\varepsilon}(x, y)=\mathrm{d}(x, y) \quad \text { for every } x, y \in \mathbb{R}^{d}, x \neq y .
$$

If $\tau$ is the usual Euclidean topology, we obtain that $\left(\mathbb{R}^{d}, \tau, \mathrm{d}\right)$ is an extended metric-topological space. 


\subsection{The Kantorovich-Rubinstein distance}

Let $(X, \tau, \mathrm{d})$ be an extended metric-topological space. We want to lift the same structure to the space of Radon probability measures $\mathcal{P}(X)$. We introduce the main definitions for of couple of measures $\mu_{0}, \mu_{1} \in \mathcal{M}_{+}(X)$ with the same mass $\mu_{0}(X)=\mu_{1}(X)$.

We denote by $\Gamma\left(\mu_{0}, \mu_{1}\right)$ the collection of plans $\boldsymbol{\mu} \in \mathcal{M}_{+}(X \times X)$ whose marginals are $\mu_{0}$ and $\mu_{1}$ respectively:

$$
\Gamma\left(\mu_{0}, \mu_{1}\right):=\left\{\boldsymbol{\mu} \in \mathcal{M}_{+}(X \times X): \pi_{\sharp}^{i} \boldsymbol{\mu}=\mu_{i}\right\}, \quad \pi^{i}\left(x_{0}, x_{1}\right):=x_{i} .
$$

It is not difficult to check that $\Gamma\left(\mu_{0}, \mu_{1}\right)$ is a nonempty (it always contains $\left.\mu_{0}^{-1}(X) \mu_{0} \otimes \mu_{1}\right)$ and compact subset of $\mathcal{M}_{+}(X \times X)$.

Let $\delta: X \times X \rightarrow[0,+\infty]$ be a lower semicontinuous extended semi distance. The Kantorovich formulation of the optimal transport problem with cost $\delta$ induces the celebrated KantorovichRubinstein (extended, semi-)distance $\mathrm{K}_{\delta}$ in $\mathcal{P}(X)$ [64, Chap. 7]

$$
\mathrm{K}_{\delta}\left(\mu_{0}, \mu_{1}\right):=\inf \left\{\int_{X \times X} \delta\left(x_{0}, x_{1}\right) \mathrm{d} \boldsymbol{\mu}\left(x_{0}, x_{1}\right): \boldsymbol{\mu} \in \Gamma\left(\mu_{0}, \mu_{1}\right)\right\} .
$$

Proposition 2.14. Let $\mu_{0}, \mu_{1} \in \mathcal{M}_{+}(X)$ with the same mass.

(a) If $\mathrm{K}_{\delta}\left(\mu_{0}, \mu_{1}\right)$ is finite then the infimum in (2.41) is attained. In particular, this holds if $\delta$ is bounded.

(b) [Kantorovich-Rubinstein duality] If $\delta$ is a bounded continuous (semi)-distance in $(X, \tau)$ then $\mathrm{K}_{\delta}$ is a bounded continuous (semi)-distance in $\mathcal{P}(X)$ and

$$
\begin{aligned}
\mathrm{K}_{\delta}\left(\mu_{0}, \mu_{1}\right)= & \sup \left\{\int \phi_{0} \mathrm{~d} \mu_{0}-\int \phi_{1} \mathrm{~d} \mu_{1}: \phi_{i} \in \mathrm{C}_{b}(X, \tau),\right. \\
& \left.\phi_{0}\left(x_{0}\right)-\phi_{1}\left(x_{1}\right) \leq \delta\left(x_{0}, x_{1}\right) \quad \text { for every } x_{o}, x_{1} \in X\right\} \\
= & \sup \left\{\int \phi \mathrm{d}\left(\mu_{0}-\mu_{1}\right): \phi \in \operatorname{Lip}_{b, 1}(X, \tau, \delta)\right\}
\end{aligned}
$$

(c) If $\left(\mathrm{d}_{i}\right)_{i \in I}$ is a directed collection of bounded continuous semidistances satisfying $\lim _{i \in I} \mathrm{~d}_{i}=\mathrm{d}$ then

$$
\mathrm{K}_{\mathrm{d}}\left(\mu_{0}, \mu_{1}\right)=\lim _{i \in I} \mathrm{~K}_{\mathrm{d}_{i}}\left(\mu_{0}, \mu_{1}\right)
$$

(d) If $(X, \tau, \mathrm{d})$ is an extended metric-topological space

$$
\begin{aligned}
\mathrm{K}_{\mathrm{d}}\left(\mu_{0}, \mu_{1}\right)= & \sup \left\{\int \phi_{0} \mathrm{~d} \mu_{0}-\int \phi_{1} \mathrm{~d} \mu_{1}: \phi_{i} \in \mathrm{C}_{b}(X, \tau),\right. \\
& \left.\phi_{0}\left(x_{0}\right)-\phi_{1}\left(x_{1}\right) \leq \mathrm{d}\left(x_{0}, x_{1}\right) \quad \text { for every } x_{0}, x_{1} \in X\right\} \\
= & \sup \left\{\int \phi \mathrm{d}\left(\mu_{0}-\mu_{1}\right): \phi \in \operatorname{Lip}_{b, 1}(X, \tau, \mathrm{d})\right\}
\end{aligned}
$$

Proof. (a) follows by the lower semicontinuity of $\delta$ and the compactness of $\Gamma$.

(b) we refer to [64, Chap. 7]. 
(c) follows by the property

$$
\liminf _{j \in J} \int \mathrm{d}_{i(j)} \mathrm{d} \boldsymbol{\pi}_{j} \geq \int \mathrm{d} \mathrm{d} \boldsymbol{\pi}
$$

whenever $\left(\boldsymbol{\pi}_{j}\right)_{j \in J}$ is a net in $\Gamma\left(\mu_{0}, \mu_{1}\right)$ converging weakly to $\boldsymbol{\pi}$ and $j \mapsto i(j)$ is a subnet in $I$. See [3, Theorem 5.1]

(d) is an immediate consequence of (2.44) and Claim (b), which yields that $\mathrm{K}_{\mathrm{d}}$ is less or equal than the two expression in the right-hand side of (2.45) and (2.46). The converse inequality is obvious.

Remark 2.15. Thanks to the previous proposition, it would not be difficult to check that $\left(\mathcal{P}(X), \tau_{\mathcal{P}}, \mathrm{K}_{\mathrm{d}}\right)$ is an extended metric-topological space as well.

\subsection{The asymptotic Lipschitz constant}

Whenever $\delta$ is an extended, $\tau$-lower semicontinuous semidistance, and $f: X \rightarrow \mathbb{R}$, we set

$$
\operatorname{lip}_{\delta} f(x):=\lim _{U \in \mathscr{U}_{x}} \operatorname{Lip}(f, U, \delta)=\inf _{U \in \mathscr{U}_{x}} \operatorname{Lip}(f, U, \delta) \quad x \in X
$$

recall that $\mathscr{U}_{x}$ is the directed set of all the $\tau$-neighborhood of $x$. Notice that $\operatorname{Lip}(f,\{x\})=0$ and therefore $\operatorname{lip} f(x)=0$ if $x$ is an isolated point of $X$. We will often omit the index $\delta$ when $\delta=\mathrm{d}$. When $\delta$ is a distance, we can also define $\operatorname{lip}_{\delta}$ as

$$
\operatorname{lip}_{\delta} f(x)=\limsup _{\substack{y, z \rightarrow x \\ y \neq z}} \frac{|f(y)-f(z)|}{\delta(y, z)} ;
$$

in particular,

$$
\operatorname{lip}_{\delta} f(x) \geq\left|\mathrm{D}_{\delta} f\right|(x):=\limsup _{y \rightarrow x} \frac{|f(y)-f(x)|}{\delta(x, y)} .
$$

It is not difficult to check that $x \mapsto \operatorname{lip}_{\delta} f(x)$ is a $\tau$-upper semicontinuous map and $f$ is locally $\delta$ Lipschitz in $X$ iff $\operatorname{lip}_{\delta} f(x)<\infty$ for every $x \in X$. When $(X, \delta)$ is a length space, $\operatorname{lip}_{\delta} f$ coincides with the upper semicontinuous envelope of the local Lipschitz constant (2.50).

We collect in the next useful lemma the basic calculus properties of $\operatorname{lip}_{\delta} f$.

Lemma 2.16. For every $f, g, \chi \in \mathrm{C}_{b}(X)$ with $\chi(X) \subset[0,1]$ we have

$$
\begin{aligned}
\operatorname{lip}_{\delta}(\alpha f+\beta g) & \leq|\alpha| \operatorname{lip}_{\delta} f+|\beta| \operatorname{lip}_{\delta} g \quad \text { for every } \alpha, \beta \in \mathbb{R}, \\
\operatorname{lip}_{\delta}(f g) & \leq|f| \operatorname{lip}_{\delta} g+|g| \operatorname{lip}_{\delta} f, \\
\operatorname{lip}_{\delta}((1-\chi) f+\chi g) & \leq(1-\chi) \operatorname{lip}_{\delta} f+\chi \operatorname{lip}_{\delta} g+\operatorname{lip}_{\delta} \chi|f-g| .
\end{aligned}
$$

Moreover, whenever $\phi \in \mathrm{C}^{1}(\mathbb{R})$

$$
\operatorname{lip}_{\delta}(\phi \circ f)=\left|\phi^{\prime} \circ f\right| \operatorname{lip}_{\delta} f
$$

and for every convex and nondecreasing function $\psi:[0, \infty) \rightarrow \mathbb{R}$ and every map $\zeta \in \mathrm{C}^{1}(\mathbb{R})$ with $0 \leq \zeta^{\prime} \leq 1$, the transformation

$$
\tilde{f}:=f+\zeta(g-f), \quad \tilde{g}:=g+\zeta(f-g)
$$

satisfies

$$
\psi\left(\operatorname{lip}_{\delta} \tilde{f}\right)+\psi\left(\operatorname{lip}_{\delta} \tilde{g}\right) \leq \psi\left(\operatorname{lip}_{\delta} f\right)+\psi\left(\operatorname{lip}_{\delta} g\right) .
$$


Proof. (2.51a) follows by the obvious inequalities

$$
\operatorname{Lip}(\alpha f+\beta g, U, \delta) \leq|\alpha| \operatorname{Lip}(f, U, \delta)+|\beta| \operatorname{Lip}(g, U, \delta)
$$

for every subset $U \subset X$. Similarly, for every $y, z \in U$

$$
\begin{aligned}
|f(y) g(y)-f(z) g(z)| & \leq|(f(y)-f(z)) g(y)|+|(g(y)-g(z)) f(z)| \\
& \leq\left(\operatorname{Lip}(f, U, \delta) \sup _{U}|g|+\operatorname{Lip}(g, U, \delta) \sup _{U}|f|\right) \delta(z, y)
\end{aligned}
$$

and we obtain (2.51b) passing to the limit w.r.t. $U \in \mathscr{U}_{x}$. Setting $\tilde{\chi}:=1-\chi,(2.51 \mathrm{c})$ follows by

$$
\begin{aligned}
\mid \chi(y) f(y) & +\tilde{\chi}(y) g(y)-\chi(z) f(z)+\tilde{\chi}(z) g(z) \mid \\
& \leq|\chi(y)(f(y)-f(z))|+|\tilde{\chi}(y)(g(y)-g(z))|+|(\chi(y)-\chi(z))(f(z)-g(z))| \\
& \leq\left(\sup _{U} \chi \operatorname{Lip}(f, U, \delta)+\sup _{U} \tilde{\chi} \operatorname{Lip}(g, U, \delta)+\sup _{U}|f-g| \operatorname{Lip}(\chi, U, \delta)\right) \delta(y, z)
\end{aligned}
$$

and passing to the limit w.r.t. $U \in \mathscr{U}_{x}$.

Concerning (2.51d), for every $y, z \in U$ we get

$$
|\phi(f(y))-\phi(f(z))| \leq \operatorname{Lip}(\phi, f(U)) \operatorname{Lip}(f, U, \delta) \delta(y, z)
$$

which easily yields $\operatorname{lip}_{\delta} \phi \circ f(x) \leq\left|\phi^{\prime}(f(x))\right| \operatorname{lip}_{\delta} f(x)$. If $\phi^{\prime}(f(x)) \neq 0$, we can find a $\mathrm{C}^{1}$ function $\psi: \mathbb{R} \rightarrow \mathbb{R}$ such that $\psi(\phi(r))=r$ in a neighborhood of $f(x)$, so that the same property yields $\operatorname{lip}_{\delta} f(x) \leq \frac{1}{\left|\phi^{\prime}(f(x))\right|} \operatorname{lip}_{\delta} f \circ \phi(x)$ and the identity in (2.51d).

Let us eventually consider (2.51e). As usual, we consider arbitrary points $y, z \in U, U \in \mathscr{U}_{x}$ obtaining

$$
\begin{aligned}
|\tilde{f}(y)-\tilde{f}(z)| & =|f(y)-f(z)+\zeta(g(y)-f(y))-\zeta(g(z)-f(z))| \\
& =\mid f(y)-f(z)+\alpha((g(y)-g(z)-(f(y)-f(z)) \mid \\
& =|(1-\alpha)(f(y)-f(z))+\alpha(g(y)-g(z))| \\
& \leq((1-\alpha) \operatorname{Lip}(f, U, \delta)+\alpha \operatorname{Lip}(g, U, \delta)) \delta(y, z)
\end{aligned}
$$

for some $\alpha=\alpha_{y, z}=\zeta^{\prime}\left(\theta_{y, z}\right) \in[0,1]$, where $\theta_{y, z}$ is a convex combination of $g(y)-f(y)$ and $g(z)-f(z)$. Passing to the limit w.r.t. $U$ and observing that $\alpha \rightarrow \zeta^{\prime}(g(x)-f(x))$ we get

$$
\operatorname{lip}_{\delta} \tilde{f}(x) \leq\left(1-\zeta^{\prime}(g(x)-f(x))\right) \operatorname{lip}_{\delta} f(x)+\zeta^{\prime}(g(x)-f(x)) \operatorname{lip}_{\delta} g(x) .
$$

A similar argument yields

$$
\operatorname{lip}_{\delta} \tilde{g}(x) \leq\left(1-\zeta^{\prime}(f(x)-g(x))\right) \operatorname{lip}_{\delta} g(x)+\zeta^{\prime}(f(x)-g(x)) \operatorname{lip}_{\delta} f(x) .
$$

Since $\psi$ is convex and nondecreasing, we obtain (2.51e).

\subsection{Compatible algebra of functions}

We have seen in Section 2.2 the important role played by the algebra of function $\operatorname{Lip}_{b}(X, \tau, \mathrm{d})$. In many situations it could be useful to consider smaller subalgebras which are however sufficiently rich to recover the metric properties of an extended metric topological space $(X, \tau, \mathrm{d})$. 
Definition 2.17 (Compatible algebras of Lipschitz functions). Let $\mathscr{A}$ be a unital subalgebra of $\operatorname{Lip}_{b}(X, \tau, \mathrm{d})$ and let us set $\mathscr{A}_{\kappa}:=\mathscr{A} \cap \operatorname{Lip}_{b, \kappa}(X, \tau, \mathrm{d})$.

We say that $\mathscr{A}$ is compatible with the metric-topological structure $(X, \tau, \mathrm{d})$ if

$$
\mathrm{d}(x, y)=\sup _{f \in \mathscr{A}_{1}}|f(x)-f(y)| \quad \text { for every } x, y \in X .
$$

In particular, $\mathscr{A}$ separates the points of $X$.

We say that $\mathscr{A}$ is adapted to $(X, \tau, \mathrm{d})$ if $\mathscr{A}$ is compatible with $(X, \tau, \mathrm{d})$ and it generates the topology $\tau$.

If we do not make a different explicit choice, we will always assume that an e.t.m.m. space $\mathbb{X}$ is endowed with the canonical algebra $\mathscr{A}(\mathbb{X}):=\operatorname{Lip}_{b}(X, \tau, \mathrm{d})$.

Remark 2.18 (Coarser topologies and countably generated algebras). Suppose that $\mathscr{A} \subset \operatorname{Lip}_{b}(X, \tau, \mathrm{d})$ is an algebra compatible with $(X, \tau, \mathrm{d})$ and let $\tau_{\mathscr{A}}$ be the initial topology generated by $\mathscr{A}$ (see A.2 in the Appendix). Then $\left(X, \tau_{\mathscr{A}}, \mathrm{d}\right)$ is an e.m.t. space as well and $\mathscr{A}$ is adapted to $\left(X, \tau_{\mathscr{A}}, \mathrm{d}\right)$; a Radon measure $\mathfrak{m} \in \mathcal{M}_{+}(X, \tau)$ is also Radon in $\left(X, \tau_{\mathscr{A}}\right)$.

This property shows that there is some flexibility in the choice of the topology $\tau$, as long as $\tau$ continuous functions are sufficiently rich to generate the distance $d$. An interesting example occurs when $(X, \tau)$ is a Souslin space. By Remark 2.5 we can always find a countable collection $\left(f_{n}\right)_{n \in \mathbb{N}}$ of $\operatorname{Lip}_{b}(X, \tau, \mathrm{d})$ (or of a compatible algebra $\mathscr{A}$ ) satisfying (2.26). If we denote by $\mathscr{A}^{\prime}$ the algebra generated by the functions $f_{n}, n \in \mathbb{N}$, we obtain a countably generated algebra and an auxiliary topology $\tau^{\prime}=\tau_{\mathscr{A}^{\prime}}$ according to Definition 2.6.

\subsubsection{Examples}

Example 2.19 (Cylindrical functions in Banach spaces and their dual). Let $(X,\|\cdot\|)$ be a Banach space (in particular the space $\mathbb{R}^{d}$ with any norm) endowed with its weak topology (or the dual of a Banach space $B$ with the weak* topology) and let $\mathscr{A}$ be the set of smooth cylindrical functions: a function $f: X \rightarrow \mathbb{R}$ belongs to $\mathscr{A}$ if there exists $\psi \in \mathrm{C}^{\infty}\left(\mathbb{R}^{d}\right)$ with bounded derivatives of every order and $d$ linear functionals $\mathrm{h}_{1}, \cdots, \mathrm{h}_{d} \in X^{\prime}$ (resp. in $B$ if the weak* topology is considered) such that

$$
f(x)=\psi\left(\left\langle\mathrm{h}_{1}, x\right\rangle,\left\langle\mathrm{h}_{2}, x\right\rangle, \cdots,\left\langle\mathrm{h}_{d}, x\right\rangle\right) .
$$

It is not difficult to check check that $\mathscr{A} \subset \operatorname{Lip}_{b}(X, \tau, \mathrm{d})$. In order to approximate the distance $\mathrm{d}(x, y)=\|x-y\|$ between two points in $X$ we can argue as in Example 2.10 by choosing functions of the form $f(x):=\mathrm{t}_{\varepsilon}(\langle\mathrm{h}, x\rangle-\langle\mathrm{h}, y\rangle)$ where $\mathrm{h}$ belongs to the dual (resp. predual) unit ball of $X^{\prime}$ (resp. $B$ ) and $\mathrm{t}_{\varepsilon}(r)$ is a smooth regularization of $\mathrm{t}(r):=0 \vee r \wedge 2\|x-y\|$ coinciding with $r$ in the interval $[\varepsilon,\|x-y\|]$. In the case of Example 2.11 it is sufficient to choose $\mathrm{h}$ in the convex set $L$.

The same approach can be adapted to the "Wiener" construction of Example 2.12: in this case one can use linear functionals in $X^{\prime}$.

In the case $X$ is separable (resp. $X=B^{\prime}$ and $B$ is separable) any Borel (resp. weakly* Borel) measure is Radon.

Example 2.20. A compatible algebra is provided by

$$
\operatorname{Lip}_{b}\left(X, \tau,\left(\mathrm{d}_{i}\right)\right):=\left\{f \in \mathrm{C}_{b}(X, \tau): \exists i \in I: \operatorname{Lip}\left(f, \mathrm{~d}_{i}\right)<\infty\right\}
$$

whenever $\left(\mathrm{d}_{i}\right)_{i \in I}$ is a directed family satisfying $(2.24 \mathrm{a}, \mathrm{b}, \mathrm{c}, \mathrm{d})$. One can also consider the smaller unital algebra of functions generated by the collection of distance functions

$$
\left\{\mathrm{d}_{i}(\cdot, y): y \in X, i \in I\right\} \text {. }
$$


Example 2.21 (Cartesian products). Let us consider two e.t.m. spaces $\left(X^{\prime}, \tau^{\prime}, \mathrm{d}^{\prime}\right)$ and $\left(X^{\prime \prime}, \tau^{\prime \prime}, \mathrm{d}^{\prime \prime}\right)$ with two compatible algebras $\mathscr{A}^{\prime}, \mathscr{A}^{\prime \prime}$. For every $p \in[1,+\infty]$ we can consider the product space $\left(X, \tau, \mathrm{d}_{p}\right)$ where $X=X^{\prime} \times X^{\prime \prime}, \tau$ is the product topology of $\tau^{\prime}$ and $\tau^{\prime \prime}$, and

$$
\begin{aligned}
\mathrm{d}_{p}\left(\left(x^{\prime}, x^{\prime \prime}\right),\left(y^{\prime}, y^{\prime \prime}\right)\right) & :=\left(\mathrm{d}^{\prime}\left(x^{\prime}, y^{\prime}\right)^{p}+\mathrm{d}^{\prime \prime}\left(x^{\prime \prime}, y^{\prime \prime}\right)^{p}\right)^{1 / p} \text { if } p<\infty \\
\mathrm{d}_{\infty}\left(\left(x^{\prime}, x^{\prime \prime}\right),\left(y^{\prime}, y^{\prime \prime}\right)\right) & :=\max \left(\mathrm{d}^{\prime}\left(x^{\prime}, y^{\prime}\right), \mathrm{d}^{\prime \prime}\left(x^{\prime \prime}, y^{\prime \prime}\right)\right)
\end{aligned}
$$

The algebra $\mathscr{A}=\mathscr{A}^{\prime} \otimes \mathscr{A}^{\prime \prime}$ generated by functions $f^{\prime} \in \mathscr{A}^{\prime}$ and $f^{\prime \prime} \in \mathscr{A}^{\prime \prime}$ (an element of $\mathscr{A}$ is a linear combination of functions of the form $\left.f\left(x^{\prime}, x^{\prime \prime}\right):=f^{\prime}\left(x^{\prime}\right) f^{\prime \prime}\left(x^{\prime \prime}\right)\right)$ is compatible with $\left(X, \tau, \mathrm{d}_{p}\right)$. In order to prove that (2.52) holds, let $q$ be the conjugate exponent of $p$ and let us introduce the convex subset of $\mathbb{R}^{2} C_{q}:=\left\{(\alpha, \beta) \in \mathbb{R}^{2}: \alpha^{q}+\beta^{q} \leq 1\right\}$ (with obvious modification when $q=\infty$ ). For every couple of point $\left(x^{\prime}, x^{\prime \prime}\right),\left(y^{\prime}, y^{\prime \prime}\right)$ in $X$ we can find $(\alpha, \beta) \in C_{q}$ such that

$$
\mathrm{d}_{p}\left(\left(x^{\prime}, x^{\prime \prime}\right),\left(y^{\prime}, y^{\prime \prime}\right)\right)=\alpha \mathrm{d}^{\prime}\left(x^{\prime}, y^{\prime}\right)+\beta \mathrm{d}^{\prime \prime}\left(x^{\prime \prime}, y^{\prime \prime}\right) .
$$

It is easy to check that for every $f^{\prime} \in \mathscr{A}_{1}^{\prime}$ and $f^{\prime \prime} \in \mathscr{A}_{1}^{\prime \prime}$ the function $f\left(z^{\prime}, z^{\prime \prime}\right):=\alpha f^{\prime}\left(z^{\prime}\right)+\beta f^{\prime \prime}\left(z^{\prime \prime}\right)$ belongs to $\mathscr{A}_{1}$. Since $\mathscr{A}^{\prime}$ and $\mathscr{A}^{\prime \prime}$ are compatible in the respective spaces, we then get

$$
\begin{aligned}
\mathrm{d}_{p}\left(\left(x^{\prime}, x^{\prime \prime}\right),\left(y^{\prime}, y^{\prime \prime}\right)\right) & =\sup _{f^{\prime} \in \mathscr{A}_{1}^{\prime}, f^{\prime \prime} \in \mathscr{A}_{1}^{\prime \prime}} \alpha\left(f^{\prime}\left(x^{\prime}\right)-f^{\prime}\left(y^{\prime}\right)\right)+\beta\left(f^{\prime \prime}\left(x^{\prime \prime}\right)-f^{\prime \prime}\left(y^{\prime \prime}\right)\right) \\
& =\sup _{f^{\prime} \in \mathscr{A}_{1}^{\prime}, f^{\prime \prime} \in \mathscr{A}_{1}^{\prime \prime}} \alpha f^{\prime}\left(x^{\prime}\right)+\beta f^{\prime \prime}\left(x^{\prime \prime}\right)-\left(\alpha f^{\prime}\left(y^{\prime}\right)+\beta f^{\prime \prime}\left(y^{\prime \prime}\right)\right) .
\end{aligned}
$$

Remark 2.22. The previous example 2.21 shows in particular that the cartesian product of two e.t.m. spaces is also an e.t.m. space, a property that one can also directly check by using the approximating semidistance functions $\left(\mathrm{d}_{i}^{\prime}\right)_{i \in I},\left(\mathrm{~d}_{j}^{\prime \prime}\right)_{j \in J}$.

In order to deal with functions in $\mathscr{A}$ it will be useful to have suitable polynomial approximations of the usual truncation maps.

Lemma 2.23 (Polynomial approximation). Let $c>0, a_{i}, b_{i} \in \mathbb{R}$, and $\phi: \mathbb{R} \rightarrow \mathbb{R}$ be a Lipschitz function satisfying

$$
a_{0} \leq \phi \leq b_{0} \quad \text { in }[-c, c], \quad a_{1} \leq \phi^{\prime} \leq b_{1} \quad \mathscr{L}^{1} \text {-a.e. in }[-c, c] .
$$

There exists a sequence $\left(P_{n}\right)_{n \in \mathbb{N}}$ of polynomials such that

$$
\lim _{n \rightarrow \infty} \sup _{[-c, c]}\left|P_{n}-\phi\right|=0, \quad a_{0} \leq P \leq b_{0}, a_{1} \leq P^{\prime} \leq b_{1} \quad \text { in }[-c, c],
$$

and

$$
\lim _{n \rightarrow \infty}\left|P_{n}^{\prime}(r)-\phi^{\prime}(r)\right|=0 \quad \text { for every } r \in[-c, c] \text { where } \phi \text { is differentiable. }
$$

If moreover $\phi \in \mathrm{C}^{1}([-c, c])$ we also have

$$
\lim _{n \rightarrow \infty} \sup _{r \in[-c, c]}\left|P_{n}^{\prime}(r)-\phi^{\prime}(r)\right|=0 .
$$


Proof. In order to prove the first statement of the lemma, it is sufficient to use the Bernstein polynomials of degree $2 n$ on the interval $[-c, c]$ given by the formula

$$
P_{n}(r):=\frac{1}{(2 c)^{n}} \sum_{k=-n}^{n} \phi(k / n)\left(\begin{array}{c}
2 n \\
k+n
\end{array}\right)(r+c)^{n+k}(c-r)^{n-k}
$$

recalling that $P_{n}$ uniformly converge to $\phi$ in $[-c, c]$ as $n \rightarrow \infty$ and that formula (2.61) preserves the bounds on $\phi$ and $\phi^{\prime}$ [50, Sect. 1.7].

Applying the previous Lemma to the the function $\phi(r):=\alpha \vee r \wedge \beta$ (with $a_{0}=\alpha, b_{0}=\beta$, $a_{1}=0, a_{2}=1$ ), we immediately get the following property.

Corollary 2.24. For every interval $[-c, c], c>0, \alpha, \beta \in \mathbb{R}$ with $\alpha<\beta$, and every $\varepsilon>0$ there exists a polynomial $P_{\varepsilon}=P_{\varepsilon}^{c, \alpha, \beta}$ such that

$$
\begin{gathered}
\left|P_{\varepsilon}(r)-\alpha \vee r \wedge \beta\right| \leq \varepsilon, \quad \alpha \leq P_{\varepsilon}(r) \leq \beta, \quad 0 \leq P_{\varepsilon}^{\prime}(r) \leq 1 \quad \text { for every } r \in[-c, c], \\
\lim _{\varepsilon \downarrow 0} P_{\varepsilon}^{\prime}(r)= \begin{cases}1 & \text { if } \alpha<r<\beta \\
0 & \text { if } r<\alpha \text { or } r>\beta .\end{cases}
\end{gathered}
$$

If $\alpha=-\beta$ we can also find an odd $P_{\varepsilon}$, thus satisfying $P_{\varepsilon}(0)=0$.

A more refined argument yields:

Corollary 2.25. For every interval $[-c, c] \subset \mathbb{R}$ and every $\varepsilon>0$, there exists a polynomial $Q_{\varepsilon}=Q_{\varepsilon}^{c}$ : $\mathbb{R} \times \mathbb{R} \rightarrow \mathbb{R}$ such that

$$
\begin{gathered}
r \wedge s \leq Q_{\varepsilon}(r, s) \leq r \vee s, \quad\left|Q_{\varepsilon}(r, s)-r \vee s\right| \leq \varepsilon \quad \text { for every } r, s \in[-c, c], \\
0 \leq \partial_{r} Q_{\varepsilon} \leq 1, \quad 0 \leq \partial_{s} Q_{\varepsilon} \leq 1 \quad \text { in }[-c, c] \times[-c, c], \\
\left|Q_{\varepsilon}\left(r_{2}, s_{2}\right)-Q_{\varepsilon}\left(r_{1}, s_{1}\right)\right| \leq \max \left(\left|r_{2}-r_{1}\right|,\left|s_{2}-s_{1}\right|\right) \quad \text { for every } r_{i}, s_{i} \in[-c, c] .
\end{gathered}
$$

Proof. We apply Lemma 2.23 to the function $\phi(r):=r_{+}$in the interval $[-4 c, 4 c]$ (with $a_{0}=a_{1}=0$, $b_{0}=4 c$ and $\left.b_{1}=1\right)$ obtaining a polynomial $P_{\varepsilon}$ such that

$$
\left|P_{\varepsilon}(r)-r_{+}\right| \leq \varepsilon, \quad 0 \leq P_{\varepsilon}(r) \leq 4 c, \quad 0 \leq P_{\varepsilon}^{\prime}(r) \leq 1 \quad \text { for every } r \in[-4 c, 4 c] .
$$

We set $Q_{\varepsilon}(r, s):=r+P_{\varepsilon}(s-r)-P_{\varepsilon}(0)$. Notice that $Q_{\varepsilon}$ is increasing w.r.t. $r, s$ in $[-2 c, 2 c] \times[-2 c, 2 c]$ since

$$
\partial_{r} Q_{\varepsilon}(r, s)=1-P_{\varepsilon}^{\prime}(s-r) \geq 0, \quad \partial_{s} Q_{\varepsilon}(r, s)=P_{\varepsilon}^{\prime}(s-r) \geq 0
$$

in particular

$$
Q_{\varepsilon}(r, s) \geq Q_{\varepsilon}(r \wedge s, r \wedge s)=r \wedge s, \quad Q_{\varepsilon}(r, s) \leq Q_{\varepsilon}(r \vee s, r \vee s)=r \vee s .
$$

By construction, if $r, s \in[-c, c]$ then

$$
\left|Q_{\varepsilon}(r, s)-r \vee s\right|=\left|r+P_{\varepsilon}(s-r)-\left(r+(s-r)_{+}\right)\right|=\left|P_{\varepsilon}(s-r)-(s-r)_{+}\right| \leq \varepsilon .
$$

Concerning the Lipschitz estimate, let us consider points $\left(r_{1}, s_{1}\right),\left(r_{2}, s_{2}\right) \in[-c, c]$. Up to inverting the order of the couples, it is not restrictive to assume that $Q_{\varepsilon}\left(r_{1}, s_{1}\right) \geq Q_{\varepsilon}\left(r_{2}, s_{2}\right)$. Setting $r_{-}:=$ 
$r_{1} \wedge r_{2}, r_{+}:=r_{1} \vee r_{2}, s_{-}:=s_{1} \wedge s_{2}, s_{+}:=s_{1} \vee s_{2}, \bar{r}:=\left(r_{+}-r_{-}\right) \leq 2 c, \bar{s}=s_{+}-s_{-} \leq 2 c$, $\bar{z}:=\bar{r} \vee \bar{s}=\max \left(\left|r_{2}-r_{1}\right|,\left|s_{2}-s_{1}\right|\right) \leq 2 c$, the partial monotonicity of $Q_{\varepsilon}$ yields

$$
\begin{aligned}
\left|Q_{\varepsilon}\left(r_{1}, s_{1}\right)-Q_{\varepsilon}\left(r_{2}, s_{2}\right)\right| & \leq Q_{\varepsilon}\left(r_{+}, s_{+}\right)-Q_{\varepsilon}\left(r_{-}, s_{-}\right) \leq Q_{\varepsilon}\left(r_{-}+\bar{z}, s_{-}+\bar{z}\right)-Q_{\varepsilon}\left(r_{-}, s_{-}\right) \\
& =\int_{0}^{\bar{z}}\left(\partial_{r} Q_{\varepsilon}\left(r_{-}+z, s_{-}+z\right)+\partial_{s} Q_{\varepsilon}\left(r_{-}+z, s_{-}+z\right)\right) \mathrm{d} z \\
& =\int_{0}^{\bar{z}}\left(1-P_{\varepsilon}^{\prime}\left(s_{-}-r_{-}\right)+P_{\varepsilon}^{\prime}\left(s_{-}-r_{-}\right)\right) \mathrm{d} z=\bar{z} .
\end{aligned}
$$

The next result shows how to obtain good approximations of the maximum of a finite number of functions in $\mathscr{A}$.

Lemma 2.26. Let $f^{1}, f^{2}, \cdots, f^{M} \in \mathscr{A}$ and let $f:=\max \left(f^{1}, f^{2}, \cdots, f^{M}\right)$. Then for every $\varepsilon>0$ there exists a sequence $f_{\varepsilon} \in \mathscr{A}$ such that

$$
\min _{m} f^{m}(x) \leq f_{\varepsilon}(x) \leq \max _{m} f^{m}(x) \quad \text { for every } x \in X, \quad \sup _{X}\left|f_{\varepsilon}-f\right| \leq \varepsilon .
$$

If moreover $\operatorname{Lip}\left(f^{m}, A, \delta\right) \leq L$ for $1 \leq m \leq M$ where $A \subset X$ and $\delta$ is an extended semidistance on $X$, then $\operatorname{Lip}\left(f_{\varepsilon}, A, \delta\right) \leq L$ for every $n \in \mathbb{N}$.

Proof. We split the proof in two steps.

1. The thesis of the Lemma holds for $M=2$. We set $c>0$ so that $f^{m}(X) \subset[-c, c]$ and then we define $f_{\varepsilon}:=Q_{\varepsilon}\left(f^{1}, f^{2}\right)$, where $Q_{\varepsilon}$ has been provided by Corollary 2.25. (2.67) follows immediately by (2.63). (2.65) yields for every $x, y \in X$

$\left|f_{\varepsilon}(x)-f_{\varepsilon}(y)\right|=\left|Q_{\varepsilon}\left(f^{1}(x), f^{2}(x)\right)-Q_{\varepsilon}\left(f^{1}(y), f^{2}(y)\right)\right| \leq \max \left(\left|f^{1}(x)-f^{1}(y)\right|,\left|f^{2}(x)-f^{2}(y)\right|\right)$

so that the composition with $Q_{\varepsilon}$ preserve the Lipschitz constant w.r.t. arbitrary sets and semidistances.

2. The thesis of the Lemma holds for arbitrary $M \in \mathbb{N}$. We argue by induction, assuming that the result is true for $M-1$. We fix a constant $c$ so that $f^{m}(X) \subset[-c, c]$ for $1 \leq m \leq M$. We thus find $h_{\varepsilon / 2} \subset \mathscr{A}$ satisfying

$$
\min _{1 \leq m \leq M-1} f^{m}(x) \leq h_{\varepsilon / 2}(x) \leq \max _{1 \leq m \leq M-1} f^{m}(x) \quad \text { for every } x \in X, \quad \sup _{X}\left|h_{\varepsilon / 2}-\tilde{f}\right| \leq \varepsilon / 2,
$$

where $\tilde{f}:=f^{1} \vee \cdots \vee f^{M-1}$; in particular $h_{\varepsilon / 2}(X) \subset[-c, c]$. We then set $f_{\varepsilon}:=Q_{\varepsilon / 2}\left(h_{\varepsilon / 2}, f^{M}\right)$; clearly for every $x \in X$

$$
\min _{1 \leq m \leq M} f^{m}(x) \leq h_{\varepsilon / 2}(x) \wedge f^{M}(x) \leq f_{\varepsilon}(x) \leq h_{\varepsilon / 2}(x) \vee f^{M}(x) \leq \max _{1 \leq m \leq M} f^{m}(x) ;
$$

moreover

$$
\begin{aligned}
\left|f_{\varepsilon}-f\right| & \leq\left|Q_{\varepsilon / 2}\left(h_{\varepsilon / 2}, f^{M}\right)-f\right| \\
& \leq\left|Q_{\varepsilon / 2}\left(h_{\varepsilon / 2}, f^{M}\right)-Q_{\varepsilon / 2}\left(\tilde{f}, f^{M}\right)\right|+\left|Q_{\varepsilon / 2}\left(\tilde{f}, f^{M}\right)-\tilde{f} \vee f^{M}\right| \\
& \leq\left|h_{\varepsilon / 2}-\tilde{f}\right|+\varepsilon / 2 \leq \varepsilon / 2+\varepsilon / 2 \leq \varepsilon .
\end{aligned}
$$

We conclude this section by a simple density results that will be useful in the following. 
Lemma 2.27 (Density of $\mathscr{A}$ in $L^{p}(X, \mathfrak{m})$ ). Let $p \in[1,+\infty)$ and let I a closed (possibly unbounded) interval of $\mathbb{R}$. If $\mathscr{A}$ is a compatible sub-algebra of $\operatorname{Lip}_{b}(X, \tau, \mathrm{d})$, then for every $f \in \mathcal{L}^{p}(X, \mathfrak{m})$ with values in I there exists a sequence $f_{n} \in \mathscr{A}$ with values in I such that $\int_{X}\left|f-f_{n}\right|^{p} \mathrm{dm} \rightarrow 0$.

Proof. By standard approximation, it is not restrictive to assume that $I=[\alpha, \beta]$ for some $\alpha, \beta \in \mathbb{R}$; we set $\gamma:=|\alpha| \vee|\beta|$. Since $\mathfrak{m}$ is Radon, every $\mathfrak{m}$-measurable function $f$ is Lusin $\mathfrak{m}$-measurable: thus for every $\varepsilon>0$ there exists a compact $K \subset X$ such that $f_{\left.\right|_{K}}$ is continuous and $\mathfrak{m}(X \backslash K) \leq \varepsilon$.

Since $\mathscr{A}$ contains the constants and separates the points of $K$, the restriction of $\mathscr{A}$ to $K$ is uniformly dense in $\mathrm{C}_{b}(K, \tau)$ by Stone-Weierstrass Theorem: we thus find $\tilde{f}_{\varepsilon} \in \mathscr{A}$ such that $\sup _{x \in K} \mid f(x)-$ $\tilde{f}_{\varepsilon}(x) \mid \leq \varepsilon$. If $c:=\sup _{X}\left|\tilde{f}_{\varepsilon}\right| \vee \gamma$, applying Corollary 2.24 we can find a polynomial $P_{\varepsilon}$ satisfying (2.62), so that $f_{\varepsilon}:=P_{\varepsilon} \circ \tilde{f}_{\varepsilon}$ belongs to $\mathscr{A}$, takes values in $[\alpha, \beta]$, and satisfies

$$
\left|f_{\varepsilon}-f\right| \leq\left|P_{\varepsilon}\left(\tilde{f}_{\varepsilon}\right)-P_{\varepsilon}(f)\right|+\left|P_{\varepsilon}(f)-f\right| \leq 2 \varepsilon \quad \text { in } K,
$$

so that

$$
\int_{X}\left|f_{\varepsilon}-f\right|^{p} \mathrm{~d} \mathfrak{m} \leq(2 \varepsilon)^{p} \mathfrak{m}(X)+(\beta-\alpha)^{p} \varepsilon
$$

Choosing a sequence $f_{n}:=f_{\varepsilon_{n}}$ corresponding to a vanishing sequence $\varepsilon_{n} \downarrow 0$ we conclude.

\subsection{Embeddings and compactification of extended metric-measure spaces}

Let $\mathbb{X}=(X, \tau, \mathrm{d}, \mathfrak{m})$ and $\mathbb{X}^{\prime}=\left(X^{\prime}, \tau^{\prime}, \mathrm{d}^{\prime}, \mathfrak{m}^{\prime}\right)$ be two extended metric measure spaces, endowed with compatible algebras $\mathscr{A}, \mathscr{A}^{\prime}$ according to definition 2.17.

Definition 2.28 (Embedding, compactification, and isomorphism). We say that a map $\iota: X \rightarrow X^{\prime}$ is a measure-preserving embedding of $(\mathbb{X}, \mathscr{A})$ into $\left(\mathbb{X}^{\prime}, \mathscr{A}^{\prime}\right)$ if

(E1) $\iota$ is a continuous and injective map of $(X, \tau)$ into $\left(X^{\prime}, \tau^{\prime}\right)$;

(E2) $\iota$ is an isometry, in the sense that

$$
\mathrm{d}^{\prime}(\iota(x), \iota(y))=\mathrm{d}(x, y) \quad \text { for every } x, y \in X .
$$

(E3) $\iota_{\sharp} \mathfrak{m}=\mathfrak{m}^{\prime}$.

(E4) For every $f^{\prime} \in \mathscr{A}^{\prime}$ the function $\iota^{*} f^{\prime}:=f^{\prime} \circ \iota$ belongs to $\mathscr{A}$.

We say that $\left(\mathbb{X}^{\prime}, \mathscr{A}^{\prime}\right)$ is a compactification of $(\mathbb{X}, \mathscr{A})$ if $\left(X^{\prime}, \tau^{\prime}\right)$ is compact and there exists a measurepreserving embedding of $(\mathbb{X}, \mathscr{A})$ into $\left(\mathbb{X}^{\prime}, \mathscr{A}^{\prime}\right)$.

We say that a measure-preserving embedding $\iota$ is an isomorphism of $(\mathbb{X}, \mathscr{A})$ onto $(\hat{\mathbb{X}}, \hat{\mathscr{A}})$ if $\iota$ is an homeomorphism of $(X, \tau)$ onto $(\hat{X}, \hat{\tau})$ and $\iota^{*}\left(\mathscr{A}^{\prime}\right)=\mathscr{A}$.

Remark 2.29 (Canonical Lipschitz algebra). When $\mathscr{A}=\operatorname{Lip}_{b}(X, \tau, \mathrm{d})$ and $\mathscr{A}^{\prime}=\operatorname{Lip}_{b}\left(X^{\prime}, \tau^{\prime}, \mathrm{d}^{\prime}\right)$ we simply say that $\iota$ is a measure preserving embedding of $\mathbb{X}$ into $\mathbb{X}^{\prime}$. In this case it is sufficient to check conditions (E1-2-3), since condition (E4) is a consequence of (E1-2). In this case $\iota$ is an isomorphism of $(\mathbb{X}, \mathscr{A})$ onto $\left(\iota(X), \tau^{\prime}, \mathrm{d}^{\prime}, \mathfrak{m}^{\prime}\right)$.

Example 2.30. Let us show three simple examples of embeddings involving an e.m.t.m. space $\mathbb{X}=$ $(X, \tau, \mathrm{d}, \mathfrak{m})$.

(a) Let $\tau^{\prime}$ be a weaker topology than $\tau$, such that $\left(X, \tau^{\prime}, \mathrm{d}\right)$ is an e.t.m. space. The identity map provides a measure-preserving embedding of $\mathbb{X}$ into $\left(X, \tau^{\prime}, \mathrm{d}, \mathfrak{m}\right)$. 
(b) Let $\mathscr{A}^{\prime} \subset \mathscr{A}$ be two compatible sub-algebras of $\mathbb{X}$. The identity map provides a measurepreserving embedding of $(\mathbb{X}, \mathscr{A})$ into $\left(\mathbb{X}, \mathscr{A}^{\prime}\right)$ (in particular when $\mathscr{A}=\operatorname{Lip}_{b}(X, \tau, \mathrm{d})$ ).

(c) By Corollary 2.7, if $(X, \tau)$ is a Souslin space, one can always find a measure preserving embedding in the space $\left(X, \tau^{\prime}, \mathscr{A}^{\prime}, \mathrm{d}\right)$ where $\mathscr{A}^{\prime} \subset \mathscr{A}$ is countably generated and $\tau^{\prime}$ is an auxiliary topology (thus metrizable and separable, coarser than $\tau$ ).

(d) Let $Y$ be any $\mathfrak{m}$-measurable subset of $X$ such that $\mathfrak{m}(X \backslash Y)=0$; we denote by $\tau_{Y}$ the relative topology of $Y, \mathrm{~d}_{Y}$ the restriction of $\mathrm{d}$ to $Y \times Y$ and $\mathfrak{m}_{Y}:=\left.\mathfrak{m}\right|_{Y}$. If $\mathscr{A}$ is a compatible algebra for $\mathbb{X}$ we define $\mathscr{A}_{Y}:=\left\{f_{\left.\right|_{Y}}: f \in \mathscr{A}\right\}$ as the algebra obtained by the restriction to $Y$ of the elements of $\mathscr{A}$. It is easy to check that $\mathbb{Y}=\left(Y, \tau_{Y}, \mathrm{~d},\left.\mathfrak{m}\right|_{Y}\right)$ is an e.m.t.m. space with a compatible algebra $\mathscr{A}_{Y}$ and the inclusion map $\iota: Y \rightarrow X$ is a measure-preserving embedding of $\left(\mathbb{Y}, \mathscr{A}_{Y}\right)$ into $(\mathbb{X}, \mathscr{A})$.

Let us collect a few simple results concerning the corresponding between measurable functions induced by a measure-preserving embedding. Whenever $f^{\prime}: X^{\prime} \rightarrow \mathbb{R}$ we write

$$
f=\iota^{*} f^{\prime}:=f^{\prime} \circ \iota \text {. }
$$

Note that if $\iota(X)$ is $\tau^{\prime}$-dense in $X^{\prime}$ the pull back map $\iota^{*}$ is injective. Independently from this property, we will show that $\iota^{*}$ induces an isomorphism between classes of measurable functions identified by $\mathfrak{m}^{\prime}$ and $\mathfrak{m}$-a.e. equivalence respectively. We write $f_{1} \sim_{\mathfrak{m}} f_{2}$ if $\mathfrak{m}\left(\left\{f_{1} \neq f_{2}\right\}\right)=0$ and we will denote by $[f]_{\mathfrak{m}}$ the equivalence class of a $\mathfrak{m}$-measurable map $f$.

Lemma 2.31. Let $\iota: X \rightarrow X^{\prime}$ be a measure-preserving embedding of $(\mathbb{X}, \mathscr{A})$ into $\left(\mathbb{X}^{\prime}, \mathscr{A}^{\prime}\right)$ according to the previous definition.

(a) For every $\mathfrak{m}^{\prime}$-measurable function $f^{\prime}: X^{\prime} \rightarrow \mathbb{R}$ the function $\iota^{*} f^{\prime}$ is $\mathfrak{m}$-measurable and we have

$$
f_{1}^{\prime} \sim_{\mathfrak{m}^{\prime}} f_{2}^{\prime} \quad \Leftrightarrow \quad \iota^{*} f_{1}^{\prime} \sim_{\mathfrak{m}} \iota^{*} f_{2}^{\prime}
$$

(b) The algebra $\mathscr{A}^{*}:=\iota^{*}\left(\mathscr{A}^{\prime}\right)$ is a sub-algebra of $\mathscr{A}$ which is compatible with the extended metric-measure space $\mathbb{X}$.

(c) For every $p \in[1,+\infty] \iota^{*}$ induces a linear isomorphism between $L^{p}\left(X^{\prime}, \mathfrak{m}^{\prime}\right)$ and $L^{p}(X, \mathfrak{m})$, whose inverse is denoted by $\iota_{*}$. For every $f \in \mathscr{A}^{*} / \sim_{\mathfrak{m}}$ the class $\iota_{*} f$ contains all the elements $f^{\prime} \in \mathscr{A}^{\prime}$ satisfying $\iota^{*} f^{\prime}=f$.

Proof. The proof of (a) is immediate: (2.72) is a consequence of the fact that the set $N^{\prime}:=\left\{f_{1}^{\prime} \neq f_{2}^{\prime}\right\}$ satisfies

$$
\iota^{-1} N^{\prime}=\left\{\iota^{*} f_{1} \neq \iota^{*} f_{2}\right\}, \quad \mathfrak{m}\left(\iota^{-1} N^{\prime}\right)=\mathfrak{m}^{\prime}(N) .
$$

(b) It is immediate to check that $\mathscr{A}^{*}$ is a unital algebra included in $\mathscr{A}$. It is not difficult to check that $\mathscr{A}^{*}$ satisfies (2.52): by (2.70) if $f^{\prime} \in \mathscr{A}_{1}^{\prime}$ then $\iota^{*} f^{\prime} \in \mathscr{A}_{1}^{*}$ and since $\mathscr{A}^{\prime}$ is compatible with $\mathbb{X}^{\prime}$, for every $x, y \in X$

$$
\begin{aligned}
\mathrm{d}(x, y) \stackrel{(2.70)}{=} & \mathrm{d}^{\prime}(\iota(x), \iota(y))=\sup _{f^{\prime} \in \mathscr{A}_{1}^{\prime}}\left|f^{\prime}(\iota(x))-f^{\prime}(\iota(y))\right|=\sup _{f^{\prime} \in \mathscr{A}_{1}^{\prime}}\left|\iota^{*} f^{\prime}(x)-\iota^{*} f^{\prime}(y)\right| \\
& =\sup _{f \in \mathscr{A}_{1}^{*}}|f(x)-f(y)| .
\end{aligned}
$$


(c) Thanks to property (E3) of Definition 2.28 we have

$$
\int_{X^{\prime}} \Phi\left(f^{\prime}\right) \mathrm{d} \mathfrak{m}^{\prime}=\int_{X} \Phi\left(f^{\prime} \circ \iota\right) \mathrm{d} \mathfrak{m}=\int_{X} \Phi\left(\iota^{*} f^{\prime}\right) \mathrm{d} \mathfrak{m}
$$

for every nonnegative continuous function $\Phi: \mathbb{R} \rightarrow[0,+\infty)$, so that $\iota^{*}$ induces a linear isometry from each $L^{p}\left(X^{\prime}, \mathfrak{m}^{\prime}\right)$ into $L^{p}(X, \mathfrak{m})$. It is therefore sufficient to prove that $\iota^{*}$ is surjective; since $\iota^{*}$ is an isometry with respect to the $L^{1}$-norm, this is equivalent to the density of the image of $\iota^{*}$ in $L^{1}(X, \mathfrak{m})$. Since the image contains (the equivalence classes of elements in) $\mathscr{A}^{*}$, the density follows by (b) and Lemma 2.27. The last statement is a consequence of (2.72).

On of the most useful application of the concept of measure-preserving embeddings is the possibility to construct a compactification $\mathbb{X}^{\prime}$ of $\mathbb{X}$ starting from a compatible algebra $\mathscr{A}$. As a byproduct, we will obtain a compatible algebra $\mathscr{A}^{\prime}$ in $\mathbb{X}^{\prime}$ such that

$$
f^{\prime} \in \mathscr{A}^{\prime} \Leftrightarrow f=f^{\prime} \circ \iota \in \mathscr{A} .
$$

As a general fact, every completely regular space $(X, \tau)$ can be homeomorphically imbedded as a dense subset of a compact Hausdorff space $\beta X$ (called the Stone-Cech compactification, [54, $\S 38]$ ), where every function $f \in \mathrm{C}_{b}(X)$ admits a unique continuous extension. The Gelfand theory of Banach algebras applied to $\mathrm{C}_{b}(X)$ provides one of the most effective construction of such a compactification and has the advantage to be well adapted to the setting of extended metric-topological spaces and compatible sub-algebras.

Let us briefly recall the construction. We consider $\mathscr{A}$ as a vector subspace of $\mathrm{C}_{b}(X, \tau)$ endowed with the sup norm $\|\cdot\|_{\infty}$ and we call $\overline{\mathscr{A}}$ the (strong) closure of $\mathscr{A}$ in $\mathrm{C}_{b}(X, \tau)$. Since $\left(\mathscr{A},\|\cdot\|_{\infty}\right)$ is a normed space we can consider the dual Banach space $\left(\mathscr{A}^{*},\|\cdot\|_{\mathscr{A}^{*}}\right)$ endowed with the weak ${ }^{*}$ topology $\hat{\tau}$ and the distinguished subset of characters.

Definition 2.32 (Characters). A character of $\mathscr{A}$ is an element $\varphi$ of $\mathscr{A}^{*} \backslash\{0\}$ satisfying

$$
\varphi(f g)=\varphi(f) \varphi(g) \text { for every } f, g \in \mathscr{A} .
$$

We will denote by $\hat{X}$ the subset of the characters of $\mathscr{A}$.

Let us first recall a preliminary list of useful properties of $\hat{X}$.

Proposition 2.33. Let us consider the set

$$
\Sigma:=\left\{\psi \in \mathscr{A}^{*}:\|\psi\|_{\mathscr{A}^{*}} \leq 1, \psi(f) \geq 0 \text { for every } f \geq 0, \psi(\mathbb{1})=1\right\} .
$$

(a) $\Sigma$ is a weakly* compact convex subset of $\mathscr{A}^{*}$ contained in $\left\{\psi \in \mathscr{A}^{*}:\|\psi\|_{\mathscr{A}^{*}}=1\right\}$.

(b) $\hat{X}$ is a (weakly*) compact subset of $\Sigma$.

(c) Every point of $\hat{X}$ is an extremal point of $\Sigma$.

Proof. (a) is an immediate consequence of Banach-Alaouglu-Bourbaki theorem.

(b) It is not difficult to check that every element $f \in \mathscr{A}$ with $0<m \leq f \leq M$ admits a nonnegative square root $g \in \overline{\mathscr{A}}$ such that $g^{2}=f:$ it is sufficient to define $h:=1-f / M \in \mathscr{A}$ taking values in $[0,1-m / M]$ and use the power series expansion of the square root function in $] 0,2[$ :

$$
g=\sqrt{M} \sum_{n=0}^{\infty}\left(\begin{array}{c}
1 / 2 \\
n
\end{array}\right)(-1)^{n} h^{n}=\sum_{n=0}^{\infty}\left(\begin{array}{c}
1 / 2 \\
n
\end{array}\right) M^{1 / 2-n}(f-M)^{n} .
$$


The relation $\varphi(f)=(\varphi(g))^{2}$ shows that $\varphi f \geq 0$ and since every nonnegative $f \in \mathscr{A}$ can be strongly approximated by uniformly positive elements we obtain that every $\varphi \in \hat{X}$ satisfies $\varphi(f) \geq 0$ for every nonnegative $f \in \mathscr{A}$. Moreover, since $\varphi \neq 0$, there exists an element $f \in \mathscr{A}$ such that $\varphi(f) \neq 0$; from $0 \neq \varphi(f)=\varphi(f \mathbb{1})=\varphi(f) \varphi(\mathbb{1})$ we deduce that $\varphi(\mathbb{1})=1$. By comparison we obtain

$$
\varphi(\gamma \mathbb{1})=\gamma \quad \text { for every } \gamma \in \mathbb{R} ; \quad-\alpha \leq f \leq \beta \quad \Rightarrow \quad-\alpha \leq \varphi(f) \leq \beta,
$$

and in particular

$$
-\|f\|_{\infty} \leq \varphi(f) \leq\|f\|_{\infty},
$$

so that every element of $\hat{X}$ is included in the weakly* compact set $\Sigma$. Since condition (2.75) characterize a weakly* closed set, we conclude that $\hat{X}$ is a compact Hausdorff space endowed with the weak* topology of $\mathscr{A}^{*}$.

(c) Whenever $\psi \in \Sigma$ and $f \in \mathscr{A}$, the inequality

$$
0 \leq \psi\left((f+\kappa \mathbb{1})^{2}\right)=\psi\left(f^{2}\right)+2 \kappa \psi(f)+\kappa^{2} \psi(\mathbb{1})=\psi\left(f^{2}\right)+2 \kappa \psi(f)+\kappa^{2}
$$

for every $\kappa \in \mathbb{R}$ shows that

$$
(\psi(f))^{2} \leq \psi\left(f^{2}\right) \quad \text { for every } \psi \in \Sigma
$$

If $\hat{X} \ni \varphi=\frac{1}{2} \varphi_{1}+\frac{1}{2} \varphi_{2}$ with $\varphi_{i} \in \Sigma$, we obtain

$$
\begin{aligned}
\frac{1}{2} \varphi_{1}\left(f^{2}\right)+\frac{1}{2} \varphi_{2}\left(f^{2}\right) & =\varphi\left(f^{2}\right)=(\varphi(f))^{2}=\frac{1}{4}\left(\varphi_{1}(f)\right)^{2}+\frac{1}{4}\left(\varphi_{2}(f)\right)^{2}+\frac{1}{2} \varphi_{1}(f) \varphi_{2}(f) \\
& \leq \frac{1}{4} \varphi_{1}\left(f^{2}\right)+\frac{1}{4} \varphi_{2}\left(f^{2}\right)+\frac{1}{2} \varphi_{1}(f) \varphi_{2}(f)
\end{aligned}
$$

thus showing that

$$
\left(\frac{1}{2} \varphi_{1}(f)-\frac{1}{2} \varphi_{2}(f)\right)^{2}=\frac{1}{4} \varphi_{1}\left(f^{2}\right)+\frac{1}{4} \varphi_{2}\left(f^{2}\right)-\frac{1}{2} \varphi_{1}(f) \varphi_{2}(f) \leq 0
$$

for every $f \in \mathscr{A}$ and therefore $\varphi_{1}=\varphi_{2}$.

We can now define a canonical embedding $\iota: X \rightarrow \hat{X}$ by

$$
\iota(x)=\hat{x}, \quad \hat{x}(f):=f(x) \quad \text { for every } x \in X, f \in \mathscr{A},
$$

and we call $\Gamma: \overline{\mathscr{A}} \rightarrow \mathrm{C}_{b}(\hat{X}, \hat{\tau})$ the Gelfand transform

$$
\hat{f}=\Gamma f, \quad \hat{f}(\varphi):=\varphi(f) \quad \text { for every } \varphi \in \hat{X} .
$$

We will set $\hat{\mathscr{A}}:=\Gamma(\mathscr{A})$.

Theorem 2.34 (Gelfand compactification of extended metric topological measure spaces).

(a) $\iota$ is a continuous and injective, $\iota(X)$ is a dense subset of the compact Hausdorff space $\hat{X}$ endowed with the weak ${ }^{*}$ topology $\hat{\tau}$. If $\mathscr{A}$ is separable w.r.t. the uniform norm then $\hat{X}$ is metrizable.

(b) $\iota$ is a homeomorphism between $X$ and $\iota(X)$ if and only if $\mathscr{A}$ is adapted, i.e. it generates the topology $\tau$. 
(c) Every function $f \in \overline{\mathscr{A}}$ admits a unique extension $\hat{f}=\Gamma f$ to $\hat{X}$ and the unital algebra $\hat{\mathscr{A}}:=$ $\Gamma(\mathscr{A})$ is uniformly dense in $\mathrm{C}_{b}(\hat{X}, \hat{\tau})$. The pull back algebra $\mathscr{A}^{*}$ coincides with $\mathscr{A}$.

(d) The measure $\hat{\mathfrak{m}}:=\iota_{\sharp} \mathfrak{m}$ is a Radon measure on $\hat{X}$ concentrated on the $\hat{\mathfrak{m}}$-measurable subset $\iota(X)$.

(e) If I is the directed set of all the finite collections of functions in $\mathscr{A}_{1}$, setting

$$
\begin{aligned}
\hat{\mathrm{d}}_{i}\left(\varphi_{1}, \varphi_{2}\right) & :=\sup _{f \in i}\left|\varphi_{1}(f)-\varphi_{2}(f)\right|=\sup _{f \in i}\left|\hat{f}_{i}\left(\varphi_{1}\right)-\hat{f}_{i}\left(\varphi_{2}\right)\right|, \\
\hat{\mathrm{d}}\left(\varphi_{1}, \varphi_{2}\right) & :=\sup _{f \in \mathscr{A}_{1}}\left|\varphi_{1}(f)-\varphi_{2}(f)\right|=\sup _{i \in I} \hat{\mathrm{d}}_{i}\left(\varphi_{1}, \varphi_{2}\right),
\end{aligned}
$$

$\hat{\mathrm{d}}_{i}$ are continuous and bounded semidistances on $\hat{X}$ and $\mathrm{d}$ is an extended distance on $\hat{X}$ satisfying

$$
\hat{\mathrm{d}}_{i}(\hat{x}, \hat{y})=\mathrm{d}_{i}(x, y), \quad \hat{\mathrm{d}}(\hat{x}, \hat{y})=\mathrm{d}(x, y) \quad \text { for every } x, y \in X .
$$

(f) $\iota$ is a measure preserving embedding of $(\mathbb{X}, \mathscr{A})$ into the compact extended metric measure space $\mathbb{X}^{\prime}:=(\hat{X}, \hat{\tau}, \hat{\mathrm{d}}, \hat{\mathfrak{m}})$ endowed with the compatible algebra $\hat{\mathscr{A}}=\Gamma(\mathscr{A})$.

(g) The map $\iota_{*}: L^{p}(X, \mathfrak{m}) \rightarrow L^{p}(\hat{X}, \hat{\mathfrak{m}})$ is the unique linear isometric extension of $\Gamma: \mathscr{A} \rightarrow \hat{\mathscr{A}}$ to $L^{p}(X, \mathfrak{m})$ for every $p \in[1, \infty[$.

Proof. (a) Since the weak* topology is the coarsest topology that makes all the maps $\varphi \mapsto \varphi(f)$ continuous for every $f \in \mathscr{A}$, the continuity of $\iota$ is equivalent to check the continuity of $x \mapsto f(x)$ from $(X, \tau)$ to $\mathbb{R}$, which is guaranteed by the $\tau$-continuity of $f . \iota$ is also injective, since $\mathscr{A}$ separates the points of $X$.

Let us now prove that $\iota(X)$ is dense in $\hat{X}$. Let us denote by $Y$ the weak ${ }^{*}$ closure of $\iota(X)$ and let us first consider the closed convex hull $K:=\overline{\operatorname{co}(Y)}$ of $Y$ in the weak ${ }^{*}$ topology. Since $K$ is bounded and weakly* closed, $K$ is compact. If a point $\psi \in \hat{X}$ does not belong to $K$ we can apply the second geometric form of Hahn-Banach Theorem [56, Thm. 3.21] to find $f \in \overline{\mathscr{A}}$ separating $\psi$ from $K$ : there exists $\alpha \in \mathbb{R}$ such that $\zeta(f) \geq \alpha>\psi(f)$ for every $\zeta \in K$. Choosing $\zeta=\hat{z}=\iota(z)$ for $z \in X$ we deduce that $f(z) \geq \alpha$ for every $z \in X$ and therefore $\psi(f) \geq \alpha$ since $\psi$ is a nonnegative functional.

Thus $\psi \in \overline{\operatorname{co}(Y)}$; since $Y \subset \Sigma$, also $\overline{\operatorname{co}(Y)} \subset \Sigma$; since $\psi$ is an extreme point of $\Sigma$, we deduce that $\psi$ is an extreme point of $\overline{\operatorname{co}(Y)}$; applying Milman's theorem [56, Thm. 3.25] we conclude that $\psi \in Y$.

Finally, if $\mathscr{A}$ is separable then the unit ball of $\mathscr{A}^{*}$ endowed with the weak* topology is metrizable so that $\hat{X}$ is metrizable as well.

(b) It is easy to check that $\hat{f} \in \mathrm{C}_{b}(\hat{X})$ and that $\hat{\mathscr{A}}$ is an algebra. Clearly $\hat{\mathbb{1}}$ is the unit function in $\mathrm{C}_{b}(\hat{X})$ and $\hat{A}$ separates the points of $\hat{X}$ : if $\varphi_{i} \in \hat{X}$ satisfies $\varphi_{1}(f)=\varphi_{2}(f)$ for every $f \in \mathscr{A}$, then $\varphi_{1}=\varphi_{2}$ in $\mathscr{A}^{*}$. Applying Stone-Weierstrass Theorem, we conclude that $\hat{\mathscr{A}}$ is dense in $\mathrm{C}_{b}(\hat{X})$. By construction $\Gamma$ is a isomorphism between $\mathscr{A}$ and $\hat{\mathscr{A}}$, and $\iota^{*}$ is its inverse.

(c) Since $(\hat{X}, \hat{\tau})$ is compact and $\hat{\mathscr{A}}$ separates the points of $\hat{X}, \hat{\tau}$ is the initial topology of $\hat{\mathscr{A}}$. Thus a net $\hat{x}_{i}=\iota\left(x_{i}\right)$ in $\iota(X), i \in I$, converges to $\hat{x}=\iota(x)$ if and only if $\lim _{i \in I} \hat{f}\left(\hat{x}_{i}\right)=\hat{f}(\hat{x})$ for every $\hat{f} \in \hat{\mathscr{A}}$. By (2.79) and (2.80) the latter property is equivalent to $\lim _{i \in I} f\left(x_{i}\right)=f(x)$ for every $f \in \mathscr{A}$, so $\iota$ is a homeomorphism between $X$ endowed with the initial topology of $\mathscr{A}$ and $\iota(X)$.

(d) This is a general property for the push-forward of Radon measures through a continuous map: since $\mathfrak{m}$ is tight, we can find an increasing sequence of compact sets $K_{n} \subset X$ such that $\mathfrak{m}\left(X \backslash K_{n}\right) \leq$ 
$1 / n$. Since $\iota$ is continuous, $\hat{K}_{n}=\iota\left(K_{n}\right) \subset \iota(X)$ are compact in $\hat{X}$ and $\hat{\mathfrak{m}}\left(\hat{X} \backslash \hat{K}_{n}\right) \leq 1 / n$ so that $\hat{\mathfrak{m}}(\hat{X} \backslash \hat{K})=0$ where $\hat{K}=\cup_{n} \hat{K}_{n} \subset \iota(X)$.

(e) and (f) are immediate.

(g) Thanks to Lemma 2.31 the Gelfand isomorphism $\Gamma$ preserves the equivalence classes in the sense that for every $f_{1}, f_{2} \in \mathscr{A}$,

$$
f_{1} \sim_{\mathfrak{m}} f_{2} \Leftrightarrow \hat{f}_{1} \sim_{\hat{\mathfrak{m}}} \hat{f}_{2}
$$

so that

$$
\iota_{*}\left([f]_{\mathfrak{m}}\right)=[\hat{f}]_{\hat{\mathfrak{m}}} \text { for every } f \in \mathscr{A} .
$$

Since $\iota_{*}$ is a linear isometry and the equivalence classes of elements of $\mathscr{A}$ are dense in $L^{p}(X, \mathfrak{m})$ we conclude.

Remark 2.35 (A universal model). The compactification $\hat{\mathbb{X}}=(\hat{X}, \hat{\tau}, \hat{\mathrm{d}}, \hat{\mathfrak{m}})$ with the Gelfand algebra $\hat{\mathscr{A}}$ is a particular case of the case considered Example 2.11, where $B$ is the space $\overline{\mathscr{A}}, L=\overline{\mathscr{A}_{1}}$ and $\hat{X}$ is a weakly* compact subset of the dual ball of $B^{\prime}$ (in fact concentrated on its extremal set). It follows that any e.m.t.m. space has a measure-preserving isometric immersion in a space with the characteristics of Example 2.11.

The previous construction is also useful to quickly get a completion of an e.m.t.m. space. We start from the compactification $\hat{\mathbb{X}}=(\hat{X}, \hat{\tau}, \hat{\mathrm{d}}, \hat{\mathfrak{m}})$ of $(\mathbb{X}, \mathscr{A}(\mathbb{X}))$ obtained by the canonical algebra $\operatorname{Lip}_{b}(X, \mathrm{~d}, \mathfrak{m})$ and we set:

$$
\bar{X}:=\text { the } \hat{\mathrm{d}} \text {-closure of } \iota(X) \text { into } \hat{X} \text {. }
$$

We obviously define $\bar{\tau}, \overline{\mathrm{d}}$ and $\overline{\mathfrak{m}}$ respectively as the restrictions of $\hat{\tau}, \hat{\mathrm{d}}$, and $\hat{\mathfrak{m}}$ to $\bar{X}$.

Corollary 2.36 (Completion). The map $\iota: X \rightarrow \bar{X}$ is a measure preserving embedding of $\mathbb{X}$ into the e.m.t.m. space $\overline{\mathbb{X}}=(\bar{X}, \bar{\tau}, \overline{\mathrm{d}}, \overline{\mathrm{m}})$ such that

(C1) $(\bar{X}, \overline{\mathrm{d}})$ is complete,

(C2) $\iota$ is a homeomorphism of $X$ onto $\iota(X)$,

(C3) $\iota(X)$ is $\overline{\mathrm{d}}$-dense in $\bar{X}$,

(C4) every function $f \in \operatorname{Lip}_{b}(X, \tau, \mathrm{d})$ admits a unique extension to a function $\bar{f} \in \operatorname{Lip}_{b}(\bar{X}, \bar{\tau}, \overline{\mathrm{d}})$. The map $f \mapsto \bar{f}$ is an isomorphism of $\operatorname{Lip}_{b}(X, \tau, \mathrm{d})$ onto $\operatorname{Lip}_{b}(\bar{X}, \bar{\tau}, \overline{\mathrm{d}})$.

The space $\overline{\mathbb{X}}:=(\bar{X}, \hat{\tau}, \hat{\mathrm{d}}, \hat{\mathfrak{m}})$ is a completion of $\mathbb{X}$.

Notice that in the simple case when $\tau$ is the topology induced by the distance $\mathrm{d}$, the previous construction coincides with the usual completion of a metric space.

Remark 2.37. It is not difficult to check that if another e.m.t.m. space $\mathbb{X}^{\prime}$ satisfies the properties (C1)-(C4) of Corollary 2.36 then $\mathbb{X}^{\prime}$ is isomorphic to $\overline{\mathbb{X}}$, so that the completion of an e.m.t.m. space is unique up to isomorphisms. We may identify $X$ with the $\overline{\mathrm{d}}$-dense subset $\iota(X)$ in $\bar{X}$, so that for every function $f^{\prime}: \bar{X} \rightarrow \mathbb{R}, \iota^{*} f^{\prime}$ is just the restriction of $f^{\prime}$ to the $\overline{\mathfrak{m}}$-measurable set $X$ and $\mathfrak{m}$ can be considered as the restriction of $\overline{\mathfrak{m}}$ to $X$. Since $\overline{\mathfrak{m}}(\bar{X} \backslash X)=0$, we can identify $\overline{\mathfrak{m}}$ and $\mathfrak{m}$. Every function $f: X \rightarrow \mathbb{R}$ can be considered defined $\overline{\mathfrak{m}}$-a.e. and the trivial extension provides a realization of $\iota_{*}$ (which of course does not coincides with the extension $\bar{f}$ of $f \in \operatorname{Lip}_{b}(X, \tau, \mathrm{d}$ ) by continuity). 
We conclude this section with an example of application of the compactification trick to prove a useful approximation result.

Corollary 2.38. Let $\mathbb{X}=(X, \tau, \mathrm{d}, \mathfrak{m})$ be an e.m.t.m. space endowed with an adapted algebra $\mathscr{A}$. Then for every bounded upper semicontinuous function $g: X \rightarrow \mathbb{R}$

$$
\begin{aligned}
g(x) & =\inf \{h(x): h \in \mathscr{A}, h \geq g\}, \\
\int_{X} g \mathrm{~d} \mathfrak{m} & =\inf \left\{\int_{X} h(x) \mathrm{d} \mathfrak{m}: h \in \mathscr{A}, h \geq g\right\} .
\end{aligned}
$$

Proof. Let $\iota: X \rightarrow \hat{X}$ be the compactification of $\mathbb{X}$ induced by $\mathscr{A}$ and let $\hat{g}: \hat{X} \rightarrow \mathbb{R}$ be the upper semicontinuous envelope of $g$ to $\hat{X}$, i.e. the lowest upper semicontinuous function whose restriction to $X$ is greater or equal to $g$. We know that for every $\hat{x}=\iota(x) \in X$

$$
\hat{g}(\hat{x})=\inf _{U \in \mathscr{U}_{\hat{x}}} \sup \{g(y): y \in X, \iota(y) \in U\}=g(x)
$$

since $g$ is upper semicontinuous; here we use the fact that $\iota$ is an homeomorphism between $(X, \tau)$ and $(\iota(X), \hat{\tau})$, since $\mathscr{A}$ is adapted). Thanks to (the u.s.c. version of) (2.4) we deduce that

$$
\hat{g}(\hat{x})=\inf \left\{h(\hat{x}): h \in \mathrm{C}_{b}(\hat{X}, \hat{\tau}), h \geq \hat{g}\right\}
$$

and since $\hat{\mathscr{A}}=\Gamma(\mathscr{A})$ is uniformly dense in $\mathrm{C}_{b}(\hat{X}, \hat{\tau})$ we deduce the formula

$$
\hat{g}(\hat{x})=\inf \{\hat{h}(\hat{x}): h \in \mathscr{A}, h \geq g \text { in } X\}
$$

which (2.85). Since $H:=\left\{h \in \mathrm{C}_{b}(\hat{X}, \hat{\tau}), h \geq \hat{g}\right\}$ is a directed set by the order relation $h_{0} \prec h_{1} \Leftrightarrow$ $h_{0}(x) \leq h_{1}(x)$ for every $x \in \hat{X},(2.8)$ yields

$$
\int_{\hat{X}} \hat{g} \mathrm{~d} \hat{\mathfrak{m}}=\lim _{h \in H} \int_{\hat{X}} h \mathrm{~d} \hat{\mathfrak{m}}=\inf _{h \in H} \int_{\hat{X}} h \mathrm{~d} \hat{\mathfrak{m}} .
$$

Since $\Gamma(\mathscr{A})$ is uniformly dense in $\mathrm{C}_{b}(\hat{X}, \hat{\tau})$, every function $h \in H$ can be uniformly approximated from above by functions of the forms $\hat{f}=\Gamma(f)$ for $f \in \mathscr{A}, \Gamma(f) \geq h \geq \hat{g}$; we deduce that

$$
\int_{\hat{X}} \hat{g} \mathrm{~d} \hat{\mathfrak{m}}=\inf _{f \in \mathscr{A}, f \geq g} \int_{\hat{X}} \hat{f} \mathrm{~d} \hat{\mathfrak{m}}=\inf _{f \in \mathscr{A}, f \geq g} \int_{X} f \mathrm{~d} \mathfrak{m} .
$$

\subsection{Notes}

$\S$ 2.1: general references for measure theory are $[19,57]$; here we mainly followed the approach to Radon measures given by [57], trying to minimize the topological assumptions. The main points are the complete regularity of $(X, \tau)$ (which is almost needed for the standard formulation of the weak convergence of probability measures, see the Appendix of [57]) and the Radon property of the reference measure $\mathfrak{m}$. Complete regularity is in fact equivalent to the fact that continuous functions characterizes the topology of $X$ (see $\S$ A.2 in the Appendix) and it is also important for the formulation of the extended metric-topological spaces and for the compactification argument of $\S 2.7$.

$\S$ 2.2: here we followed very closely the presentation of [3, Section 3]. Extended metrics and the use of an auxiliary topology have already been considered in [9], under a slightly different set of compatibility conditions. 
$\S$ 2.4: the section just recalls the basic properties of the Kantorovich-Rubinstein distance, adapted to the extended setting, General references are the books [64, 7, 65]; notice that $\mathrm{K}_{\delta}$ is sometimes called KantorovichWasserstein distance of order 1 and denoted by $W_{1}$. The approximation result is quite similar to [3], where the distance of order 2 has been considered.

$\S$ 2.5: most of the result are classic for local slope (2.50). We adapted the same approach of [9] to the more refined (and stronger) local Lipschitz constant, which in the present setting also depends on the topology $\tau$. (2.51c) plays a crucial role in the locality property of the Cheeger energy and (2.51e) is quite useful to derive contraction estimate for its $L^{2}$ gradient flow. It is in fact possible to prove a more refined property, see [52].

$\S 2.6$ contains the main definition of algebras of functions compatible with the extended metric-topological setting. The basic requirements is that the algebra is sufficiently rich to recover the extended distance. We also collected a few results, mainly based on Bernstein polynomials [50], which will be quite useful to replace Lipschitz truncations with smoother polynomial maps preserving the algebraic structure.

$\S$ 2.7: measure-preserving isometric embeddings play an important role in the theory of metric-measure spaces, in particular when one studies their convergence (see [65, Chap. 27], [41]). Here we adapted this notion to the presence of the auxiliary topology $\tau$ and of the compatible algebra $\mathscr{A}$. Another typical application arises in regular representation of Dirichlet spaces (the so-called transfer method, [53, Chap. VI]); the idea of using the Gelfand transform to construct a suitable compactification is taken from [32], [33, Appendix A.4] and it is based on one of the possible construction of the Stone-Cech compactification of a completely regular topological space.

\section{Continuous curves and nonparametric arcs}

This section mostly contains classic material on the topology of space of curves, adapted to the extended metric-topological setting. Its main goal is to construct a useful setting to deal with Radon measures on (non parametric) rectifiable curves. Differently from other approaches (see e.g. [55, 46, 2]) we first study class of equivalent curves (up to reparametrizations) without assuming their rectifiability. In $\S 3.1$ we study the natural e.m.t. structure on $\mathrm{C}([a, b] ;(X, \tau))$, and in $\S 3.2$ we consider the natural quotient space of (continuous) arcs $\mathrm{A}(X, \tau)$, which behaves quite well with respect to topology and distance. The last part 3.3 is focused in d-rectifiable $\operatorname{arcs} \operatorname{RA}(X, \mathrm{~d})$, considered as a natural Borel subset of $\mathrm{A}(X, \tau)$. Here we have the arc-length reparametrization at our disposal, and we study measurability properties of important operations, like evaluations, integrals, length, and reparametrizations. We will also state a useful compactness result, which is a natural generalization of Arzelà-Ascoli Theorem. Theorem 3.13 will collect most of the main properties we will use in the next chapters.

\subsection{Continuous curves}

Let $(X, \tau)$ be a completely regular Hausdorff space. We will denote by $\mathrm{C}([a, b] ;(X, \tau))$ the set of $\tau$-continuous curves $\gamma:[a, b] \rightarrow X$ endowed with the compact-open topology $\tau_{\mathrm{C}}$ (we will simply write $\mathrm{C}([a, b] ; X)$, when the topology $\tau$ will be clear from the context). By definition, a subbasis generating $\tau_{C}$ is given by the collection of sets

$$
S(K, V):=\{\gamma \in \mathrm{C}([a, b] ; X): \gamma(K) \subset V\}, \quad K \subset[a, b] \text { compact, } V \text { open in } X .
$$

Remark 3.1. Thanks to the particular structure of the domain $[a, b]$, we can also consider an equivalent basis associated to partitions $\mathcal{P}=\left\{a=t_{0}<t_{1}<\cdots<t_{J}=b\right\}$ :

$$
\cap_{j=1}^{J}\left\{\gamma \in \mathrm{C}([a, b] ; X): \gamma\left(\left[t_{j-1}, t_{j}\right]\right) \subset W_{j}\right\}, \quad t_{j} \in \mathcal{P}, W_{j} \text { open in } X .
$$


It is also not restrictive to consider partitions $\mathcal{P}$ induced by rational points $\left.t_{j} \in \mathbb{Q} \cap\right] a, b[, j=1, \cdots, J$. It is sufficient to show that if $\gamma_{0}$ belongs to an open set $U=\cap_{h=1}^{H} S\left(K_{h}, V_{h}\right)$ arising from the finite intersection of elements of the subbasis (3.1), we can also find a set $U^{\prime}$ of the form (3.2) such that $\gamma_{0} \in U^{\prime} \subset U$. To this aim, it is not restrictive to add to the collection $\left\{K_{h}, V_{h}\right\}_{h=1}^{H}$ the couple $K_{0}=[a, b], V_{0}=X$; we can cover each $K_{h}$ with a finite number of intervals $I_{h, k}=\left[\alpha_{h, k}, \beta_{h, k}\right]$, $1 \leq k \leq k(h)$, such that $\gamma_{0}\left(I_{h, k}\right) \subset V_{h}$. We can then take the partition $\mathcal{P}$ of $[a, b]$ containing all the extrema of $I_{h, k}$ (notice that $a$ and $b$ are included). If $t_{j}, j \geq 1$, is a point of the partition $\mathcal{P}$, we set $W_{j}:=\cap\left\{V_{h}: \exists k \in\{1, \cdots, k(h)\},\left[t_{j-1}, t_{j}\right] \subset I_{h, k}\right\}$.

Let us recall a few simple and useful properties of the compact-open topology [54, § 46]:

(CO1) If the topology $\tau$ is induced by a distance $\delta$, then the topology $\tau_{\mathrm{C}}$ is induced by the uniform distance $\mathrm{d}_{\delta}\left(\gamma, \gamma^{\prime}\right):=\sup _{t \in[a, b]} \delta\left(\gamma(t), \gamma^{\prime}(t)\right)$ and convergence w.r.t. the compact-open topology coincides with the uniform convergence w.r.t. $\delta$. If moreover $\tau$ is separable then also $\tau_{\mathrm{C}}$ is separable.

(CO2) The evaluation map e : $[a, b] \times \mathrm{C}([a, b] ; X) \rightarrow X, \mathrm{e}(t, \gamma):=\gamma(t)$, is continuous.

(CO3) If $f: X \rightarrow Y$ is a continuous function with values in a Hausdorff space $\left(Y, \tau_{Y}\right)$, the composition map $\gamma \mapsto f \circ \gamma$ is continuous from $\mathrm{C}([a, b] ; X)$ to $\mathrm{C}([a, b] ; Y)$.

(CO4) If $\sigma:[0,1] \rightarrow[0,1]$ is nondecreasing and continuous the map $\gamma \mapsto \gamma \circ \sigma$ is continuous from $\mathrm{C}([a, b] ; X)$ to $\mathrm{C}([a, b] ; X)$.

(CO5) If $\tau$ is Polish, then $\tau_{\mathrm{C}}$ is Polish.

The proof of the first two properties can be found, e.g., in [54, Thm. 46.8, 46.10]; in the case when $\tau$ is metrizable and separable, the separability of $\tau_{\mathrm{C}}$ follows by [29, 4.2.18].

In order to prove (CO3) it is sufficient to show that the inverse image of an arbitrary element $S(K, W)$ (as in (3.1)) of the subbasis generating the compact-open topology of $\mathrm{C}([a, b] ; Y)$ is an element of the corresponding subbasis of the topology of $\mathrm{C}([a, b] ; X)$. In fact, $f \circ \gamma \in S(K, W)$ if and only if $\gamma \in S\left(K, f^{-1}(W)\right)$. (CO4) can be proved by a similar argument: if $\gamma \circ \sigma \in S(K, V)$ if and only if $\gamma \in S(\sigma(K), V)$. Finally (CO5) is a consequence of (CO1).

We will denote by e $(\gamma)$ the image $\gamma([a, b])$ in $X$.

(Semi)distances on $\mathrm{C}([a, b] ; X)$

An extended semidistance $\delta: X \times X \rightarrow[0, \infty]$ induces an extended semidistance $\delta_{\mathrm{C}}$ in $\mathrm{C}([a, b] ; X)$ by

$$
\delta_{\mathrm{C}}\left(\gamma_{1}, \gamma_{2}\right):=\sup _{t \in[a, b]} \delta\left(\gamma_{1}(t), \gamma_{2}(t)\right)
$$

We have

Proposition 3.2. If $(X, \tau, \mathrm{d})$ is an extended metric-topological space, than also $\left(\mathrm{C}([a, b] ;(X, \tau)), \tau_{\mathrm{C}}, \mathrm{d}_{\mathrm{C}}\right)$ is an extended metric-topological space.

If moreover $\tau^{\prime}$ is an auxiliary topology for $(X, \tau, \mathrm{d})$ according to Definition 2.6, then $\tau_{\mathrm{C}}^{\prime}$ is an auxiliary topology for $\left(\mathrm{C}([a, b] ;(X, \tau)), \tau_{\mathrm{C}}, \mathrm{d}_{\mathrm{C}}\right)$ as well. 
Proof. Notice that for every $f \in \operatorname{Lip}_{b, 1}(X, \tau, \mathrm{d})$ and $t \in[a, b]$ the map $\mathrm{f}_{t}:=f \circ \mathrm{e}_{t}$ belongs to $\operatorname{Lip}_{b, 1}\left(\mathrm{C}([a, b] ; X), \tau_{\mathrm{C}}, \mathrm{d}_{\mathrm{C}}\right)$ and it is easy to check by $(2.17)$ that

$$
\mathrm{d}_{\mathrm{C}}\left(\gamma_{1}, \gamma_{2}\right)=\sup \left\{\left|\mathrm{f}_{t}\left(\gamma_{1}\right)-\mathrm{f}_{t}\left(\gamma_{2}\right)\right|: f \in \operatorname{Lip}_{b, 1}(X, \tau, \mathrm{d}), t \in[a, b]\right\} .
$$

For every finite collection $\mathcal{K}:=\left\{K_{h}\right\}_{h=1}^{H}$ of compact subsets of $[a, b]$ and for every finite collection $\mathcal{F}:=\left(f_{h}\right)_{h=1}^{H}$ in $\operatorname{Lip}_{b}(X, \tau, \mathrm{d})$ let us consider the function $F=F_{\mathcal{K}, \mathcal{F}}$ defined by

$$
F(\gamma):=\max _{1 \leq h \leq H} \max _{t \in K_{h}} f_{h}(\gamma(t))
$$

It is easy to check that $F \in \operatorname{Lip}_{b}\left(\mathrm{C}([a, b] ; X), \tau_{\mathrm{C}}, \mathrm{d}_{\mathrm{C}}\right)$; we want to show that this family of functions separates points from closed sets. To this aim, we fix a closed set $\mathrm{F} \subset \mathrm{C}([a, b] ; X)$ and a curve $\gamma_{0} \in \mathrm{C}([a, b] ; X) \backslash \mathrm{F}$.

By the definition of compact-open topology, we can find a collection $\mathcal{K}=\left\{K_{h}\right\}_{h=1}^{H}$ of compact sets of $[a, b]$ and open sets $U_{h} \subset X$ such that the open set $\mathrm{U}=\left\{\gamma: \gamma\left(K_{h}\right) \subset U_{h}, 1 \leq h \leq H\right\}$ is included in $\mathrm{C}([a, b] ; X) \backslash \mathrm{F}$ and contains $\gamma_{0}$. By (2.2) and the compactness we can find nonnegative functions $\mathcal{F}=\left(f_{h}\right)_{h=1}^{H} \subset \operatorname{Lip}_{b}(X, \tau, \mathrm{d})$ such that $f_{h} \equiv 1$ on $X \backslash U_{h}$ and $\left.f_{h}\right|_{\gamma_{0}\left(K_{h}\right)} \equiv 0$. It follows that $F=F_{\mathcal{K}, \mathcal{F}}$ satisfies $F\left(\gamma_{0}\right)=0$ and $F(\gamma) \geq 1$ for every $\gamma$ in the complement of U, in particular in F.

Let us eventually check the last statement, by checking that $\tau_{\mathrm{C}}^{\prime}$ satisfies properties $(\mathrm{A} 1,2,3)$ of Definition 2.6. (A1,2) are obvious. Concerning (A3) we select a sequence $f_{n} \in \operatorname{Lip}_{b}\left(X, \tau^{\prime}, \mathrm{d}\right)$ satisfying (2.28) and the countable collection of maps $\mathcal{F}:=\left(\mathrm{f}_{n, t}\right)_{n \in \mathbb{N}, t \in[a, b] \cap \mathbb{Q}}, \mathrm{f}_{n, t}:=f_{n} \circ \mathrm{e}_{t}$. It is clear that for every $\gamma_{1}, \gamma_{2} \in \mathrm{C}([a, b] ; X)$ and every $n \in \mathbb{N}$

$$
\sup _{t \in[a, b]}\left|\mathrm{f}_{n, t}\left(\gamma_{1}\right)-\mathrm{f}_{n, t}\left(\gamma_{2}\right)\right|=\sup _{t \in[a, b] \cap \mathbb{Q}}\left|\mathrm{f}_{n, t}\left(\gamma_{1}\right)-\mathrm{f}_{n, t}\left(\gamma_{2}\right)\right|
$$

(2.28) then yields

$$
\mathrm{d}_{\mathrm{C}}\left(\gamma_{1}, \gamma_{2}\right)=\sup _{\mathbf{f} \in \mathcal{F}}\left|\mathrm{f}\left(\gamma_{1}\right)-\mathbf{f}\left(\gamma_{2}\right)\right| .
$$

We can state a useful compactness criterium, which is the natural extension of Arzelà-Ascoli Theorem to extended metric-topological structures.

Proposition 3.3. Let us suppose that $(X, \tau)$ is compact and let $\Gamma \subset \mathrm{C}([a, b] ; X)$ be $\mathrm{d}$-equicontinuous, i.e. there exists $\omega:[0, \infty) \rightarrow[0, \infty)$ concave, nondecreasing and continuous with $\omega(0)=0$ such that

$$
\mathrm{d}(\gamma(r), \gamma(s)) \leq \omega(|r-s|) \quad \text { for every } r, s \in[a, b], \gamma \in \Gamma .
$$

Then $\Gamma$ is relatively compact with respect to $\tau_{\mathrm{C}}$.

Proof. Let $\gamma_{i}, i \in I$, be a net in $\Gamma$. Since $X$ is compact, we can find a subnet $h: J \rightarrow I$ and a limit curve $\gamma:[a, b] \rightarrow X$ such that $\lim _{j \in J} \gamma_{h(j)}=\gamma$ with respect to the topology of pointwise convergence. Passing to the limit in (3.6) we immediately see that

$$
\mathrm{d}(\gamma(r), \gamma(s)) \leq \liminf _{j \in J} \mathrm{~d}\left(\gamma_{h(j)}(r), \gamma_{h(j)}(s)\right) \leq \omega(|r-s|) \quad \text { for every } r, s \in[a, b],
$$

so that $\gamma \in \mathrm{C}([a, b] ;(X, \mathrm{~d}))$.

Let us now consider a neighborhood $\mathcal{U}=\cap_{h=1}^{H} S\left(K_{h}, V_{h}\right)$ of $\gamma$ : we will show that there exists $j_{0} \in J$ such that $\gamma_{h(j)} \in \mathcal{U}$ for every $j \succeq j_{0}$. Let us consider functions $f_{h} \in \operatorname{Lip}_{b}(X, \tau, \mathrm{d})$ 
satisfying $f_{h} \equiv 0$ on $\gamma\left(K_{h}\right)$ and $f_{h} \equiv 1$ on $X \backslash V_{h}$ as in the previous Lemma and let $L>0$ be the maximum of the Lipschitz constants of $f_{h}$. We call $\boldsymbol{f}_{j}:[a, b] \rightarrow \mathbb{R}^{H}$ the family of curves $\boldsymbol{f}_{j}(t):=\left(f_{1}\left(\gamma_{h(j)}(t)\right), f_{2}\left(\gamma_{h(j)}(t)\right), \cdot f_{H}\left(\gamma_{h(j)}(t)\right)\right.$ indexed by $j \in J$. Since $f_{h}$ are continuous, we have

$$
\lim _{j \in J} \boldsymbol{f}_{j}(t)=\boldsymbol{f}(t) \quad \text { pointwise, } \quad \boldsymbol{f}(t):=\left(f_{1}(\gamma(t)), f_{2}(\gamma(t)), \cdot, f_{H}(\gamma(t)) .\right.
$$

On the other hand, we have

$$
\left|\boldsymbol{f}_{j}(r)-\boldsymbol{f}_{j}(s)\right| \leq H L \omega(|r-s|)
$$

so that Ascoli-Arzelà theorem (in $\left.\mathrm{C}\left([a, b] ; \mathbb{R}^{H}\right)\right)$ yields

$$
\lim _{j \in J} \sup _{t \in[a, b]}\left|\boldsymbol{f}_{j}(t)-\boldsymbol{f}(t)\right|=0 .
$$

Choosing $j_{0} \in J$ so that $\sup _{t \in[a, b]}\left|\boldsymbol{f}_{j}(t)-\boldsymbol{f}(t)\right| \leq 1 / 2$ for every $j \succeq j_{0}$, since $f_{h}(\gamma(t)) \equiv 0$ whenever $t \in K_{h}$ we deduce that $f_{h}\left(\gamma_{j}(t)\right) \leq 1 / 2$ whenever $t \in K_{h}$ so that $\gamma_{j}\left(K_{h}\right) \subset V_{h}$.

\subsection{Arcs}

Let us denote by $\Sigma$ the set of continuous, nondecreasing and surjective map $\sigma:[0,1] \rightarrow[0,1]$ and by $\Sigma^{\prime}$ the subset of $\Sigma$ of the invertible maps, thus increasing homeomorphisms of $[0,1]$. We also set

$$
\Sigma_{2}:=\{\sigma \in \Sigma:|\sigma(r)-\sigma(s)| \leq 2|r-s|\}
$$

On $\mathrm{C}([0,1] ;(X, \tau))$ we introduce the symmetric and reflexive relation

$$
\gamma_{1} \sim \gamma_{2} \text { if } \exists \sigma_{i} \in \Sigma: \gamma_{1} \circ \sigma_{1}=\gamma_{2} \circ \sigma_{2}
$$

Notice that $\gamma \sim \gamma \circ \sigma$ for every $\sigma \in \Sigma$. It is also not difficult to check that if a map $\gamma:[0,1] \rightarrow X$ satisfies $\gamma \circ \sigma \in \mathrm{C}([0,1] ;(X, \tau))$ for some $\sigma \in \Sigma$ then $\gamma \in \mathrm{C}([0,1] ;(X, \tau))$. In fact, if $A \subset X$ is a closed set, then $B:=(\gamma \circ \sigma)^{-1}(A)=\sigma^{-1}\left(\gamma^{-1}(A)\right)$ is closed in $[0,1]$ and therefore compact. Since $\sigma$ is surjective, for every $Z \subset[0,1]$ we have $\sigma\left(\sigma^{-1}(Z)\right)=Z$ so that $\gamma^{-1}(A)=\sigma(B)$ is closed since it is the continuous image of a compact set.

We want to show that $\sim$ also satisfies the transitive property, so that it is an equivalence relation.

If $\delta: X \times X \rightarrow[0,+\infty]$ is a $\tau$-l.s.c. extended semidistance we also introduce

$$
\delta_{\mathrm{A}}\left(\gamma_{1}, \gamma_{2}\right):=\inf _{\sigma_{i} \in \Sigma} \delta_{\mathrm{C}}\left(\gamma_{1} \circ \sigma_{1}, \gamma_{2} \circ \sigma_{2}\right) \quad \text { for every } \gamma_{i} \in \mathrm{C}([0,1] ;(X, \tau))
$$

and the set

$$
\mathrm{C}([0,1] ;(X, \tau, \delta)):=\left\{\gamma \in \mathrm{C}([0,1] ;(X, \tau)): \lim _{s \rightarrow t} \delta(\gamma(s), \gamma(t))=0 \quad \text { for every } t \in[0,1]\right\} .
$$

It is not difficult to check that

$$
\gamma \in \mathrm{C}([0,1] ;(X, \tau, \delta)) \quad \Rightarrow \quad \lim _{r \downarrow 0} \sup _{|t-s| \leq r} \delta(\gamma(s), \gamma(t))=0 ;
$$

in the case $\delta=\mathrm{d}$ we have

$$
\mathrm{C}([0,1] ;(X, \tau, \mathrm{d}))=\mathrm{C}([0,1] ;(X, \mathrm{~d})) .
$$


Theorem 3.4 (Reparametrizations and semidistances). Let $\delta: X \times X \rightarrow[0,+\infty]$ be a $\tau$-l.s.c. extended semidistance and let $\gamma_{i} \in \mathrm{C}([0,1] ;(X, \tau, \delta)), i=1,2,3$. We have

$$
\begin{aligned}
\delta_{\mathrm{A}}\left(\gamma_{1}, \gamma_{2}\right) & =\inf _{\sigma \in \Sigma^{\prime}} \delta_{\mathrm{C}}\left(\gamma_{1}, \gamma_{2} \circ \sigma\right) \\
& =\min _{\sigma_{i} \in \Sigma_{2}} \delta_{\mathrm{C}}\left(\gamma_{1} \circ \sigma_{1}, \gamma_{2} \circ \sigma_{2}\right) \\
& =\inf _{\gamma_{i}^{\prime} \sim \gamma_{i}} \delta_{\mathrm{C}}\left(\gamma_{1}^{\prime}, \gamma_{2}^{\prime}\right) .
\end{aligned}
$$

In particular $\delta_{\mathrm{A}}$ satisfies the triangle inequality

$$
\delta_{\mathrm{A}}\left(\gamma_{1}, \gamma_{3}\right) \leq \delta_{\mathrm{A}}\left(\gamma_{1}, \gamma_{2}\right)+\delta_{\mathrm{A}}\left(\gamma_{2}, \gamma_{3}\right)
$$

Proof. If $\sigma \in \Sigma$ we will still denote by $\sigma$ its extension to $\mathbb{R}$ defined by the map $r \mapsto \sigma(0 \vee r \wedge 1)$. We introduce the $(1+\varepsilon)$-Lipschitz map $j_{\sigma, \varepsilon}: \mathbb{R} \rightarrow \mathbb{R}$ defined by

$$
s=j_{\sigma, \varepsilon}(r) \quad \Leftrightarrow \quad s+\varepsilon \sigma(s)=(1+\varepsilon) r
$$

and the maps $\hat{\sigma}_{\varepsilon}, \sigma_{\varepsilon}: \mathbb{R} \rightarrow \mathbb{R}$

$$
\begin{aligned}
& \hat{\sigma}_{\varepsilon}(r):=(1+\varepsilon)^{-1}(\varepsilon r+\sigma(r)), \quad\left|\hat{\sigma}_{\varepsilon}(r)-\sigma(r)\right| \leq \frac{\varepsilon}{1+\varepsilon}|r-\sigma(r)| \leq \varepsilon, \\
& \sigma_{\varepsilon}(r):=\varepsilon^{-1}\left((1+\varepsilon) r-j_{\sigma, \varepsilon}(r)\right)=\sigma\left(j_{\sigma, \varepsilon}(r)\right) .
\end{aligned}
$$

Notice that the restrictions to $[0,1]$ of the maps $\hat{\sigma}_{\varepsilon}, j_{\sigma, \varepsilon}, \sigma_{\varepsilon}$ operate in $[0,1]$ and $\hat{\sigma}_{\varepsilon} \in \Sigma^{\prime}, j_{\sigma, \varepsilon}, \sigma_{\varepsilon} \in \Sigma$, $\sigma_{\varepsilon}$ is $(1+1 / \varepsilon)$-Lipschitz. We also denote by $\omega_{\gamma}$ the $\delta$-modulus of continuity of $\gamma \in \mathrm{C}([0,1] ;(X, \tau, \delta))$, i.e.

$$
\omega_{\gamma}(r):=\sup \{\delta(\gamma(s), \gamma(t)): s, t \in[0,1],|s-t| \leq r\},
$$

observing that $\lim _{r \downarrow 0} \omega_{\gamma_{i}}(r)=0$ in the case of the curves $\gamma_{1}, \gamma_{2}$ considered by the Lemma.

In order to prove (3.16a), we observe that that $\delta_{\mathrm{C}}$ is invariant w.r.t. composition with arbitrary $\sigma \in \Sigma$

$$
\delta_{\mathrm{C}}\left(\gamma_{1}, \gamma_{2}\right)=\delta_{\mathrm{C}}\left(\gamma_{1} \circ \sigma, \gamma_{2} \circ \sigma\right) \text { for every } \gamma_{i} \in \mathrm{C}([0,1] ; X), \sigma \in \Sigma,
$$

and every $\sigma \in \Sigma$ can be uniformly approximated by the increasing homeomorphisms $\hat{\sigma}_{\varepsilon} \in \Sigma^{\prime}$; we easily get

$$
\delta_{\mathrm{C}}\left(\gamma \circ \sigma, \gamma \circ \hat{\sigma}_{\varepsilon}\right) \leq \omega_{\gamma}\left(\sup _{r \in[0,1]}\left|\sigma(r)-\hat{\sigma}_{\varepsilon}(r)\right|\right) \stackrel{(3.19)}{\leq} \omega_{\gamma}(\varepsilon),
$$

so that the triangle inequality for $\delta_{\mathrm{C}}$ yields

$$
\begin{aligned}
\delta_{\mathrm{C}}\left(\gamma_{1} \circ \sigma_{1}, \gamma_{2} \circ \sigma_{2}\right) & \geq \delta_{\mathrm{C}}\left(\gamma_{1} \circ \hat{\sigma}_{1, \varepsilon}, \gamma_{2} \circ \sigma_{2}\right)-\omega_{\gamma_{1}}(\varepsilon) \geq \delta_{\mathrm{C}}\left(\gamma_{1}, \gamma_{2} \circ \sigma_{2}^{\prime}\right)-\omega_{\gamma_{1}}(\varepsilon) \\
& \geq \delta_{\mathrm{C}}\left(\gamma_{1}, \gamma_{2} \circ \hat{\sigma}_{2, \varepsilon}^{\prime}\right)-\omega_{\gamma_{1}}(\varepsilon)-\omega_{\gamma_{2}}(\varepsilon) \\
& \geq \inf _{\sigma \in \Sigma^{\prime}} \delta_{\mathrm{C}}\left(\gamma_{1}, \gamma_{2} \circ \sigma\right)-\omega_{\gamma_{1}}(\varepsilon)-\omega_{\gamma_{2}}(\varepsilon)
\end{aligned}
$$

where $\sigma_{2}^{\prime}:=\sigma_{2} \circ\left(\hat{\sigma}_{1, \varepsilon}\right)^{-1}$ and $\hat{\sigma}_{2, \varepsilon}^{\prime}$ is obtained by $\left(\sigma_{2}^{\prime}\right)_{\varepsilon}$ as in (3.19). Taking the infimum w.r.t. $\sigma_{1}, \sigma_{2} \in$ $\Sigma$ and passing to the limit as $\varepsilon \downarrow 0$ we obtain

$$
\delta_{\mathrm{A}}\left(\gamma_{1}, \gamma_{2}\right) \geq \inf _{\sigma \in \Sigma^{\prime}} \delta_{\mathrm{C}}\left(\gamma_{1}, \gamma_{2} \circ \sigma\right) .
$$


Since the opposite inequality is obvious, we get (3.16a).

The triangle inequality is an immediate consequence of (3.16a): if $\gamma_{1}, \gamma_{2}, \gamma_{3} \in \mathrm{C}([0,1] ;(X, \tau, \delta))$ and $\sigma_{1}, \sigma_{3} \in \Sigma^{\prime}$ we have

$$
\delta_{\mathrm{A}}\left(\gamma_{1}, \gamma_{3}\right) \leq \delta_{\mathrm{C}}\left(\gamma_{1} \circ \sigma_{1}, \gamma_{3} \circ \sigma_{3}\right) \leq \delta_{\mathrm{C}}\left(\gamma_{1} \circ \sigma_{1}, \gamma_{2}\right)+\delta_{\mathrm{C}}\left(\gamma_{2}, \gamma_{3} \circ \sigma_{3}\right) ;
$$

taking the infimum w.r.t. $\sigma_{1}, \sigma_{3}$ we obtain (3.17).

Let us now prove (3.16b), i.e. the infimum in (3.12) is attained by a couple $\varrho_{i} \in \Sigma$ given by 2-Lipschitz maps. We observe that

$$
\delta_{\mathrm{C}}\left(\gamma_{1}, \gamma_{2} \circ \varrho\right) \stackrel{(3.22)}{=} \delta_{\mathrm{C}}\left(\gamma_{1} \circ j_{\varrho, \varepsilon}, \gamma_{2} \circ \varrho \circ j_{\varrho, \varepsilon}\right) \stackrel{(3.20)}{=} \delta_{\mathrm{C}}\left(\gamma_{1} \circ j_{\varrho, \varepsilon}, \gamma_{2} \circ \varrho_{\varepsilon}\right)
$$

and $j_{\varrho, \varepsilon}$ is $(1+\varepsilon)$-Lipschitz, $\varrho_{\varepsilon}$ is $\left(1+\varepsilon^{-1}\right)$-Lipschitz. Choosing $\varepsilon=1$ we deduce that the infimum in (3.12) can be restricted to $\Sigma_{2}$. Since $\Sigma_{2}$ is compact w.r.t. uniform convergence, $\gamma_{i}$ are continuous and $\delta_{\mathrm{C}}$ is lower semicontinuous w.r.t. $\tau_{\mathrm{C}}$, we obtain $(3.16 \mathrm{~b})$.

Let us eventually consider (3.16c); since $\inf _{\gamma_{i}^{\prime} \sim \gamma_{i}} \delta_{\mathrm{C}}\left(\gamma_{1}^{\prime}, \gamma_{2}^{\prime}\right) \leq \delta_{\mathrm{A}}\left(\gamma_{1}, \gamma_{2}\right)$ it is sufficient to prove the opposite inequality. By (3.17) we have

$$
\delta_{\mathrm{C}}\left(\gamma_{1}^{\prime}, \gamma_{2}^{\prime}\right) \geq \delta_{\mathrm{A}}\left(\gamma_{1}^{\prime}, \gamma_{2}^{\prime}\right) \geq-\delta_{\mathrm{A}}\left(\gamma_{1}^{\prime}, \gamma_{1}\right)+\delta_{\mathrm{A}}\left(\gamma_{1}, \gamma_{2}\right)-\delta_{\mathrm{A}}\left(\gamma_{2}, \gamma_{2}^{\prime}\right)=\delta_{\mathrm{A}}\left(\gamma_{1}, \gamma_{2}\right)
$$

Corollary 3.5. The relation $\sim$ satisfies the transitive property and it is an equivalent relation. Moreover

(a) The space $\mathrm{A}(X, \tau):=\mathrm{C}([0,1] ;(X, \tau)) / \sim$ endowed with the quotient topology $\tau_{\mathrm{A}}$ is an Hausdorff space. We will denote by $[\gamma]$ the corresponding equivalence class associated to $\gamma \in \mathrm{C}([0,1] ;(X, \tau))$ and by $\mathfrak{q}: \mathrm{C}([0,1] ;(X, \tau)) \rightarrow \mathrm{A}(X, \tau)$ the quotient map $\gamma \mapsto[\gamma]$.

(b) If $\delta$ is a $\tau$-continuous semidistance, then $\delta_{\mathrm{A}}$ is a $\tau_{\mathrm{A}}$ continuous semidistance (considered as a function between equivalence classes of curves).

(c) If the topology $\tau$ is induced by the distance $\delta$ then the quotient topology $\tau_{\mathrm{A}}$ is induced by $\delta_{\mathrm{A}}$ (considered as a distance between equivalence classes of curves).

(d) If $(X, \tau)$ is a Polish space, then $\left(\mathrm{A}(X, \tau), \tau_{\mathrm{A}}\right)$ is a Souslin metrizable space.

Proof. (a) Let us first prove the transitivity of $\sim$. Let $\gamma_{i} \in \mathrm{C}([0,1] ; X), i=1,2,3$, such that $\gamma_{1} \sim \gamma_{2}$ and $\gamma_{2} \sim \gamma_{3}$ and let $K:=\cup_{i=1}^{3} \gamma_{i}([0,1])$. $K$ is a compact and separable set; applying Remark 2.5 we can find a bounded and continuous semidistance $\delta$ whose restriction to $K \times K$ induces the $\tau$-topology.

By the very definition (3.12) of $\delta_{\mathrm{A}}$ we get $\delta_{\mathrm{A}}\left(\gamma_{1}, \gamma_{2}\right)=0$ and $\delta_{\mathrm{A}}\left(\gamma_{2}, \gamma_{3}\right)=0$, so that (3.17) yields $\delta_{\mathrm{A}}\left(\gamma_{1}, \gamma_{3}\right)=0$ and $(3.16 \mathrm{~b})$ yields $\gamma_{1} \sim \gamma_{3}$.

Let us now show that $\left(\mathrm{A}(X), \tau_{\mathrm{A}}\right)$ is Hausdorff. We fix two curves $\gamma_{1}, \gamma_{2} \in \mathrm{C}([0,1] ; X)$ such that $\left[\gamma_{1}\right] \neq\left[\gamma_{2}\right]$, we consider the compact and separable subspace $K:=\gamma_{1}([0,1]) \cup \gamma_{2}([0,1])$, and a bounded $\tau$-continuous semidistance $\delta$, generated as in Remark 2.5, whose restriction to $K$ induces the $\tau$-topology.

We notice that the maps $\gamma \mapsto \delta_{\mathrm{C}}\left(\gamma_{i}, \gamma\right)=\sup _{t \in[0,1]} \delta\left(\gamma_{i}(t), \gamma(t)\right)$ are continuous in $\mathrm{C}([0,1] ; X)$; since the composition maps $\gamma \mapsto \gamma \circ \varrho, \varrho \in \Sigma^{\prime}$, are continuous, we deduce that the maps $\gamma \mapsto$ $\delta_{\mathrm{A}}\left(\left[\gamma_{i}\right],[\gamma]\right)=\inf _{\varrho \in \Sigma^{\prime}} \delta_{\mathrm{C}}\left(\gamma_{i}, \gamma \circ \varrho\right)$ are upper semicontinuous from $\mathrm{C}([0,1] ; X)$ to $\mathbb{R}$. By the above discussion, if $\left[\gamma_{1}\right] \neq\left[\gamma_{2}\right]$ we get $\delta_{\mathrm{A}}\left(\gamma_{1}, \gamma_{2}\right)=\delta>0$ so that the open sets $U_{i}:=\{\gamma \in \mathrm{C}([0,1] ; X)$ : $\left.\delta_{\mathrm{A}}\left(\gamma_{i}, \gamma\right)<\delta / 2\right\}$ are disjoint (by the triangle inequality) saturated open neighborhoods of $\gamma_{i}$. 
(b) Since $\delta_{\mathrm{A}}$ is continuous w.r.t. $\tau_{\mathrm{C}}$ and it is invariant w.r.t. the equivalence relation, it is clear that $\delta_{\mathrm{A}}$ is continuous w.r.t. $\tau_{\mathrm{A}}$.

(c) If $\gamma_{0} \in \mathrm{C}([0,1] ; X)$ every $\tau_{\mathrm{A}}$ open neighborhood $U_{0}$ of $\left[\gamma_{0}\right]$ in $\mathrm{A}(X)$ correspond to a saturated open set $U$ of $\mathrm{C}([0,1] ; X)$ containing $\gamma_{0}$. In particular, there exists $\varepsilon>0$ such that $U$ contains all the curves $\gamma \in \mathrm{C}([0,1] ; X)$ with $\delta_{\mathrm{C}}\left(\gamma, \gamma_{0}\right)<\varepsilon$ and all the curves of the form $\gamma \circ \sigma, \gamma_{0} \circ \sigma_{0}$ for arbitrary $\sigma, \sigma_{0} \in \Sigma$. It follows that $U_{0}$ contains $\left\{[\gamma]: \delta_{\mathrm{A}}\left([\gamma],\left[\gamma_{0}\right]\right)<\varepsilon\right\}$.

(d) The Souslin property is an immediate consequence of the fact that $\left(\mathrm{A}(X, \tau), \tau_{\mathrm{A}}\right)$ is obtained as a quotient space of the Polish space $\left(\mathrm{C}([0,1] ;(X, \tau)), \tau_{\mathrm{C}}\right)$. The metrizability follows by the previous claim.

The image of an arc $[\gamma]$ is independent of the parametrization and it will still be denoted by $\mathrm{e}([\gamma])$; we will also set $\mathrm{e}_{i}([\gamma]):=\mathrm{e}_{i}(\gamma), i=0,1$. Every function $f: \mathrm{A}(X, \tau) \rightarrow Y$ is associated to a "lifted" function $\tilde{f}: \mathrm{C}([0,1] ;(X, \tau)) \rightarrow Y, \tilde{f}(\gamma):=f([\gamma])$, whose values are invariant by reparametrizations of $\gamma . f$ is continuous (w.r.t. the quotient topology $\tau_{\mathrm{A}}$ ) if and only if $\tilde{f}$ is continuous (w.r.t. the compactopen topology $\left.\tau_{\mathrm{C}}\right)$.

Let us now consider the case of an extended metric-topological space. We denote by $\mathrm{A}(X, \mathrm{~d})$ the subset of arcs admitting an equivalent $d$-continuous parametrization (or, equivalently, whose equivalent parametrizations are d-continuous). Notice that $\mathrm{A}(X, \mathrm{~d}) \subset \mathrm{A}(X, \tau)$.

Proposition 3.6. Let $(X, \tau, \mathrm{d})$ be an extended metric-topological space. $\left(\mathrm{A}(X, \mathrm{~d}), \tau_{\mathrm{A}}, \mathrm{d}_{\mathrm{A}}\right)$ is an extended metric-topological space.

Proof. The fact that $\mathrm{d}_{\mathrm{A}}$ is an extended distance on $\mathrm{A}(X, \mathrm{~d})$ is a consequence of the previous results (applied to the topology induced by $d$ ). Let us now consider the two properties (X1) and (X2) of Definition 2.3 separately.

Proof of property $(X 1)$. For every $J \in \mathbb{N}$, let us denote by $\mathcal{P}_{J} \subset[0,1]^{J+1}$ the collection of all partitions $P=\left(t_{0}, t_{1}, \cdots t_{J}\right)$ with $0=t_{0} \leq t_{1} \leq t_{2} \leq \cdots \leq t_{J}=1$. For every $P \in \mathcal{P}_{J}$ and every finite collection of functions $\mathcal{F}=\left(f_{j}\right)_{j=1}^{J} \subset \operatorname{Lip}_{b}(X, \tau, \mathrm{d})$ we consider the functions $F_{P, \mathcal{F}}, F_{\mathcal{F}}$ defined by

$$
F_{\left(t_{0}, t_{1}, \cdots, t_{J}\right), \mathcal{F}}(\gamma):=\max _{1 \leq j \leq J} \max _{t \in\left[t_{j-1}, t_{j}\right]} f_{j}(\gamma(t)), \quad F_{\mathcal{F}}(\gamma)=\min _{P \in \mathcal{P}_{J}} F_{P, \mathcal{F}}(\gamma)
$$

By fixing the uniform partition $P_{J}=(0,1 / J, 2 / J, \cdots, j / J, \cdots, 1)$ as a reference, it is not difficult to check that

$$
F_{\mathcal{F}}(\gamma)=\min _{\gamma^{\prime} \sim \gamma} F_{P_{J}, \mathcal{F}}\left(\gamma^{\prime}\right)
$$

Since $F_{\mathcal{F}}$ only depends on the equivalence class of $\gamma$, it induces a map (denoted by $\bar{F}_{\mathcal{F}}$ ) on $\mathrm{A}(X, \mathrm{~d})$.

Claim: $\bar{F}_{\mathcal{F}}$ is $\tau_{\mathrm{A}}$-continuous. It is sufficient to show that $F_{\mathcal{F}}$ is $\tau_{\mathrm{C}}$ continuous. Let us consider the corresponding map $H: \mathcal{P}_{J} \times \mathrm{C}\left([0,1] ; \mathbb{R}^{J}\right) \rightarrow \mathbb{R}$,

$$
H\left(\left(t_{0}, t_{1}, \cdots, t_{N}\right), \boldsymbol{f}\right):=\max _{1 \leq j \leq J} \max _{t \in\left[t_{j-1}, t_{j}\right]} f_{j}(t), \quad \boldsymbol{f}:=\left(f_{1}, \cdots, f_{J}\right) \in \mathrm{C}\left([0,1] ; \mathbb{R}^{J}\right) .
$$

$H$ is continuous map (where $P_{J}$ is endowed with the product topology of $[0,1]^{J+1}$ ); since $P_{J}$ is compact, also $\tilde{H}(\boldsymbol{f}):=\min _{\left(t_{0}, t_{1}, \cdots, t_{N}\right) \in P_{J}} H\left(\left(t_{0}, t_{1}, \cdots, t_{N}\right), \boldsymbol{f}\right)$ is continuous. Since $F_{\mathcal{F}}(\gamma)=$ $\tilde{H}(\boldsymbol{f} \circ \gamma)$ we conclude that $F_{\mathcal{F}}$ is $\tau_{\mathrm{C}}$-continuous.

Claim: $\bar{F}_{\mathcal{F}}$ is $\mathrm{d}_{\mathrm{A}}$-Lipschitz. Let $\left[\gamma_{i}\right] \in \mathrm{A}(X, \mathrm{~d}), \varepsilon>0$, and $\varrho \in \Sigma^{\prime}$ such that $\mathrm{d}_{\mathrm{A}}\left(\left[\gamma_{1}\right],\left[\gamma_{2}\right]\right) \geq$ $\mathrm{d}_{\mathrm{C}}\left(\gamma_{1} \circ \varrho, \gamma_{2}\right)-\varepsilon$. We select a sequence $P \in \mathcal{P}_{J}$ such that $\bar{F}\left(\left[\gamma_{2}\right]\right)=F_{P, \mathcal{F}}\left(\gamma_{2}\right)$ and we observe that

$$
\begin{aligned}
\bar{F}_{\mathcal{F}}\left(\left[\gamma_{1}\right]\right)-\bar{F}_{\mathcal{F}}\left(\left[\gamma_{2}\right]\right) & \leq F_{\varrho(P), \mathcal{F}}\left(\gamma_{1}\right)-F_{P, \mathcal{F}}\left(\gamma_{2}\right) \leq F_{P, \mathcal{F}}\left(\gamma_{1} \circ \varrho\right)-F_{P, \mathcal{F}}\left(\gamma_{2}\right) \\
& \leq L \mathrm{~d}_{\mathrm{C}}\left(\gamma_{1} \circ \varrho, \gamma_{2}\right) \leq L\left(\mathrm{~d}_{\mathrm{A}}\left(\left[\gamma_{1}\right],\left[\gamma_{2}\right]\right)+\varepsilon\right),
\end{aligned}
$$


where $L$ is the greatest Lipschitz constant of the functions $f_{j}$. Since $\varepsilon>0$ is arbitrary and we can invert the order of $\gamma_{1}$ and $\gamma_{2}$ we conclude that $\bar{F}_{\mathcal{F}}$ is $\mathrm{d}_{\mathrm{A}}$-Lipschitz.

Conclusion. Now, if $U$ is saturated open set containing $\gamma_{0}$, thanks to Remark 3.1 we can find a collection of open sets $U_{j} \subset X$ such that

$$
\mathrm{U} \supset \mathrm{U}^{\prime}=\left\{\gamma \in \mathrm{C}([a, b] ; X): \text { there exists }\left(t_{0}^{\prime}, t_{1}^{\prime}, \cdots, t_{J}^{\prime}\right) \in \mathcal{P}_{J}: \gamma\left(\left[t_{j-1}^{\prime}, t_{j}^{\prime}\right]\right) \subset U_{j}\right\}, \quad \gamma_{0} \in \mathrm{U}^{\prime} \text {; }
$$

in particular, there exist $\left(t_{0}, t_{1}, \cdots, t_{J}\right) \in \mathcal{P}_{J}$ such that $\gamma_{0}\left(\left[t_{j-1}, t_{j}\right]\right) \subset U_{j}$.

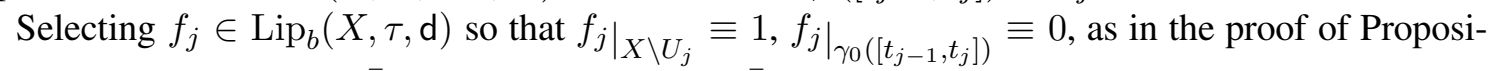
tion 3.2, we conclude that $\bar{F}_{\mathcal{F}}\left(\left[\gamma_{0}\right]\right)=F_{\mathcal{F}}\left(\gamma_{0}\right)=0,\left.\bar{F}_{\mathcal{F}}\right|_{\mathrm{A}(X, \mathrm{~d}) \backslash \mathrm{U}} \geq 1$.

Proof of property (X2). By Lemma 2.4 we can find a directed set $I$ and a monotone family $\left(\mathrm{d}_{i}\right)_{i \in I}$ of $\tau$-continuous, bounded semidistances such that $\mathrm{d}=\sup _{I} \mathrm{~d}_{i}$. We can therefore introduce the corresponding continuous and bounded semidistances $\mathrm{d}_{\mathrm{C}, i}$ on $\mathrm{C}([0,1] ; X)$ and $\mathrm{d}_{\mathrm{A}, i}$ on $\mathrm{A}(X)$ by

$$
\mathrm{d}_{\mathrm{C}, i}\left(\gamma_{1}, \gamma_{2}\right):=\sup _{t \in[0,1]} \mathrm{d}_{i}\left(\gamma_{1}(t), \gamma_{2}(t)\right), \quad \mathrm{d}_{\mathrm{A}, i}\left(\left[\gamma_{1}\right],\left[\gamma_{2}\right]\right):=\inf \left\{\mathrm{d}_{\mathrm{C}, i}\left(\gamma_{1}^{\prime}, \gamma_{2}^{\prime}\right): \gamma_{j}^{\prime} \sim \gamma_{j}\right\}
$$

Let us first notice that for every $\gamma_{1}, \gamma_{2} \in \mathrm{C}([0,1] ; X)$ we have

$$
\mathrm{d}_{\mathrm{C}}\left(\gamma_{1}, \gamma_{2}\right)=\lim _{i \in I} \mathrm{~d}_{\mathrm{C}, i}\left(\gamma_{1}, \gamma_{2}\right)=\sup _{i \in I} \mathrm{~d}_{\mathrm{C}, i}\left(\gamma_{1}, \gamma_{2}\right) \text {. }
$$

By claim (b) of Corollary 3.5 the semidistances $\mathrm{d}_{\mathrm{A}, i}$ are $\tau_{\mathrm{A}}$-continuous. Let us now fix $\gamma_{1}, \gamma_{2} \in$ $\mathrm{C}([0,1] ;(X, \mathrm{~d}))$ with $\mathrm{d}$-modulus of continuity $\omega_{\gamma_{1}}, \omega_{\gamma_{2}}$ as in $(3.21)$; by the reparametrization Theorem 3.4 we can find $\varrho_{1, i}, \varrho_{2, i} \in \Sigma_{2}$ such that

$$
\mathrm{d}_{\mathrm{A}, i}\left(\left[\gamma_{1}\right],\left[\gamma_{2}\right]\right)=\mathrm{d}_{\mathrm{C}, i}\left(\gamma_{1} \circ \varrho_{1, i}, \gamma_{2} \circ \varrho_{2, i}\right) \quad \text { for every } i \in I .
$$

Since $\Sigma_{2}$ is compact, we can find a directed subset $J$ and a monotone final map $h: J \rightarrow I$ such that the subnets $j \mapsto \varrho_{1, h(j)}$ and $j \mapsto \varrho_{2, h(j)}$ are convergent to elements $\varrho_{1}, \varrho_{2} \in \Sigma_{2}$. By (3.29), for every $\varepsilon>0$ we may find $i_{0} \in I$ such that

$$
\mathrm{d}_{\mathrm{A}}\left(\left[\gamma_{1}\right],\left[\gamma_{2}\right]\right) \leq \mathrm{d}_{\mathrm{C}}\left(\gamma_{1} \circ \varrho_{1}, \gamma_{2} \circ \varrho_{2}\right) \leq \mathrm{d}_{\mathrm{C}, i}\left(\gamma_{1} \circ \varrho_{1}, \gamma_{2} \circ \varrho_{2}\right)+\varepsilon \quad \text { for every } i \succeq i_{0} .
$$

On the other hand, there exists $j_{0} \in J$ such that $h\left(j_{0}\right) \succeq i_{0}$ and for every $j \succeq j_{0}$

$$
\sup _{t \in[0,1]}\left|\varrho_{1, h(j)}(t)-\varrho_{1}(t)\right| \leq \varepsilon, \quad \sup _{t \in[0,1]}\left|\varrho_{2, h(j)}(t)-\varrho_{2}(t)\right| \leq \varepsilon,
$$

so that for every $j \succeq j_{0}$

$$
\mathrm{d}_{\mathrm{C}, h(j)}\left(\gamma_{1} \circ \varrho_{1, h(j)}, \gamma_{2} \circ \varrho_{2, h(j)}\right) \geq \mathrm{d}_{\mathrm{C}, h(j)}\left(\gamma_{1} \circ \varrho_{1}, \gamma_{2} \circ \varrho_{2}\right)-\omega_{\gamma_{1}}(\varepsilon)-\omega_{\gamma_{2}}(\varepsilon) .
$$

Combining (3.30) with (3.31) we thus obtain

$$
\mathrm{d}_{\mathrm{A}}\left(\left[\gamma_{1}\right],\left[\gamma_{2}\right]\right) \leq \mathrm{d}_{\mathrm{A}, h(j)}\left(\left[\gamma_{1}\right],\left[\gamma_{2}\right]\right)+\varepsilon+\omega_{\gamma_{1}}(\varepsilon)+\omega_{\gamma_{2}}(\varepsilon)
$$

for every $j \succeq j_{0}$. Passing to the limit w.r.t. $j \in J$ we get

$$
\begin{aligned}
\mathrm{d}_{\mathrm{A}}\left(\left[\gamma_{1}\right],\left[\gamma_{2}\right]\right) & \leq \lim _{j \in J} \mathrm{~d}_{\mathrm{A}, h(j)}\left(\left[\gamma_{1}\right],\left[\gamma_{2}\right]\right)+\varepsilon+\omega_{\gamma_{1}}(\varepsilon)+\omega_{\gamma_{2}}(\varepsilon) \\
& =\lim _{i \in I} \mathrm{~d}_{\mathrm{A}, i}\left(\left[\gamma_{1}\right],\left[\gamma_{2}\right]\right)+\varepsilon+\omega_{\gamma_{1}}(\varepsilon)+\omega_{\gamma_{2}}(\varepsilon),
\end{aligned}
$$

which yields $\mathrm{d}_{\mathrm{A}}\left(\left[\gamma_{1}\right],\left[\gamma_{2}\right]\right)=\lim _{i \in I} \mathrm{~d}_{\mathrm{A}, i}\left(\left[\gamma_{1}\right],\left[\gamma_{2}\right]\right)$ since $\varepsilon>0$ is arbitrary. 
Corollary 3.7. If $\tau^{\prime}$ is an auxiliary topology for $(X, \tau, \mathrm{d})$ according to Definition 2.6 then $\tau_{\mathrm{A}}^{\prime}$ is an auxiliary topology for $\left(\mathrm{A}(X, \mathrm{~d}), \tau_{\mathrm{A}}, \mathrm{d}_{\mathrm{A}}\right)$.

Proof. Properties $(\mathrm{A} 1,2)$ of Definition 2.6 are immediate. We now select an increasing sequence $\mathrm{d}_{n}$ of $\tau^{\prime}$-continuous distances satisfying (2.28'); arguing as in the previous proof we obtain that $\mathrm{d}_{\mathrm{A}}\left(\left[\gamma_{1}\right],\left[\gamma_{2}\right]\right)=\sup _{n \in \mathbb{N}} \mathrm{d}_{\mathrm{A}, n}\left(\left[\gamma_{1}\right],\left[\gamma_{2}\right]\right)$ for every $\gamma_{1}, \gamma_{2} \in \mathrm{C}([0,1] ;(X, \mathrm{~d}))$, which precisely yields (A3') for $\tau_{\mathrm{A}}^{\prime}$.

\subsection{Rectifiable arcs}

If $\delta$ is an extended semidistance in $X, \gamma:[a, b] \rightarrow X$ and $J \subset[a, b]$ we set

$$
\operatorname{Var}_{\delta}(\gamma ; J):=\sup \left\{\sum_{j=1}^{N} \delta\left(\gamma\left(t_{j}\right), \gamma\left(t_{j-1}\right)\right):\left\{t_{j}\right\}_{j=0}^{N} \subset J, \quad t_{0}<t_{1}<\cdots<t_{N}\right\} .
$$

$\mathrm{BV}([a, b] ;(X, \delta))$ will denote the space of maps $\gamma:[a, b] \rightarrow X$ such that $\operatorname{Var}_{\delta}(\gamma ;[a, b])<\infty$ (we will omit $\delta$ in the case of the distance d). We will set

$$
\ell(\gamma):=\operatorname{Var}_{\mathrm{d}}(\gamma ;[a, b]), \quad V_{\gamma}(t):=\operatorname{Var}_{\mathrm{d}}(\gamma ;[a, t]) \quad t \in[a, b],
$$

and

$$
V_{\gamma, \ell}(t):=\ell(\gamma)^{-1} V_{\gamma}(t) \quad \text { whenever } \ell(\gamma)>0, \quad V_{\gamma, \ell}(t):=0 \quad \text { if } \ell(\gamma)=0 ;
$$

notice that if $\ell(\gamma)=0$ then $\gamma$ is constant.

Lemma 3.8. Let $(X, \tau, \mathrm{d})$ be an extended metric-topological space. If $\gamma \in \mathrm{C}([a, b] ;(X, \tau))$ satisfies $\operatorname{Var}_{\mathrm{d}}(\gamma ;[a, b])<\infty$ then $\gamma \in \mathrm{C}([a, b] ;(X, \mathrm{~d}))$, and its variation map $V_{\gamma}$ is continuous in $[a, b]$. We will set

$$
\operatorname{BVC}([a, b] ; X):=\operatorname{BV}([a, b] ;(X, \mathrm{~d})) \cap \mathrm{C}([a, b] ;(X, \tau))=\mathrm{BV}([a, b] ;(X, \mathrm{~d})) \cap \mathrm{C}([a, b] ;(X, \mathrm{~d})) .
$$

Proof. Thanks to the obvious estimate

$$
\mathrm{d}(\gamma(r), \gamma(s)) \leq V_{\gamma}(s)-V_{\gamma}(r) \text { for every } r, s \in[a, b], r \leq s,
$$

it is easy to check $\gamma$ is d-continuous at every continuity point of $V_{\gamma}$. Let us fix $r, s, t \in(a, b]$ with $r<s<t$; passing to the limit in the inequality (3.36) keeping $r$ fixed and letting $s \uparrow t$ we get by the $\tau$-continuity of $\gamma$, the lower semicontinuity of $\mathrm{d}$ and the monotonicity of $V_{\gamma}$

$$
\mathrm{d}(\gamma(t), \gamma(r)) \leq V_{\gamma}(t-)-V_{\gamma}(r) \quad V_{\gamma}(t-):=\lim _{s \uparrow t} V_{\gamma}(s) .
$$

We thus obtain $\lim _{r \uparrow t} \mathrm{~d}(\gamma(t), \gamma(r))=0$. A similar argument yields the right continuity of $\gamma$ and therefore the continuity of $V_{\gamma}$.

Since the length functional $\gamma \mapsto \ell(\gamma)$ is lower semicontinuous with respect to the compact-open topology of $\mathrm{C}([a, b] ;(X, \tau))$, the set $\mathrm{BVC}([a, b] ; X)$ is an $F_{\sigma}$ in $\mathrm{C}([a, b] ;(X, \tau))$. We consider two other subsets:

$$
\mathrm{BVC}_{c}([a, b] ; X):=\left\{\gamma \in \operatorname{BVC}([a, b] ; X): V_{\gamma}(t)=\ell(\gamma)(t-a)\right\}
$$


whose curves have constant velocity, and

$$
\mathrm{BVC}_{k}([a, b] ; X):=\{\gamma \in \operatorname{BVC}([a, b] ; X): \ell(\gamma)>k\} \quad k \in[0,+\infty[,
$$

whose curves are non-constant $\mathrm{BVC}_{c}([a, b] ; X)$ is a Borel subset of $\mathrm{BVC}([a, b] ; X)$ since it can be equivalently characterized by the condition

$$
\gamma \in \mathrm{BVC}_{c}([a, b] ; X) \Leftrightarrow \operatorname{Lip}(\gamma ;[a, b]) \leq \ell(\gamma),
$$

and the maps Lip and $\ell$ are lower semicontinuous. $\mathrm{BVC}_{0}([a, b] ; X)$ is open in $\mathrm{BVC}_{c}([a, b] ; X)$.

Corollary 3.9. If $(X, \tau, \mathrm{d})$ is Polish then $\operatorname{BVC}([a, b] ; X), \mathrm{BVC}_{c}([a, b] ; X), \mathrm{BVC}_{k}([a, b] ; X), k \geq 0$, are Lusin spaces. If $(X, \tau, \mathrm{d})$ admits an auxiliary topology $\tau^{\prime}$ (in particular if $(X, \tau)$ is Souslin) then they are $\mathscr{F}\left(\mathrm{C}([a, b] ; X), \tau_{\mathrm{C}}\right)$ analytic sets.

Proof. The first statement follows by the fact that Borel sets in a Polish space (in this case the space $\left(\mathrm{C}\left([a, b] ;(X, \tau), \tau_{\mathrm{C}}\right)\right)$ are Lusin.

The second claim is obvious for the $F_{\sigma}$ set $\mathrm{BVC}([a, b] ; X)$; in the case of $\mathrm{BVC}_{c}([a, b] ; X)$ and $\mathrm{BVC}_{k}([a, b] ; X)$, it follows by the fact that they are Borel sets in the metrizable and separable space $\left(\mathrm{C}\left([a, b] ;\left(X, \tau^{\prime}\right), \tau_{\mathrm{C}}^{\prime}\right)\right.$, thus are $\mathscr{F}$-analytic (see (A3) in $\S$ A.3) for the coarser topology $\tau_{\mathrm{C}}^{\prime}$, and thus $\mathscr{F}$-analytic also with respect to $\tau_{\mathrm{C}}$.

If $f \in \mathrm{C}(X)$ and $\gamma \in \mathrm{BVC}([a, b] ; X)$ then the integral $\int_{\gamma} f$ is well defined by Riemann-Stieltjes integration of $f \circ \gamma$ with respect to $\mathrm{d} V_{\gamma}$; it can also be obtained as the limit of the Riemann sums

$$
\begin{aligned}
\int_{\gamma} f & =\lim _{\tau(P) \downarrow 0} \sum_{j=1}^{N} f\left(\gamma\left(\xi_{j}\right)\right) \mathrm{d}\left(\gamma\left(t_{j}\right), \gamma\left(t_{j-1}\right)\right): \\
P & =\left\{t_{0}=a \leq \xi_{1} \leq t_{1} \leq \xi_{2} \leq \cdots \leq t_{N-1} \leq \xi_{N} \leq t_{N}=b\right\}, \quad \tau(P):=\sup _{j}\left|t_{j}-t_{j-1}\right|
\end{aligned}
$$

since in (3.40) is equivalent to use $\mathrm{d}\left(\gamma\left(t_{j}\right), \gamma\left(t_{j-1}\right)\right)$ or $V_{\gamma}\left(t_{j}\right)-V_{\gamma}\left(t_{j-1}\right)$.

Notice that for every $\gamma \in \operatorname{BVC}([a, b] ; X)$ the map $V_{\gamma}:[a, b] \rightarrow[0, \ell(\gamma)]$ is continuous and surjective and

there exists a unique $\ell(\gamma)$-Lipschitz map $R_{\gamma} \in \mathrm{BVC}_{c}([0,1] ; X)$ such that $\gamma=R_{\gamma} \circ\left(V_{\gamma, \ell}\right)$,

with $\left|R_{\gamma}^{\prime}\right|(s)=\ell(\gamma)$ a.e. and

$$
\int_{\gamma} f=\int_{0}^{1} f\left(R_{\gamma}(s)\right)\left|R_{\gamma}^{\prime}\right|(s) \mathrm{d} s=\ell(\gamma) \int_{0}^{1} f\left(R_{\gamma}(s)\right) \mathrm{d} s .
$$

Denoting by $\vartheta_{\gamma}:[0,1] \rightarrow[a, b]$ the right-continuous pseudo inverse of $V_{\gamma, \ell}$ (when $\ell(\gamma)>0$ )

$$
\vartheta_{\gamma}(s):=\max \left\{t \in[a, b]: V_{\gamma, \ell}(t)=s\right\} \quad s \in[0,1], \quad \text { so that } V_{\gamma, \ell}\left(\vartheta_{\gamma}(s)\right)=s \quad \text { in }[0,1]
$$

we have $R_{\gamma}=\gamma \circ \vartheta_{\gamma}$. When $\ell(\gamma)=0$ we set $\vartheta_{\gamma}(s) \equiv b$, and we still have $R_{\gamma}=\gamma \circ \vartheta_{\gamma}$. We also notice that

$$
\int_{\gamma} f=\int f \mathrm{~d} \nu_{\gamma} \quad \text { where } \quad \nu_{\gamma}:=\ell(\gamma)\left(R_{\gamma}\right)_{\sharp}\left(\mathscr{L}^{1}\llcorner[0,1]) ;\right.
$$

by (3.44) it is possible to extend the integral to every bounded or nonnegative Borel map $f: X \rightarrow \mathbb{R}$. 
Lemma 3.10. Let $\gamma \in \operatorname{BVC}([0,1] ; X)$ and let $\vartheta:[0,1] \rightarrow[0,1]$ be an increasing map.

(a) The map $\tilde{\gamma}:=\gamma \circ \vartheta$ belongs to $\operatorname{BV}([0,1] ;(X, \mathrm{~d}))$ and

$$
V_{\gamma \circ \vartheta, \ell}(t) \leq V_{\gamma, \ell}(\vartheta(t)) \quad \text { for every } t \in[0,1] \text {. }
$$

(b) If $\tilde{\gamma} \in \mathrm{C}([0,1] ;(X, \tau)), \ell(\tilde{\gamma})=\ell(\gamma)$, and $V_{\gamma, \ell}$ is strictly increasing, then $\vartheta \in \Sigma$.

(c) If $\vartheta \in \Sigma$ then $\tilde{\gamma}$ still belongs to $\operatorname{BVC}([0,1] ; X)$ and

$$
V_{\tilde{\gamma}, \ell}=V_{\gamma \circ \vartheta, \ell}=V_{\gamma, \ell} \circ \vartheta
$$

and

$$
\ell(\tilde{\gamma})=\ell(\gamma), \quad R_{\tilde{\gamma}}=R_{\gamma}, \quad \int_{\gamma} f=\int_{\tilde{\gamma}} f
$$

Proof. Claims (a) and (c) follow easily by the definition (3.33), the characterization of $R_{\gamma}$ and (3.42).

In order to check Claim (b), we choose a point $t \in(0,1)$ (the argument for the case $t=0$ or $t=1$ can be easily adapted) and we set $r_{-}=\lim _{s \uparrow t} \vartheta(s), r_{+}=\lim _{s \downarrow t} \vartheta(s)$. The identity $\tilde{\gamma}=\gamma \circ \vartheta$ and the continuity of $\tilde{\gamma}$ yield $\gamma\left(r_{-}\right)=\gamma\left(r_{+}\right)$and

$$
\begin{aligned}
\ell(\tilde{\gamma}) & =\operatorname{Var}_{\mathrm{d}}(\tilde{\gamma} ;[0, t])+\operatorname{Var}_{\mathrm{d}}(\tilde{\gamma} ;[t, 1]) \leq \operatorname{Var}_{\mathrm{d}}\left(\gamma ;\left[0, r_{-}\right]\right)+\operatorname{Var}_{\mathrm{d}}\left(\gamma ;\left[r_{+}, 1\right]\right) \\
& =\ell(\gamma)-\operatorname{Var}_{\mathrm{d}}\left(\gamma ;\left[r_{-}, r_{+}\right]\right)=\ell(\gamma)\left(1+V_{\gamma, \ell}\left(r_{-}\right)-V_{\gamma, \ell}\left(r_{+}\right)\right) .
\end{aligned}
$$

We deduce that $V_{\gamma, \ell}\left(r_{-}\right)=V_{\gamma, \ell}\left(r_{+}\right)$so that $r_{-}=r_{+}$. As similar argument shows that $\vartheta(i)=i$, $i=0,1$, so that $\vartheta$ is surjective.

On $\operatorname{BVC}([0,1] ; X)$ we introduce the equivalence relation (3.11) and we will denote by $\operatorname{RA}(X, \mathrm{~d})$ (or simply $\mathrm{RA}(X))$ the quotient space $\mathrm{BVC}([0,1] ; X) / \sim$ endowed with the quotient topology $\tau_{\mathrm{A}}$ induced by $\mathrm{C}([0,1] ;(X, \tau))$ and with the extended distance

$$
\mathrm{d}_{\mathrm{A}}\left(\left[\gamma_{1}\right],\left[\gamma_{2}\right]\right):=\inf \left\{\mathrm{d}_{\mathrm{C}}\left(\gamma_{1}^{\prime}, \gamma_{2}^{\prime}\right): \gamma_{i}^{\prime} \sim \gamma_{i}\right\}=\inf _{\varrho_{i} \in \Sigma} \mathrm{d}_{\mathrm{C}}\left(\gamma_{1} \circ \varrho_{1}, \gamma_{2} \circ \varrho_{2}\right)
$$

as in (3.12). By Proposition 3.6 the space $\left(\mathrm{RA}(X, \mathrm{~d}), \tau_{\mathrm{A}}, \mathrm{d}_{\mathrm{A}}\right)$ is an extended metric-topological space.

Lemma 3.11 (Reparametrizations of rectifiable arcs). Let $(X, \tau, \mathrm{d})$ be an extended metric-topological space. We have:

(a) If $\gamma \in \mathrm{BVC}([0,1] ; X), \gamma^{\prime} \in \mathrm{C}([0,1] ; X)$ and $\gamma^{\prime} \sim \gamma$ then $\gamma^{\prime} \in \mathrm{BVC}([0,1] ; X)$.

(b) For every $\gamma, \gamma^{\prime} \in \operatorname{BVC}([0,1] ; X)$ we have

$$
\gamma \sim \gamma^{\prime} \quad \Leftrightarrow \quad R_{\gamma}=R_{\gamma^{\prime}}
$$

and all the curves $\gamma^{\prime}$ equivalent to $\gamma$ can be described as $\gamma^{\prime}=R_{\gamma} \circ \sigma$ for some $\sigma \in \Sigma$.

(c) For every $\gamma_{i} \in \operatorname{BVC}([0,1] ; X)$ the distance $\mathrm{d}_{\mathrm{A}}$ satisfies $(3.16 \mathrm{a}, \mathrm{b}, \mathrm{c})$ and we have

$$
\begin{aligned}
\mathrm{d}_{\mathrm{A}}\left(\gamma_{1}, \gamma_{2}\right) & =\inf _{\sigma \in \Sigma^{\prime}} \mathrm{d}_{\mathrm{C}}\left(R_{\gamma_{1}}, R_{\gamma_{2}} \circ \sigma\right) \\
& =\min _{\varrho_{i} \in \Sigma_{2}} \mathrm{~d}_{\mathrm{C}}\left(R_{\gamma_{1}} \circ \varrho_{1}, R_{\gamma_{2}} \circ \varrho_{2}\right)
\end{aligned}
$$


(d) The function $\ell$ and the evaluation maps $\mathrm{e}_{0}, \mathrm{e}_{1}$ are invariant w.r.t. parametrizations, so that we will still denote by $\ell$ and $\mathrm{e}_{0}, \mathrm{e}_{1}$ the corresponding quotient maps. $\ell: \mathrm{A}(X, \tau) \rightarrow[0,+\infty]$ is $\tau_{\mathrm{A}}$-lower semicontinuous and $\mathrm{e}_{0}, \mathrm{e}_{1}: \mathrm{A}(X, \tau) \rightarrow X$ are continuous.

(e) If $f: X \rightarrow[0,+\infty]$ is lower semicontinuous then the map $\gamma \mapsto \int_{\gamma} f$ only depends on $[\gamma]$ and it is lower semicontinuous w.r.t. $\tau_{\mathrm{A}}$ in $\mathrm{RA}(X, \mathrm{~d})$.

Proof. (a) Since $\gamma^{\prime} \circ \sigma^{\prime}=\gamma \circ \sigma$ for some $\sigma, \sigma^{\prime} \in \Sigma$, we have $\ell\left(\gamma^{\prime}\right)=\ell(\gamma)<\infty$ by (3.47).

(b) The right implication $\Rightarrow$ in (3.49) follows by (3.47). In order to prove the converse implication it is not restrictive to suppose $\ell(\gamma)=\ell\left(\gamma^{\prime}\right)>0$; we observe that there exist $\sigma, \sigma^{\prime} \in \Sigma$ so that $R_{\gamma} \circ \sigma=R_{\gamma^{\prime}} \circ \sigma^{\prime}=\gamma^{\prime \prime}$ and therefore $R_{\gamma^{\prime \prime}}=R_{\gamma} \circ\left(\sigma \circ \vartheta_{\gamma^{\prime \prime}}\right)$. Thanks to the second claim of Lemma 3.10 we deduce that $\varrho:=\sigma \circ \vartheta_{\gamma^{\prime \prime}} \in \Sigma$ is continuous and surjective. Recalling that for every $\gamma \in \operatorname{BVC}([0,1] ; X) V_{R_{\gamma}, \ell}(t)=t$ by construction, (3.46) yields

$$
t=V_{R_{\gamma^{\prime \prime}}, \ell}(t)=V_{R_{\gamma} \varrho \varrho, \ell}(t)=V_{R_{\gamma}, \ell}(\varrho(t))=\varrho(t) \quad \text { for every } t \in[0,1] .
$$

(c) is an immediate consequence of Theorem 3.4 for $\delta:=\mathrm{d}$.

(d) The function $\ell$ is lower semicontinuous w.r.t. the $\tau_{\mathrm{C}}$-topology being the supremum of lower semicontinuous functions by (3.33). Since $\ell(\gamma)$ is independent on the choice of a representative in $[\gamma]$, it is also lower semicontinuous w.r.t. the $\tau_{\mathrm{A}}$ topology. A similar argument holds for the initial and final evaluation maps $e_{0}, e_{1}$.

(e) If $f$ is continuous, we use the representation of the integral by Riemann sums

$$
\int_{\gamma} f=\sup \sum_{j=1}^{N}\left(\inf _{t \in\left[t_{j-1}, t_{j}\right]} f(\gamma(t))\right) \mathrm{d}\left(\gamma\left(t_{j}\right), \gamma\left(t_{j-1}\right)\right): t_{0}=0<t_{1}<\cdots<t_{N-1}<t_{N}=1
$$

which exhibits $\int_{\gamma} f$ as the supremum of $\tau_{\mathrm{C}}$ lower semicontinuous functions. The invariance of the integral w.r.t. reparametrization yields the $\tau_{\mathrm{A}}$ lower semicontinuity.

When $f$ is $\tau$-lower semicontinuous, we can represent it as the supremum of the (directed) set

$$
f(x)=\sup _{g \in F} g(x), \quad F:=\left\{g \in \mathrm{C}_{b}(X), 0 \leq g \leq f\right\} .
$$

Since $\nu_{\gamma}$ is a Radon measure, we have

$$
\int_{\gamma} f=\int_{X} f \mathrm{~d} \nu_{\gamma}=\sup _{g \in F} \int_{X} g \mathrm{~d} \nu_{\gamma}=\sup _{g \in F} \int_{\gamma} g
$$

Lemma 3.12. $\mathrm{RA}(X, \mathrm{~d})$ is an $F_{\sigma}$-subset of $\left(\mathrm{A}(X, \tau), \tau_{\mathrm{A}}\right)$.

If $(X, \tau)$ is a Polish space then $\left(\mathrm{RA}(X, \mathrm{~d}), \tau_{\mathrm{A}}\right)$ is a Lusin space. If $(X, \tau, \mathrm{d})$ admits an auxiliary topology $\tau^{\prime}$ (in particular if $(X, \tau)$ is Souslin) then for every $k \geq 0$ the (relatively) open subsets

$$
\operatorname{RA}_{k}(X, \mathrm{~d}):=\{\gamma \in \operatorname{RA}(X, \mathrm{~d}): \ell(\gamma)>k\}
$$

are $\mathscr{F}(\mathrm{RA}(X, \mathrm{~d}))$-analytic set for the $\tau_{\mathrm{A}}^{\prime}$ and the $\tau_{\mathrm{A}}$-topology.

Proof. Notice that $\operatorname{RA}(X, \mathrm{~d})$ can be equivalently identified with the $F_{\sigma}$-subset of $\mathrm{A}(X, \tau)$ and of $\mathrm{A}(X, \mathrm{~d})$ defined by $\{\gamma \in \mathrm{A}(X, \tau): \ell(\gamma)<\infty\}$ with the induced topology $\tau_{\mathrm{A}}$ and the extended distance $\mathrm{d}_{\mathrm{A}}$ of $\mathrm{A}(X, \mathrm{~d})$. From this point of view, $\mathrm{RA}(X, \mathrm{~d})$ is a $F_{\sigma}$ subset (i.e. it is the countable union 
of closed sets and therefore it is also a Borel set) since $\operatorname{RA}(X, \mathrm{~d})=\cup_{k \in \mathbb{N}}\{\gamma \in \mathrm{A}(X, \tau): \ell(\gamma) \leq k\}$ and the map $\ell: \mathrm{A}(X, \tau) \rightarrow[0,+\infty]$ is lower semicontinuous with respect to $\tau_{\mathrm{A}}$ thanks to (d) of the previous Lemma 3.11. Since $\ell$ is also $\tau_{\mathrm{A}}^{\prime}$-1.s.c. and $\tau_{\mathrm{A}}^{\prime}$ is metrizable, all the sets $\operatorname{RA}_{k}(X, \mathrm{~d})$ are $F_{\sigma}$ and thus $\mathscr{F}$-analytic.

Finally, if $(X, \tau)$ is Polish, Corollary 3.9 shows that $\left(\mathrm{BVC}_{c}([0,1] ; X), \tau_{\mathrm{C}}\right)$ is a Lusin space. Lemma 3.11 shows that the quotient map $\mathrm{q}$ is a continuous bijection of $\left(\mathrm{BVC}_{c}([0,1] ; X), \tau_{\mathrm{C}}\right)$ onto $\left(\mathrm{RA}(X, \mathrm{~d}), \tau_{\mathrm{A}}\right)$, so that the latter is Lusin as well.

We conclude this section with a list of useful properties concerning the compactness in $\mathrm{RA}(X, \mathrm{~d})$ and the continuity of the map $\gamma \mapsto \nu_{\gamma}$ defined by (3.44). For every $t \in[0,1]$ we also introduce the arc-length evaluation maps

$$
\hat{\mathrm{e}}_{t}: \operatorname{RA}(X, \mathrm{~d}) \rightarrow X, \quad \hat{\mathrm{e}}_{t}:=\hat{\mathrm{e}}_{t} \circ R, \quad \hat{\mathrm{e}}_{t}(\gamma)=R_{\gamma}(t) \quad \text { for every } \gamma \in \operatorname{RA}(X, \mathrm{~d}) .
$$

When $t=0,1$ we still keep the notation $\gamma_{t}$ for the initial and final points $\mathrm{e}_{t}(\gamma)=\hat{\mathrm{e}}_{t}(\gamma)$.

Theorem 3.13. (a) If $\gamma_{i}, i \in I$, is a converging net in $\operatorname{RA}(X, \mathrm{~d})$ with $\gamma=\lim _{i \in I} \gamma_{i}$ and $\lim _{i \in I} \ell\left(\gamma_{i}\right)=$ $\ell(\gamma)$ then

$$
\lim _{i \in I} R_{\gamma_{i}}=R_{\gamma} \quad \text { in } \mathrm{C}([0,1] ; X), \quad \lim _{i \in I} \hat{\mathrm{e}}_{t}\left(\gamma_{i}\right)=\hat{\mathrm{e}}_{t}(\gamma) \quad \text { for every } t \in[0,1],
$$

and for every bounded and continuous function $f \in \mathrm{C}_{b}(X, \tau)$ we have

$$
\lim _{i \in I} \int_{\gamma_{i}} f=\int_{\gamma} f
$$

In particular, we have

$$
\lim _{i \in I} \nu_{\gamma_{i}}=\nu_{\gamma} \quad \text { weakly in } \mathcal{M}_{+}(X)
$$

(b) The map $\gamma \mapsto \nu_{\gamma}$ from $\mathrm{RA}(X, \mathrm{~d})$ to $\mathcal{M}_{+}(X)$ is universally Lusin measurable.

(c) If $i \mapsto \gamma_{i}$ converges to $\gamma$ in $\operatorname{RA}(X), \sup \ell\left(\gamma_{i}\right)<\infty$ and $\nu_{\gamma_{i}} \rightarrow \mu$ in $\mathcal{M}_{+}(X)$ with $\mu(X)>0$, then $\operatorname{supp}(\mu)=\gamma([0,1])$.

(d) The map $[\gamma] \mapsto R_{\gamma}$ is universally Lusin measurable from $\mathrm{RA}(X, \mathrm{~d})$ to $\mathrm{BVC}_{c}([0,1] ;(X, \mathrm{~d}))$ endowed with the topology $\tau_{\mathrm{C}}$ and it is also Borel if $\mathbb{X}$ has an auxiliary topology (in particular if $(X, \tau)$ is Souslin). For every $t \in[0,1]$ the maps $\hat{\mathrm{e}}_{t}: \mathrm{RA}(X, \mathrm{~d}) \rightarrow X$ are universally Lusin measurable (and Borel if $\mathbb{X}$ has an auxiliary topology).

(e) If $f \in \mathrm{B}_{b}(X)$ (or $f: X \rightarrow[0,+\infty]$ Borel) the map $\gamma \mapsto \int_{\gamma} f$ is Borel. In particular the family of measures $\left\{\nu_{\gamma}\right\}_{\gamma \in \mathrm{RA}(X)}$ is Borel.

(f) If $(X, \tau)$ is compact and $\Gamma \subset \mathrm{RA}(X, \mathrm{~d})$ satisfies $\sup _{\gamma \in \Gamma} \ell(\gamma)<+\infty$ then $\Gamma$ is relatively compact in $\mathrm{RA}(X, \mathrm{~d})$ w.r.t. the $\tau_{\mathrm{A}}$ topology.

(g) If $(X, \mathrm{~d})$ is complete and $\Gamma \subset \mathrm{RA}(X, \mathrm{~d})$ satisfies the following conditions:

1. $\sup _{\gamma \in \Gamma} \ell(\gamma)<+\infty$;

2. there exists a $\tau$-compact set $K \subset X$ such that $\mathrm{e}(\gamma) \cap K \neq \emptyset$ for every $\gamma \in \Gamma$;

3. $\left\{\nu_{\gamma}: \gamma \in \Gamma\right\}$ is equally tight, i.e. for every $\varepsilon>0$ there exists a $\tau$-compact set $K_{\varepsilon} \subset X$ such that $\nu_{\gamma}\left(X \backslash K_{\varepsilon}\right) \leq \varepsilon$ for every $\gamma \in \Gamma$, 
then $\Gamma$ is relatively compact in $\mathrm{RA}(X, \mathrm{~d})$ w.r.t. the $\tau_{\mathrm{A}}$ topology.

Proof. (a) In order to prove (3.54) we consider the compactification $(\hat{X}, \hat{\tau}, \hat{\mathrm{d}})$ given by Theorem 2.34 (here we can choose, e.g., $\mathscr{A}=\operatorname{Lip}_{b}(X, \tau, \mathrm{d})$; the measure $\mathfrak{m}$ does not play any role). Clearly the imbedding $\iota: X \rightarrow \hat{X}$ extends to a corresponding embedding of $\operatorname{RA}(X, \mathrm{~d})$ in $\operatorname{RA}(\hat{X}, \hat{\mathrm{d}})$, simply by setting $\hat{\gamma}(t):=\iota \circ \gamma(t)$ and considering the corresponding equivalence class. We can apply Proposition 3.3 to the net $i \mapsto R_{\hat{\gamma}_{i}}=\hat{R}_{\gamma_{i}}$ and we find a limit curve $\hat{R}_{*} \in \operatorname{Lip}([0,1] ;(\hat{X}, \hat{\mathrm{d}}))$ with respect to the topology $\hat{\tau}_{\mathrm{C}}$. Since the projection from $\mathrm{C}([0,1] ; \hat{X})$ to $\mathrm{A}(\hat{X})$ is continuous, we deduce that $\left[R_{*}\right]=\hat{\gamma}$ so that $\hat{R}_{*}$ takes values in $\iota(X)$ and therefore can be written as $\iota \circ R_{*}$ for a curve $R_{*} \in \operatorname{Lip}([0,1] ;(X, \mathrm{~d}))$ which is the limit of $R_{\gamma_{i}}$ in $\mathrm{C}([0,1] ; X)$. Passing to the limit in the identities

$$
\mathrm{d}\left(R_{\gamma_{i}}(r), R_{\gamma_{i}}(s)\right) \leq \ell\left(\gamma_{i}\right)|r-s| \quad \text { we get } \quad \mathrm{d}\left(R_{*}(r), R_{*}(s)\right) \leq \ell(\gamma)|r-s| .
$$

so that $R_{*}=R_{\gamma}$.

Let us prove (3.55). We set $m:=\inf f$ and $M=\sup f$ and we observe that $\ell(\gamma)=\int_{\gamma} \mathbb{1}$ so that the thesis follows by applying the lower semicontinuity property of Lemma 3.11 (e), to the functions $f-m$ and $M-f$.

(b) Let $\mu \in \mathcal{P}(\operatorname{RA}(X))$; since the function $\ell$ is lower semicontinuous in $X$, it is Lusin $\mu$-measurable and there exists a sequence of compact sets $K_{n} \subset X$ with $\lim _{n \rightarrow \infty} \mu\left(X \backslash K_{n}\right)=0$ such that the restriction of $\ell$ to $K_{n}$ is continuous. By the previous claim, the restriction of $\nu$ to $K_{n}$ is also continuous.

(c) Let $K:=\gamma([0,1])$; if $y \notin K$ then we can find a function $f \in \operatorname{Lip}_{b}(X, \tau, \mathrm{d})$ with values in $[0,1]$ such that $f_{\left.\right|_{K}} \equiv 0$ and $f(y)=1$. If $U:=\{x \in X: f(x)>1 / 2\}$ then there exists $i_{0} \in I$ such that $\gamma_{i}([0,1]) \cap \bar{U}=\emptyset$ for $i \succeq i_{0}$. It follows that $\nu_{\gamma_{i}}(U)=0$ and therefore

$$
\mu(U) \leq \liminf _{i \in I} \nu_{\gamma_{i}}(U)=0 .
$$

This shows that $\operatorname{supp}(\mu) \subset K$. If $K$ consists of an isolated point, the thesis then follows. On the other hand, if $K$ contains at least two points and $y \in K$ then for every open neighborhood $U$ of $y$ $\nu_{\gamma}(U)>0$ and therefore $\mu(U)>0$.

(d) The proof of universal measurability follows as in Claim b), by using the continuity property (3.54).

Let us now suppose that $\mathbb{X}$ admits an auxiliary topology $\tau^{\prime}$ (thus metrizable and separable) and let us prove that $R$ is Borel from $\operatorname{RA}(X)$ endowed with $\tau_{\mathrm{A}}^{\prime}$ to $\mathrm{C}([0,1] ; X)$ endowed with $\tau_{\mathrm{C}}$ (this implies the same property for the stronger topology $\tau_{\mathrm{A}}$ on $\left.\mathrm{RA}(X)\right)$. We observe that the map $J$ : $\gamma \rightarrow(\gamma, \ell(\gamma))$ is Borel from $\left(\mathrm{RA}(X), \tau_{\mathrm{A}}^{\prime}\right)$ to $\left(\mathrm{RA}(X) \times \mathbb{R}, \tau_{\mathrm{A}}^{\prime} \times \tau_{\mathbb{R}}\right)$ since the latter topology has a countable base of open sets (thus the Borel $\sigma$-algebra coincides with the product of the Borel $\sigma$-algebra of the factors) and each component of $J$ is Borel. On the other hand, $G:=\{(\gamma, r) \in \operatorname{RA}(X) \times \mathbb{R}$ : $r=\ell(\gamma)\}$ is Borel in $\mathrm{RA}(X) \times \mathbb{R}$ (with the product topology $\tau_{\mathrm{A}} \times \tau_{\mathbb{R}}$ ) [57, Chapter II, Lemma 12] and Claim (a) shows that the map $\tilde{R}: G \rightarrow \operatorname{RA}(X), \tilde{R}(\gamma, r):=R_{\gamma}$ is continuous in $G$, so that $R=\tilde{R} \circ J$ is a Borel map. Finally, since $\hat{\mathrm{e}}_{t}=\mathrm{e}_{t} \circ R$, the maps $\hat{\mathrm{e}}_{t}$ are Borel as well.

(e) Let us consider the set $H \subset \mathrm{B}_{b}(X)$ of functions $f$ such that $\gamma \mapsto \int_{\gamma} f$ is Borel. $H$ is clearly a vector space and contains the set $C:=\left\{\chi_{U}, U\right.$ open in $\left.X\right\}$, since the map $\gamma \mapsto \int_{\gamma} \chi_{U}$ is lower semicontinuous. Since $C$ is closed under multiplication, we can apply the criterium [25, Chap. I, Theorem 21], which shows that $H=\mathrm{B}_{b}(X)$. A simple truncation argument extends this property to arbitrary nonnegative Borel functions. 
(f) The image of $\Gamma_{*}:=R(\Gamma)$ through the arc-length reparametrization $R$ is relatively compact in $\mathrm{C}([0,1] ;(X, \tau))$ by Proposition 3.3. Since $\Gamma$ is the image of $\Gamma_{*}$ through the quotient map $\mathrm{q}$ : $\mathrm{C}([0,1] ; X)$ to $\mathrm{A}(X), \Gamma$ is relatively compact as well.

(g) Let us consider the compactification $(\hat{X}, \hat{\tau}, \hat{\mathrm{d}})$ as in Theorem 2.34 and claim (a), and let $\left[\gamma_{i}\right]$ be a net in $\Gamma$ with $\mu_{i}:=\nu_{\gamma_{i}}$. We also set $\hat{\mu}_{i}=\iota_{\sharp} \mu_{i}=\nu_{\hat{\gamma}_{i}}, \hat{\gamma}_{i}=\iota \circ \gamma_{i}$. It is not restrictive to assume $\gamma_{i}=R_{\gamma_{i}}$ so that $\gamma_{i}$ is uniformly Lipschitz. We can then apply Proposition 3.3 to the net $\hat{\gamma}_{i}$ in $\mathrm{C}([0,1] ; \hat{X})$ and find a subnet $j \mapsto h(j)$ and a limit curve $\gamma_{*} \in \operatorname{Lip}([0,1] ;(\hat{X}, \hat{\mathrm{d}}))$ such that $j \mapsto \hat{\gamma}_{h(j)}$ converges to $\gamma_{*}$ with respect to $\hat{\tau}_{\mathrm{C}}$. Since the total mass of $\mu_{i}=\ell\left(\gamma_{i}\right)$ remains bounded, we can also find a further subnet (still denoted by $h$ ) and a limit probability measure $\mu$ such that $\mu_{h(j)} \rightarrow \mu$. Since $\iota$ is continuous, we have $\hat{\mu}_{h(j)} \rightarrow \hat{\mu}=\iota_{\sharp} \mu$ with $m:=\mu(X)=\hat{\mu}(\hat{X})$.

If $m=0$ then $\ell\left(\gamma_{*}\right)=0$ so that $\gamma_{*}$ is constant and coincides with a point $\hat{x} \in \hat{X}$. Since the image of every curve $\gamma_{i}$ intersects the compact set $K$ we deduce that $\hat{x}=\iota(x)$ for some $x \in K$, so that $\gamma_{h(j)}$ converges to the constant curve $\gamma, \gamma(t) \equiv x$ w.r.t. $\tau_{\mathrm{C}}$ and $\left[\gamma_{h(j)}\right]$ converges to $[\gamma]$ in $\mathrm{A}(X)$.

If $m>0$, the uniform tightness condition shows that $\mu$ is concentrated on $\cup_{n \in \mathbb{N}} K_{1 / n}$ so that $\hat{\mu}(\hat{X} \backslash \iota(X))=0$. It follows that $\iota(X)$ is dense in $\operatorname{supp}(\hat{\mu})=\gamma_{*}([0,1])$. Since $\gamma_{*}$ is Lipschitz and $\iota(X)$ is complete, and thus d-closed, we conclude that $\gamma_{*}([0,1]) \subset \iota(X)$ and therefore $\gamma_{*}=\iota \circ \gamma$ for a curve $\gamma \in \operatorname{Lip}([0,1] ; X)$. We deduce that $j \mapsto \gamma_{h(j)}$ converges to $\gamma$ w.r.t. the compact-open topology $\tau_{\mathrm{C}}$ and therefore $\lim _{j \in J}\left[\gamma_{h(j)}\right]=[\gamma]$ w.r.t. $\tau_{\mathrm{A}}$.

\subsection{Notes}

$\S 3.1$ contains standard material on the compact-open topology (which is well adapted to deal with general topologies $\tau$ on $X)$ and its natural role in lifting the metric-topological structure of $(X, \tau, \mathrm{d})$ to the space $\left(\mathrm{C}([a, b] ; X), \tau_{\mathrm{C}}, \mathrm{d}_{\mathrm{C}}\right)$. The compactness result of Proposition 3.3 combines compactness w.r.t. $\tau$ and equicontinuity w.r.t. d, see also [7, Prop. 3.3.1].

$\S 3.2$ devotes some effort to construct a natural notion of invariance by parametrizations for arbitrary continuous curves. Since we did not assume rectifiability, the existence of a canonical arc-length parametrization is not guaranteed and one has to deal with a more general notion where arbitrary increasing, continuous and surjective change of variable are allowed (see [55] for a similar approach). Here the main properties are provided by Theorem 3.4. The construction of an extended metric-topological setting is presented in Proposition 3.6: although very natural, it requires a detailed proof. Everything becomes much simpler in the case of Example 2.9.

$\S 3.3$ combines the two previous sections to deal with continuous rectifiable arcs. The presentation here slightly differs from [2].

\section{Length and conformal distances}

\subsection{The length property}

To every extended metric space $(X, \mathrm{~d})$ it is possible to associate the length distance

$$
\mathrm{d}_{\ell}(x, y):=\inf \left\{\ell(\gamma): \gamma \in \operatorname{RA}(X), \gamma_{0}=x, \gamma_{1}=y\right\}
$$

$(X, \mathrm{~d})$ is a length space if $\mathrm{d}=\mathrm{d}_{\ell} .(X, \mathrm{~d})$ is a geodesic space if for every $x, y \in X$ with $\mathrm{d}(x, y)<\infty$ there exists an arc $\gamma \in \mathrm{RA}(X)$ connecting $x$ to $y$ with $\ell(\gamma)=\mathrm{d}(x, y)$.

It is not difficult to check that the classes of rectifiable arcs for $d$ and for $d_{\ell}$ coincide, as well as the corresponding notion of length and integral. 
When $(X, \mathrm{~d})$ is complete, it is possible to give an equivalent characterization of the length property in terms of the approximate mid-point property: every couple of points $x, y \in X$ with $\mathrm{d}(x, y)<\infty$ admits approximate midpoints

$$
\forall \theta>\frac{1}{2} \exists z_{\theta} \in X: \quad \mathrm{d}\left(x, z_{\theta}\right) \vee \mathrm{d}\left(z_{\theta}, y\right) \leq \theta \mathrm{d}(x, y) .
$$

By iterating the middle point construction, it is possible to show that for every $x, y \in X$ with $\mathrm{d}(x, y)<$ $\infty$ and for every $D>\mathrm{d}(x, y)$ there exists a map $\gamma: \mathbb{D} \rightarrow X$ defined on the set of dyadic points in $[0,1], \mathbb{D}:=\left\{k / 2^{n}: n, k \in \mathbb{N}, 0 \leq k \leq 2^{n}\right\}$, satisfying

$$
\mathrm{d}(\gamma(s), \gamma(t)) \leq D|t-s| \quad \text { for every } s, t \in \mathbb{D} .
$$

Thus, if $(X, \mathrm{~d})$ is complete the curve $\gamma$ admits a unique extension to a curve $\tilde{\gamma} \in \operatorname{BVC}([0,1] ; X)$ with $\ell(\tilde{\gamma}) \leq D$. Since $D>\mathrm{d}(x, y)$ is arbitrary, we conclude that $\mathrm{d}_{\ell}=\mathrm{d}$.

Notice that if $(X, \mathrm{~d})$ satisfies the approximate mid-point property then for every $x, y \in X, \varepsilon>0$, and $L>1$ there exists a sequence $\left(x_{n}\right)_{n=0}^{N} \subset X$ such that

$$
x_{0}=x, x_{N}=y, \quad \sup _{1 \leq n \leq N} \mathrm{~d}\left(x_{n-1}, x_{n}\right) \leq \varepsilon, \quad \sum_{n=1}^{N} \mathrm{~d}\left(x_{n-1}, x_{n}\right) \leq L \mathrm{~d}(x, y) .
$$

\subsection{Conformal distances}

More generally, let $g: X \rightarrow(0, \infty)$ be a continuous function satisfying

$$
m_{g}:=\inf _{X} g>0, \quad M_{g}:=\sup _{X} g<\infty .
$$

We can consider $g$ as a conformal metric density, inducing the length distance

$$
\mathrm{d}_{g}(x, y):=\inf \left\{\int_{\gamma} g: \gamma \in \operatorname{RA}(X), \gamma(0)=x, \gamma(1)=y\right\}
$$

It is clear that $\mathrm{d}_{g}$ is an extended distance and satisfies

$$
m_{g} \mathrm{~d}_{\ell}(x, y) \leq \mathrm{d}_{g}(x, y) \leq M_{g} \mathrm{~d}_{\ell}(x, y) \quad \text { for every } x, y \in X .
$$

By construction, $\mathrm{d}_{g}$ is a length distance, i.e. $\left(\mathrm{d}_{g}\right)_{\ell}=\mathrm{d}_{g}$; when $g \equiv 1$ we clearly have $\mathrm{d}_{g}=\mathrm{d}_{\ell}$.

We can introduce different inner approximations of $d_{g}$. The first one, $d_{g}^{\prime}$, arises by the the following procedure: first of all we set

$$
\beta(x, y):=(g(x) \vee g(y)) \mathrm{d}(x, y), \quad \beta_{i}(x, y):=(g(x) \vee g(y)) \mathbf{d}_{i}(x, y),
$$

where $\left(\mathrm{d}_{i}\right)_{i \in I}$ is a directed family of $\tau$-continuous bounded semidistances generating $\mathrm{d}$ by $\mathrm{d}(x, y)=$ $\lim _{i \in I} \mathrm{~d}_{i}(x, y)$ as in Lemma 2.4; for every $\varepsilon \in(0,+\infty]$ we first set

$$
\begin{aligned}
\mathrm{d}_{g, i, \varepsilon}(x, y):=\inf \{ & \sum_{n=1}^{N} \beta_{i}\left(x_{n-1}, x_{n}\right): N \in \mathbb{N},\left(x_{n}\right)_{n=0}^{N} \in X, \\
& \left.x_{0}=x, x_{N}=y, \mathrm{~d}_{i}\left(x_{n-1}, x_{n}\right)<\varepsilon\right\} \wedge\left(M_{g} \operatorname{supd}_{i}\right) .
\end{aligned}
$$


It is not difficult to check that $\mathrm{d}_{g, i, \varepsilon}$ is a bounded $\tau$-continuous semidistance with $\mathrm{d}_{g, i, \varepsilon}(x, y) \leq$ $\beta_{i}(x, y)$ whenever $\mathrm{d}_{i}(x, y)<\varepsilon$; moreover it is easy to check that if $0<\varepsilon<\varepsilon^{\prime}$ and $i \prec j$ we have $m_{g} \mathrm{~d}_{i} \leq \mathrm{d}_{g, i, \varepsilon^{\prime}} \leq \mathrm{d}_{g, j, \varepsilon} \leq M_{g} \mathrm{~d}_{j, \ell} \leq M_{g} \mathrm{~d}_{\ell}$. We need a more localized estimate involving the sets

$$
D_{i}(x, y):=\left\{\mathrm{d}_{i}(z, x) \vee \mathrm{d}_{i}(z, y) \leq \mathrm{d}_{i}(x, y)\right\}, \quad D(x, y):=\{\mathrm{d}(z, x) \vee \mathrm{d}(z, y) \leq \mathrm{d}(x, y)\},
$$

where $x, y \in X$. Notice that $D_{i}(x, y)$ and $D(x, y)$ are closed sets containing $x$ and $y$.

\section{Lemma 4.1.}

(a) For every $x, y \in X$ we have

$$
\mathrm{d}_{g, i, \varepsilon}(x, y) \geq \mathrm{d}_{i}(x, y) \inf _{D_{i}(x, y)} g
$$

(b) For every $z \in X, i \in I$ and $\varepsilon>0$ the map $h: x \mapsto \mathrm{d}_{g, i, \varepsilon}(x, z)$ belongs to $\operatorname{Lip}_{b}\left(X, \tau, \mathrm{d}_{i}\right)$ with

$$
\operatorname{lip}_{\mathrm{d}_{i}} h \leq g \text { in } X
$$

(c) If moreover $(X, \tau)$ is compact, then the infimum of $g$ on $D_{i}(x, y)$ and $D(x, y)$ is attained and

$$
\liminf _{i \in I} \min _{D_{i}(x, y)} g \geq \min _{D(x, y)} g \quad \text { for every } x, y \in X
$$

Proof. (a) Let $\left(x_{n}\right)_{n=0}^{N}$ be any sequence of points connecting $x$ to $y$ as in (4.9). If all the points $x_{n}$ belong to $D_{i}(x, y)$ then (4.11) immediately follows by the inequality

$$
\beta_{i}\left(x_{n-1}, x_{n}\right) \geq \mathrm{d}_{i}\left(x_{n-1}, x_{n}\right)\left(g\left(x_{n-1}\right) \vee g\left(x_{n}\right)\right) \geq \mathrm{d}_{i}\left(x_{n-1}, x_{n}\right) \inf _{D_{i}(x, y)} g .
$$

If not, there are indexes $n$ such that $\mathrm{d}_{i}\left(x_{n}, x\right) \vee \mathrm{d}_{i}\left(x_{n}, y\right)>\mathrm{d}_{i}(x, y)$. Just to fix ideas, let us suppose that the set of indexes $n \in\{1, \cdots, N-1\}$ such that $\mathrm{d}_{i}\left(x_{n}, x\right)>\mathrm{d}_{i}(x, y)$ is not empty and let us call $\bar{n}$ its minimum, so that $x_{n} \in D_{i}(x, y)$ if $0 \leq n<\bar{n}$. It follows that

$$
\begin{aligned}
\sum_{n=1}^{N} \beta_{i}\left(x_{n-1}, x_{n}\right) & \geq \sum_{n=1}^{\bar{n}} \beta_{i}\left(x_{n-1}, x_{n}\right) \stackrel{(4.14)}{\geq} \sum_{n=1}^{\bar{n}}\left(\inf _{D_{i}(x, y)} g\right) \mathrm{d}_{i}\left(x_{n-1}, x_{n}\right) \\
& \geq\left(\inf _{D_{i}(x, y)} g\right) \mathrm{d}_{i}\left(x, x_{\bar{n}}\right) \geq\left(\inf _{D_{i}(x, y)} g\right) \mathrm{d}_{i}(x, y) .
\end{aligned}
$$

A similar argument holds if the set of indexes $n \in\{1, \cdots, N-1\}$ such that $\mathrm{d}_{i}\left(x_{n}, y\right)>\mathrm{d}_{i}(x, y)$ is not empty: in this case one can select the greatest index.

(b) We first observe that for every $z \in X$ and $\varepsilon>0$ the map $h: x \mapsto \mathrm{d}_{g, i, \varepsilon}(z, x)$ belongs to $\operatorname{Lip}_{b}\left(X, \tau, \mathrm{d}_{i}\right)$ with Lipschitz constant bounded by $M_{g} \varepsilon^{-2}$. In fact the triangle inequality yields

$$
\left|\mathrm{d}_{g, i, \varepsilon}(z, x)-\mathrm{d}_{g, i, \varepsilon}(z, y)\right| \leq \mathrm{d}_{g, i, \varepsilon}(x, y)
$$

and

$$
\mathrm{d}_{g, i, \varepsilon}(x, y) \leq \begin{cases}M_{g} \mathrm{~d}_{i}(x, y) & \text { if } \mathrm{d}_{i}(x, y)<\varepsilon \\ \frac{M_{g} \operatorname{supd}_{i}}{\varepsilon} \mathrm{d}_{i}(x, y) & \text { if } \mathrm{d}_{i}(x, y) \geq \varepsilon\end{cases}
$$


On the other hand, for every $\bar{x} \in X$ the continuity of $\mathrm{d}_{i}$ and of $g$ ensures that there exists a neighborhood $U \in \mathscr{U}_{\bar{x}}$ such that $\mathrm{d}_{i}(\bar{x}, y)<\varepsilon / 2$ and $g(y) \leq g(\bar{x})+\varepsilon$ for every $y \in U$, so that

$$
\mathrm{d}_{g, i, \varepsilon}(x, y) \leq \beta_{i}(x, y) \leq \mathrm{d}_{i}(x, y) \sup _{U} g \leq \mathrm{d}_{i}(x, y)(g(\bar{x})+\varepsilon) \quad \text { for every } x, y \in U
$$

and therefore $\operatorname{Lip}\left(h, U, \mathrm{~d}_{i}\right) \leq(g(\bar{x})+\varepsilon), \operatorname{lip}_{\mathrm{d}_{i}} h(\bar{x}) \leq g(\bar{x})+\varepsilon$. Since $\varepsilon>0$ is arbitrary we conclude.

(c) Concerning (4.13), let $z_{i} \in D_{i}(x, y), i \in I$, be a minimizer for $g$ in $D_{i}(x, y)$, whose existence follows by the compactness of $(X, \tau)$ (and therefore of $\left.D_{i}(x, y)\right)$ and the continuity of $\mathrm{d}_{i}$. We can find a converging subnet $\alpha \mapsto i(\alpha), \alpha \in A$, such that $z_{i(\alpha)} \rightarrow z, g\left(z_{i(\alpha)}\right) \rightarrow g(z)=\liminf _{i \in I} g\left(z_{i}\right)$ and (recalling (2.25))

$$
\mathrm{d}(z, x) \vee \mathrm{d}(z, y) \leq \liminf _{\alpha \in A} \mathrm{~d}_{i(\alpha)}\left(z_{i(\alpha)}, x\right) \vee \mathrm{d}_{i(\alpha)}\left(z_{i(\alpha)}, y\right) \leq \liminf _{\alpha \in A} \mathrm{~d}_{i(\alpha)}(x, y)=\mathrm{d}(x, y),
$$

so that $z \in D(x, y)$. It follows that

$$
\liminf _{i \in I} \min _{D_{i}(x, y)} g=\liminf _{i \in I} g\left(z_{i}\right)=g(z) \geq \min _{D(x, y)} g .
$$

We then define

$$
\mathrm{d}_{g}^{\prime}(x, y):=\lim _{\varepsilon \downarrow 0, i \in I} \mathrm{~d}_{g, i, \varepsilon}(x, y)=\sup _{\varepsilon>0, i \in I} \mathrm{~d}_{g, i, \varepsilon}(x, y) .
$$

Different approximations of $\mathrm{d}_{g}$ are provided by the formula

$$
\begin{gathered}
\mathrm{d}_{g}^{\prime \prime}(x, y):=\sup \{|f(x)-f(y)|: \exists i \in I \text { such that } \\
\left.f \in \operatorname{Lip}_{b}\left(X, \tau, \mathrm{d}_{i}\right) \text { and } \operatorname{lip}_{\mathrm{d}_{i}} f \leq g \text { in } X\right\} \\
\mathrm{d}_{g}^{\prime \prime \prime}(x, y):=\sup \{|f(x)-f(y)|: \\
\left.f \in \operatorname{Lip}_{b}(X, \tau, \mathrm{d}) \text { and } \operatorname{lip}_{\mathrm{d}} f \leq g \text { in } X\right\} .
\end{gathered}
$$

When $g \equiv 1$ we will also write $\mathrm{d}_{\ell}^{\prime}:=\mathrm{d}_{1}^{\prime}, \mathrm{d}_{\ell}^{\prime \prime}:=\mathrm{d}_{1}^{\prime \prime}, \mathrm{d}_{\ell}^{\prime \prime \prime}:=\mathrm{d}_{1}^{\prime \prime \prime}$. In the next Lemma we collect a few results concerning these distances.

Theorem 4.2. (a) If $(X, \tau, \mathrm{d})$ is an extended metric-topological space, then also $\left(X, \tau, \mathrm{d}_{g}^{\prime}\right),\left(X, \tau, \mathrm{d}_{g}^{\prime \prime}\right)$ and $\left(X, \tau, \mathrm{d}_{g}^{\prime \prime \prime}\right)$ are extended metric-topological space and we have for every $x, y \in X$

$$
\mathrm{d}_{g}^{\prime}(x, y) \leq \mathrm{d}_{g}^{\prime \prime}(x, y) \leq \mathrm{d}_{g}^{\prime \prime \prime}(x, y) \leq \mathrm{d}_{g}(x, y) .
$$

(b)

$$
\left(\mathrm{d}_{g}^{\prime}\right)_{\ell}=\left(\mathrm{d}_{g}^{\prime \prime}\right)_{\ell}=\left(\mathrm{d}_{g}^{\prime \prime \prime}\right)_{\ell}=\mathrm{d}_{g}
$$

(c) If $(X, \tau)$ is compact then $\mathrm{d}_{g}^{\prime}=\mathrm{d}_{g}^{\prime \prime}=\mathrm{d}_{g}^{\prime \prime \prime}=\mathrm{d}_{g}$. In particular, $\left(X, \tau, \mathrm{d}_{g}\right)$ is an extended metrictopological space and $\left(X, \mathrm{~d}_{g}\right)$ is a geodesic space.

Proof. (a) The fact that we are dealing with extended metric-topological spaces is clear from the construction.

The first inequality $\mathrm{d}_{g}^{\prime} \leq \mathrm{d}_{g}^{\prime \prime}$ in (4.18) follows immediately by Lemma 4.1(b). 

set.

The inequality $\mathrm{d}_{g}^{\prime \prime} \leq \mathrm{d}_{g}^{\prime \prime \prime}$ is obvious since the latter is obtained by taking the supremum on a bigger

The last inequality $\mathrm{d}_{g}^{\prime \prime \prime} \leq \mathrm{d}_{g}$ easily follows since for every $\gamma \in \operatorname{BVC}([0,1] ; X)$ and every map $f \in \operatorname{Lip}(X, \tau, \mathrm{d})$ with $\operatorname{lip}_{\mathrm{d}} f \leq g$, the composition $\mathrm{f}:=f \circ R_{\gamma}$ is Lipschitz with

$$
\left|\mathrm{f}^{\prime}(t)\right| \leq \ell(\gamma) \operatorname{lip}_{\mathrm{d}} f\left(R_{\gamma}(t)\right) \leq \ell(\gamma) g\left(R_{\gamma}(t)\right) \quad \mathscr{L}^{1} \text {-a.e. in }[0,1] .
$$

An integration in the interval $[0,1]$ yields

$$
|f(\gamma(1))-f(\gamma(0))| \leq \int_{\gamma} g
$$

and a further minimization w.r.t. all the curves $\gamma$ connecting $x=\gamma(0)$ and $y=\gamma(1)$ yields for every $f$ satisfying (4.17)

$$
|f(y)-f(x)| \leq \mathrm{d}_{g}(x, y) .
$$

Taking the supremum w.r.t. $f$ we conclude.

(b) Since $\mathrm{d}_{g}$ is a length distance, $\left(\mathrm{d}_{g}^{\prime}\right)_{\ell} \leq \mathrm{d}_{g}$, so that it is sufficient to prove the converse inequality. Let $x, y \in X$ with $\left(\mathrm{d}_{g}^{\prime}\right)_{\ell}(x, y)<D$; we can find $\gamma \in \operatorname{Lip}([0,1] ;(X, \mathrm{~d}))$ with $\gamma(0)=x, \gamma(1)=y$, and $\mathrm{d}_{g}^{\prime}(\gamma(s), \gamma(t)) \leq D|s-t|$ for every $0 \leq s<t \leq 1$. We want to show

$$
I:=\int_{\gamma} g \leq D
$$

By (4.7) $\mathrm{d}(\gamma(s), \gamma(t)) \leq m_{g}^{-1} D|t-s|$ so that $\gamma$ is also d-Lipschitz. The map $g \circ \gamma$ is uniformly continuous as well. A standard compactness argument shows that for every $\varepsilon>0$ there exists $\delta>0$ such that

$$
\inf \{g(z): z \in X, \mathrm{~d}(z, x) \leq \delta\} \geq g(x)-\varepsilon \text { for every } x \in \gamma([0,1]) .
$$

By (3.51) for every $I_{1}<I$ and $\varepsilon>0$ we can find a subdivision $\left(t_{n}\right)_{n=0}^{N}$ of $[0,1]$ such that

$$
\sum_{n=1}^{N}\left(\inf _{\left[t_{n-1}, t_{n}\right]} g \circ R_{\gamma}\right) \mathrm{d}\left(R_{\gamma}\left(t_{n-1}\right), R_{\gamma}\left(t_{n}\right)\right)>I_{1}, \quad D\left|t_{n}-t_{n-1}\right| \leq(\delta \wedge \varepsilon) m_{g}
$$

so that, in particular, $\mathrm{d}\left(R_{\gamma}\left(t_{n-1}\right), R_{\gamma}\left(t_{n}\right)\right) \leq \varepsilon$. We set

$$
m_{n}:=\min _{\left[t_{n-1}, t_{n}\right]} g, \quad m_{i, n}:=\inf _{D_{i}\left(R_{\gamma}\left(t_{n-1}\right), R_{\gamma}\left(t_{n}\right)\right)} g, \quad i \in I, 1 \leq n \leq N .
$$

We can then find $i_{0} \in I$ such that for every $i \succeq i_{0}$

$$
\sum_{n=1}^{N} m_{n} \mathrm{~d}_{i}\left(R_{\gamma}\left(t_{n-1}\right), R_{\gamma}\left(t_{n}\right)\right) \geq I_{1}
$$


Applying (4.11) we obtain

$$
\begin{aligned}
I_{1} & \leq \sum_{n=1}^{N} m_{i, n} \mathrm{~d}_{i}\left(R_{\gamma}\left(t_{n-1}\right), R_{\gamma}\left(t_{n}\right)\right)+\sum_{n=1}^{N}\left(m_{n}-m_{i, n}\right) \mathrm{d}_{i}\left(R_{\gamma}\left(t_{n-1}\right), R_{\gamma}\left(t_{n}\right)\right) \\
& \leq \sum_{n=1}^{N} m_{i, n} \mathrm{~d}_{i}\left(R_{\gamma}\left(t_{n-1}\right), R_{\gamma}\left(t_{n}\right)\right)+m_{g}^{-1} D \sup _{1 \leq n \leq N}\left(m_{n}-m_{i, n}\right) \\
& \leq \sum_{n=1}^{N} \mathrm{~d}_{g, i, \varepsilon}\left(R_{\gamma}\left(t_{n-1}\right), R_{\gamma}\left(t_{n}\right)\right)+m_{g}^{-1} D \sup _{1 \leq n \leq N}\left(m_{n}-m_{i, n}\right) \\
& \leq D+m_{g}^{-1} D \sup _{1 \leq n \leq N}\left(m_{n}-m_{i, n}\right)
\end{aligned}
$$

We can now pass to the limit w.r.t. $i \in I$, observing that by (4.13) and (4.24)

$$
\liminf _{i \in I} m_{i, n} \geq \min _{D\left(R_{\gamma}\left(t_{n-1}\right), R_{\gamma}\left(t_{n}\right)\right)} g \geq g\left(R_{\gamma}\left(t_{n}\right)\right)-\varepsilon \geq m_{n}-\varepsilon
$$

since $\mathrm{d}\left(R_{\gamma}\left(t_{n-1}\right), R_{\gamma}\left(t_{n}\right)\right) \leq \delta$. It follows that

$$
I_{1} \leq D+m_{g}^{-1} D \varepsilon
$$

since $I_{1}<I$ and $\varepsilon>0$ are arbitrary, we conclude.

(c) We will show that $\left(X, \mathrm{~d}_{g}^{\prime}\right)$ is a geodesic space. Since $(X, \tau)$ is compact, it is sufficient to prove that $\left(X, \mathrm{~d}_{g}^{\prime}\right)$ satisfies the approximate mid-point property. In particular $\left(\mathrm{d}_{g}^{\prime}\right)_{\ell}=\mathrm{d}_{g}^{\prime}$ and the claim will follow by the previous point (b).

Let us fix couple $x, y \in X$ with $2 D:=\mathrm{d}_{g}^{\prime}(x, y)<\infty$. By definition, for every $\varepsilon>0$ we can find $\eta_{0}>0$ with $\left(M_{g} \vee 1\right) \eta_{0}<\varepsilon$ and $i_{0} \in I$ such that $2 D-\varepsilon<\mathrm{d}_{g, i, \eta}(x, y) \leq 2 D$ for every $0<\eta \leq \eta_{0}$ and $i \succeq i_{0}$. Therefore, we find points $\left(x_{n}\right)_{n=0}^{N} \in X$ (depending on $i, \eta$ ) such that $\mathrm{d}_{i}\left(x_{n-1}, x_{n}\right)<\eta$ and $2 D-\varepsilon<\sum_{n=1}^{N} \beta_{i}\left(x_{n-1}, x_{n}\right) \leq \mathrm{d}_{g, i, \eta}(x, y)+\varepsilon \leq 2 D+\varepsilon$. If $k_{0}=\max \{k \leq$ $\left.N: \sum_{n=1}^{k} \beta_{i}\left(x_{n-1}, x_{n}\right) \leq D\right\}$ and $z_{i, \eta}:=x_{k_{0}+1}$, we clearly have

$$
\begin{aligned}
\mathrm{d}_{g, i, \eta}\left(x, z_{i, \eta}\right) & \leq D+\beta_{i}\left(x_{k_{0}}, x_{k_{0}+1}\right) \leq D+M_{g} \eta \leq D+\varepsilon \\
\mathrm{d}_{g, i, \eta}\left(y, z_{i, \eta}\right) & \leq \sum_{n=k_{0}+1}^{N} \beta_{i}\left(x_{n-1}, x_{n}\right)=\sum_{n=1}^{N} \beta_{i}\left(x_{n-1}, x_{n}\right)-\sum_{n=1}^{k_{0}+1} \beta_{i}\left(x_{n-1}, x_{n}\right) \\
& \leq 2 D+\varepsilon-D \leq D+\varepsilon .
\end{aligned}
$$

Let now $(h, k): J \rightarrow\left\{i \in I: i \succeq i_{0}\right\} \times\left(0, \eta_{0}\right)$ be a monotone subnet such that $z_{(h(j), k(j))}$ converges to $z \in X$. Since $(i, \eta) \mapsto \mathrm{d}_{g, i, \eta}$ is monotone, for every $i \in I$ and $\eta>0$ we have

$$
\begin{aligned}
& \mathrm{d}_{g, i, \eta}(x, z)=\lim _{j \in J} \mathrm{~d}_{g, i, \eta}\left(x, z_{h(j), k(j)}\right) \leq \limsup _{j \in J} \mathrm{~d}_{g, h(j), k(j)}\left(x, z_{h(j), k(j)}\right) \leq D+\varepsilon, \\
& \mathrm{d}_{g, i, \eta}(y, z)=\lim _{j \in J} \mathrm{~d}_{g, i, \eta}\left(y, z_{h(j), k(j)}\right) \leq \limsup _{j \in J} \mathrm{~d}_{g, h(j), k(j)}\left(y, z_{h(j), k(j)}\right) \leq D+\varepsilon .
\end{aligned}
$$

Taking the supremum w.r.t. $i \in I$ and $\eta>0$ we eventually get

$$
\mathrm{d}_{g}^{\prime}(x, z) \leq D+\varepsilon, \quad \mathrm{d}_{g}^{\prime}(y, z) \leq D+\varepsilon
$$

so that $z$ is an $\varepsilon$-approximate midpoint between $x$ and $y$.

Remark 4.3. Notice that when $\mathrm{d}_{g}$ is $\tau$-continuous, then also $\mathrm{d}$ is $\tau$-continuous and $\mathrm{d}_{g}=\mathrm{d}_{g}^{\prime \prime}=\mathrm{d}_{g}^{\prime \prime \prime}$. In this case $\left(X, \tau, \mathrm{d}_{g}\right)$ is an extended metric-topological space. 


\subsection{Duality for Kantorovich-Rubinstein cost functionals induced by conformal dis- tances}

We apply Theorem 4.2 to obtain a useful dual representation for Kantorovich-Rubinstein distances.

Proposition 4.4. Let us suppose that the extended distances $\mathrm{d}_{g}$ and $\mathrm{d}_{g}^{\prime}$ defined by (4.6) and (4.15) coincide (in particular when $(X, \tau)$ is compact) and let $\mathrm{K}_{\mathrm{d}_{g}}$ be the Kantorovich functional induced by $\mathrm{d}_{g}$. Then for every $\mu_{0}, \mu_{1} \in \mathcal{M}_{+}(X)$ with the same mass

$$
\begin{aligned}
\mathrm{K}_{\mathrm{d}_{g}}\left(\mu_{0}, \mu_{1}\right)= & \sup \left\{\int \phi_{0} \mathrm{~d} \mu_{0}-\int \phi_{1} \mathrm{~d} \mu_{1}: \phi_{i} \in \mathrm{C}_{b}(X, \tau),\right. \\
& \left.\phi_{0}\left(x_{0}\right)-\phi_{1}\left(x_{1}\right) \leq \mathrm{d}_{g}\left(x_{0}, x_{1}\right) \quad \text { for every } x_{0}, x_{1} \in X\right\} \\
= & \sup \left\{\int \phi \mathrm{d}\left(\mu_{0}-\mu_{1}\right): \phi \in \operatorname{Lip}_{b}(X, \tau, \mathrm{d}), \operatorname{lip}_{\mathrm{d}} \phi \leq g\right\} .
\end{aligned}
$$

Proof. (4.29) is a particular case of (2.45) for the extended metric-topological space $\left(X, \tau, \mathrm{d}_{g}\right)$, thanks to Theorem 4.2(c).

Concerning (4.30), we can first observe that the right hand side is dominated by $\mathrm{K}_{d g}$ since every function $\phi \in \operatorname{Lip}_{b}(X, \tau, \mathrm{d})$ with $\operatorname{lip}_{\mathrm{d}} \phi \leq g$ belongs to $\operatorname{Lip}_{b, 1}\left(X, \tau, \mathrm{d}_{g}\right)$ thanks to (4.18) and the very definition of $\mathrm{d}_{g}^{\prime \prime \prime}$ given by (4.17).

On the other hand, we know by (4.18) that $\mathrm{d}_{g}=\mathrm{d}_{g}^{\prime}$ so that the collection $\left(\mathrm{d}_{g, i, \varepsilon}\right)_{i \in I, \varepsilon>0}$ is a direct set of continuous and bounded semidistances giving (4.15). We can then apply (2.44) obtaining

$$
\mathrm{K}_{d_{g}}\left(\mu_{0}, \mu_{1}\right)=\lim _{i \in I, \varepsilon \downarrow 0} \mathrm{~K}_{d_{g, i, \varepsilon}}\left(\mu_{0}, \mu_{1}\right),
$$

so that (2.43) yields

$$
\mathrm{K}_{d_{g}}\left(\mu_{0}, \mu_{1}\right)=\sup \left\{\int \phi \mathrm{d}\left(\mu_{0}-\mu_{1}\right): \phi \in \operatorname{Lip}_{b, 1}\left(X, \tau, \mathrm{d}_{g, i, \varepsilon}\right), i \in I, \varepsilon>0\right\} .
$$

On the other hand, using (4.12) one immediately sees that

$$
\phi \in \operatorname{Lip}_{b, 1}\left(X, \tau, \mathrm{d}_{g, i, \varepsilon}\right) \quad \Rightarrow \quad \operatorname{lip}_{\mathrm{d}} \phi \leq \operatorname{lip}_{\mathrm{d}_{i}} \phi \leq g
$$

\subsection{Notes}

$\S \mathbf{4 . 1}$ is standard, see e.g. [22]

$\S 4.2$ will play a crucial role in the proof of the identification Theorem for metric Sobolev spaces of Section 11. One of the main point here is that even in standard metric spaces the length-conformal construction may easily lead to extended distances. Theorem 4.2 shows that at least in the compact case we can recover the lengthconformal distances by inner approximation with $\tau$-continuous Lipschitz functions. Such kind of constructions and dual representations by local Lipschitz bounds are typical in the study of local properties of Dirichlet forms, see e.g. $[16,61,60]$.

$\S 4.3$ contains the natural extension to the Kantorovich distance of the dual characterization $\mathrm{d}_{g}=\mathrm{d}_{g}^{\prime \prime \prime}$; it will play a crucial role in $\S 11.2$. Notice that if $\mathrm{d}_{g}$ is continuous Proposition 4.4 could be proved by a more direct argument based on the identity $\mathrm{d}_{g}=\mathrm{d}_{g}^{\prime \prime \prime}$ and on the classic representation (2.46) for $\mathrm{d}_{g}$. 


\section{Part II}

\section{The Cheeger energy}

In all this part we will always refer to this basic setting:

Assumption. Let $\mathbb{X}=(X, \tau, \mathrm{d}, \mathfrak{m})$ be an extended metric-topological measure space as in $\S 2.2$ and let $\mathscr{A} \subset \operatorname{Lip}_{b}(X, \tau, \mathrm{d})$ be a compatible algebra of functions, according to Definition 2.17. For $f \in \operatorname{Lip}_{b}(X, \tau, \mathrm{d}) \operatorname{lip} f$ will always refer to the asymptotic Lipschitz constant lip $\operatorname{d}_{\mathrm{d}} f$ defined in $\S 2.5$. We fix an exponent $p \in(1, \infty)$

\section{The strongest form of the Cheeger energy}

Let us first define the notion of Cheeger energy $\mathrm{CE}_{p, \mathscr{A}}$ associated to $(\mathbb{X}, \mathscr{A})$.

Definition 5.1 (Cheeger energy). For every $\kappa \geq 0$ and $p \in(1, \infty)$ we define the "pre-Cheeger" energy functionals

$$
\mathrm{pCE}_{p}(f):=\int_{X}(\operatorname{lip} f(x))^{p} \mathrm{dm} \quad \text { for every } f \in \operatorname{Lip}_{b}(X, \tau, \mathrm{d}) .
$$

The $L^{p}$-lower semicontinuous envelope of the restriction to $\mathscr{A}$ of $\mathrm{pCE}_{p, \kappa}$ is the "strong" Cheeger energy

$$
\mathrm{CE}_{p, \mathscr{A}}(f):=\inf \left\{\liminf _{n \rightarrow \infty} \int_{X}\left(\operatorname{lip} f_{n}\right)^{p} \mathrm{dm}: f_{n} \in \mathscr{A}, f_{n} \rightarrow f \text { in } L^{p}(X, \mathfrak{m})\right\} .
$$

When $\mathscr{A}=\operatorname{Lip}_{b}(X, \tau, \mathrm{d})$ we will simply write $\mathrm{CE}_{p}(f)$.

Remark 5.2 (The notation CE). We used the symbol CE instead of Ch (introduced by [9]) in the previous definition to stress three differences:

- the dependence on the strongest $\operatorname{lip}_{d} f$ instead of $|\mathrm{D} f|$,

- the restriction to functions in the algebra $\mathscr{A} \subset \operatorname{Lip}_{b}(X, \tau, \mathrm{d})$,

- the factor 1 instead of $1 / p$ in front of the energy integral.

It is not difficult to check that $\mathrm{CE}_{p}: L^{p}(X, \mathfrak{m}) \rightarrow[0,+\infty]$ is a convex, lower semicontinuous and $p$ homogeneous functional; it is the greatest $L^{p}$-lower semicontinuous functional "dominated" by $\mathrm{pCE}_{p}$ (extended to $+\infty$ whenever a function does not belong to $\mathscr{A}$ ).

Definition 5.3. We denote by $H^{1, p}(\mathbb{X}, \mathscr{A})$ the subset of $L^{p}(X, \mathfrak{m})$ whose elements $f$ have finite Cheeger energy $\mathrm{CE}_{p, \mathscr{A}}(f)<\infty$ : it is a Banach space with norm

$$
\|f\|_{H^{1, p}(\mathbb{X}, \mathscr{A})}:=\left(\mathrm{CE}_{p, \mathscr{A}}(f)+\|f\|_{L^{p}(X, \mathfrak{m})}^{p}\right)^{1 / p} .
$$

When $\mathscr{A}=\operatorname{Lip}_{b}(X, \tau, \mathrm{d})$ we will simply write $H^{1, p}(\mathbb{X})$. 
Remark 5.4 $\left(H^{1, p}(\mathbb{X}, \mathscr{A})\right.$ as Gagliardo completion [34]). Recall that if $\left(A,\|\cdot\|_{A}\right)$ is a normed vector space continuously imbedded in a Banach space $\left(B,\|\cdot\|_{B}\right)$, the Gagliardo completion $A^{B, c}$ is the Banach space defined by

$$
A^{B, c}:=\left\{b \in B: \exists\left(a_{n}\right)_{n} \subset A, \lim _{n \rightarrow \infty}\left\|a_{n}-b\right\|_{B}=0, \sup _{n}\left\|a_{n}\right\|_{A}<\infty\right\}
$$

with norm

$$
\|b\|_{A^{B, c}}:=\inf \left\{\liminf _{n \rightarrow \infty}\left\|a_{n}\right\|_{A}: a_{n} \in A, \lim _{n \rightarrow \infty}\left\|a_{n}-b\right\|_{B}=0\right\} .
$$

When $\operatorname{supp}(\mathfrak{m})=X$, we can identify $\mathscr{A}$ with a vector space $A$ with the norm induced by $\mathrm{pCE}_{p}$ imbedded in $B:=L^{p}(X, \mathfrak{m})$; it is immediate to check that $H^{1, p}(\mathbb{X}, \mathscr{A})$ coincides with the Gagliardo completion of $A$ in $B$.

Notice that when $\mathfrak{m}$ has not full support, two different elements $f_{1}, f_{2} \in \mathscr{A}$ may give rise to the same equivalence class in $L^{p}(X, \mathfrak{m})$. In this case, $\mathrm{CE}_{p}$ can be equivalently defined starting from the functional

$$
\widetilde{\mathrm{pCE}}_{p}(f):=\inf \left\{\operatorname{pCE}_{p}(\tilde{f}): \tilde{f} \in \mathscr{A}, \tilde{f}=f \mathfrak{m} \text {-a.e. }\right\},
$$

defined on the quotient space

$$
\tilde{\mathscr{A}}:=\mathscr{A} / \sim_{\mathfrak{m}}, \quad f_{1} \sim_{\mathfrak{m}} f_{2} \text { if } f_{1}=f_{2} \mathfrak{m} \text {-a.e. }
$$

\subsection{Relaxed gradients and local representation of the Cheeger energy}

The Cheeger energy $\mathrm{CE}_{p, \mathscr{A}}$ admits an integral representation in terms of the minimal relaxed gradient $|\mathrm{D} f|_{\star, \mathscr{A}}$ : we collect here a series of useful results, which mainly follow by properties (2.51a-e) of Lemma 2.16 arguing as in [9, Lemma 4.3, 4.4, Prop. 4.8]. Here we have also to take into account the role of the algebra $\mathscr{A}$.

Definition 5.5 (Relaxed gradients). We say that $G \in L^{p}(X, \mathfrak{m})$ is a $(p, \mathscr{A})$-relaxed gradient of $f \in$ $L^{p}(X, \mathfrak{m})$ if there exist functions $f_{n} \in \mathscr{A}$ such that:

(a) $f_{n} \rightarrow f$ in $L^{p}(X, \mathfrak{m})$ and $\operatorname{lip} f_{n}$ weakly converge to $\tilde{G}$ in $L^{p}(X, \mathfrak{m})$;

(b) $\tilde{G} \leq G \mathfrak{m}$-a.e. in $X$.

We say that $G$ is the minimal $(p, \mathscr{A})$-relaxed gradient of $f$ if its $L^{p}(X, \mathfrak{m})$ norm is minimal among relaxed gradients. We shall denote by $|\mathrm{D} f|_{\star, \mathscr{A}}$ the minimal relaxed gradient. As usual, we omit the explicit dependence on $\mathscr{A}$ when $\mathscr{A}=\operatorname{Lip}_{b}(X, \tau, \mathrm{d})$.

Thanks to (2.51a) and the reflexivity of $L^{p}(X, \mathfrak{m})$ one can easily check that

$$
S:=\left\{(f, G) \in L^{p}(X, \mathfrak{m}) \times L^{p}(X, \mathfrak{m}): G \text { is a }(p, \mathscr{A}) \text {-relaxed gradient of } f\right\}
$$

is convex. Its closure follows by the following lemma, which also shows that it is possible to obtain the minimal relaxed gradient as strong limit in $L^{p}$.

Lemma 5.6 (Closure and strong approximation of the minimal relaxed gradient).

(a) If $(f, G) \in S$ then there exist functions $f_{n} \in \mathscr{A}, G_{n} \in \operatorname{Lip}_{b}(X, \tau, \mathrm{d})\left(G_{n} \in \mathscr{A}\right.$ if $\mathscr{A}$ is adapted) strongly converging to $f, \tilde{G}$ in $L^{p}(X, \mathfrak{m})$ with $\operatorname{lip} f_{n} \leq G_{n}$ and $\tilde{G} \leq G$. 
(b) $S$ is weakly closed in $L^{p}(X, \mathfrak{m}) \times L^{p}(X, \mathfrak{m})$.

(c) The collection of all the relaxed gradients of $f$ is closed in $L^{p}(X, \mathfrak{m})$; if it is not empty, it contains a unique element of minimal norm and there exist functions $f_{n} \in \mathscr{A}, G_{n} \in \operatorname{Lip}_{b}(X, \tau, \mathrm{d})$ $\left(G_{n} \in \mathscr{A}\right.$ if $\mathscr{A}$ is adapted) such that $G_{n} \geq \operatorname{lip} f_{n}$ and

$$
f_{n} \rightarrow f, \quad G_{n} \rightarrow|\mathrm{D} f|_{\star, \mathscr{A}}, \quad \operatorname{lip} f_{n} \rightarrow|\mathrm{D} f|_{\star, \mathscr{A}} \quad \text { strongly in } L^{p}(X, \mathfrak{m}) .
$$

Proof. (a) Since $G$ is a relaxed gradient, we can find functions $h_{i} \in \mathscr{A}$ such that $h_{i} \rightarrow f$ in $L^{p}(X, \mathfrak{m})$ and lip $h_{i}$ weakly converges to $\tilde{G} \leq G$ in $L^{p}(X, \mathfrak{m})$. Since lip $h_{i}$ are bounded, nonnegative and upper semicontinuous, by Corollary 2.38 we can find functions $g_{i} \in \mathscr{A}(\mathbb{X})\left(g_{i} \in \mathscr{A}\right.$ if $\mathscr{A}$ is adapted) such that $g_{i} \geq \operatorname{lip} h_{i}$ and $\left\|g_{i}-\operatorname{lip} h_{i}\right\|_{L^{p}(X, \mathfrak{m})} \leq 2^{-i}$ so that $\tilde{G}$ is also the weak limit of $g_{i}$ in $L^{p}(X, \mathfrak{m})$. By Mazur's lemma we can find a sequence of convex combinations $G_{n}$ of $g_{i}$ (thus belonging to $\mathscr{A}$ ), starting from an index $i(n) \rightarrow \infty$, strongly convergent to $\tilde{G}$ in $L^{p}(X, \mathfrak{m})$; the corresponding convex combinations of $h_{i}$, that we shall denote by $f_{n}$, still belong to $\mathscr{A}$, converge in $L^{p}(X, \mathfrak{m})$ to $f$ and $\operatorname{lip} f_{n}$ is bounded from above by $G_{n}$, thanks to (2.51a).

(b) Let us prove now the weak closure in $L^{p}(X, \mathfrak{m}) \times L^{p}(X, \mathfrak{m})$ of $S$. Since $S$ is convex, it is sufficient to prove that $S$ is strongly closed. If $S \ni\left(f^{i}, G^{i}\right) \rightarrow(f, G)$ strongly in $L^{p}(X, \mathfrak{m}) \times L^{p}(X, \mathfrak{m})$, we can find sequences of functions $\left(f_{n}^{i}\right)_{n} \in \mathscr{A}$ and of nonnegative functions $\left(G_{n}^{i}\right)_{n} \in L^{p}(X, \mathfrak{m})$ such that

$$
f_{n}^{i} \stackrel{n \rightarrow \infty}{\longrightarrow} f^{i}, \quad G_{n}^{i} \stackrel{n \rightarrow \infty}{\longrightarrow} \tilde{G}^{i} \text { strongly in } L^{p}(X, \mathfrak{m}), \quad \operatorname{lip} f_{n}^{i} \leq G_{n}^{i}, \quad \tilde{G}^{i} \leq G^{i} .
$$

Possibly extracting a suitable subsequence, we can assume that $\tilde{G}^{i} \rightarrow \tilde{G}$ weakly in $L^{p}(X, \mathfrak{m})$ with $\tilde{G} \leq G$; by a standard diagonal argument we can find an increasing sequence $i \mapsto n(i)$ such that $f_{n(i)}^{i} \rightarrow f, G_{n(i)}^{i} \rightarrow \tilde{G}$ in $L^{p}(X, \mathfrak{m})$ and $\operatorname{lip} f_{n(i)}^{i}$ is bounded in $L^{p}(X, \mathfrak{m})$. By the reflexivity of $L^{p}(X, \mathfrak{m})$ we can also assume, possibly extracting a further subsequence, that $\operatorname{lip} f_{n(i)}^{i} \rightarrow H$. It follows that $H \leq \tilde{G} \leq G$ so that $G$ is a relaxed gradient for $f$.

(c) The closure of the collection of the relaxed gradients of $f$ follows by the previous claim. Since the $L^{p}$-norm is strictly convex, if it is not empty it contains a unique element of minimal norm.

Let us consider now the minimal relaxed gradient $G:=|\mathrm{D} f|_{\star, \mathscr{A}}$ and let $f_{n}, G_{n}$ be sequences in $L^{p}(X, \mathfrak{m})$ as in the first part of the present Lemma. Since lip $f_{n}$ is uniformly bounded in $L^{p}(X, \mathfrak{m})$ it is not restrictive to assume that it is weakly convergent to some limit $H \in L^{p}(X, \mathfrak{m})$ with $0 \leq H \leq$ $\tilde{G} \leq G$. This implies at once that $H=\tilde{G}=G$ and $\operatorname{lip} f_{n}$ weakly converges to $|\mathrm{D} f|_{\star, \mathscr{A}}$ (because any limit point in the weak topology of $\operatorname{lip} f_{n}$ is a relaxed gradient with minimal norm) and that the convergence is strong, since

$$
\limsup _{n \rightarrow \infty} \int_{X}\left(\operatorname{lip} f_{n}\right)^{p} \mathrm{~d} \mathfrak{m} \leq \limsup _{n \rightarrow \infty} \int_{X} G_{n}^{p} \mathrm{~d} \mathfrak{m}=\int_{X} G^{p} \mathrm{~d} \mathfrak{m}=\int_{X} H^{p} \mathrm{~d} \mathfrak{m} .
$$

Corollary 5.7 (Representation of the Cheeger energy). A function $f \in L^{p}(X, \mathfrak{m})$ belongs to $H^{1, p}(\mathbb{X}, \mathscr{A})$ if and only if it admits a p-relaxed gradients. In this case

$$
\mathrm{CE}_{p, \mathscr{A}}(f)=\int_{X}|\mathrm{D} f|_{\star, \mathscr{A}}^{p} \mathrm{dm} .
$$

Remark 5.8 (Dependence of $|\mathrm{D} f|_{\star}$ with respect to $p$ ). Notice that $|\mathrm{D} f|_{\star}$ may depend on $p$, even for Lipschitz functions, see e.g. [8]. Since in these notes we will keep the exponent $p$ fixed, we will omit to denote this dependence in the notation for $|\mathrm{D} f|_{\star}$. 
We want to show now that if $f \in H^{1, p}(\mathbb{X}, \mathscr{A})$ satisfies the uniform bound $a \leq f \leq b \mathfrak{m}$-a.e. in $X$, then there exists a sequence $f_{n} \in \mathscr{A}$ satisfying (5.9) and the same uniform bounds of $f$. This result is trivial if $\mathscr{A}$ is the algebra of bounded Lipschitz functions, since truncations operates on $\mathscr{A}$. In the general case we use the approximated truncation polynomials of Corollary 2.24 .

Corollary 5.9. Let $f \in H^{1, p}(\mathbb{X}, \mathscr{A})$ be satisfying the uniform bounds $\alpha \leq f \leq \beta \mathfrak{m}$-a.e. in $X$. Then there exists a sequence $\left(f_{n}\right) \subset \mathscr{A}$ satisfying (5.9) such that $\alpha \leq f_{n} \leq \beta$ in $X$ for every $n \in \mathbb{N}$.

Proof. Let $\left(f_{n}\right)_{n \in \mathbb{N}}$ be a sequence in $\mathscr{A}$ as in (5.9). Since functions in $\mathscr{A}$ are bounded, we can find a sequence $c_{n}>0$ such that $f_{n}(X) \subset\left[-c_{n}, c_{n}\right]$. Let us choose a vanishing sequence $\varepsilon_{n} \downarrow 0$ and consider the truncation polynomials $P_{n}=P_{\varepsilon_{n}}^{c_{n}, \alpha, \beta}$ of Corollary 2.24 corresponding to $c:=c_{n}$ and satisfying (2.62). We can then define the functions $\tilde{f}_{n}:=P_{n} \circ f_{n}$ taking values in $[\alpha, \beta]$ and $h_{n}:=-c_{n} \vee f \wedge c_{n}$ taking values in $[\alpha, \beta] \cap\left[-c_{n}, c_{n}\right]$; since $\left|P_{n}(r)-P_{n}(s)\right| \leq|r-s|$ for every $r, s \in\left[-c_{n}, c_{n}\right]$ we have as $n \rightarrow \infty$

$$
\begin{aligned}
\left\|\tilde{f}_{n}-f\right\|_{L^{p}} & \leq\left\|P_{n} \circ f_{n}-P_{n} \circ h_{n}\right\|_{L^{p}}+\left\|P_{n} \circ h_{n}-h_{n}\right\|_{L^{p}}+\left\|h_{n}-f\right\|_{L^{p}} \\
& \leq\left\|f_{n}-h_{n}\right\|_{L^{p}}+\mathfrak{m}(X)^{1 / p} \varepsilon_{n}+\left\|h_{n}-f\right\|_{L^{p}} \\
& \leq\left\|f_{n}-f\right\|_{L^{p}}+\mathfrak{m}(X)^{1 / p} \varepsilon_{n}+2\left\|h_{n}-f\right\|_{L^{p}} \rightarrow 0 \quad \text { as } n \uparrow \infty .
\end{aligned}
$$

On the other hand (2.51d) yields $\operatorname{lip} \tilde{f}_{n}=\left|P_{n}^{\prime} \circ f\right| \operatorname{lip} f_{n} \leq \operatorname{lip} f_{n}$ so that

$$
\limsup _{n \rightarrow \infty} \int_{X}\left|\operatorname{lip} \tilde{f}_{n}\right|^{p} \mathrm{~d} \mathfrak{m} \leq \limsup _{n \rightarrow \infty} \int_{X}\left|\operatorname{lip} f_{n}\right|^{p} \mathrm{~d} \mathfrak{m}=\int_{X}|\mathrm{D} f|_{\star, \mathscr{A}}^{p} \mathrm{~d} \mathfrak{m} .
$$

Since $|\mathrm{D} f|_{\star, \mathscr{A}}$ is the minimal $(p, \mathscr{A})$-relaxed gradient, we also have

$$
\liminf _{n \rightarrow \infty} \int_{X}\left|\operatorname{lip} \tilde{f}_{n}\right|^{p} \mathrm{~d} \mathfrak{m} \geq \int_{X}|\mathrm{D} f|_{\star, \mathscr{A}}^{p} \mathrm{~d} \mathfrak{m},
$$

so that the sequence $\tilde{f}_{n}$ satisfies the properties stated by the Lemma.

Corollary 5.10 (Lebnitz rule). For every $f, g \in H^{1, p}(\mathbb{X}, \mathscr{A}) \cap L^{\infty}(X, \mathfrak{m})$ we have $f g \in H^{1, p}(\mathbb{X}, \mathscr{A})$ and

$$
|\mathrm{D}(f g)|_{\star, \mathscr{A}} \leq|f||\mathrm{D} g|_{\star, \mathscr{A}}+|g||\mathrm{D} f|_{\star, \mathscr{A}} .
$$

Proof. It is sufficient to approximate $f, g$ by two uniformly bounded sequences $f_{n}, g_{n} \in \mathscr{A}$ thanks to Corollary 5.9 and then pass to the limit in $(2.51 \mathrm{~b})$.

Let us now consider the locality property of the minimal $p$-relaxed gradient, by adapting the proof of [9] to the case of an arbitrary algebra $\mathscr{A}$.

Lemma 5.11 (Locality). Let $G_{1}, G_{2}$ be $(p, \mathscr{A})$-relaxed gradients of $f$. Then $\min \left\{G_{1}, G_{2}\right\}$ and $\chi_{B} G_{1}+\chi_{X \backslash B} G_{2}, B \in \mathscr{B}(X)$, are relaxed gradients of $f$ as well. In particular, for any $(p, \mathscr{A})$ relaxed gradient $G$ of $f$ it holds

$$
|\mathrm{D} f|_{\star, \mathscr{A}} \leq G \quad \mathfrak{m} \text {-a.e. in } X .
$$

Proof. It is sufficient to prove that if $B_{i} \in \mathscr{B}(X)$ with $B_{1} \cap B_{2}=\emptyset$ and $B_{1} \cup B_{2}=X$ then $\chi_{B_{1}} G_{1}+\chi_{B_{2}} G_{2}$ is a relaxed gradient of $f$. If $A \in \mathscr{B}(X)$ given by Definition 2.17, we can replace $B_{2}$ with $\tilde{B}_{2}:=B_{2} \cap A$ (and $B_{1}$ by $\tilde{B}_{1}:=X \backslash B_{2}$ ) and assume that $B_{2} \subset A$; moreover, by approximation, 
taking into account the closure of the class of relaxed gradients and the inner regularity of $\mathfrak{m}$, we can assume with no loss of generality that $B_{2}$ is a compact set (and, in particular, $B_{1}$ is open). We can also approximate $B_{1}$ by an increasing sequence of compact sets $B_{n, 1} \subset\left(B_{1} \cap A\right)$ such that $\mathfrak{m}\left(B_{1} \backslash B_{n, 1}\right) \rightarrow 0$.

Let us fix an integer $n$ and consider the compact set $K_{n}:=B_{n, 1} \cup B_{2} \subset A$; since $\mathscr{A}$ contains the constants and separates the points of $K_{n}$, the restriction of $\mathscr{A}$ to $K_{n}$ is uniformly dense in $\mathrm{C}\left(K_{n}\right)$ by Stone-Weierstrass Theorem. Being $B_{n, 1}$ and $B_{2}$ compact and disjoint, the function

$$
\chi_{n}(x):= \begin{cases}1 & \text { if } x \in B_{n, 1} \\ 0 & \text { if } x \in B_{2}\end{cases}
$$

belongs to $\mathrm{C}\left(K_{n}\right)$ so that for every $\varepsilon>0$ we can find $\tilde{\chi}_{n, \varepsilon} \in \mathscr{A}$ such that $\sup _{K_{n, 1}}\left|\tilde{\chi}_{n, \varepsilon}-\chi_{n}\right| \leq \varepsilon / 2$. If we compose $\tilde{\chi}_{n, \varepsilon}$ with the truncation polynomial $P=P_{\varepsilon / 2}^{c, 0,1}$ of Corollary 2.24 corresponding $c:=1+\sup \left|\chi_{n, \varepsilon}\right|$, we obtain the function $\chi_{n, \varepsilon}:=P \circ \tilde{\chi}_{n, \varepsilon}$ taking values in $[0,1]$ and satisfying

$$
\sup _{K_{n}}\left|\chi_{n, \varepsilon}-P \circ \chi_{n}\right| \leq \varepsilon / 2, \quad \sup _{K_{n}}\left|P \circ \chi_{n}-\chi_{n}\right| \leq \varepsilon / 2
$$

since $0 \vee \chi_{n} \wedge 1=\chi_{n}$ on $K_{n}$. We deduce that

$$
0 \leq \chi_{n, \varepsilon} \leq 1, \quad 0 \leq \chi_{n, \varepsilon} \leq \varepsilon \text { on } B_{2}, \quad 1-\varepsilon \leq \chi_{n, \varepsilon} \leq 1 \text { on } B_{n, 1} .
$$

Let now $h_{k, i} \in \mathscr{A}, i=1,2$, functions converging to $f$ in $L^{p}$ as $k \rightarrow \infty$ with lip $h_{k, i}$ weakly converging to $\tilde{G}_{i} \leq G_{i}$, and set $f_{k, n, \varepsilon}:=\chi_{n, \varepsilon} h_{k, 1}+\left(1-\chi_{n, \varepsilon}\right) h_{k, 2} \in \mathscr{A}$. Passing first to the limit as $k \uparrow+\infty$, since $f_{k, n, \varepsilon} \rightarrow f,(2.51 \mathrm{c})$ immediately gives that $G_{n, \varepsilon}:=\chi_{n, \varepsilon} G_{1}+\left(1-\chi_{n, \varepsilon}\right) G_{2} \geq$ $\chi_{n, \varepsilon} \tilde{G}_{1}+\left(1-\chi_{n, \varepsilon}\right) \tilde{G}_{2}$ is a relaxed gradient of $f$.

We can now select a vanishing sequence $\left(\varepsilon_{j}\right)_{j \in \mathbb{N}}$ and we pass to the limit as $j \uparrow+\infty$, obtaining (possibly extracting a further subsequence) a limit function $\chi_{n}$ taking values in $[0,1]$ such that $G_{n}:=$ $\chi_{n} G_{1}+\left(1-\chi_{n}\right) G_{2} \geq \chi_{n} \tilde{G}_{1}+\left(1-\chi_{n}\right) \tilde{G}_{2}$ is a relaxed gradient and $\chi_{\left.n\right|_{B_{2}}}=0,\left.\chi_{n}\right|_{B_{n, 1}}=1$. We can finally pass to the limit as $n \rightarrow \infty$, observing that $\chi_{n}$ converges pointwise $\mathfrak{m}$-a.e. to the characteristic function of $B$.

For the second part of the statement we argue by contradiction: let $G$ be a relaxed gradient of $f$ and assume that there exists a Borel set $B$ with $\mathfrak{m}(B)>0$ on which $G<|\mathrm{D} f|_{\star, \mathscr{A}}$. Consider the relaxed gradient $G \chi_{B}+|\mathrm{D} f|_{\star, \mathscr{A}} \chi_{X \backslash B}$ : its $L^{p}$ norm is strictly less than the $L^{p}$ norm of $|\mathrm{D} f|_{\star, \mathscr{A}}$, which is a contradiction.

Theorem 5.12. For every $f, g \in H^{1, p}(\mathbb{X}, \mathscr{A})$ we have

(a) (Pointwise sublinearity) For $|\mathrm{D}(\alpha f+\beta g)|_{\star, \mathscr{A}} \leq \alpha|\mathrm{D} f|_{\star, \mathscr{A}}+\beta|\mathrm{D} g|_{\star, \mathscr{A}}$.

(b) (Locality) For any Borel set $N \subset \mathbb{R}$ with $\mathscr{L}^{1}(N)=0$ we have

$$
|\mathrm{D} f|_{\star, \mathscr{A}}=0 \quad \mathfrak{m} \text {-a.e. on } f^{-1}(N) .
$$

In particular for every constant $c \in \mathbb{R}$

$$
|\mathrm{D} f|_{\star, \mathscr{A}}=|\mathrm{D} g|_{\star, \mathscr{A}} \mathfrak{m} \text {-a.e. on }\{f-g=c\} .
$$

(c) (Chain rule) If $\phi \in \operatorname{Lip}(\mathbb{R})$ then $\phi \circ f \in H^{1, p}(\mathbb{X}, \mathscr{A})$ with

$$
|\mathrm{D}(\phi \circ f)|_{\star, \mathscr{A}} \leq\left|\phi^{\prime}(f)\right||\mathrm{D} f|_{\star, \mathscr{A}} .
$$

Equality holds in (5.16) if $\phi$ is monotone or $\mathrm{C}^{1}$. 
(d) (Normal contractions) If $\phi: \mathbb{R} \rightarrow \mathbb{R}$ is a nondecreasing contraction and $\tilde{f}=f+\phi(g-f)$, $\tilde{g}=g+\phi(f-g)$ then

$$
|\mathrm{D} \tilde{f}|_{\star, \mathscr{A}}^{p}+|\mathrm{D} \tilde{g}|_{\star, \mathscr{A}}^{p} \leq|\mathrm{D} f|_{\star, \mathscr{A}}^{p}+|\mathrm{D} g|_{\star, \mathscr{A}}^{p}
$$

Proof. (a) follows immediately by the convexity of the set $S$ defined by (5.8) and (5.12).

(b) We first claim that for $\phi: \mathbb{R} \rightarrow \mathbb{R}$ continuously differentiable whose derivative $\phi^{\prime}$ is Lipschitz on the image of $f$ it holds

$$
|\mathrm{D} \phi(f)|_{\star, \mathscr{A}} \leq\left|\phi^{\prime} \circ f \| \mathrm{D} f\right|_{\star, \mathscr{A}}, \quad \mathfrak{m} \text {-a.e. in } X
$$

for any $f \in H^{1, p}(\mathbb{X}, \mathscr{A})$. (5.18) easily follows by approximation from (2.51d) whenever $f$ is bounded and $\phi$ is a polynomial: it is sufficient to apply Corollary 5.9.

Still assuming the boundedness of $f$, arbitrary $\mathrm{C}^{1}$ functions $\phi$ can be approximated by a sequence of polynomials $P_{n}$ with respect to the $\mathrm{C}^{1}$-norm induced by a compact interval containing $f(X)$. Thanks to the weak closure of (5.8) we can pass to the limit in (5.18) written for $P_{n}$ and obtain the same bound for $\phi$. In particular, for every $f \in \mathscr{A}$ we get

$$
|\mathrm{D}(\phi \circ f)|_{\star, \mathscr{A}} \leq\left|\phi^{\prime} \circ f\right||\mathrm{D} f|_{\star, \mathscr{A}} \leq\left|\phi^{\prime} \circ f\right| \operatorname{lip} f \quad \mathfrak{m} \text {-a.e. in } X .
$$

If now $\phi \in \mathrm{C}^{1}(\mathbb{R}) \cap \operatorname{Lip}(\mathbb{R})$ and $f \in H^{1, p}(\mathbb{X}, \mathscr{A})$ we can use the approximation (5.9) and (5.19) to obtain a sequence $f_{n} \in \mathscr{A}$ such that

$$
\begin{aligned}
\phi \circ f_{n} & \rightarrow \phi \circ f & & \text { in } L^{p}(X, \mathfrak{m}), \\
\left|\mathrm{D}\left(\phi \circ f_{n}\right)\right|_{\star, \mathscr{A}} & \rightarrow G & & \text { in } L^{p}(X, \mathfrak{m}), \\
\left|\phi^{\prime} \circ f_{n}\right|\left|\mathrm{D} f_{n}\right|_{\star, \mathscr{A}} & \rightarrow\left|\phi^{\prime} \circ f\right||\mathrm{D} f|_{\star, \mathscr{A}} & & \text { in } L^{p}(X, \mathfrak{m}),
\end{aligned}
$$

so that $|\mathrm{D}(\phi \circ f)|_{\star, \mathscr{A}} \leq G \leq\left|\phi^{\prime} \circ f\right||\mathrm{D} f|_{\star, \mathscr{A}}$.

Now, assume that $N$ is compact. In this case, let $A_{n} \subset \mathbb{R}$ be open sets such that $A_{n} \downarrow N$ and $\mathscr{L}^{1}\left(A_{1}\right)<\infty$. Also, let $\psi_{n}: \mathbb{R} \rightarrow[0,1]$ be a continuous function satisfying $\chi_{N} \leq \psi_{n} \leq \chi_{A_{n}}$, and define $\phi_{n}: \mathbb{R} \rightarrow \mathbb{R}$ by

$$
\left\{\begin{array}{l}
\phi_{n}(0)=0 \\
\phi_{n}^{\prime}(z)=1-\psi_{n}(z)
\end{array}\right.
$$

The sequence $\left(\phi_{n}\right)$ uniformly converges to the identity map, and each $\phi_{n}$ is 1-Lipschitz and $C^{1}$. Therefore $\phi_{n} \circ f$ converge to $f$ in $L^{2}$. Taking into account that $\phi_{n}^{\prime}=0$ on $N$ and (5.18) we deduce

$$
\begin{aligned}
\int_{X}|\mathrm{D} f|_{\star, \mathscr{A}}^{p} \mathrm{~d} \mathfrak{m} & \leq \liminf _{n \rightarrow \infty} \int_{X}\left|\mathrm{D} \phi_{n}(f)\right|_{\star, \mathscr{A}}^{p} \mathrm{~d} \mathfrak{m} \leq \liminf _{n \rightarrow \infty} \int_{X}\left|\phi_{n}^{\prime} \circ f\right|^{p}|\mathrm{D} f|_{\star, \mathscr{A}}^{p} \mathrm{dm} \\
& =\liminf _{n \rightarrow \infty} \int_{X \backslash f^{-1}(N)}\left|\phi_{n}^{\prime} \circ f\right|^{p}|\mathrm{D} f|_{\star, \mathscr{A}}^{p} \mathrm{~d} \mathfrak{m} \leq \int_{X \backslash f^{-1}(N)}|\mathrm{D} f|_{\star, \mathscr{A}}^{p} \mathrm{dm} .
\end{aligned}
$$

It remains to deal with the case when $N$ is not compact. In this case we consider the finite measure $\mu:=f_{\sharp} \mathfrak{m}$. Then there exists an increasing sequence $\left(K_{n}\right)$ of compact subsets of $N$ such that $\mu\left(K_{n}\right) \uparrow$ $\mu(N)$. By the result for the compact case we know that $|\mathrm{D} f|_{\star, \mathscr{A}}=0 \mathfrak{m}$-a.e. on $\cup_{n} f^{-1}\left(K_{n}\right)$, and by definition of push forward we know that $\mathfrak{m}\left(f^{-1}\left(N \backslash \cup_{n} K_{n}\right)\right)=0$.

(5.15) then follows if $g$ is identically 0 . In the general case we notice that $|\mathrm{D}(f-g)|_{\star, \mathscr{A}}+|\mathrm{D} g|_{\star, \mathscr{A}}$ is a relaxed gradient of $f$, hence on $\{f-g=c\}$ we conclude that $\mathfrak{m}$-a.e. it holds $|\mathrm{D} f|_{\star, \mathscr{A}} \leq|\mathrm{D} g|_{\star, \mathscr{A}}$. 
Reversing the roles of $f$ and $g$ we conclude.

(c) By 2. and Rademacher Theorem we know that the right hand side is well defined, so that the statement makes sense (with the convention to define $\left|\phi^{\prime} \circ f\right|$ arbitrarily at points $x$ such that $\phi^{\prime}$ does not exist at $f(x)$ ). Also, by (5.18) we know that the thesis is true if $\phi$ is $C^{1}$. For the general case, just approximate $\phi$ with a sequence $\left(\phi_{n}\right)$ of equi-Lipschitz and $C^{1}$ functions, such that $\phi_{n}^{\prime} \rightarrow \phi^{\prime}$ a.e. on the image of $f$.

Let us now consider the monotone case; with no loss of generality we can assume that $0 \leq \phi^{\prime} \leq$ 1. We know that $\left(1-\phi^{\prime}(f)\right)|\mathrm{D} f|_{\star, \mathscr{A}}$ and $\phi^{\prime}(f)|\mathrm{D} f|_{\star, \mathscr{A}}$ are relaxed gradients of $f-\phi(f)$ and $f$ respectively. Since

$$
|\mathrm{D} f|_{\star, \mathscr{A}} \leq|\mathrm{D}(f-\phi(f))|_{\star, \mathscr{A}}+|\mathrm{D} \phi(f)|_{\star, \mathscr{A}} \leq\left(\left(1-\phi^{\prime}(f)\right)+\phi^{\prime}(f)\right)|\mathrm{D} f|_{\star, \mathscr{A}}=|\mathrm{D} f|_{\star, \mathscr{A}}
$$

it follows that all inequalities are equalities $\mathfrak{m}$-a.e. in $X$.

When $\phi$ is $\mathrm{C}^{1}$ we can use the locality property.

(d) Applying Lemma 5.6 we find two optimal sequences $\left(f_{n}\right),\left(g_{n}\right)$ of bounded Lipschitz functions satisfying (5.9) (w.r.t. $f$ and $g$ respectively). When $\phi$ is of class $C^{1}$, passing to the limit in the inequality (2.51e) written for $f_{n}$ and $g_{n}$ we easily get (5.17). In the general case, we first approximate $\phi$ by a sequence $\phi_{n}$ of nondecreasing contraction of class $C^{1}$ converging to $\phi$ pointwise and then pass to the limit in (5.17) written for $\phi_{n}$.

Corollary 5.13. If $f_{1}, \cdots, f_{M} \in H^{1, p}(\mathbb{X}, \mathscr{A})$ then also the functions $f_{+}:=f_{1} \vee f_{2} \vee \cdots \vee f_{M}$ and $f_{-}:=f_{1} \wedge f_{2} \wedge \cdots \wedge f_{M}$ belong to $H^{1, p}(\mathbb{X}, \mathscr{A})$ and

$$
\begin{array}{ll}
\left|\mathrm{D} f_{+}\right|_{\star, \mathscr{A}}=\left|\mathrm{D} f_{j}\right|_{\star, \mathscr{A}} & \text { on } A_{j}:=\left\{x \in X: f_{+}=f_{j}\right\}, \\
\left|\mathrm{D} f_{-}\right|_{\star, \mathscr{A}}=\left|\mathrm{D} f_{j}\right|_{\star, \mathscr{A}} & \text { on } B_{j}:=\left\{x \in X: f_{-}=f_{j}\right\} .
\end{array}
$$

\subsection{Invariance w.r.t. restriction and completion}

It is obvious that the Cheeger energy and the minimal relaxed gradient are invariant with respect to isomorphisms of e.m.t.m. structures $(\mathbb{X}, \mathscr{A})$, according to Definition 2.28. Here we state two simple (and very preliminary) results concerning the behaviour of the Cheeger energy w.r.t. a general measure-preserving embedding $\iota$ of $(\mathbb{X}, \mathscr{A})$ into $\left(\mathbb{X}^{\prime}, \mathscr{A}^{\prime}\right)$ : we keep the same notation of Section 2.7 . We will state a much deeper result in the last Section of these notes, see Theorem 12.3.

Lemma 5.14. For every $f^{\prime} \in H^{1, p}\left(\mathbb{X}^{\prime}, \mathscr{A}^{\prime}\right)$ the function $f:=\iota^{*} f^{\prime}$ belongs to $H^{1, p}(\mathbb{X}, \mathscr{A})$ and

$$
|\mathrm{D} f|_{\star, \mathscr{A}} \leq \iota^{*}\left(\left|\mathrm{D} f^{\prime}\right|_{\star, \mathscr{A}^{\prime}}\right) \quad \mathfrak{m} \text {-a.e. in } X .
$$

Proof. Let us first observe that if $f^{\prime} \in \operatorname{Lip}_{b}\left(X^{\prime}, \tau^{\prime}, \mathrm{d}^{\prime}\right)$ and $G^{\prime} \geq \operatorname{lip}_{\mathrm{d}^{\prime}} f^{\prime}$, then

$$
\iota^{*} G^{\prime} \geq \operatorname{lip}_{\mathrm{d}}\left(\iota^{*} f^{\prime}\right)
$$

In fact, setting $f:=\iota^{*}\left(f^{\prime}\right)$ and choosing arbitrary sets $U \in X$ and $U^{\prime} \in X^{\prime}$ containing $\iota(U)$, if $L=\operatorname{Lip}\left(f^{\prime}, U^{\prime}, \mathrm{d}^{\prime}\right)$ we have

$$
|f(x)-f(y)|=\left|f^{\prime}(\iota(x))-f^{\prime}(\iota(y))\right| \leq L \mathrm{~d}^{\prime}(\iota(x), \iota(y))=L \mathrm{~d}(x, y) \quad \text { for every } x, y \in U
$$

so that

$$
\operatorname{Lip}(f, U, \mathrm{~d}) \leq \operatorname{Lip}\left(f^{\prime}, U^{\prime}, \mathrm{d}^{\prime}\right)
$$


Recalling the definition (2.48) and considering the collection of all the open neighborhood of $\iota(x)$ in $X^{\prime}$, we get (5.22).

In order to obtain (5.21) it is now sufficient to take an optimal sequence $f_{n}^{\prime}, G_{n}^{\prime}$ as in (5.9) for the $\left(p, \mathscr{A}^{\prime}\right)$-minimal relaxed gradient $\left|\mathrm{D} f^{\prime}\right|_{\star, \mathscr{A}^{\prime}}$ of $f^{\prime} \in H^{1, p}\left(\mathbb{X}^{\prime}, \mathscr{A}^{\prime}\right)$ observing that

$$
\iota^{*} f_{n}^{\prime} \rightarrow f, \quad \iota^{*} G_{n}^{\prime} \rightarrow \iota^{*}\left|\mathrm{D} f^{\prime}\right|_{\star} \quad \text { strongly in } L^{p}(X, \mathfrak{m}),
$$

so that $\iota^{*}\left|\mathrm{D} f^{\prime}\right|_{\star, \mathscr{A}^{\prime}}$ is a relaxed gradient for $f$.

When $\iota(X)$ is $\mathrm{d}^{\prime}$-dense in $X^{\prime}$ (in particular, when $X^{\prime}$ is a completion according to the definition given in Corollary 2.36), we have a better behaviour.

Proposition 5.15. Suppose that $\iota: X \rightarrow X^{\prime}$ is a measure-preserving embedding of $(\mathbb{X}, \mathscr{A})$ into $\left(X^{\prime}, \mathscr{A}^{\prime}\right)$ such that

$$
\iota(X) \text { is } \mathrm{d}^{\prime} \text {-dense in } X^{\prime}, \quad \iota^{*}\left(\mathscr{A}^{\prime}\right)=\mathscr{A} .
$$

Then $\iota^{*}$ is an isomorphism of $H^{1, p}\left(\mathbb{X}^{\prime}, \mathscr{A}^{\prime}\right)$ onto $H^{1, p}(\mathbb{X}, \mathscr{A})$ and for every $f=\iota^{*} f^{\prime}$

$$
|\mathrm{D} f|_{\star, \mathscr{A}}=\iota^{*}\left(\left|\mathrm{D} f^{\prime}\right|_{\star, \mathscr{A}^{\prime}}\right) \quad \mathfrak{m} \text {-a.e. in } X .
$$

Proof. Let $f \in H^{1, p}(\mathbb{X}, \mathscr{A})$ and let $f_{n} \in \mathscr{A}$ be an optimal approximating sequence as in (5.9).

We want to show that $f^{\prime}=\iota_{*} f \in H^{1, p}\left(\mathbb{X}^{\prime}, \mathscr{A}^{\prime}\right)$; by Lemma 2.31 and (5.24), we can find $f_{n}^{\prime} \in \mathscr{A}^{\prime}$ such that $f_{n}=\iota^{*} f_{n}^{\prime}$. Since $\iota_{*}$ is an $L^{p}$-isometry, we know that $f_{n}^{\prime} \rightarrow f^{\prime}$ strongly in $L^{p}\left(X^{\prime}, \mathfrak{m}^{\prime}\right)$. Since $\iota$ is a homeomorphism between $X$ and $\iota(X)$, if $x^{\prime}=\iota(x)$ and $g>\operatorname{lip}_{\mathrm{d}} f_{n}(x)$, we can find an open neighborhood $U^{\prime}$ of $x^{\prime}$ such that setting $U:=\iota^{-1}\left(U^{\prime}\right)$ we have

$$
\left|f_{n}(z)-f_{n}(y)\right|=\left|f_{n}^{\prime}(\iota(z))-f_{n}^{\prime}(\iota(y))\right| \leq g \mathrm{~d}^{\prime}(\iota(z), \iota(y))=g \mathrm{~d}(z, y) \quad \text { for every } z, y \in U .
$$

On the other hand, the d-density of $\iota(X)$ in $X^{\prime}$ guarantees that for every $z^{\prime}, y^{\prime} \in U^{\prime} \backslash(\iota(U))$ there exist $\mathrm{d}^{\prime}$ balls $B_{\delta}\left(z^{\prime}\right), B_{\delta}\left(y^{\prime}\right)$ of radius $\delta$ such that

$$
B_{\delta}\left(z^{\prime}\right) \subset U^{\prime}, B_{\delta}\left(y^{\prime}\right) \subset U^{\prime}, \quad B_{\delta}\left(z^{\prime}\right) \cap \iota(U) \neq \emptyset, B_{\delta}\left(y^{\prime}\right) \cap \iota(U) \neq \emptyset,
$$

so that (5.26) extends to $U^{\prime}$ as

$$
\left|f_{n}^{\prime}\left(z^{\prime}\right)-f_{n}^{\prime}\left(y^{\prime}\right)\right| \leq g \mathrm{~d}\left(y^{\prime}, z^{\prime}\right) \quad \text { for every } z, y \in U .
$$

We deduce that

$$
\operatorname{lip}_{\mathrm{d}^{\prime}} f_{n}^{\prime}(\iota(x)) \leq \operatorname{lip} f_{n}(x) \quad \text { for every } x \in X
$$

and therefore

$$
\begin{aligned}
\limsup _{n \rightarrow \infty} \int_{X^{\prime \prime}}\left(\operatorname{lip}_{\mathrm{d}^{\prime}} f_{n}^{\prime}\right)^{p} \mathrm{dm}^{\prime} & =\limsup _{n \rightarrow \infty} \int_{X^{\prime \prime}}\left(\operatorname{lip}_{\mathrm{d}^{\prime}} f_{n}^{\prime}(\iota(x))^{p} \mathrm{~d} \mathfrak{m}(x)\right. \\
& \leq \lim _{n \rightarrow \infty} \int_{X}\left(\operatorname{lip} f_{n}(x)\right)^{p} \mathrm{~d} \mathfrak{m}(x)=\mathrm{CE}_{p, \mathscr{A}}(f)
\end{aligned}
$$

We obtain that

$$
f^{\prime} \in H^{1, p}\left(\mathbb{X}^{\prime}, \mathscr{A}^{\prime}\right), \quad \mathrm{CE}_{p, \mathscr{A}^{\prime \prime}}\left(f^{\prime}\right) \leq \mathrm{CE}_{p, \mathscr{A}}(f) .
$$

Thanks to (5.21) we also get (5.25). 
As an immediate application we obtain that the class of complete e.m.t.m. spaces is the natural setting for the Cheeger energy.

Corollary 5.16 (Invariance of the Cheeger energy by completion). If $\overline{\mathbb{X}}=(\bar{X}, \bar{\tau}, \overline{\mathrm{d}}, \overline{\mathfrak{m}})$ is the completion of $\mathbb{X}$ induced by $\iota: X \rightarrow \bar{X}$ of Corollary 2.36 and $\overline{\mathscr{A}}=\{\bar{f}: f \in \mathscr{A}\}$, then $\iota^{*}$ is an isomorphism of $H^{1, p}(\overline{\mathbb{X}}, \bar{A})$ onto $H^{1, p}(\mathbb{X}, \mathscr{A})$ and

$$
|\mathrm{D} f|_{\star, \mathscr{A}}=\iota^{*}\left(|\mathrm{D} \bar{f}|_{\star, \bar{A}}\right) \quad \mathfrak{m} \text {-a.e. in } X \quad \text { for every } f=\iota^{*} \bar{f} .
$$

We conclude with another easy application of the previous results to restrictions, as in Example 2.30(c). Recall that if $Y \subset X$ is a $\tau$-dense $\mathfrak{m}$-measurable subset satisfying $\mathfrak{m}(X \backslash Y)=0$, the restriction to $Y$ is an isomorphism of $L^{p}(X, \mathfrak{m})$ with $L^{p}\left(Y, \mathfrak{m}_{Y}\right)$, so that one can compare the Sobolev spaces $H^{1, p}(\mathbb{X}, \mathscr{A})$ and $H^{1, p}\left(\mathbb{Y}, \mathscr{A}_{Y}\right)$. We will denote by $|\mathrm{D} f|_{\star, \mathbb{Y}, \mathscr{A}_{Y}}$ the $\left(p, \mathscr{A}_{Y}\right)$ minimal relaxed gradient in $H^{1, p}\left(\mathbb{Y}, \mathscr{A}_{Y}\right)$.

Corollary 5.17 (Restriction). Let $\mathbb{X}=(X, \tau, \mathrm{d}, \mathfrak{m})$ be an e.m.t.m. space and let $Y \subset X$ be a $\tau$-dense $\mathfrak{m}$-measurable subset satisfying $\mathfrak{m}(X \backslash Y)=0$. With the above notation, (the restriction to $Y$ of) every function $f \in H^{1, p}(\mathbb{X}, \mathscr{A})$ belongs to $H^{1, p}\left(\mathbb{Y}, \mathscr{A}_{Y}\right)$ and

$$
|\mathrm{D} f|_{\star, \mathbb{Y}, \mathscr{A}_{Y}} \leq|\mathrm{D} f|_{\star, \mathscr{A}} \quad \mathfrak{m} \text {-a.e. }
$$

If moreover $Y$ is $\mathrm{d}$-dense in $X$, then the converse property is also true: for every $f \in L^{p}(X, \mathfrak{m})$

$$
f \in H^{1, p}(\mathbb{X}, \mathscr{A}) \quad \Leftrightarrow \quad H^{1, p}\left(\mathbb{Y}, \mathscr{A}_{Y}\right), \quad|\mathrm{D} f|_{\star, \mathbb{Y}, \mathscr{A}_{Y}}=|\mathrm{D} f|_{\star, \mathscr{A}} \quad \mathfrak{m} \text {-a.e. }
$$

\subsection{Notes}

$\S 5.1$ is strongly inspired by Cheeger's work [23] (where the energy is obtained starting from upper gradients instead of the local Lipschitz constants) and follows quite closely the presentation of $[9,8]$, with the required adjustments due to the presence of a compatible algebra $\mathscr{A}$ instead of $\operatorname{Lip}_{b}(X, \tau, \mathrm{d})$. Corollary 5.9 and the crucial locality Lemma 5.11 take advantage of the approximation tools presented in $\S 2.6$. Even if a posteriori the Cheeger energy will be independent of $\mathscr{A}$, the role of the algebra should be considered as a technique to get new density results. Moreover, it allows for simpler constructions in many cases, where a distinguished algebra provides better structural properties of the energy, see the final Section 12.

$\S 5.2$ contains some preliminary facts about the behaviour of the Cheeger energy with respect to measurepreserving embeddings of e.m.t.m. spaces (in particular w.r.t. completion). The possibility to modify the topological and the algebraic properties of the e.m.t.m. setting is one of its strength point.

\section{Invariance of the Cheeger energy with respect to the core algebra: the compact case}

The aim of this section is to study the property of the Cheeger energy with respect to the choice of the core algebra $\mathscr{A}$ in the case of a compact ambient space $(X, \tau)$. An important tool is provided by the (generalized) Hopf-Lax flow, which we collect in the next section. 


\subsection{The metric Hopf-Lax flow in compact spaces}

Let $(X, \tau, \mathrm{d})$ be a compact extended metric-topological space and let $\delta: X \times X \rightarrow[0,+\infty]$ be a $\tau$-l.s.c. continuous extended semidistance (our main examples will be the extended distance $\mathrm{d}$ and the continuous semidistances $\mathrm{d}_{i}$ as in Lemma 2.4). For every $f \in \mathrm{C}_{b}(X), x, y \in X$ and $t>0$ we set

$$
F^{\delta}(t, x, y):=f(y)+\frac{\delta^{q}(x, y)}{q t^{q-1}}, \quad F(t, x, y):=F^{\mathrm{d}}(t, x, y) .
$$

$F^{\delta}$ is a l.s.c. (continuous, if $\delta$ is continuous) function bounded from below.

Let us also fix a compact set $K \subset X$ such that there exists a constant $S=S(K, \delta) \in[0,+\infty[$ satisfying

$$
\min _{y \in K} \delta(x, y) \leq S \quad \text { for every } x \in X .
$$

(6.2) is always satisfied if $\delta$ is continuous or if $K=X$ (and in this case $S=0$ ).

The modified Hopf-Lax evolution is defined by the formula

$$
\mathrm{Q}_{t}^{K, \delta} f(x):=\min _{y \in K} F^{\delta}(t, x, y) \quad t>0,
$$

where we will omit to indicate the explicit dependence on $K$ (resp. on $\delta$ ) when $K=X$ (resp. when $\delta=\mathrm{d}$ ), thus setting

$$
\mathrm{Q}_{t}^{\delta} f:=\mathrm{Q}_{t}^{X, \delta} f, \quad \mathrm{Q}_{t} f:=\mathrm{Q}_{t}^{X, \mathrm{~d}} f .
$$

Since $K$ is compact and $F^{\delta}(t, x, \cdot)$ takes at least one finite value in $K$ by (6.2), the minimum in (6.3) is attained: for every $x \in X$ we also set

$$
\mathrm{J}_{t}^{K, \delta} f(x):=\left\{y \in K: f(y)+\frac{\delta^{q}(x, y)}{p t^{q-1}}=\mathrm{Q}_{t}^{\delta} f(x)\right\}, \quad \mathrm{J}_{t}^{\delta}:=\mathrm{J}_{t}^{X, \delta}, \quad \mathrm{J}_{t}:=\mathrm{J}_{t}^{X, \mathrm{~d}}
$$

and

$$
\mathrm{D}_{t}^{K, \delta,+} f(x):=\max _{y \in \mathrm{J}_{t}^{K, \delta} f(x)} \delta(x, y), \quad \mathrm{D}_{t}^{K, \delta,-} f(x):=\min _{y \in \mathrm{J}_{t}^{K, \delta} f(x)} \delta(x, y) .
$$

As usual, we set $\operatorname{Osc}(f, X):=\sup _{X} f-\inf _{X} f$.

Lemma 6.1 (Basic estimates). Let $f \in \mathrm{C}_{b}(X)$ and let $f_{t}(x):=\mathrm{Q}_{t}^{K, \delta} f(x), J_{t}(x):=\mathrm{J}_{t}^{K, \delta} f$, $D_{t}^{ \pm}(x):=\mathrm{D}_{t}^{K, \delta, \pm} f(x)$ be defined as (6.3), (6.5), (6.6) for $t>0$. For every $x, y \in X, 0<s<t$, $x^{\prime} \in J_{t}(x), y^{\prime} \in J_{s}(y)$ we have

$$
\begin{gathered}
\min _{X} f \leq f_{t}(x) \leq \max _{X} f+\frac{1}{q t^{q-1}} S^{q} \quad \text { for every } t>0, x \in X, \\
\left(\frac{D_{t}^{+}(x)}{t}\right)^{q} \leq \min \left(q t^{-1} \operatorname{Osc}(f, X),(q \operatorname{Lip}(f, X, \delta))^{p}\right), \\
\left(\frac{1}{q s^{q-1}}-\frac{1}{q t^{q-1}}\right) D_{s}^{+}(x) \leq f_{s}(x)-f_{t}(x) \leq\left(\frac{1}{q s^{q-1}}-\frac{1}{q t^{q-1}}\right) D_{t}^{-}(x), \\
-\left(\frac{\delta\left(x, y^{\prime}\right)}{t}\right)^{q-1} \delta(x, y)+\frac{1}{p}\left(\frac{\delta\left(y, y^{\prime}\right)}{t}\right)^{q}(t-s) \leq f_{s}(y)-f_{t}(x) \\
\leq\left(\frac{\delta\left(y, x^{\prime}\right)}{s}\right)^{q-1} \delta(x, y)+\frac{1}{p}\left(\frac{\delta\left(x, x^{\prime}\right)}{s}\right)^{q}(t-s) .
\end{gathered}
$$


Proof. (6.7) is immediate. In order to prove (6.8) we simply observe that for every $x^{\prime} \in J_{t}(x)$

$$
\frac{\delta^{q}\left(x, x^{\prime}\right)}{t^{q-1}} \leq q\left(f(x)-f\left(x^{\prime}\right)\right) \leq q \min \left(\operatorname{Osc}(f, X), \operatorname{Lip}(f, X, \delta) \delta\left(x, x^{\prime}\right)\right)
$$

thus obtaining

$$
\left(\frac{\delta\left(x, x^{\prime}\right)}{t}\right)^{q} \leq \frac{q}{t} \operatorname{Osc}(f, X), \quad\left(\frac{\delta\left(x, x^{\prime}\right)}{t}\right)^{q-1} \leq q \operatorname{Lip}(f, X, \delta) .
$$

Let us now check (6.11): selecting $x^{\prime} \in J_{t}(x)$

$$
\begin{aligned}
f_{s}(y)-f_{t}(x) & \leq F^{\delta}\left(s, y, x^{\prime}\right)-F^{\delta}\left(t, x, x^{\prime}\right)=\frac{\delta^{q}\left(y, x^{\prime}\right)}{q s^{q-1}}-\frac{\delta^{q}\left(x, x^{\prime}\right)}{q t^{q-1}} \\
& =\frac{\delta^{q}\left(y, x^{\prime}\right)}{q s^{q-1}}-\frac{\delta^{q}\left(x, x^{\prime}\right)}{q s^{q-1}}+\left(\frac{1}{q s^{q-1}}-\frac{1}{q t^{q-1}}\right) \delta^{q}\left(x, x^{\prime}\right) \\
& \leq\left(\frac{\delta\left(y, x^{\prime}\right)}{s}\right)^{q-1}\left|\delta\left(y, x^{\prime}\right)-\delta\left(x, x^{\prime}\right)\right|+\left(\frac{1}{q s^{q-1}}-\frac{1}{q t^{q-1}}\right) \delta^{q}\left(x, x^{\prime}\right) .
\end{aligned}
$$

Applying the triangle inequality for $\delta$ and the elementary inequality (arising from the convexity of $r \mapsto 1 / r^{q-1}$ in $\left.(0, \infty)\right)$

$$
\frac{1}{p t^{q}}(t-s) \leq \frac{1}{q s^{q-1}}-\frac{1}{q t^{q-1}} \leq \frac{1}{p s^{q}}(t-s) \text { for every } s, t \in(0,+\infty)
$$

we obtain (6.11). (6.10) will follow by switching the role of $(x, t)$ and $(y, s)$.

Concerning (6.9), the right inequality can be easily obtained by choosing $y=x$ in (6.12) and minimizing with respect to $x^{\prime} \in J_{t}(x)$. Inverting the role of $s$ and $t$ (notice that (6.12) does not require $s<t$ ) and maximizing with respect to $x^{\prime} \in J_{s}(x)$ we get the left inequality of (6.9).

We collect further properties in the next Lemma.

Lemma 6.2. Let us assume that $\delta$ is continuous. For every $f \in \mathrm{C}(X)$ we have

(a) The map $(x, t) \mapsto Q_{t}^{K, \delta} f(x)$ is continuous in $X \times(0,+\infty)$ and for every $0<s<t$ it satisfies the estimate

$$
\frac{1}{p}\left(\frac{\mathrm{D}_{s}^{K, \delta,+} f(x)}{t}\right)^{q}(t-s) \leq \mathrm{Q}_{s}^{K, \delta} f(x)-\mathrm{Q}_{t}^{K, \delta} f(x) \leq \frac{1}{p}\left(\frac{\mathrm{D}_{t}^{K, \delta,-} f(x)}{s}\right)^{q}(t-s) .
$$

(b) The map $(x, t) \mapsto \mathrm{D}_{t}^{K, \delta,+} f(x)$ (resp. $\left.(x, t) \mapsto \mathrm{D}_{t}^{K, \delta,-} f(x)\right)$ is upper (resp. lower) semicontinuous in $X \times(0, \infty)$ and there holds

$$
\mathrm{D}_{s}^{K, \delta,-} f(x) \leq \mathrm{D}_{s}^{K, \delta,+} f(x) \leq \mathrm{D}_{t}^{K, \delta,-} f(x) \quad \text { if } 0<s<t .
$$

(c) If $\left(K_{\lambda}\right)_{\lambda \in \Lambda}$ is an increasing net with $\cup_{\lambda \in \Lambda} K_{\lambda}$ dense in $K$, then for every $x \in X$ the net $\lambda \mapsto \mathrm{Q}_{t}^{K_{\lambda}, \delta} f(x)$ is decreasing and converging to $\mathrm{Q}_{t}^{K, \delta} f(x)$.

(d) If $\left(K_{\lambda}\right)_{\lambda \in \Lambda}$ is an increasing net with $K_{\lambda} \subset K$, then

$$
\lim _{\lambda \in \Lambda} \mathrm{Q}_{t}^{K_{\lambda}, \delta} f(x)=\mathrm{Q}_{t}^{K, \delta} f(x) \Rightarrow \limsup _{\lambda \in \Lambda} \mathrm{D}_{t}^{K_{\lambda}, \delta,+} f(x) \leq \mathrm{D}_{t}^{K, \delta,+} f(x) .
$$


Proof. (a) Continuity follows by (6.10) and (6.11). (6.14) is a consequence of (6.9) and (6.13).

(b) (6.15) follows immediately from (6.9). It is not difficult to check that $(t, x) \mapsto \mathrm{D}_{t}^{K, \delta,+} f(x)$ is upper semicontinuous: if $\left(x_{\lambda}, t_{\lambda}\right), \lambda \in \Lambda$, is a net converging to $(x, t) \in X \times(0,+\infty)$ with $\mathrm{D}_{t_{\lambda}}^{K, \delta,+}\left(x_{\lambda}\right) \geq c$ and $y_{\lambda} \in \mathrm{J}_{t_{\lambda}}^{K, \delta}\left(x_{\lambda}\right)$ such that

$$
F^{\delta}\left(t_{\lambda}, x_{\lambda}, y_{\lambda}\right)=\mathrm{Q}_{t_{\lambda}}^{K, \delta} f\left(x_{\lambda}\right), \quad \delta\left(x_{\lambda}, y_{\lambda}\right)=\mathrm{D}_{t_{\lambda}}^{K, \delta,+}\left(x_{\lambda}\right) \geq c,
$$

we can find a subnet $j \mapsto \lambda(j), j \in J$, such that $j \mapsto y_{\lambda(j)}$ converges to a point $y \in K$ with

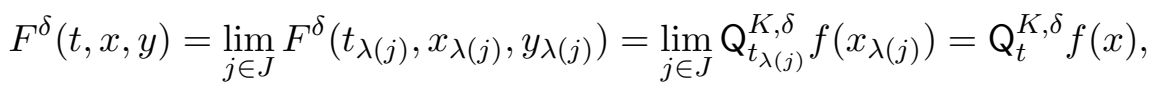

showing that $y \in \mathrm{J}_{t}^{K, \delta} f(x)$. Since

$$
\delta(x, y)=\lim _{j \in J} \delta\left(x_{\lambda(j)}, y_{\lambda(j)}\right) \geq c,
$$

we obtain that $\mathrm{D}_{t}^{K, \delta,+}(x) \geq c$. A similar argument holds for the lower semicontinuity of $\mathrm{D}_{t}^{K, \delta,-} f$.

(c) The decreasing property of $\mathrm{Q}$ w.r.t. $\lambda$ is obvious; in particular it yields

$$
\mathrm{Q}_{t}^{K_{\lambda}, \delta} f(x) \geq \mathrm{Q}_{t}^{K, \delta} f(x) .
$$

On the other hand, by the density of $\cup_{\lambda} K_{\lambda}$ in $K$ and the continuity of $F$, for every $y \in J_{t}^{K, \delta} f(x)$ and $\varepsilon>0$ we can find $\lambda_{\varepsilon} \in \Lambda$ and $y_{\varepsilon} \in K_{\lambda_{\varepsilon}}$ such that $F\left(t, x, y_{\varepsilon}\right) \leq F(t, x, y)+\varepsilon$ so that for every $\lambda \succ \lambda_{\varepsilon}$

$$
\mathrm{Q}_{t}^{K_{\lambda}, \delta} f(x) \leq F\left(t, x, y_{\varepsilon}\right) \leq F(t, x, y)+\varepsilon \leq \mathrm{Q}_{t}^{K, \delta} f(x)+\varepsilon .
$$

Since $\varepsilon>0$ is arbitrary we obtain the proof of the claim.

(d) We argue as in the proof of the second claim: we select $y_{\lambda} \in \mathrm{J}_{t}^{K_{\lambda}, \delta} f(x) \subset K_{\lambda}$ so that

$$
F\left(t, x, y_{\lambda}\right)=\mathrm{Q}_{t}^{K_{\lambda}, \delta} f(x), \quad \mathrm{D}_{t}^{K_{\lambda}, \delta,+} f(x)=\delta\left(x, y_{\lambda}\right) .
$$

We can find a subnet $j \mapsto y_{\lambda(j)}, j \in J$, converging to some $y \in K$ with $S:=\lim \sup _{\lambda \in \Lambda} \mathrm{D}_{t}^{K_{\lambda}, \delta,+} f(x)=$ $\lim _{j \in J} \delta\left(x, y_{\lambda(j)}\right)=\delta(x, y)$. It follows that

$$
\mathrm{Q}_{t}^{K, \delta} f(x) \stackrel{(6.16)}{=} \lim _{j \in J} \mathrm{Q}_{t}^{K_{\lambda(j)}, \delta} f(x)=\lim _{j \in J} F\left(t, x, y_{\lambda(j)}\right)=F(t, x, y)
$$

so that $y \in \mathrm{J}_{t}^{K, \delta} f(x)$. This yields

$$
\mathrm{D}_{t}^{K, \delta,+} f(x) \geq \delta(x, y)=\underset{\lambda \in \Lambda}{\limsup } \mathrm{D}_{t}^{K_{\lambda}, \delta,+} f(x) .
$$

We consider now the behaviour of $\mathrm{Q}^{\delta}=\mathrm{Q}^{X, \delta}$ with respect to $\delta$.

Proposition 6.3. Let $\left(\mathrm{d}_{i}\right)_{i \in I}$ be a directed family of continuous semidistances as in (2.24a,b,c,d) and let $f \in \mathrm{C}_{b}(X)$. For every $x \in X$ the net $i \mapsto \mathrm{Q}_{t}^{\mathrm{d}_{i}} f(x)$ is monotonically converging to $\mathrm{Q}_{t} f(x)$ and

$$
\limsup _{i \in I} \mathrm{D}_{t}^{\mathrm{d}_{i},+} f(x) \leq \mathrm{D}_{t}^{+} f(x) .
$$

More generally, if $j \mapsto i(j), j \in J$, is an increasing net (but not necessarily a subnet)

$$
\lim _{j \in J} \mathrm{Q}_{t}^{\mathrm{d}_{i(j)}} f(x)=\mathrm{Q}_{t} f(x) \Rightarrow \limsup _{j \in J} \mathrm{D}_{t}^{\mathrm{d}_{i(j)},+} f(x) \leq \mathrm{D}_{t}^{+} f(x)
$$


Proof. Since $i \prec j$ yields $\mathrm{d}_{i} \leq \mathrm{d}_{j}$, it is clear that $i \mapsto \mathrm{Q}_{t}^{\mathrm{d}_{i}} f(x)$ is increasing. For every fixed $t, x, y$ we have $\lim _{i \in I} F^{\mathrm{d}_{i}}(t, x, y)=F^{\mathrm{d}}(t, x, y)$ monotonically. The first statement then follows by a standard application of $\Gamma$-convergence of a family of increasing real functions in a compact set. (6.20) is a particular case of (6.21) for the identity map in the directed set $I$.

Let us now assume that $\lim _{j \in J} \mathrm{Q}_{t}^{\mathrm{d}_{i(j)}} f(x)=\mathrm{Q}_{t} f(x)$ along an increasing net $j \mapsto i(j)$. We can select $y_{j} \in X$ such that

$$
\mathrm{Q}_{t}^{\mathrm{d}_{i(j)}} f(x)=f\left(y_{j}\right)+\frac{1}{q t^{q-1}} \mathrm{~d}_{i(j)}^{q}\left(x, y_{j}\right), \quad \mathrm{D}_{t}^{\mathrm{d}_{i(j)},+} f(x)=\mathrm{d}_{i(j)}\left(x, y_{j}\right) .
$$

We can find a further subnet $h \mapsto j(h), h \in H$, such that $\left(y_{j(h)}\right)_{h \in H}$ is convergent to $y \in X$ and

$$
\underset{j \in J}{\limsup } \mathrm{D}_{t}^{\mathrm{d}_{i(j)},+} f(x)=\lim _{h \in H} \mathrm{~d}_{i(j(h))}\left(x, y_{j(h)}\right) .
$$

Passing to the limit in the first equation of (6.22) and using the assumption of (6.21) we get

$$
\mathrm{Q}_{t} f(x)=f(y)+\frac{1}{q t^{q-1}} \lim _{h \in H} \mathrm{~d}_{i(j(h))}^{q}\left(x, y_{j(h)}\right) \stackrel{(2.25)}{\geq} f(y)+\frac{1}{q t^{q-1}} \mathrm{~d}^{q}(x, y) \geq \mathrm{Q}_{t} f(x)
$$

where the last inequality follows by the very definition of $\mathrm{Q}_{t} f(x)$. We deduce that

$$
y \in \mathrm{J}_{t} f(x), \quad \lim _{h \in H} \mathrm{~d}_{i(j(h))}^{q}\left(x, y_{j(h)}\right)=\mathrm{d}^{q}(x, y) .
$$

Since $\mathrm{d}(x, y) \leq \mathrm{D}_{t}^{+} f(x)$, by (6.23) we get (6.21).

Notice that the upper semicontinuity property of (6.20) and of (6.21) are not immediately obvious as in the case of, e.g., (6.16), since $d$ is typically just lower semicontinuous along $\tau$-converging sequences. In the proof we used in an essential way the minimality of $y_{j}$ and the continuity of $f$.

We conclude this section with the main structural properties for the Hopf-Lax evolution generated by d.

Theorem 6.4. Let $f \in \operatorname{Lip}_{b}(X, \tau, \mathrm{d})$ and let $\mathrm{Q}_{t} f, \mathrm{~J}_{t} f, \mathrm{D}_{t}^{ \pm} f$ be defined as (6.4), (6.5), (6.6) for $t>0$.

(a) The functions $(x, t) \mapsto \mathrm{Q}_{t} f(x), \mathrm{D}_{t}^{-} f(x)$ are lower semicontinuous in $X \times(0,+\infty)$ and

$$
\min _{X} f \leq Q_{t} f(x) \leq \max _{X} f \quad \text { for every } t>0, x \in X .
$$

(b) For every $x \in X$

$$
\lim _{t \downarrow 0} \mathrm{Q}_{t} f(x)=\mathrm{Q}_{0} f(x):=f(x),
$$

the map $t \mapsto Q_{t} f(x)$ is Lipschitz in $[0, \infty)$ and satisfies

$$
\frac{\mathrm{d}}{\mathrm{d} t} \mathrm{Q}_{t} f(x)=-\frac{1}{p}\left(\frac{\mathrm{D}_{t}^{ \pm} f(x)}{t}\right)^{q} \text { for } t>0 \text { with at most countable exceptions. }
$$

(c) For every $x \in X$ and $t>0$

$$
\begin{gathered}
f(x)-\mathrm{Q}_{t} f(x)=\frac{t}{p} \int_{0}^{1}\left(\frac{\mathrm{D}_{t r}^{+} f(x)}{t r}\right)^{p} \mathrm{~d} r \\
\limsup _{t \downarrow 0} \frac{f(x)-\mathrm{Q}_{t} f(x)}{t} \leq \frac{1}{p}|\mathrm{D} f|^{p}(x) \leq \frac{1}{p}(\operatorname{lip} f(x))^{p} .
\end{gathered}
$$


Proof. (a) Lower semicontinuity of $\mathrm{Q} f$ is a consequence of the joint lower semicontinuity of $F$ and of the compactness of $(X, \tau)$; it can also be obtained by Proposition 6.3, which characterizes $Q_{t} f$ as a supremum of continuous functions. The bound (6.26) is immediate.

(b) As for the proof of (6.14), we get from (6.9) and (6.13)

$$
\frac{1}{p}\left(\frac{\mathrm{D}_{s}^{+} f(x)}{t}\right)^{q}(t-s) \leq \mathrm{Q}_{s} f(x)-\mathrm{Q}_{t} f(x) \leq \frac{1}{p}\left(\frac{\mathrm{D}_{t}^{-} f(x)}{s}\right)^{q}(t-s),
$$

and (6.8) yields the uniform bound

$$
\left(\frac{\mathrm{D}_{t}^{+} f(x)}{t}\right)^{q} \leq(q \operatorname{Lip}(f, X))^{p}
$$

Since $t \mapsto Q_{t} f(x)$ is decreasing, we obtain

$$
\frac{\left|Q_{s} f(x)-Q_{t} f(x)\right|}{|t-s|} \leq \frac{1}{p}\left(\frac{t}{s}\right)^{q}(q \operatorname{Lip}(f, X))^{p} \quad \text { for every } 0<s<t .
$$

(6.33) shows that $t \mapsto \mathrm{Q}_{t} f(x)$ is Lipschitz in every compact interval of $(0, \infty)$ with

$$
\left|\frac{\mathrm{d}}{\mathrm{d} t} \mathrm{Q}_{t} f(x)\right| \leq \frac{1}{p}(q \operatorname{Lip}(f, X))^{p} \quad \text { for a.e. } t>0
$$

so that $t \mapsto \mathrm{Q}_{t} f(x)$ is Lipschitz in $(0,+\infty)$. In order to prove (6.27) we simply observe that for every $x^{\prime} \in \mathrm{J}_{t} f(x), x^{\prime} \neq x$

$$
f(x)-\mathrm{Q}_{t} f(x)=f(x)-f\left(x^{\prime}\right)-\frac{\mathrm{d}^{q}\left(x, x^{\prime}\right)}{q t^{q-1}} \leq \mathrm{d}\left(x, x^{\prime}\right)\left(\frac{f(x)-f\left(x^{\prime}\right)}{\mathrm{d}\left(x, x^{\prime}\right)}-\frac{1}{q} \frac{\mathrm{d}^{q-1}\left(x, x^{\prime}\right)}{t^{q-1}}\right)
$$

so that

$$
0 \leq f(x)-\mathrm{Q}_{t} f(x) \leq \mathrm{D}_{t}^{-} f(x) \operatorname{Lip}(f, X)
$$

and the right hand side vanishes as $t \downarrow 0$ thanks to (6.32).

(6.28) follows from (6.37) and the monotonicity property (a consequence of (6.9))

$$
\mathrm{D}_{s}^{-} f(x) \leq \mathrm{D}_{s}^{+} f(x) \leq \mathrm{D}_{t}^{-} f(x) \text { for every } x \in X, 0<s<t,
$$

which in particular shows that $\mathrm{D}_{t}^{-} f(x)=\mathrm{D}_{t}^{+} f(x)$ for every $t>0$ with at most countable exceptions.

(c) (6.29) follows by integrating (6.28).

Dividing (6.35) by $t$ we get for every $x^{\prime} \in J_{t} f(x) \backslash\{x\}$

$$
\frac{f(x)-\mathrm{Q}_{t} f(x)}{t}=\frac{\mathrm{d}\left(x, x^{\prime}\right)}{t} \frac{f(x)-f\left(x^{\prime}\right)}{\mathrm{d}\left(x, x^{\prime}\right)}-\frac{1}{q} \frac{\mathrm{d}^{q}\left(x, x^{\prime}\right)}{t^{q}} \leq \frac{1}{p}\left(\frac{f(x)-f\left(x^{\prime}\right)}{\mathrm{d}\left(x, x^{\prime}\right)}\right)^{p} ;
$$

passing to the limit as $t \downarrow 0$ and observing that $\lim _{t \downarrow 0} \mathrm{D}_{t}^{+} f(x)=0$ we obtain (6.30).

We conclude this section with a discussion of the measurability properties of the maps $\mathrm{D}^{ \pm} f$. In the case when $d$ is continuous, $\mathrm{D}^{+} f$ (resp. $\mathrm{D}^{-} f$ ) is upper- (resp. lower-) semicontinuous by Proposition 6.3. In the general case we can anyway prove that they are $\mathfrak{m} \times \mathscr{L}^{1}$ measurable. 
Lemma 6.5 (Conditional semicontinuity and measurability of $\mathrm{D}^{ \pm} f$ ). Under the same assumptions of Theorem 6.4:

(a) for every net $\left(x_{\lambda}, t_{\lambda}\right)_{\lambda \in \Lambda}$ in $X \times(0, \infty)$ such that $\lim _{\lambda \in \Lambda}\left(x_{\lambda}, t_{\lambda}\right)=(x, t) \in X \times(0, \infty)$ we have

$$
\lim _{\lambda \in \Lambda} Q_{t_{\lambda}} f\left(x_{\lambda}\right)=Q_{t} f(x) \Rightarrow\left\{\begin{array}{l}
\limsup _{\lambda \in \Lambda} D_{t_{\lambda}}^{+} f\left(x_{\lambda}\right) \leq \mathrm{D}_{t}^{+} f(x), \\
\liminf _{\lambda \in \Lambda} \mathrm{D}_{t_{\lambda}}^{-} f\left(x_{\lambda}\right) \geq \mathrm{D}_{t}^{-} f(x) .
\end{array}\right.
$$

(b) The maps $(x, t) \mapsto \mathrm{D}_{t}^{ \pm} f(x)$ are Lusin $\mathfrak{m} \otimes \mathscr{L}^{1}$-measurable in $X \times(0, \infty)$; moreover, for every $t>0$ the maps $x \mapsto \mathrm{D}_{t}^{ \pm} f(x)$ are Lusin $\mathfrak{m}$-measurable in $X$.

Proof. (a) Let us check the upper semicontinuity of $\mathrm{D}^{+} f$, by arguing as in the proof of Lemma 6.2(b) (the proof of the conditional lower semicontinuity of $\mathrm{D}^{-} f$ is completely analogous). We fix $c<\mathrm{D}_{t}^{+} f(x)$ and we suppose that for some $\lambda_{0} \in \Lambda \mathrm{D}_{t_{\lambda}}^{+}\left(x_{\lambda}\right) \geq c$ for every $\lambda \succ \lambda_{0}$. We pick $y_{\lambda} \in \mathrm{J}_{t_{\lambda}}\left(x_{\lambda}\right)$ such that

$$
F\left(t_{\lambda}, x_{\lambda}, y_{\lambda}\right)=\mathrm{Q}_{t_{\lambda}} f\left(x_{\lambda}\right), \quad \mathrm{d}\left(x_{\lambda}, y_{\lambda}\right)=\mathrm{D}_{t_{\lambda}}^{+}\left(x_{\lambda}\right) \geq c .
$$

We can find a subnet $j \mapsto \lambda(j), j \in J$, such that $j \mapsto y_{\lambda(j)}$ converges to a point $y \in X$ with

$$
F(t, x, y) \leq \liminf _{j \in J} F\left(t_{\lambda(j)}, x_{\lambda(j)}, y_{\lambda(j)}\right)=\liminf _{j \in J} Q_{t_{\lambda(j)}} f\left(x_{\lambda(j)}\right) \stackrel{(6.38)}{=} \mathrm{Q}_{t} f(x),
$$

showing that $y \in \mathrm{J}_{t} f(x)$ and $\lim _{j \in J} \mathrm{~d}\left(x_{\lambda(j)}, y_{\lambda(j)}\right)=\mathrm{d}(x, y) \geq c$. It follows that $\mathrm{D}_{t}^{+} f(x) \geq$ $\mathrm{d}(x, y) \geq c$.

(b) Since the map $\mathrm{Q} f$ is lower semicontinuous, it is Lusin $\mathfrak{m} \otimes \mathscr{L}^{1}$ measurable in $X \times(0, \infty)[57$, I.1.5, Theorem 5]. For every compact set $K \subset(0, \infty)$ and every $\varepsilon>0$ we can find a compact subset $H_{\varepsilon} \subset X \times K$ such that the restriction of $\mathrm{Q} f$ to $H_{\varepsilon}$ is continuous and $\mathfrak{m} \otimes \mathscr{L}^{1}\left((X \times K) \backslash H_{\varepsilon}\right) \leq \varepsilon / 2$. By the previous claim, we deduce that the restriction of $\mathrm{D}^{ \pm} f$ to $H_{\varepsilon}$ are semicontinuous, and thus Lusin $\mathfrak{m} \otimes \mathscr{L}^{1}$-measurable: therefore we can find a further compact subset $H_{\varepsilon}^{\prime} \subset H_{\varepsilon}$ such that the restriction of $\mathrm{D}^{ \pm} f$ to $H_{\varepsilon}^{\prime}$ are continuous and $\mathfrak{m} \otimes \mathscr{L}^{1}\left(H_{\varepsilon} \backslash H_{\varepsilon}^{\prime}\right) \leq \varepsilon / 2$, so that $\mathfrak{m} \otimes \mathscr{L}^{1}\left((X \times K) \backslash H_{\varepsilon}^{\prime}\right) \leq \varepsilon$. We conclude that $\mathrm{D}^{ \pm} f$ are Lusin $\mathfrak{m} \otimes \mathscr{L}^{1}$-measurable. The second statement can be proved by the same argument.

\subsection{Invariance of the Cheeger energy with respect to $\mathscr{A}$ when $(X, \tau)$ is compact}

As a preliminary obvious remark, we observe that if $\mathscr{A}^{\prime} \subset \mathscr{A}^{\prime \prime} \subset \operatorname{Lip}_{b}(X, \tau, \mathrm{d})$ are two algebras of Lipschitz functions compatible with the metric-topological measure structure $\mathbb{X}=(X, \tau, \mathrm{d}$, m) we have

$$
H^{1, p}\left(\mathbb{X}, \mathscr{A}^{\prime}\right) \subset H^{1, p}\left(\mathbb{X}, \mathscr{A}^{\prime \prime}\right) \subset H^{1, p}(\mathbb{X}),
$$

and for every $f \in H^{1, p}\left(\mathbb{X}, \mathscr{A}^{\prime}\right)$

$$
\mathrm{CE}_{p, \mathscr{A}^{\prime}}(f) \geq \mathrm{CE}_{p, \mathscr{A}^{\prime \prime}}(f) \geq \mathrm{CE}_{p}(f), \quad|\mathrm{D} f|_{\star, \mathscr{A} \prime^{\prime}} \geq|\mathrm{D} f|_{\star, \mathscr{A ^ { \prime \prime }}} \geq|\mathrm{D} f|_{\star} \quad \text { m-a.e. in } X .
$$

We will see that (6.40) and (6.41) can be considerably refined, obtaining the complete independence of the choice of $\mathscr{A}$. In this section we will focus on the case when $(X, \tau)$ is a compact topological space; we suppose that $\mathscr{A}$ is an algebra compatible with $\mathbb{X}$, we denote by $I$ the directed set of all the finite collections $i \subset \mathscr{A}_{1}$ satisfying $f \in i \Rightarrow-f \in i$ and we set

$$
\mathrm{d}_{i}(x, y):=\sup _{f \in i} f(x)-f(y), \quad i \text { is a finite subset of } \mathscr{A}_{1} \text { satisfying } f \in i \Rightarrow-f \in i .
$$


Lemma 6.6. Let us suppose that $(X, \tau)$ is compact and $\mathscr{A}$ is an algebra compatible with $\mathbb{X}$ generating the bounded continuous semidistances $\left(\mathrm{d}_{i}\right)_{i \in I}$ as in (6.42).

(a) For every $y \in X$ and $i \in I$ the map $h_{i}^{y}: x \rightarrow \mathrm{d}_{i}(x, y)$ belongs to $H^{1, p}(\mathbb{X}, \mathscr{A})$ and $\left|\mathrm{D} h_{i}^{y}\right|_{\star, \mathscr{A}} \leq$ $1 \mathfrak{m}$-a.e.

(b) For every $f \in \mathrm{C}(X), t>0, i \in I$, the function $\mathrm{Q}_{t}^{\mathrm{d}_{i}} f$ belongs to $H^{1, p}(\mathbb{X}, \mathscr{A})$ and

$$
\left|\mathrm{DQ}_{t}^{\mathrm{d}_{i}} f\right|_{\star, \mathscr{A}} \leq t^{-1} \mathrm{D}_{t}^{\mathrm{d}_{i},+} f \quad \mathfrak{m} \text {-a.e.. }
$$

(c) For every $f \in \mathrm{C}(X)$ and $t>0$ the function $\mathrm{Q}_{t} f$ belongs to $H^{1, p}(\mathbb{X}, \mathscr{A})$ and

$$
\left|\mathrm{DQ}_{t} f\right|_{\star, \mathscr{A}} \leq t^{-1} \mathrm{D}_{t}^{+} f \quad \mathfrak{m} \text {-a.e. }
$$

Proof. Claim (a) immediately follows from Corollary 5.13.

(b) Let us denote by $\Pi$ the directed family of finite subset of $X$. For every $\pi \in \Pi$, the definition of $\mathrm{Q}_{t}^{\pi, \mathrm{d}_{i}}$ given in (6.3), the chain rule (5.16), the previous claim, and Corollary 5.13 yield

$$
\mathrm{Q}_{t}^{\pi, \mathrm{d}_{i}} f \in H^{1, p}(\mathbb{X}, \mathscr{A}), \quad\left|\mathrm{DQ}_{t}^{\pi, \mathrm{d}_{i}} f\right|_{\star, \mathscr{A}} \leq g_{t}^{\pi, \mathrm{d}_{i}}:=\left(t^{-1} \mathrm{D}_{t}^{\pi, \mathrm{d}_{i}+} f\right)^{q-1}
$$

By Lemma 6.2(c), for every $x \in X$ and $t>0 \pi \mapsto \mathrm{Q}_{t}^{\pi, \mathrm{d}_{i}} f(x)$ is a decreasing net, converging to $\mathrm{Q}_{t}^{\mathrm{d}_{i}} f(x)$. Thanks to 2.8 and to the uniform bound (6.7) (where $S:=\max _{x, y \in X} \mathrm{~d}_{i}(x, y)$ ) we have

$$
\lim _{\pi \in \Pi} \int_{X}\left|\mathrm{Q}_{t}^{\pi, \mathrm{d}_{i}} f-\mathrm{Q}_{t}^{\mathrm{d}_{i}} f\right| \mathrm{d} \mathfrak{m}=\lim _{\pi \in \Pi} \int_{X} \mathrm{Q}_{t}^{\pi, \mathrm{d}_{i}} f \mathrm{~d} \mathfrak{m}-\int_{X} \mathrm{Q}_{t}^{\mathrm{d}_{i}} f \mathrm{~d} \mathfrak{m}=0 .
$$

We can thus find an increasing sequence $n \mapsto \pi_{n} \in \Pi$ such that

$$
\lim _{n \rightarrow \infty} \int_{X}\left|\mathrm{Q}_{t}^{\pi_{n}, \mathrm{~d}_{i}} f-\mathrm{Q}_{t}^{\mathrm{d}_{i}} f\right| \mathrm{d} \mathfrak{m}=\lim _{n \rightarrow \infty} \int_{X}\left|\mathrm{Q}_{t}^{\pi_{n}, \mathrm{~d}_{i}} f-\mathrm{Q}_{t}^{\mathrm{d}_{i}} f\right|^{p} \mathrm{~d} \mathfrak{m}=0
$$

and a m-negligible subset $N \subset X$ such that

$$
\lim _{n \rightarrow \infty} \mathrm{Q}_{t}^{\pi_{n}, \mathrm{~d}_{i}} f(x)=\mathrm{Q}_{t}^{\mathrm{d}_{i}} f(x) \text { for every } x \in X \backslash N .
$$

Applying (6.16) we deduce

$$
\limsup _{n \rightarrow \infty} g_{t}^{\pi_{n}, \mathrm{~d}_{i}}(x) \leq g_{t}^{\mathrm{d}_{i}}(x):=\left(t^{-1} \mathrm{D}_{t}^{\mathrm{d}_{i}+} f\right)^{q-1} \quad \text { for every } x \in X \backslash N .
$$

By (6.45), it follows that any weak limit point in $L^{p}(X, \mathfrak{m})$ of the (uniformly bounded) sequence $\left(\left|\mathrm{DQ}_{t}^{\pi_{n}, \mathrm{~d}_{i}} f\right|_{\star, \mathscr{A}}\right)_{n \in \mathbb{N}}$ will be bounded by $t^{-1} \mathrm{D}_{t}^{\mathrm{d}_{i}+} f$. By (6.46) we obtain (6.43).

(c) The argument is very similar to the previous one, but now using Proposition 6.3 and the net $i \mapsto \mathrm{d}_{i}$.

Theorem 6.7. If $(X, \tau)$ is a compact space and $\mathscr{A} \subset \operatorname{Lip}(X, \tau, \mathrm{d})$ is a compatible algebra according to definition 2.17, then $H^{1, p}(\mathbb{X})=H^{1, p}(\mathbb{X}, \mathscr{A})$ with equal minimal relaxed gradient (and therefore equal Cheeger energy). Equivalently, for every $f \in H^{1, p}(\mathbb{X})$ there exists a sequence $f_{n} \in \mathscr{A}$ such that

$$
f_{n} \rightarrow f, \quad \operatorname{lip} f_{n} \rightarrow|\mathrm{D} f|_{\star} \quad \text { strongly in } L^{p}(X, \mathfrak{m}) .
$$


Proof. Let us denote by $|\mathrm{D} f|_{\star, \mathscr{A}}$ (resp. $|\mathrm{D} f|_{\star}$ ) the minimal relaxed gradient induced by $\mathscr{A}$ (resp. by $\operatorname{Lip}(X, \tau, \mathrm{d}))$. It is clear that $H^{1, p}(X, \mathscr{A}) \subset H^{1, p}(X)$ and $|\mathrm{D} f|_{\star} \leq|\mathrm{D} f|_{\star, \mathscr{A}}$ for every $f \in H^{1, p}(X, \mathscr{A})$; in order to prove the Theorem it is sufficient to show that every $f \in \operatorname{Lip}(X, \tau, \mathrm{d})$ belongs to $H^{1, p}(\mathbb{X}, \mathscr{A})$ with $|\mathrm{D} f|_{\star, \mathscr{A}} \leq \operatorname{lip} f \mathfrak{m}$-a.e.

We select an arbitrary Borel set $B \subset X$; by using the uniform bound (a consequence of (6.34))

$$
\frac{f(x)-Q_{t} f(x)}{t} \leq(q \operatorname{Lip}(f, X))^{p} \quad \text { for every } x \in X,
$$

the superior limit (6.30) and Fatou's Lemma, we obtain

$$
\frac{1}{p} \int_{B}(\operatorname{lip} f(x))^{p} \mathrm{~d} \mathfrak{m}(x) \geq \limsup _{t \downarrow 0} \int_{B} \frac{f(x)-Q_{t} f(x)}{t} \mathrm{~d} \mathfrak{m}(x) .
$$

On the other hand, (6.29), the measurability of $\mathrm{D}^{+} f$ given by Lemma 6.5, and Fubini's Theorem yield

$$
\begin{aligned}
\int_{B} \frac{f(x)-\mathrm{Q}_{t} f(x)}{t} \mathrm{~d} \mathfrak{m}(x) & =\frac{1}{p} \int_{B \times(0,1)}\left(\frac{\mathrm{D}_{t r}^{+} f(x)}{t r}\right)^{p} \mathrm{~d}\left(\mathfrak{m} \otimes \mathscr{L}^{1}\right)(x, r) \\
& =\frac{1}{p} \int_{0}^{1}\left(\int_{B}\left(\frac{\mathrm{D}_{t r}^{+} f(x)}{t r}\right)^{p} \mathrm{~d} \mathfrak{m}(x)\right) \mathrm{d} r .
\end{aligned}
$$

A further application of Fatou's Lemma yields

$$
\liminf _{t \downarrow 0} \int_{B} \frac{f(x)-Q_{t} f(x)}{t} \mathrm{~d} \mathfrak{m}(x) \geq \frac{1}{p} \liminf _{s \downarrow 0} \int_{B}\left(\frac{\mathrm{D}_{s}^{+} f(x)}{s}\right)^{p} \mathrm{~d} \mathfrak{m}(x),
$$

where we used the fact that for every $r \in(0,1)$

$$
\liminf _{t \downarrow 0} \int_{B}\left(\frac{\mathrm{D}_{t r}^{+} f(x)}{t r}\right)^{p} \mathrm{~d} \mathfrak{m}(x)=\liminf _{s \downarrow 0} \int_{B}\left(\frac{\mathrm{D}_{s}^{+} f(x)}{s}\right)^{p} \mathrm{~d} \mathfrak{m}(x) .
$$

Recalling (6.43) and the fact that $\mathrm{Q}_{s} f \rightarrow f$ in $L^{p}(X, \mathfrak{m})$ as $s \downarrow 0$ we get

$$
\liminf _{s \downarrow 0} \int_{B}\left(\frac{\mathrm{D}_{s}^{+} f}{s}\right)^{p} \mathrm{~d} \mathfrak{m} \geq \liminf _{s \downarrow 0} \int_{B}\left|\mathrm{DQ} Q_{s} f\right|_{\star, \mathscr{A}}^{p} \mathrm{~d} \mathfrak{m} \geq \int_{B}|\mathrm{D} f|_{\star, \mathscr{A}}^{p} \mathrm{~d} \mathfrak{m} .
$$

Combining (6.51), (6.52) and (6.53) we deduce that

$$
\int_{B}(\operatorname{lip} f(x))^{p} \mathrm{~d} \mathfrak{m}(x) \geq \int_{B}|\mathrm{D} f|_{\star, \mathscr{A}}^{p}(x) \mathrm{d} \mathfrak{m}(x)
$$

for every Borel subset $B$, so that $\operatorname{lip} f(x) \geq|\mathrm{D} f|_{\star, \mathscr{A}}(x)$ for $\mathfrak{m}$-a.e. $x \in X$.

\subsection{Notes}

$\S 6.1$ collects all the basic estimates concerning the Hopf-Lax flow in a general extended metric-topological setting. We followed the approach of $[9, \S 3]$ with some differences: we assumed compactness of $(X, \tau)$ (as in $[8,40])$, we considered general exponents $q=p^{\prime} \in(1, \infty)$ as in [8], and we devoted some effort to study the dependence of the Hopf-Lax formula on the distance and on the minimizing set, a point of view that has also been used in [12,3]. In this respect, compactness plays an essential role. Differently from [9], the HopfLax flow is not used as a crucial ingredient for the so-called Kuwada Lemma [9, $\S$ 6], [48], but as a powerful approximation tool of general functions by elements in the algebra $\mathscr{A}$. 
$\S 6.2$ contains the crucial results which justify the study of the Hopf-Lax formula in our setting: Lemma 6.6 provides a crucial estimate of $\left|D Q_{t} f\right|_{\star, \mathscr{A}}$ for the regularized functions and Theorem 6.7 shows that compatible algebra are dense (in energy) in $H^{1, p}(\mathbb{X})$. Both the results are inspired by the techniques of [12, Theorem 3.12] and of $[3, \S 12]$.

\section{Part III}

\section{$p$-Modulus and nonparametric dynamic plans}

Assumption. As in the previous section, we consider an extended metric-topological measure space $\mathbb{X}=(X, \tau, \mathrm{d}, \mathfrak{m})$ and we fix an exponent $p \in(1,+\infty) \cdot \operatorname{RA}(X)=\mathrm{RA}(X, \mathrm{~d})$ is the space of rectifiable arcs with the quotient topology $\tau_{\mathrm{A}}$ studied in $\S 3.2$, see Proposition 3.6.

\section{$7 \quad p$-Modulus of a family of measures and of a family of rectifiable arcs}

\section{1 $p$-Modulus of a family of Radon measures}

Given $\Sigma \subset \mathcal{M}_{+}(X)$ we define (with the usual convention $\inf \emptyset=\infty$ )

$$
\begin{gathered}
\operatorname{Mod}_{p}(\Sigma):=\inf \left\{\int_{X} f^{p} \mathrm{dm}: f \in \mathcal{L}_{+}^{p}(X, \mathfrak{m}), \int_{X} f \mathrm{~d} \mu \geq 1 \text { for all } \mu \in \Sigma\right\}, \\
\operatorname{Mod}_{p, c}(\Sigma):=\inf \left\{\int_{X} f^{p} \mathrm{~d} \mathfrak{m}: f \in \mathrm{C}_{b}(X), \int_{X} f \mathrm{~d} \mu \geq 1 \text { for all } \mu \in \Sigma\right\} .
\end{gathered}
$$

Since the infimum in (7.2) is unchanged if we restrict the minimization to nonnegative functions $f \in \mathrm{C}_{b}(X)$ we get $\operatorname{Mod}_{p, c}(\Sigma) \geq \operatorname{Mod}_{p}(\Sigma)$. Also, whenever $\Sigma$ contains the null measure, we have $\operatorname{Mod}_{p, c}(\Sigma)=\operatorname{Mod}_{p}(\Sigma)=\infty$, whereas $\operatorname{Mod}_{p, c}(\emptyset)=0$.

Definition 7.1 $\left(\operatorname{Mod}_{p}\right.$-negligible sets and properties $\operatorname{Mod}_{p}$-a.e.). A set $\Sigma \subset \mathcal{M}_{+}(X)$ is said to be $\operatorname{Mod}_{p}$-negligible if $\operatorname{Mod}_{p}(\Sigma)=0$.

We say that a property $P$ on $\mathcal{M}_{+}(X)$ holds $\operatorname{Mod}_{p}$-a.e. if the set of measures where $P$ fails is $\operatorname{Mod}_{p^{-}}$ negligible.

The next result collects various well known properties of the Modulus, see e.g. [17], [46, $§ 5.2]$.

Proposition 7.2. The set functions $\Sigma \subset \mathcal{M}_{+}(X) \mapsto \operatorname{Mod}_{p}(\Sigma), \Sigma \subset \mathcal{M}_{+}(X) \mapsto \operatorname{Mod}_{p, c}(\Sigma)$ satisfy the following properties:

(a) both are monotone and subadditive.

(b) If $g \in \mathcal{L}_{+}^{p}(X, \mathfrak{m})$ then $\int_{X} g \mathrm{~d} \mu<\infty$ for $\operatorname{Mod}_{p}$-almost every $\mu$; conversely, if $\operatorname{Mod}_{p}(\Sigma)=0$ then there exists $g \in \mathcal{L}_{+}^{p}(X, \mathfrak{m})$ such that $\int_{X} g \mathrm{~d} \mu=\infty$ for every $\mu \in \Sigma$.

(c) [Fuglede's Lemma] If $\left(f_{n}\right) \subset \mathcal{L}_{+}^{p}(X, \mathfrak{m})$ converges in $L^{p}(X, \mathfrak{m})$ seminorm to $f \in \mathcal{L}_{+}^{p}(X, \mathfrak{m})$, there exists a subsequence $\left(f_{n(k)}\right)$ such that

$$
\lim _{k \rightarrow \infty} \int_{X}\left|f_{n(k)}-f\right| \mathrm{d} \mu=0 \quad \operatorname{Mod}_{p} \text {-a.e. in } \mathcal{M}_{+}(X) .
$$


(d) For every $\Sigma \subset \mathcal{M}_{+}(X)$ with $\operatorname{Mod}_{p}(\Sigma)<\infty$ there exists $f \in \mathcal{L}_{+}^{p}(X, \mathfrak{m})$, unique up to $\mathfrak{m}$ negligible sets, such that $\int_{X} f \mathrm{~d} \mu \geq 1 \operatorname{Mod}_{p}$-a.e. on $\Sigma$ and $\|f\|_{p}^{p}=\operatorname{Mod}_{p}(\Sigma)$.

(e) If $\Sigma_{n}$ are nondecreasing subsets of $\mathcal{M}_{+}(X)$ then $\operatorname{Mod}_{p}\left(\Sigma_{n}\right) \uparrow \operatorname{Mod}_{p}\left(\cup_{n} \Sigma_{n}\right)$.

(f) If $K_{n}$ are nonincreasing compact subsets of $\mathcal{M}_{+}(X)$ then $\operatorname{Mod}_{p, c}\left(K_{n}\right) \downarrow \operatorname{Mod}_{p, c}\left(\cap_{n} K_{n}\right)$.

Proof. We repeat almost word by word the arguments of [2, Proposition 2.2].

(a) Monotonicity is an obvious consequence of the definition. For the subadditivity, if we take two sets $A, B \subset \mathcal{M}_{+}(X)$ and two functions $f, g \in \mathcal{L}_{+}^{p}(X, \mathfrak{m})$ with $\int_{X} f \mathrm{~d} \mu \geq 1$ for every $\mu \in A$ and $\int_{X} g \mathrm{~d} \mu \geq 1$ for every $\mu \in B$, then the function $h:=\left(f^{p}+g^{p}\right)^{1 / p} \geq \max (f, g)$ still satisfies $\int_{X} h \mathrm{~d} \mu \geq 1$ for every $\mu \in A \cup B$, hence

$$
\operatorname{Mod}_{p}(A \cup B) \leq \int_{X} h^{p} \mathrm{~d} \mathfrak{m}=\int_{X} f^{p} \mathrm{~d} \mathfrak{m}+\int_{X} g^{p} \mathrm{~d} \mathfrak{m} .
$$

Minimizing over $f$ and $g$ we get the subadditivity.

(b) If we consider the set where the property fails

$$
\Sigma=\left\{\mu \in \mathcal{M}_{+}(X): \int_{X} g \mathrm{~d} \mu=\infty\right\},
$$

then it is clear that for every $k>0$ we have $\Sigma=\left\{\mu \in \mathcal{M}_{+}(X): \int_{X} k g \mathrm{~d} \mu=\infty\right\}$ so that $\operatorname{Mod}_{p}(\Sigma) \leq$ $k^{p}\|g\|_{p}^{p}$ for every $\kappa>0$ and we deduce that $\operatorname{Mod}_{p}(\Sigma)=0$.

Conversely, if $\operatorname{Mod}_{p}(A)=0$ for every $n \in \mathbb{N}$ we can find $g_{n} \in \mathcal{L}_{+}^{p}(X, \mathfrak{m})$ with $\int_{X} g_{n} \mathrm{~d} \mu \geq 1$ for every $\mu \in A$ and $\int_{X} g_{n}^{p} \mathrm{dm} \leq 2^{-n p}$. Thus $g:=\sum_{n} g_{n}$ satisfies the required properties.

(c) Let $f_{n(k)}$ be a subsequence such that $\left\|f-f_{n(k)}\right\|_{p} \leq 2^{-k}$. If we set

$$
g(x):=\sum_{k=1}^{\infty}\left|f(x)-f_{n(k)}(x)\right|
$$

we have that $g \in \mathcal{L}_{+}^{p}(X, \mathfrak{m})$ and $\|g\|_{L^{p}(X, \mathfrak{m})} \leq 1$; in particular we have, for Claim (b) above, that $\int_{X} g \mathrm{~d} \mu$ is finite for $\operatorname{Mod}_{p}$-almost every $\mu$. For those $\mu$ we get

$$
\sum_{k=1}^{\infty} \int_{X}\left|f-f_{n(k)}\right| \mathrm{d} \mu<\infty
$$

which yields (7.3).

(d) Claim (b) shows in particular that

$$
\operatorname{Mod}_{p}(\Sigma)=\inf \left\{\int_{X} f^{p} \mathrm{dm}: \int_{X} f \mathrm{~d} \mu \geq 1 \text { for } \operatorname{Mod}_{p} \text {-a.e. } \mu \in \Sigma\right\}
$$

so that by Claim (c) the class of admissible functions $f$ involved in the variational definition od $\operatorname{Mod}_{p}$ is a convex and closed subset of the Lebesgue space $L^{p}(X, \mathfrak{m})$. Hence, uniqueness follows by the strict convexity of the $L^{p}$-norm.

(e) By the monotonicity, it is clear that $\operatorname{Mod}_{p}\left(A_{n}\right)$ is an increasing sequence and that setting $M:=$ $\lim _{n \rightarrow \infty} \operatorname{Mod}_{p}\left(A_{n}\right)$ we have $M \geq \operatorname{Mod}_{p}\left(\cup_{n} A_{n}\right)$. 
If $M=\infty$ there is nothing to prove, otherwise, we need to show that $\operatorname{Mod}_{p}\left(\cup_{n} A_{n}\right) \leq M$; let $\left(f_{n}\right) \subset \mathcal{L}_{+}^{p}(X, \mathfrak{m})$ be a sequence of functions such that $\int_{X} f \mathrm{~d} \mu_{n} \geq 1$ on $A_{n}$ and $\left\|f_{n}\right\|_{L^{p}(X, \mathfrak{m})}^{p} \leq$ $\operatorname{Mod}_{p}\left(A_{n}\right)+\frac{1}{n}$. In particular we get that $\limsup _{n}\left\|f_{n}\right\|_{p}^{p}=M<\infty$ and so, possibly extracting a subsequence, we can assume that $f_{n}$ weakly converge to some $f \in \mathcal{L}_{+}^{p}(X, \mathfrak{m})$. By Mazur lemma we can find convex combinations

$$
\hat{f}_{n}=\sum_{k=n}^{\infty} \kappa_{k, n} f_{k}
$$

such that $\hat{f}_{n}$ converge strongly to $f$ in $L^{p}(X, \mathfrak{m})$; furthermore we have that $\int_{X} f_{k} \mathrm{~d} \mu \geq 1$ on $A_{n}$ if $k \geq n$ and so

$$
\int_{X} \hat{f}_{n} \mathrm{~d} \mu=\sum_{k=n}^{\infty} \kappa_{k, n} \int_{X} f \mathrm{~d} \mu_{k} \geq 1 \quad \text { on } A_{n} .
$$

By Claim (c) above we obtain a subsequence $n(k)$ and a $\operatorname{Mod}_{p}$-negligible set $\Sigma \subset \mathcal{M}_{+}(X)$ such that $\int_{X} \hat{f}_{n(k)} \mathrm{d} \mu \rightarrow \int_{X} f \mathrm{~d} \mu$ outside $\Sigma$; in particular $\int_{X} f \mathrm{~d} \mu \geq 1$ on $\cup_{n} A_{n} \backslash \Sigma$.

By Claim (b) we can find $g \in \mathcal{L}_{+}^{p}(X, \mathfrak{m})$ such that $\int_{X} g \mathrm{~d} \mu=\infty$ on $\Sigma$, so that we have $\int_{X}(f+$ $\varepsilon g) \mathrm{d} \mu \geq 1$ on $\cup_{n} A_{n}$ and

$$
\operatorname{Mod}_{p}\left(\cup_{n} A_{n}\right)^{1 / p} \leq\|\varepsilon g+f\|_{p} \leq \varepsilon\|g\|_{p}+\|f\|_{p} \leq \varepsilon\|g\|+\liminf \left\|f_{n}\right\|_{p} \leq \varepsilon\|g\|_{p}+M^{1 / p} .
$$

Letting $\varepsilon \rightarrow 0$ and taking the $p$-th. power the inequality $\operatorname{Mod}_{p}(A) \leq \sup _{n} \operatorname{Mod}_{p}\left(A_{n}\right)$ follows.

(f) By the monotonicity we get $\operatorname{Mod}_{p, c}(K) \leq \operatorname{Mod}_{p, c}\left(K_{n}\right)$; if $C:=\lim _{n \rightarrow \infty} \operatorname{Mod}_{p, c}\left(K_{n}\right)$, we only have to prove $\operatorname{Mod}_{p, c}(K) \geq C$ and it is not restrictive to assume $C>0$. We argue by contradiction: if $\operatorname{Mod}_{p, c}(K)<C$ we can find a nonnegative $\psi \in \mathrm{C}_{b}(X)$ such that $\int_{X} \psi \mathrm{d} \mu \geq 1$ for every $\mu \in K$ and $\alpha^{p}=\int_{X} \psi^{p} \mathrm{dm}<C$. Setting $\phi:=\alpha^{-1} \psi$ we obtain a function $\phi \in \mathrm{C}_{b}(X)$ with $\|\phi\|_{p}=1$ and

$$
\inf _{\mu \in K} \int_{X} \phi \mathrm{d} \mu>\alpha^{-1}
$$

By the compactness of $K$ the infimum above is a minimum; since $K_{n}$ is decreasing,

$$
\min _{\mu \in K_{n}} \int_{X} \phi \mathrm{d} \mu \rightarrow \min _{\mu \in K} \int_{X} \phi \mathrm{d} \mu
$$

It follows that there exists $\bar{n} \in \mathbb{N}$ such that $\min _{\mu \in K_{n}} \int_{X} \phi \mathrm{d} \mu \geq \alpha^{-1}$ for every $n \geq \bar{n}$ so that using $\psi=\alpha \phi$ we deduce that $\operatorname{Mod}_{p, c}\left(K_{n}\right) \leq \alpha^{p}<C$ for $n \geq \bar{n}$, a contradiction.

Another important property is the tightness of $\operatorname{Mod}_{p}$ in $\mathcal{M}_{+}(X)$.

Lemma 7.3 (Tightness of $\left.\operatorname{Mod}_{p}\right)$. For every $\varepsilon>0$ there exists $K_{\varepsilon} \subset \mathcal{M}_{+}(X)$ compact such that $\operatorname{Mod}_{p}\left(\mathcal{M}_{+}(X) \backslash K_{\varepsilon}\right) \leq \varepsilon$.

Proof. Since $\mathfrak{m}$ is a Radon measure we can find an nondecreasing family of $\tau$-compact sets $K_{n} \subset X$ such that $m_{n}=\mathfrak{m}\left(X \backslash K_{n}\right)>0, \lim _{n \rightarrow \infty} \mathfrak{m}\left(X \backslash K_{n}\right)=0$. We set

$$
\delta_{n}=\left(\sqrt{m_{n}}+\sqrt{m_{n+1}}\right)^{1 / p}, \quad a_{n}:=\delta_{n}^{-1},
$$

observing that $\delta_{n} \downarrow 0$ and $a_{n} \uparrow+\infty$ as $n \rightarrow \infty$. For $k \in \mathbb{N}$ let us now define the sets

$$
E_{k}:=\left\{\mu \in \mathcal{M}_{+}(X): \mu(X) \leq k, \mu(X \backslash K) \leq \delta_{n} \text { for every } n \geq k\right\},
$$


which are compact in $\mathcal{M}_{+}(X)$ by Theorem 2.2.

To evaluate $\operatorname{Mod}_{p}\left(\mathcal{M}_{+}(X) \backslash E_{k}\right)$ we introduce the functions

$$
f_{k}(x):= \begin{cases}0 & \text { if } x \in K_{k}, \\ a_{n} & \text { if } x \in K_{n+1} \backslash K_{n} \text { and } n \geq k, \\ +\infty & \text { otherwise. }\end{cases}
$$

We observe that if $\mu \in \mathcal{M}_{+}(X) \backslash E_{k}$ then we have either $\mu(X)>k$ or $\mu\left(X \backslash K_{n}\right)>\delta_{n}$ for some $n \geq k$. In either case the integral of the function $f_{k}+\frac{1}{k}$ along $\mu$ is greater or equal to 1 :

- if $\mu(X)>k$ then

$$
\int_{X}\left(f_{k}+\frac{1}{k}\right) \mathrm{d} \mu \geq \frac{1}{k} \ell(\mu) \geq 1
$$

- if $\mu\left(X \backslash K_{n}\right)>\delta_{n}$ for some $n \geq k$ we have that

$$
\int_{X}\left(f_{k}+\frac{1}{k}\right) \mathrm{d} \mu \geq \int_{X \backslash K_{n}} f_{k} \mathrm{~d} \mu \geq \int_{X \backslash K_{n}} a_{n} \mathrm{~d} \mu>\delta_{n} a_{n}=1 .
$$

So we have that $\operatorname{Mod}_{p}\left(\mathcal{M}_{+}(X) \backslash E_{k}\right) \leq\left\|f_{k}+\frac{1}{k}\right\|_{p}^{p} \leq\left(\left\|f_{k}\right\|_{p}+\|1 / k\|_{p}\right)^{p}$. But

$$
\begin{aligned}
& \int_{X} f_{k}^{p} \mathrm{~d} \mathfrak{m}=\sum_{n=k}^{\infty} \int_{K_{n+1} \backslash K_{n}} a_{n}^{p} \mathrm{~d} \mathfrak{m}=\sum_{n=k}^{\infty} \frac{m_{n}-m_{n+1}}{\sqrt{m_{n}}+\sqrt{m_{n+1}}}=\sum_{n=k}^{\infty}\left(\sqrt{m_{n}}-\sqrt{m_{n+1}}\right)=\sqrt{m_{k}} \\
& \left\|f_{k}+\frac{1}{k}\right\| \leq\left(m_{k}\right)^{1 /(2 p)}+(\mathfrak{m}(X))^{1 / p} / k
\end{aligned}
$$

and therefore we obtain $\operatorname{Mod}_{p}\left(\mathcal{M}_{+}(X) \backslash E_{k}\right) \leq\left(\left(m_{k}\right)^{1 /(2 p)}+(\mathfrak{m}(X))^{1 / p} / k\right)^{p} \rightarrow 0$.

\section{2 $p$-Modulus of a family of rectifiable arcs}

There is a natural way to lift the notion of Modulus for a family of Radon measures in $\mathcal{M}_{+}(X)$ to a corresponding Modulus for a collection of rectifiable arcs: it is sufficient to assign a map $M$ : $\mathrm{RA}(X) \rightarrow \mathcal{M}_{+}(X)$ and for every $\Gamma \subset \mathrm{RA}(X)$ set $\operatorname{Mod}_{p, M}(\Gamma):=\operatorname{Mod}_{p}(M(\Gamma))$. Clearly such a notion depends on the choice of $M$; in these notes we will consider two (slightly) different situations: the first one correspond to the most classic and widely used choice of the $\mathscr{H}^{1}$-measure carried by $\gamma$ of (3.44)

$$
\mathrm{M} \gamma:=\nu_{\gamma}, \quad \nu_{\gamma}:=\ell(\gamma)\left(R_{\gamma}\right)_{\sharp}\left(\mathscr{L}^{1}\llcorner[0,1]) .\right.
$$

In this case we will keep the standard notation of $\operatorname{Mod}_{p}(\Gamma), \operatorname{Mod}_{p, c}(\Gamma)$; e.g. in the case of $\operatorname{Mod}_{p}$ (7.1) reads

$$
\operatorname{Mod}_{p}(\Gamma):=\operatorname{Mod}_{p}(\mathrm{M}(\Gamma))=\inf \left\{\int_{X} f^{p} \mathrm{~d} \mathfrak{m}: f \in \mathcal{L}_{+}^{p}(X, \mathfrak{m}), \int_{\gamma} f \geq 1 \quad \text { for all } \gamma \in \Gamma\right\},
$$

with obvious modification for $\operatorname{Mod}_{p, c}(\Gamma)$. The second choice corresponds to

$$
\tilde{\mathrm{M}} \gamma=\tilde{\nu}_{\gamma}:=\nu_{\gamma}+\delta_{\gamma_{0}}+\delta_{\gamma_{1}}, \quad \int_{X} f \mathrm{~d}\left(\tilde{\nu}_{\gamma}\right)=f\left(\gamma_{0}\right)+f\left(\gamma_{1}\right)+\int_{\gamma} f
$$


where as usual we write the initial and final points of $\gamma$ as $\gamma_{i}=\mathrm{e}_{i}(\gamma)=R_{\gamma}(i), i=0,1$. We will denote by $\widetilde{\operatorname{Mod}}_{p}(\Gamma)$ the corresponding modulus,

$$
\begin{aligned}
& \widetilde{\operatorname{Mod}}_{p}(\Gamma):=\operatorname{Mod}_{p}(\tilde{\mathrm{M}}(\Gamma)) \\
& =\inf \left\{\int_{X} f^{p} \mathrm{dm}: f \in \mathcal{L}_{+}^{p}(X, \mathfrak{m}), f\left(\gamma_{0}\right)+f\left(\gamma_{1}\right)+\int_{\gamma} f \geq 1 \quad \text { for all } \gamma \in \Gamma\right\} .
\end{aligned}
$$

It is clear that

$$
\widetilde{\operatorname{Mod}}_{p}(\Gamma) \leq \operatorname{Mod}_{p}(\Gamma), \quad \widetilde{\operatorname{Mod}}_{p}(\Gamma) \leq 2^{-p} \mathfrak{m}(X) \quad \text { for every } \Gamma \subset \operatorname{RA}(X)
$$

One main difference between $\operatorname{Mod}_{p}$ and $\widetilde{\operatorname{Mod}}_{p}$ is the behaviour on constant arcs: if $\Gamma$ contains a constant arc than it is clear that $\operatorname{Mod}_{p}(\Gamma)=+\infty$, whereas for a collection $\Gamma_{A}=\left\{\gamma_{x}: x \in A\right\}$ of constant arcs parametrized by a Borel set $A \subset X$ we have $\widetilde{\operatorname{Mod}}_{p}\left(\Gamma_{A}\right)=2^{-p} \mathfrak{m}(A)$.

The notions of $\operatorname{Mod}_{p^{-}}$or $\widetilde{\operatorname{Mod}}_{p^{-}}$-negligible set of arcs (and of properties which hold $\operatorname{Mod}_{p^{-}}$or $\widetilde{\operatorname{Mod}}_{p}$-a.e.) follow accordingly from Definition 7.1. Properties (a-e) of Proposition 7.2 have an obvious version for arcs. The only statements that require some care are Proposition 7.2(f) and Lemma 7.3, since compactness in $\mathrm{RA}(X)$ for subsets $\Gamma$ of arcs is not equivalent to compactness for the corresponding subsets $\mathrm{M}(\Gamma), \tilde{\mathrm{M}}(\Gamma)$ in $\mathcal{M}_{+}(X)$.

Concerning the validity of Proposition 7.2(f), it is sufficient to note that for every nonnegative $\phi \in \mathrm{C}_{b}(X)$ the maps

$$
\begin{aligned}
& \gamma \mapsto \int \phi \mathrm{d}(\mathrm{M} \gamma)=\int_{\gamma} \phi \\
& \gamma \mapsto \int \phi \mathrm{d}(\tilde{\mathrm{M}} \gamma)=\phi\left(\gamma_{0}\right)+\phi\left(\gamma_{1}\right)+\int_{\gamma} \phi
\end{aligned}
$$

are lower semicontinuous, thanks to Theorem 3.13, so that the argument of the proof works as well.

Corollary 7.4. If $\mathrm{K}_{n}$ is a nonincreasing sequence of compact sets in $\mathrm{RA}(X)$ we have $\operatorname{Mod}_{p, c}\left(K_{n}\right) \downarrow$ $\operatorname{Mod}_{p, c}\left(\cap_{n} K_{n}\right), \widetilde{\operatorname{Mod}}_{p, c}\left(K_{n}\right) \downarrow \widetilde{\operatorname{Mod}}_{p, c}\left(\cap_{n} K_{n}\right)$.

Concerning the tightness Lemma 7.3 we have:

Lemma 7.5 (Tightness of $\operatorname{Mod}_{p}$ and $\left.\widetilde{\operatorname{Mod}}_{p}\right)$. Let us suppose that $(X, \mathrm{~d})$ is complete.

(a) For every $\varepsilon>0$ there exists $K_{\varepsilon} \subset \mathrm{RA}(X)$ compact such that $\widetilde{\operatorname{Mod}}_{p}\left(\mathrm{RA}(X) \backslash K_{\varepsilon}\right) \leq \varepsilon$.

(b) For every $\eta, \varepsilon>0$ there exists $K_{\varepsilon} \subset \mathrm{RA}(X)$ compact such that $\operatorname{Mod}_{p}\left(\mathrm{RA}_{\eta}(X) \backslash K_{\varepsilon}\right) \leq \varepsilon$ (where $\mathrm{RA}_{\eta}(X)$ has been defined in (3.52)).

Proof. (a) We can repeat verbatim the proof of Lemma 7.3: keeping the same notation, the main point is that the sets

$$
\tilde{\mathrm{M}}^{-1}\left(E_{k}\right):=\left\{\gamma \in \mathrm{RA}(X): 2+\ell(\gamma) \leq k, \quad \tilde{\mathrm{M}} \gamma\left(X \backslash K_{n}\right) \leq \delta_{n} \text { for every } n \geq k\right\}
$$

are compact in $\mathrm{RA}(X)$ by Theorem 3.13(g): in fact, since $\tilde{\mathrm{M}} \gamma=\delta_{\gamma_{0}}+\delta_{\gamma_{1}}+\nu_{\gamma}$, whenever $\delta_{n}<1$ condition (7.15) yields $\gamma_{0}, \gamma_{1} \in K$, so that also the assumption 2. of Theorem 3.13(g) holds. 
(b) In this case the set $\mathrm{E}_{k}:=\mathrm{M}^{-1}\left(E_{k}\right)$ cannot be compact in general, since it contains all the constant curves, so that there is no hope to construct inner compact approximations $K_{\varepsilon}$ such that $\operatorname{Mod}_{p}\left(\operatorname{RA}(X) \backslash K_{\varepsilon}\right) \downarrow 0$. We thus replace $\mathrm{RA}(X)$ with $\mathrm{RA}_{\eta}(X), \eta>0$, and modify the definition of the sets $E_{k}$ by requiring that the support of $\mu$ intersect $K_{k}$; in terms of $\mathrm{E}_{k}=\mathrm{M}^{-1}\left(E_{k}\right)$ this corresponds to

$$
\left.\mathrm{E}_{k}:=\left\{\gamma \in \mathrm{RA}(X): \ell(\gamma) \leq k, \mathrm{e}(\gamma) \cap K_{k} \neq \emptyset, \quad \nu_{\gamma}\left(X \backslash K_{n}\right)\right) \leq \delta_{n} \text { for every } n \geq k\right\},
$$

which are compact in $\mathrm{RA}(X)$ by Theorem $3.13(\mathrm{~g})$.

To evaluate $\operatorname{Mod}_{p}\left(\operatorname{RA}_{\eta}(X) \backslash E_{k}\right)$ we introduce the functions $f_{k}$ as in (7.7) and we observe that if $\gamma \in \mathrm{RA}_{\eta}(X) \backslash E_{k}$ then we have either $\ell(\gamma)>k$ or $\mathrm{e}(\gamma) \subset X \backslash K_{k}$ or $\nu_{\gamma}\left(X \backslash K_{n}\right)>\delta_{n}$ for some $n \geq k$. In either case the integral of the function $f_{k}+\frac{1}{k}$ along $\gamma$ is greater or equal to 1 : we have just to check the case $\mathrm{e}(\gamma) \cap K_{k}=\emptyset$, for which

$$
\int_{\gamma}\left(f_{k}+\frac{1}{k}\right) \geq a_{k} \ell(\gamma) \geq \eta a_{k} \geq 1 \quad \text { if } \quad a_{k} \geq \eta^{-1}
$$

For sufficiently big $k$ we thus obtain the same estimates (7.8) and (7.9).

\subsection{Notes}

The notion of $p$-Modulus has been introduced by Fuglede [31] in the natural framework of collection of positive measures, as in [2]. Its application to the metric theory of Sobolev spaces has been proposed in [47] and further studied in [58], where the definition of Newtonian spaces has been introduced. We refer to [17, 46] for a comprehensive presentation of this topic.

The tightness estimate for $\operatorname{Mod}_{p}$ in $\mathcal{M}_{+}(X)$ has been introduced by [2], where it plays a crucial role. Here we used the same approach to derive tightness estimates directly in $\mathrm{RA}(X)$, for the two relevant embeddings of $\mathrm{RA}(X)$ in $\mathcal{M}_{+}(X)$ giving raise to $\operatorname{Mod}_{p}$ and $\widetilde{\operatorname{Mod}}_{p}$.

\section{8 (Nonparametric) Dynamic plans with barycenter in $L^{q}(X, \mathfrak{m})$}

Let us keep the main Assumption of page 70. We denote by $q=p^{\prime}=p /(p-1) \in(1, \infty)$ the conjugate exponent of $p$.

Definition 8.1 ((Nonparametric) dynamic plans). A (nonparametric) dynamic plan is a Radon measure $\boldsymbol{\pi} \in \mathcal{M}_{+}(\mathrm{RA}(X))$ on $\mathrm{RA}(X)$ such that

$$
\boldsymbol{\pi}(\ell):=\int_{\operatorname{RA}(X)} \ell(\gamma) \mathrm{d} \boldsymbol{\pi}(\gamma)<\infty
$$

Since $\mathrm{RA}(X)$ is a $F_{\sigma}$-subset of $\mathrm{A}(X)$, a dynamic plan can also be considered as the restriction of a Radon measure $\boldsymbol{\pi}^{\prime}$ on $\mathrm{A}(X)$ satisfying $\int \ell \mathrm{d} \boldsymbol{\pi}^{\prime}<\infty$; in particular $\boldsymbol{\pi}^{\prime}$ is concentrated on $\operatorname{RA}(X)$, i.e. $\ell(\gamma)<\infty$ for $\pi^{\prime}$-a.e. $\gamma$. Using the universally Lusin-measurable map $G: \operatorname{RA}(X) \rightarrow$ $\mathrm{BVC}_{c}([0,1] ; X)(3.41)$ we can also lift $\boldsymbol{\pi}$ to a Radon measure $\tilde{\boldsymbol{\pi}}=R_{\sharp} \boldsymbol{\pi}$ on $\mathrm{C}([0,1] ; X)$ concentrated on the set $\mathrm{BVC}_{c}([0,1] ; X)$ (3.37). Conversely, any Radon measure $\tilde{\boldsymbol{\pi}}$ on $\mathrm{C}([0,1] ; X)$ concentrated on $\operatorname{BVC}([0,1] ; X)$ yields the Radon measure $\boldsymbol{\pi}:=\mathfrak{q}_{\sharp} \tilde{\boldsymbol{\pi}}$ on $\mathrm{RA}(X)$. Notice that $\mathfrak{q}_{\sharp}\left(R_{\sharp} \boldsymbol{\pi}\right)=\boldsymbol{\pi}$.

If $\boldsymbol{\pi}$ is a dynamic plan in $\mathcal{M}_{+}(\operatorname{RA}(X))$, thanks to Theorem 3.13(e) and Fubini's Theorem [25, Chap. II-14], we can define the Borel measure $\mu_{\boldsymbol{\pi}}:=\operatorname{Proj}(\boldsymbol{\pi}) \in \mathcal{M}_{+}(X)$ by the formula

$$
\int f \mathrm{~d} \mu_{\boldsymbol{\pi}}:=\iint_{\gamma} f \mathrm{~d} \boldsymbol{\pi}(\gamma) \text { for every bounded Borel function } f: X \rightarrow \mathbb{R} \text {. }
$$


It is not difficult to show that $\mu_{\boldsymbol{\pi}}$ is a Radon measure with total mass $\pi(\ell)$ given by (8.1): in fact, setting $\operatorname{RA}^{L}(X):=\{\gamma \in \operatorname{RA}(X): \ell(\gamma) \leq L\}$, for every $\varepsilon>0$ we can find a length $L>0$ such that $\boldsymbol{\pi}\left(\mathrm{RA}(X) \backslash \mathrm{RA}^{L}(X)\right) \leq \varepsilon / 2$. Since $\pi$ is Radon and $R$ is Lusin $\pi$-measurable, we can also find a compact set $\mathscr{K}_{\varepsilon} \subset \mathrm{RA}^{L}(X)$ on which $R$ is continuous and $\boldsymbol{\pi}\left(\mathrm{RA}(X) \backslash \mathscr{K}_{\varepsilon}\right) \leq \varepsilon /(2 L)$. We deduce that $\mu_{\boldsymbol{\pi}}$ is $\varepsilon$-concentrated on the compact $K_{\varepsilon}:=\left\{R_{\gamma}(t): t \in[0,1], \gamma \in \mathscr{K}_{\varepsilon}\right\}=\mathrm{e}\left([0,1] \times R\left(\mathscr{K}_{\varepsilon}\right)\right)$, i.e. $\mu_{\boldsymbol{\pi}}\left(X \backslash K_{\varepsilon}\right) \leq \varepsilon$, since

$$
\begin{aligned}
\mu_{\boldsymbol{\pi}}\left(X \backslash K_{\varepsilon}\right) & =\int\left(\int_{\gamma} \chi_{X \backslash K_{\varepsilon}}\right) \mathrm{d} \boldsymbol{\pi}(\gamma)=\int\left(\ell(\gamma) \int_{0}^{1} \chi_{X \backslash K_{\varepsilon}}\left(R_{\gamma}(t)\right) \mathrm{d} t\right) \mathrm{d} \boldsymbol{\pi}(\gamma) \\
& \leq L \int\left(\int_{0}^{1} \chi_{X \backslash K_{\varepsilon}}\left(R_{\gamma}(t)\right) \mathrm{d} t\right) \mathrm{d} \boldsymbol{\pi}(\gamma)=L\left(\mathscr{L}^{1} \otimes \boldsymbol{\pi}\right)\left\{(t, \gamma): R_{\gamma}(t) \notin K_{\varepsilon}\right\} \\
& \leq L \boldsymbol{\pi}\left(\left\{\gamma \in \operatorname{RA}(X): \gamma \notin \mathscr{K}_{\varepsilon}\right\}\right) \leq \varepsilon / 2 .
\end{aligned}
$$

Notice that $\mu_{\boldsymbol{\pi}}$ can be considered as the integral w.r.t. $\pi$ of the Borel family of measures $\nu_{\gamma}, \gamma \in$ $\mathrm{RA}(X)$ [25, Chap. II-13], in the sense that

$$
\int_{X} f \mathrm{~d} \mu_{\boldsymbol{\pi}}(x)=\int_{\operatorname{RA}(X)}\left(\int_{X} f \mathrm{~d} \nu_{\gamma}\right) \mathrm{d} \boldsymbol{\pi}(\gamma) .
$$

Definition 8.2. We say that $\pi \in \mathcal{M}_{+}(\mathrm{RA}(X))$ has barycenter in $L^{q}(X, \mathfrak{m})$ if there exists $h \in$ $L^{q}(X, \mathfrak{m})$ such that $\mu_{\boldsymbol{\pi}}=h \mathfrak{m}$, or, equivalently, if

$$
\iint_{\gamma} f \mathrm{~d} \boldsymbol{\pi}(\gamma)=\int f h \mathrm{~d} \mathfrak{m} \text { for every } f \in \mathrm{B}_{b}(X)
$$

and we call $\operatorname{Bar}_{q}(\boldsymbol{\pi}):=\|h\|_{L^{q}(X, \mathfrak{m})}$ the barycentric q-entropy of $\boldsymbol{\pi}$. We will denote by $\mathcal{B}_{q}$ the set of all plans with barycenter in $L^{q}(X, \mathfrak{m})$ and we will set $\operatorname{Bar}_{q}(\boldsymbol{\pi}):=+\infty$ if $\boldsymbol{\pi} \notin \mathcal{B}_{q}$.

$\operatorname{Bar}_{q}: \mathcal{M}_{+}(\operatorname{RA}(X)) \rightarrow[0,+\infty]$ is a convex and positively 1 -homogeneous functional. When $q=1 \boldsymbol{\pi}$ has barycenter in $L^{1}(X, \mathfrak{m})$ if and only if $\mu_{\boldsymbol{\pi}} \ll \mathfrak{m}$ and in this case $\operatorname{Bar}_{1}(\boldsymbol{\pi})=\boldsymbol{\pi}(\ell)=$ $\int \ell \mathrm{d} \pi$.

If $q>1$ (which corresponds to our setting, when $q$ is the dual of $p$ ) then $\operatorname{Bar}_{q}$ is also lower semicontinuous w.r.t. the weak topology of $\mathcal{M}_{+}(\operatorname{RA}(X))$, a property which can be easily deduced by the equivalent representation formula (8.7) below. Notice that $\operatorname{Bar}_{q}(\boldsymbol{\pi})=0$ iff $\boldsymbol{\pi}$ is concentrated on the set of constant arcs in $\mathrm{RA}(X)$.

$\operatorname{Bar}_{q}(\boldsymbol{\pi})$ has two equivalent representation. The first one is related to the $L^{q}$ entropy of the projected measure $\mu_{\boldsymbol{\pi}}=\operatorname{Proj}(\boldsymbol{\pi})$ with respect to $\mathfrak{m}$ :

$$
\frac{1}{q} \operatorname{Bar}_{q}^{q}(\boldsymbol{\pi})=\mathscr{L}_{q}\left(\mu_{\boldsymbol{\pi}} \mid \mathfrak{m}\right)
$$

where for an arbitrary $\mu \in \mathcal{M}_{+}(X)$

$$
\mathscr{L}_{q}(\mu \mid \mathfrak{m}):= \begin{cases}\frac{1}{q} \int_{X}\left(\frac{\mathrm{d} \mu}{\mathrm{d} \mathfrak{m}}\right)^{q} \mathrm{~d} \mathfrak{m} & \text { if } \mu \ll \mathfrak{m}, \\ +\infty & \text { otherwise }\end{cases}
$$

A second interpretation arises from the dual characterization of $\mathscr{L}_{q}$, since $q=p^{\prime} \in(1,+\infty)[49$, Thm. 2.7, Rem. 2.8]

$$
\mathscr{L}_{q}(\mu \mid \mathfrak{m})=\sup \left\{\int_{X} f \mathrm{~d} \mu-\frac{1}{p} \int_{X} f^{p} \mathrm{~d} \mathfrak{m}: f \in \mathrm{C}_{b}(X), \quad f \geq 0\right\} .
$$


We immediately obtain

$$
\frac{1}{q} \operatorname{Bar}_{q}^{q}(\boldsymbol{\pi})=\sup \left\{\iint_{\gamma} f \mathrm{~d} \boldsymbol{\pi}(\gamma)-\frac{1}{p} \int_{X} f^{p} \mathrm{~d} \mathfrak{m}: f \in \mathrm{C}_{b}(X), \quad f \geq 0\right\} .
$$

Similarly (see Lemma A.7 in the Appendix) we can easily check that

Lemma 8.3. If $q \in(1, \infty), p=q^{\prime}$, a Radon measure $\pi$ on $\operatorname{RA}(X)$ has barycenter in $L^{q}(X, \mathfrak{m})$ if there exists $c \in[0, \infty)$ such that

$$
\int_{\operatorname{RA}(X)} \int_{\gamma} f \mathrm{~d} \boldsymbol{\pi}(\gamma) \leq c\|f\|_{L^{p}(X, \mathfrak{m})} \quad \text { for every } f \in \mathcal{L}_{+}^{p}(X, \mathfrak{m}) .
$$

In this case $\operatorname{Bar}_{q}(\boldsymbol{\pi})$ is the minimal constant $c$ in (8.9). Moreover, it is equivalent to check (8.9) on nonnegative functions $f \in \mathrm{C}_{b}(X)$.

Definition 8.4 ( $\mathcal{B}_{q}$-negligible sets). We say that a set $\Gamma \subset \mathrm{RA}(X)$ is $\mathcal{B}_{q}$-negligible if $\pi(\Gamma)=0$ for every $\boldsymbol{\pi} \in \mathcal{B}_{q}$. Similarly, a property $P$ on the set of arcs $\operatorname{RA}(X)$ holds $\mathcal{B}_{q}$-a.e. if $\{\gamma \in \operatorname{RA}(X)$ : $P(\gamma)$ does not hold $\}$ is contained in a $\mathcal{B}_{q}$-negligible set.

It is easy to check that for every Borel set $B \subset X$ with $\mathfrak{m}(B)=0$ the set

$$
\left\{\gamma \in \operatorname{RA}(X): \nu_{\gamma}(B)>0\right\} \quad \text { is } \mathcal{B}_{q} \text {-negligible. }
$$

There is a simple duality inequality, involving the minimization in the definition (7.1) of $\operatorname{Mod}_{p}$ and a maximization among all $\boldsymbol{\pi}$ 's with barycenter in $L^{q}(X, \mathfrak{m})$. To see it, let's take $f \in \mathcal{L}_{+}^{p}(X, \mathfrak{m})$ such that $\int_{\gamma} f \geq 1$ on $\Gamma \subset \mathcal{M}_{+}(X)$. Then, if $\Gamma$ is universally Lusin measurable we may take any plan $\pi$ with barycenter in $L^{q}(X, \mathfrak{m})$ to obtain

$$
\boldsymbol{\pi}(\Gamma) \leq \iint_{\gamma} f \mathrm{~d} \boldsymbol{\pi}(\gamma) \leq \operatorname{Bar}_{q}(\boldsymbol{\pi})\|f\|_{p} \quad \text { if } \int_{\gamma} f \geq 1 \text { for every } \gamma \in \Gamma .
$$

By the definition of $\operatorname{Mod}_{p}$ we obtain

$$
\boldsymbol{\pi}(\Gamma) \leq \operatorname{Bar}_{q}(\boldsymbol{\pi}) \operatorname{Mod}_{p}^{1 / p}(\Gamma)
$$

In particular we have

$$
\operatorname{Mod}_{p}(\Gamma)=0 \quad \Longrightarrow \quad \pi(\Gamma)=0 \quad \text { for all } \boldsymbol{\pi} \in \mathcal{B}_{q}
$$

Lemma 8.5 (An equi-tightness criterium). Let us suppose that $(X, \mathrm{~d})$ is complete and let $\mathcal{K}$ be a subset of $\mathcal{B}_{q}$ satisfying the following conditions:

(T1) There exist constants $C_{1}, C_{2}>0$ such that

$$
\boldsymbol{\pi}(\mathrm{RA}(X)) \leq C_{1}, \quad \operatorname{Bar}_{q}(\boldsymbol{\pi}) \leq C_{2} \quad \text { for every } \boldsymbol{\pi} \in \mathcal{K} .
$$

(T2) For every $\varepsilon>0$ there exists a $\tau$-compact set $H_{\varepsilon} \subset X$ such that

$$
\boldsymbol{\pi}\left(\left\{\gamma \in \operatorname{RA}(X): \mathrm{e}(\gamma) \cap H_{\varepsilon}=\emptyset\right\}\right) \leq \varepsilon \quad \text { for every } \boldsymbol{\pi} \in \mathcal{K}
$$


Then $\mathcal{K}$ is relatively compact in $\mathcal{M}_{+}(\mathrm{RA}(X))$.

Proof. We want to apply Prokhorov's Theorem 2.2 so that for every $\varepsilon>0$ we have to exhibit a compact set $K_{\varepsilon} \subset \mathrm{RA}(X)$ such that $\pi\left(\mathrm{RA}(X) \backslash K_{\varepsilon}\right) \leq \varepsilon$.

Let $K_{n}, m_{n}, a_{n}, \delta_{n}, f_{k}$ be defined as in the proof of Lemma 7.3 and let us set $\mathrm{E}_{k, \xi}:=\mathrm{F}_{k} \cap \mathrm{G}_{\xi}$ where

$$
\begin{aligned}
& \left.\mathrm{F}_{k}:=\left\{\gamma \in \operatorname{RA}(X): \ell(\gamma) \leq k, \nu_{\gamma}\left(X \backslash K_{n}\right)\right) \leq \delta_{n} \text { for every } n \geq k\right\}, \\
& \mathrm{G}_{\xi}:=\left\{\gamma \in \operatorname{RA}(X): \mathrm{e}(\gamma) \cap H_{\xi} \neq \emptyset\right\} .
\end{aligned}
$$

For every $k \in \mathbb{N}$ and $\xi>0 \mathrm{E}_{k, \xi}$ are compact by Theorem 3.13(g).

Let us estimate $\boldsymbol{\pi}\left(\mathrm{RA}(X) \backslash \mathrm{E}_{k, \xi}\right)$ for $\boldsymbol{\pi} \in \mathcal{K}$. By (T2) we know that $\boldsymbol{\pi}\left(\mathrm{RA}(X) \backslash \mathrm{G}_{\xi}\right) \leq \xi$. On the other hand, since $\int_{\gamma}\left(f_{k}+1 / k\right) \geq 1$ for every $\gamma \in \mathrm{RA}(X) \backslash \mathrm{F}_{k}$ we have

$$
\boldsymbol{\pi}\left(\mathrm{RA}(X) \backslash \mathrm{F}_{k}\right) \stackrel{(8.11)}{\leq} C_{2}\left\|f_{k}+1 / k\right\|_{L^{p}(X, \mathfrak{m})} \stackrel{(7.8)}{\leq} C_{2} M_{k},
$$

where $M_{k}:=\left(m_{k}\right)^{1 /(2 p)}+(\mathfrak{m}(X))^{1 / p} / k \downarrow 0$ as $k \rightarrow \infty$. We deduce $\boldsymbol{\pi}\left(\mathrm{RA}(X) \backslash \mathrm{E}_{k, \xi}\right) \leq \xi+C_{2} M_{k}$ and the thesis follows by choosing $K_{\varepsilon}:=\mathrm{E}_{k, \xi}$ for $\xi+C_{2} M_{k}<\varepsilon$.

It is easy to check that (T2) is also a necessary condition for the equi-tightness of $\mathcal{K}$. In fact, if $\mathcal{K}$ is equi-tight in $\mathcal{M}_{+}(\mathrm{RA}(X))$ then the collection $\mathcal{K}^{\prime}:=\left\{\left(\mathrm{e}_{0}\right)_{\sharp} \boldsymbol{\pi}: \boldsymbol{\pi} \in \mathcal{K}\right\}$ is equi-tight in $\mathcal{M}_{+}(X)$ (since $\mathrm{e}_{0}$ is a continuous map from $\mathrm{RA}(X)$ to $X$ ). Therefore, for every $\varepsilon>0$ there exists a compact set $K_{\varepsilon} \subset X$ such that $\boldsymbol{\pi}\left(\left\{\gamma \in \mathrm{RA}(X): \gamma_{0} \notin K_{\varepsilon}\right\}\right) \leq \varepsilon$, which clearly yields (8.15).

It is interesting to notice that if $\mathcal{K} \subset \mathcal{M}_{+}(\mathrm{RA}(X))$ satisfies the property (T1) above and

$$
\ell(\gamma) \geq C_{3}>0 \quad \pi \text {-a.e. for every } \boldsymbol{\pi} \in \mathcal{K}
$$

then (T2) is satisfied as well. In fact, if $K_{n} \subset X$ is a compact set with $\mathfrak{m}\left(X \backslash K_{n}\right) \leq m_{n}$ and $\mathrm{G}_{n}:=\left\{\gamma \in \mathrm{RA}(X): \mathrm{e}(\gamma) \cap K_{n}=\emptyset\right\}$ for every $\boldsymbol{\pi} \in \mathcal{K}$ we have

$$
\begin{aligned}
\boldsymbol{\pi}\left(\mathrm{G}_{n}\right) & \leq \frac{1}{C_{3}} \int_{\mathrm{G}_{n}} \ell(\gamma) \mathrm{d} \boldsymbol{\pi}(\gamma) \leq \frac{1}{C_{3}} \int\left(\int_{\gamma} \chi_{X \backslash K_{n}}\right) \mathrm{d} \boldsymbol{\pi}(\gamma) \\
& =\frac{1}{C_{3}} \mu_{\boldsymbol{\pi}}\left(X \backslash K_{n}\right) \leq \frac{1}{C_{3}} \operatorname{Bar}_{q}(\boldsymbol{\pi}) m_{n}^{1 / p} \leq \frac{C_{2}}{C_{3}} m_{n}^{1 / p} \downarrow 0 \quad \text { as } n \rightarrow \infty .
\end{aligned}
$$

The inequality (8.11) motivates the next definition.

Definition 8.6 ( $p$-content). If $\Gamma \subset \mathrm{RA}(X)$ is a universally measurable set we say that $\Gamma$ has finite content if there exists a constant $c \geq 0$ such that

$$
\boldsymbol{\pi}(\Gamma) \leq c \operatorname{Bar}_{q}(\boldsymbol{\pi}) \quad \text { for every } \boldsymbol{\pi} \in \mathcal{M}_{+}(\mathrm{RA}(X)) .
$$

In this case, the p-content of $\Gamma \operatorname{Cont}_{p}(\Gamma)$ is the minimal constant $c$ satisfying (8.18). If $\Gamma$ has not finite content we set $\operatorname{Cont}_{p}(\Gamma):=+\infty$.

Notice that if $\Gamma$ contains a constant arc we get $\operatorname{Cont}_{p}(\Gamma)=+\infty$; conversely, $\operatorname{Cont}_{p}(\Gamma)=0$ if and only if $\Gamma$ is $\mathcal{B}_{q}$-negligible. We can formulate (8.18) in the equivalent form

$$
\operatorname{Cont}_{p}(\Gamma)=\sup _{\boldsymbol{\pi} \in \mathcal{M}_{+}(\operatorname{RA}(X)), \operatorname{Bar}_{q}(\boldsymbol{\pi})>0} \frac{\boldsymbol{\pi}(\Gamma)}{\operatorname{Bar}_{q}(\boldsymbol{\pi})},
$$


and we can also limit the sup in (8.19) and the condition (8.18) to probability plans (i.e. $\pi(\operatorname{RA}(X))=$ 1) concentrated on $\Gamma$.

By Lemma A.7 we easily find the equivalent characterizations of Cont ${ }_{p}$ :

$$
\frac{1}{p} \operatorname{Cont}_{p}^{p}(\Gamma)=\sup _{\boldsymbol{\pi} \in \mathcal{M}_{+}(\operatorname{RA}(X))} \boldsymbol{\pi}(\Gamma)-\frac{1}{q} \operatorname{Bar}_{q}^{q}(\boldsymbol{\pi})
$$

showing that $\frac{1}{p} \operatorname{Cont}_{p}^{p}$ is in fact the Legendre transform of $\frac{1}{q} \operatorname{Bar}_{q}^{q}$.

Let us now address the question of existence of an optimal dynamic plan attaining the supremum in (8.19) (or, equivalently, in (8.20)). The next result corresponds to [2, Lemma 4.4], where however the condition concerning the closure of $\Gamma$ is missing. See also the comments in the Notes 8.1 at the end of this section.

Lemma 8.7. Let us suppose that $(X, \mathrm{~d})$ is complete and let $\Gamma \subset \mathrm{RA}(X)$ be a closed set such that $0<\operatorname{Cont}_{p}(\Gamma)<+\infty$. If there exists a compact set $K \subset X$ such that $\mathrm{e}(\gamma) \cap K \neq \emptyset$ for every $\gamma \in \Gamma$ (in particular if $\Gamma$ is compact), then there exists an optimal plan $\pi_{\Gamma}$ with barycenter in $L^{q}(X, \mathfrak{m})$ attaining the supremum in (8.20). $\pi_{\Gamma}$ is concentrated on $\Gamma$ and satisfies

$$
\boldsymbol{\pi}_{\Gamma}(\Gamma)=\operatorname{Cont}_{p}^{p}(\Gamma)=\operatorname{Bar}_{q}^{q}\left(\boldsymbol{\pi}_{\Gamma}\right) .
$$

In particular, $\tilde{\boldsymbol{\pi}}_{\Gamma}:=\left(\boldsymbol{\pi}_{\Gamma}(\Gamma)\right)^{-1} \boldsymbol{\pi}_{\Gamma}$ is a probability plan and

$$
\operatorname{Cont}_{p}(\Gamma) \operatorname{Bar}_{q}\left(\tilde{\boldsymbol{\pi}}_{\Gamma}\right)=1 .
$$

Proof. Taking perturbations of the form $\pi \rightarrow \kappa \pi, \kappa>0$, we immediately see that we can restrict the maximization to plans satisfying

$$
\boldsymbol{\pi}(\Gamma)=\boldsymbol{\pi}(\mathrm{RA}(X))=\operatorname{Bar}_{q}^{q}(\boldsymbol{\pi}) \leq \operatorname{Cont}_{p}^{p}(\Gamma) .
$$

It is also easy to see that it is possible to restrict the maximization to plans concentrated on $\Gamma$, since the restriction $\boldsymbol{\pi} \mapsto \overline{\boldsymbol{\pi}}=\chi_{\Gamma} \boldsymbol{\pi}$ satisfies $\overline{\boldsymbol{\pi}}(\Gamma)=\boldsymbol{\pi}(\Gamma)$ and $\operatorname{Bar}_{q}(\overline{\boldsymbol{\pi}}) \leq \operatorname{Bar}_{q}(\boldsymbol{\pi})$. Since $\Gamma$ is closed the functional of (8.20) is upper semicontinuous and therefore it admits a maximum on the compact set defined by (8.22).

\subsection{Notes}

The notions of barycentric entropy and content have been introduced in [2] for measures in $\mathcal{M}_{+}(X)$, in order to provide an equivalent measure-theoretic characterization of the $\operatorname{modulus} \operatorname{Mod}_{p}$. Here we decided to focus mainly on nonparametric dynamic plans and to develop the main properties in the more restrictive setting characterized by the embedding $\mathrm{M}: \mathrm{RA}(X) \rightarrow \mathcal{M}_{+}(X)$ of 7.10 , which is well adapted to the classic modulus $\operatorname{Mod}_{p}$ on arcs. In 10.2 we will also briefly discuss the notions of barycentric entropy and content related to $\widetilde{\operatorname{Mod}}_{p}$ and to the embedding $\tilde{\mathrm{M}}$ of (7.12).

The equi-tightness criterium 8.5 requires slightly more restrictive assumptions than in [12] since here compactness is obtained directly in $\mathcal{M}_{+}(\mathrm{RA}(X))$ instead of $\mathcal{M}_{+}\left(\mathcal{M}_{+}(X)\right)$. Notice that the class of constant arcs is homeomorphic to $X$ in $\mathrm{RA}(X)$, whereas it is identified with the null measure in $\mathcal{M}_{+}(X)$.

The existence of an optimal plan attaining (8.21) requires at least the closure of $\Gamma$ : this condition should also be added to Lemma 4.4, Corollary 5.2(b) and Theorem 7.2 of [12]. Notice however that the main consequences [2, Theorem 8.3, Corollary 8.7] of Theorem 7.2 in [2] still hold, since they only require the existence of a nontrivial dynamic plan in $\mathcal{B}_{q}$ giving positive mass to $\Gamma$ whenever $\operatorname{Mod}_{p}(\Gamma)>0$ : thanks to Choquet theorem this property holds for an arbitrary Souslin set $\Gamma$ and does not require its closedness. We will also discuss these aspects in the next Section 9, see Theorem 9.2. 


\section{Equivalence between $\operatorname{Cont}_{p}$ and $\operatorname{Mod}_{p}$}

In this Section we always refer to the main Assumption of page 70. We have seen that $\operatorname{Cont}_{p}, \operatorname{Mod}_{p}, \operatorname{Mod}_{p, c}$ satisfy the property

$$
\operatorname{Cont}_{p}^{p} \leq \operatorname{Mod}_{p} \leq \operatorname{Mod}_{p, c} \quad \text { on universally measurable subsets of } \operatorname{RA}(X) .
$$

We first prove that $(9.1)$ is in fact an identity if $\Gamma$ is compact.

Theorem 9.1. If $\Gamma$ is a compact subset of $\mathrm{RA}(X)$ we have

$$
\operatorname{Cont}_{p}^{p}(\Gamma)=\operatorname{Mod}_{p}(\Gamma)=\operatorname{Mod}_{p, c}(\Gamma) .
$$

Proof. We will set $\mathcal{M}_{+}(\Gamma):=\left\{\boldsymbol{\pi} \in \mathcal{M}_{+}(\mathrm{RA}(X)): \operatorname{supp}(\boldsymbol{\pi}) \subset \Gamma\right\}$. Since $\ell$ is a lower semicontinuous map, the minimum $\ell_{0}:=\min _{\gamma \in \Gamma} \ell(\gamma)$ is attained. If $\ell_{0}=0 \Gamma$ contains a constant arc and (9.2) is trivially satisfied since the common value is $+\infty$. We can thus assume $\ell_{0}>0$.

We will prove (9.2) by using a minimax argument by applying Von Neumann Theorem A.8.

First of all we observe that for every $f \in \mathrm{C}_{b}(X)$

$$
\int_{\gamma} f \geq 1 \text { for every } \gamma \in \Gamma \quad \Leftrightarrow \quad \sup \left\{\int\left(1-\int_{\gamma} f\right) \mathrm{d} \boldsymbol{\pi}(\gamma): \boldsymbol{\pi} \in \mathcal{M}_{+}(\Gamma)\right\}=0,
$$

so that

$$
\begin{gathered}
\frac{1}{p} \operatorname{Mod}_{p, c}(\Gamma)=\inf _{f \in \mathrm{C}_{b}(X)} \sup _{\boldsymbol{\pi} \in \mathcal{M}_{+}(\Gamma)} \mathcal{L}(\boldsymbol{\pi}, f), \\
\mathcal{L}(\boldsymbol{\pi}, f):=\frac{1}{p} \int_{X} f^{p} \mathrm{~d} \mathfrak{m}+\int\left(1-\int_{\gamma} f\right) \mathrm{d} \boldsymbol{\pi}(\gamma) .
\end{gathered}
$$

By choosing $f_{\star} \equiv k \geq 2 / \ell_{0}$ we clearly have

$$
\mathcal{L}\left(\boldsymbol{\pi}, f_{*}\right)=\frac{1}{p} \int_{X} f^{p} \mathrm{~d} \mathfrak{m}+\int\left(1-\int_{\gamma} f\right) \mathrm{d} \boldsymbol{\pi}(\gamma) \leq c_{k}-\boldsymbol{\pi}(\Gamma), \quad c_{k}:=\frac{1}{p} \mathfrak{m}(X) k^{p},
$$

so that choosing $D_{\star}<\frac{1}{p} \operatorname{Mod}_{p, c}(\Gamma)$ and $c_{k}$ sufficiently big, the set $\left\{\boldsymbol{\pi} \in \mathcal{M}_{+}(\Gamma): \mathcal{L}\left(\boldsymbol{\pi}, f_{*}\right) \geq D_{*}\right\}$ is not empty (it contains the null plan) and it is contained in the compact set $\left\{\boldsymbol{\pi} \in \mathcal{M}_{+}(\Gamma): \boldsymbol{\pi}(\Gamma) \leq\right.$ $\left.c_{k}-D_{*}\right\}$. Condition (A.12) is thus satisfied and we deduce

$$
\begin{aligned}
\frac{1}{p} \operatorname{Mod}_{p, c}(\Gamma) & =\max _{\boldsymbol{\pi} \in \mathcal{M}_{+}(\Gamma)} \inf _{f \in \mathrm{C}_{b}(X)} \mathcal{L}(\boldsymbol{\pi}, f) \\
& =\max _{\boldsymbol{\pi} \in \mathcal{M}_{+}(\Gamma)} \boldsymbol{\pi}(\Gamma)-\sup \iint_{\gamma} f \mathrm{~d} \boldsymbol{\pi}(\gamma)-\frac{1}{p} \int_{X} f^{p} \mathrm{~d} \mathfrak{m} \\
& =\max _{\boldsymbol{\pi} \in \mathcal{M}_{+}(\Gamma)} \boldsymbol{\pi}(\Gamma)-\frac{1}{q} \operatorname{Bar}_{q}^{q}(\boldsymbol{\pi}) \stackrel{(8.20)}{=} \operatorname{Cont}_{p}^{p}(\Gamma) .
\end{aligned}
$$

Theorem 9.1 has an important implication in terms of Choquet capacity; we refer to [25, Chap. III, $\S 2]$ and to the brief account given in Section A.4 of the Appendix. Recall that $\mathscr{B}(Y)$ (resp. $\mathscr{K}(Y)$ ) will denote the collection of all the Borel (resp. compact) subsets of a Hausdorff space $Y$.The definition and the main properties of Souslin and Analytic sets are briefly recalled in $\S$ A.3.

Theorem 9.2. Let $\mathbb{X}=(X, \tau, \mathrm{d}, \mathfrak{m})$ be an e.m.t.m. space. 
(a) $\operatorname{Mod}_{p}$ is a Choquet $\mathscr{K}\left(\mathrm{RA}(X), \tau_{\mathrm{A}}\right)$-capacity in $\mathrm{RA}(X)$.

(b) For every universally measurable $\Gamma \subset \mathrm{RA}(X)$

$$
\operatorname{Cont}_{p}(\Gamma)=\sup \left\{\operatorname{Cont}_{p}(\mathrm{~K}): \mathrm{K} \subset \Gamma, \mathrm{K} \text { compact }\right\} .
$$

(c) If $(X, \mathrm{~d})$ is complete and $(X, \tau)$ is Souslin, then every $\mathscr{B}\left(\mathrm{RA}(X), \tau_{\mathrm{A}}\right)$-analytic set $\Gamma$ (in particular, every Souslin set) is $\operatorname{Mod}_{p}$-capacitable

(d) If $(X, \mathrm{~d})$ is complete and $(X, \tau)$ is Souslin, then every $\mathscr{B}\left(\mathrm{RA}(X), \tau_{\mathrm{A}}\right)$-analytic set $\Gamma$ satisfies $\operatorname{Mod}_{p}(\Gamma)=\operatorname{Cont}_{p}^{p}(\Gamma)$. In particular $\Gamma$ is $\operatorname{Mod}_{p}$-negligible if and only if it is $\mathcal{B}_{q}$-negligible.

Proof. (a) Proposition 7.2(e,f) and the fact that $\operatorname{Mod}_{p, c}=\operatorname{Mod}_{p}$ if the set is compact by Theorem 9.1 give us that $\operatorname{Mod}_{p}$ is a $\mathscr{K}$-capacity in $\left(\mathrm{RA}(X), \tau_{\mathrm{A}}\right)$.

(b) By (8.20) for every $S<\operatorname{Cont}_{p}(\Gamma)$ we can find $\pi \in \mathcal{B}_{q}$ such that

$$
\frac{1}{p} S^{p}<\boldsymbol{\pi}(\Gamma)-\frac{1}{q} \operatorname{Bar}_{q}(\boldsymbol{\pi})
$$

Since $\Gamma$ is $\pi$-measurable and $\pi$ is Radon, we can find a compact set $K \subset \Gamma$ such that

$$
\frac{1}{p} S^{p}<\boldsymbol{\pi}(\mathrm{K})-\frac{1}{q} \operatorname{Bar}_{q}(\boldsymbol{\pi}) \leq \frac{1}{p} \operatorname{Cont}_{p}(\mathrm{~K}),
$$

which eventually yields (9.4) since $S$ is arbitrary.

(c) Let us now assume that $(X, \mathrm{~d})$ is complete and $(X, \tau)$ is Souslin and let us prove that every $\mathscr{B}$ analytic set is capacitable. By Choquet's Theorem A.6, it is sufficient to prove that every Borel set is capacitable. By Corollary 3.7 we know that $\left(\mathrm{RA}(X), \tau_{\mathrm{A}}, \mathrm{d}_{\mathrm{A}}\right)$ admits an auxiliary topology $\tau_{\mathrm{A}}^{\prime}$.

Let $\Gamma$ be a Borel subset of $\operatorname{RA}(X)$. If $\Gamma$ contains a constant arc there is nothing to prove, so that we can assume that $\Gamma \subset \mathrm{RA}_{0}(X)=\{\gamma \in \mathrm{RA}(X): \ell(\gamma)>0\}$. Recalling the definition of the open sets $\mathrm{RA}_{\eta}(X)$ given in Lemma 7.3 and Proposition 7.2(e), we know that $\operatorname{Mod}_{p}(\Gamma)=\lim _{\eta \downarrow} \operatorname{Mod}_{p}\left(\Gamma_{\eta}\right)$, where $\Gamma_{\eta}:=\Gamma \cap \mathrm{RA}_{\eta}(X)$. It is therefore sufficient to prove that every $\Gamma_{\eta}$ is capacitable. Let us fix $\eta>0$; by applying Lemma 7.3 for every $\varepsilon>0$ we can find a compact set $\mathrm{K}_{\varepsilon} \subset \mathrm{RA}(X)$ such that $\operatorname{Mod}_{p}\left(\Gamma_{\eta} \backslash \mathrm{K}_{\varepsilon}\right) \leq \operatorname{Mod}_{p}\left(\operatorname{RA}_{\eta}(X) \backslash \mathrm{K}_{\varepsilon}\right) \leq \varepsilon$. Since $\operatorname{Mod}_{p}$ is subadditive, it remains to prove that $\Gamma_{\eta} \cap \mathrm{K}_{\varepsilon}$ is capacitable. Notice that $\mathrm{K}_{\varepsilon}$ is also compact with respect to the coarser (metrizable and separable) topology $\tau_{\mathrm{A}}^{\prime}$ and the restriction of $\tau_{\mathrm{A}}^{\prime}$ to $\mathrm{K}_{\varepsilon}$ coincides with $\tau_{\mathrm{A}}$, so that $\left(\mathrm{K}_{\varepsilon}, \tau_{\mathrm{A}}\right)$ is a Polish space. Since $\Gamma_{\eta} \cap \mathrm{K}_{\varepsilon}$ is Borel in $\mathrm{K}_{\varepsilon}$, it is also $\mathscr{F}$-analytic and therefore (being $\mathrm{K}_{\varepsilon}$ compact) $\mathscr{K}$-analytic. By claim (a) above we deduce that $\Gamma_{\eta} \cap \mathrm{K}_{\varepsilon}$ is capacitable.

(d) It is an immediate consequence of the previous claims, recalling that every $\mathscr{B}$-analytic set is universally measurable.

\subsection{Notes}

Theorem 9.1 has been proved in [2] by using a different argument based on Hahn-Banach theorem. The proof presented here, based on Von Neumann theorem, shows more clearly that the definitions of $\operatorname{Mod}_{p}$ and of Cont $p$ rely on dual optimization problems, so that their equality is a nice application of a min-max argument.

Theorem 9.2 strongly relies on Choquet's Theorem. It is interesting to note that the possibility to separate distance and topology in e.m.t.m. space expands the range of application and covers the case of general Souslin 
spaces: the use of an auxiliary topology overcomes the difficulty related to the unknown Souslin character of path spaces (see [25, page 46-III]).

Theorem 9.1 and Theorem 9.2 could be directly stated at the level of modulus and contents on $\mathcal{M}_{+}(X)$ instead of $\operatorname{RA}(X)$, see [2]. We will discuss another important case in $\S 10.2$.

\section{Part IV}

\section{Weak upper gradients and identification of Sobolev spaces}

\section{0 (Nonparametric) Weak upper gradients and weak Sobolev spaces}

In this section we introduce a notion of weak upper gradient modeled on $\mathcal{T}_{q}$-test plans, in the usual setting stated at page 70 .

\section{$10.1 \mathcal{T}_{q}$-test plans and $\mathcal{T}_{q}$-weak upper gradients}

Recall that the (stretched) evaluation maps $\hat{\mathrm{e}}_{t}: \operatorname{RA}(X) \rightarrow X$ are defined by $\hat{\mathrm{e}}_{t}(\gamma):=R_{\gamma}(t)$, see (3.53). We also introduce the restriction maps $\operatorname{Restr}_{s}^{t}: \operatorname{RA}(X) \rightarrow \operatorname{RA}(X), 0 \leq s<t \leq 1$, given by

$$
\operatorname{Restr}_{s}^{t}(\gamma):=\mathfrak{q}\left(R_{\gamma}^{s \rightarrow t}\right), \quad R_{\gamma}^{s \rightarrow t}(r):=R_{\gamma}((1-r) s+r t) \quad r \in[0,1],
$$

where $\mathfrak{q}$ is the projection map from $\mathrm{C}([0,1] ; X)$ to $\mathrm{A}(X)$. Restr ${ }_{s}^{t}$ restricts the arc-length parametrization $R_{\gamma}$ of the arc $\gamma$ to the interval $[s, t]$ and then "stretches" it on the whole of $[0,1]$, giving back the equivalent class in $\operatorname{RA}(X)$. Notice that for every $\gamma \in \operatorname{RA}(X)$

$$
\int_{\operatorname{Restr}_{s}^{t}(\gamma)} f=(t-s) \ell(\gamma) \int_{0}^{1} f\left(R_{\gamma}^{s \rightarrow t}(r)\right) \mathrm{d} r=\ell(\gamma) \int_{s}^{t} f\left(R_{\gamma}(r)\right) \mathrm{d} r .
$$

Definition 10.1 (Nonparametric $\mathcal{T}_{q}$-test plans). Let $q=p^{\prime} \in(1, \infty)$. We call $\mathcal{T}_{q}=\mathcal{T}_{q}(\mathbb{X}) \subset$ $\mathcal{M}_{+}(\mathrm{RA}(X))$ the collection of all (nonparametric) dynamic plan $\pi \in \mathcal{M}_{+}(\mathrm{RA}(X))$ such that

$$
\operatorname{Bar}_{q}(\boldsymbol{\pi})<\infty, \quad \mathscr{L}_{q}\left(\left(\mathrm{e}_{i}\right)_{\sharp} \boldsymbol{\pi} \mid \mathfrak{m}\right)<\infty \quad i=0,1 ;
$$

Dynamic plans in $\mathcal{T}_{q}$ will be also called $\mathcal{T}_{q}$-test plans.

We call $\mathcal{T}_{q}^{*}$ the subset of $\mathcal{T}_{q}$ whose plans $\boldsymbol{\pi}$ satisfy the following property: there exists a constant $c>0$ and a compact set $\mathrm{K} \subset \mathrm{RA}(X)$ (depending on $\pi$ ) such that

$\left(\mathrm{e}_{i}\right)_{\sharp} \boldsymbol{\pi} \leq \mathrm{cm}, i=0,1, \quad \ell_{\mathrm{K}_{\mathrm{K}}}$ is bounded, continuous and strictly positive, $\quad \boldsymbol{\pi}(\mathrm{RA}(X) \backslash \mathrm{K})=0$.

We say that $\boldsymbol{\pi}$ is a stretchable $\mathcal{T}_{q}$-test plan if

$$
\operatorname{Bar}_{q}(\boldsymbol{\pi})<\infty, \quad \mathscr{L}_{q}\left(\left(\hat{\mathrm{e}}_{t}\right)_{\sharp} \boldsymbol{\pi} \mid \mathfrak{m}\right)<\infty \quad \text { for every } t \in[0,1] .
$$

Notice that $\boldsymbol{\pi}$ is a stretchable $\mathcal{T}_{q}$-test plan if and only if $\operatorname{Restr}_{s}^{t}(\boldsymbol{\pi}) \in \mathcal{T}_{q}$ for every $s, t \in[0,1]$, $s<t$. Clearly the class of nonparametric $\mathcal{T}_{q}$-test plans depends on the full e.m.t.m. structure of $\mathbb{X}$; however, when there is no risk of ambiguity, we will simply write $\mathcal{T}_{q}$. 
Definition $10.2\left(\mathcal{T}_{q}\right.$-negligible sets of rectifiable arcs). Let $P$ be a property concerning nonparametric arcs in $\mathrm{RA}(X)$. We say that $P$ holds $\mathcal{T}_{q}$-a.e. (or for $\mathcal{T}_{q}$-almost every arc $\gamma \in \mathrm{RA}(X)$ ) if for any $\pi \in \mathcal{T}_{q}$ the set

$$
N:=\{\gamma: P(\gamma) \text { does not hold }\}
$$

is contained in a $\pi$-negligible Borel set.

Since $\mathcal{T}_{q} \subset \mathcal{B}_{q}$, it is clear that for every Borel set $\Gamma \subset \mathrm{RA}(X)$

$$
\operatorname{Mod}_{p}(\gamma)=0 \Rightarrow \operatorname{Cont}_{p}(\Gamma)=0 \Rightarrow \Gamma \text { is } \mathcal{T}_{q} \text {-negligible }
$$

Notice that we can revert the first implication in (10.6) e.g. when $(X, \tau, \mathrm{d})$ is a Souslin and complete e.m.t. space, see Theorem 9.2.

Lemma 10.3. If a set $N \subset \mathrm{RA}_{0}(X)$ is $\boldsymbol{\pi}$-negligible for every $\boldsymbol{\pi} \in \mathcal{T}_{q}^{*}$ then $N$ is $\mathcal{T}_{q}$-negligible.

Proof. Let us fix $\pi \in \mathcal{T}_{q}$ and let $\pi_{i}=\left(\mathrm{e}_{i}\right)_{\sharp} \pi=h_{i} \mathfrak{m}$ with $h_{i} \in L^{q}(X, \mathfrak{m}), h_{i}$ Borel nonnegative. We set

$$
H_{i, k}:=\left\{x \in X: h_{i}(x) \leq k\right\}, \quad \mathrm{H}_{k}:=\left\{\gamma \in \mathrm{RA}(X): \ell(\gamma) \leq k, \mathrm{e}_{i}(\gamma) \in H_{i, k} i=0,1\right\} .
$$

Clearly

$$
\lim _{k \rightarrow \infty} \boldsymbol{\pi}\left(\mathrm{RA}(X) \backslash \mathrm{H}_{k}\right) \leq \lim _{k \rightarrow \infty}\left(\pi_{0}\left(X \backslash H_{0, k}\right)+\pi_{1}\left(X \backslash H_{1, k}\right)\right)=0 .
$$

We can also find an increasing sequence of compact sets $\mathrm{K}_{n} \subset \mathrm{RA}_{0}(X)$ such that the restriction of $\ell$ to $\mathrm{K}_{n}$ is continuous and strictly positive and $\boldsymbol{\pi}\left(\mathrm{RA}_{0}(X) \backslash \mathrm{K}_{n}\right) \leq 2^{-n}$. It follows that $\boldsymbol{\pi}_{n}:=\left.\boldsymbol{\pi}\right|_{\mathrm{K}_{n} \cap \mathrm{H}_{n}}$ belongs to $\mathcal{T}_{q}^{*}$ and we can find a Borel set $B_{n}$ with $\mathrm{RA}_{0}(X) \supset B_{n} \supset N$ such that $\pi_{n}\left(B_{n}\right)=0$. Setting $B:=\cap_{n} B_{n}$ clearly $B \supset N$ and $\boldsymbol{\pi}_{n}(B)=0$ so that $\boldsymbol{\pi}(B)=0$ as well.

Recall that a Borel function $g: X \rightarrow[0,+\infty]$ is an upper gradient [23] for $f: X \rightarrow \mathbb{R}$ if

$$
\left|\int_{\partial \gamma} f\right| \leq \int_{\gamma} g \text { for every } \gamma \in \operatorname{RA}(X) \quad \text { where } \quad \int_{\partial \gamma} f:=f\left(\gamma_{1}\right)-f\left(\gamma_{0}\right) .
$$

Definition $10.4\left(\mathcal{T}_{q}\right.$-weak upper gradients). Given $f: X \rightarrow \mathbb{R}$, a m-measurable function $g: X \rightarrow$ $[0, \infty]$ is a $\mathcal{T}_{q}$-weak upper gradient (w.u.g.) of $f$ if

$$
\left|\int_{\partial \gamma} f\right| \leq \int_{\gamma} g<\infty \quad \text { for } \mathcal{T}_{q} \text {-almost every } \gamma \in \mathrm{RA}(X)
$$

Remark 10.5 (Truncations). If $T: \mathbb{R} \rightarrow \mathbb{R}$ is a 1-Lipschitz map and $g$ is a $\mathcal{T}_{q}$-w.u.g. of $f$, then $g$ is a $\mathcal{T}_{q}$-w.u.g. of $T \circ f$ as well. Conversely, if $g$ is a $\mathcal{T}_{q}$-w.u.g. of $f_{k}:=-k \vee f \wedge k$ for every $k \in \mathbb{N}$, it is easy to see that $g$ is a $\mathcal{T}_{q}$-w.u.g. of $f$. By this property, in the proof of many statements concerning $\mathcal{T}_{q}$-w.u.g. it will not be restrictive to assume $f$ bounded.

The definition of weak upper gradient enjoys natural invariance properties w.r.t. modifications in $\mathfrak{m}$-negligible sets. We will also show that if $g$ is $\mathfrak{m}$-measurable the integral in (10.8) is well defined for $\mathcal{T}_{q}$-a.e. $\operatorname{arc} \gamma$.

Proposition 10.6 (Measurability and invariance under modifications in $\mathfrak{m}$-negligible sets). 
(a) If $f, \tilde{f}: X \rightarrow \mathbb{R}$ differ in a $\mathfrak{m}$-negligible set then for $\mathcal{T}_{q}$-a.e. arc $\gamma$

$$
f\left(\gamma_{0}\right)=\tilde{f}\left(\gamma_{0}\right), \quad f\left(\gamma_{1}\right)=\tilde{f}\left(\gamma_{1}\right), \quad f \circ R_{\gamma}=\tilde{f} \circ R_{\gamma} \mathscr{L}^{1} \text {-a.e. in }(0,1) .
$$

(b) If $g$ is $\mathfrak{m}$-measurable then the map $s \mapsto g\left(R_{\gamma}(s)\right)$ is $\mathscr{L}^{1}$-measurable for $\mathcal{T}_{q}$-a.e. arc $\gamma$.

(c) Let $f, \tilde{f}: X \rightarrow \mathbb{R}$ and $g, \tilde{g}: X \rightarrow[0, \infty]$ be such that both $\{f \neq \tilde{f}\}$ and $\{g \neq \tilde{g}\}$ are $\mathfrak{m}$-negligible. If $g$ is a $\mathcal{T}_{q}$-weak upper gradient of $f$ then $\tilde{g}$ is a $\mathcal{T}_{q}$-weak upper gradient of $\tilde{f}$.

Proof. (a) Let $N \supset\{f \neq \tilde{f}\}$ be a m-negligible Borel set and let $\pi \in \mathcal{T}_{q}$ be a test plan. We have

$$
\int\left(\int_{\gamma} \chi_{N}\right) \mathrm{d} \boldsymbol{\pi}(\gamma)=\mu_{\boldsymbol{\pi}}(N)=0 \quad \text { since } \mu_{\boldsymbol{\pi}} \ll \mathfrak{m} \text { and } \mathfrak{m}(N)=0,
$$

so that $\int_{\gamma} \chi_{N}=\ell(\gamma) \int_{0}^{1} \chi_{N}\left(R_{\gamma}(s)\right) \mathrm{d} s=0$ for $\pi$-a.e. $\gamma$. For any arc $\gamma$ for which the integral is null $f\left(R\left(\gamma_{s}\right)\right)$ coincides a.e. in $[0,1]$ with $\tilde{f}\left(R\left(\gamma_{s}\right)\right)$. The same argument shows the sets $\left\{\gamma: f\left(\gamma_{t}\right) \neq\right.$ $\left.\tilde{f}\left(\gamma_{t}\right)\right\} \subset\left\{\gamma: \gamma_{t} \in N\right\}, t=0,1$ are $\boldsymbol{\pi}$-negligible because $\left(\mathrm{e}_{t}\right)_{\sharp} \boldsymbol{\pi} \ll \mathfrak{m}$, which implies that $\boldsymbol{\pi}\left(\left\{\gamma: \gamma_{t} \in N\right\}\right)=\left(\mathrm{e}_{t}\right)_{\sharp} \boldsymbol{\pi}(N)=0$.

(b) If $\tilde{g}$ is a Borel modification of $g$ the set $\{g \neq \tilde{g}\}$ is a m-negligible set; by the previous Claim (a), $g\left(R\left(\gamma_{s}\right)\right)$ coincides $\mathscr{L}^{1}$-a.e. in $[0,1]$ with the Borel map $\tilde{g}\left(R\left(\gamma_{s}\right)\right)$ and it is therefore $\mathscr{L}^{1}$-measurable.

(c) follows immediately by Claim (a) as well, since for $\mathcal{T}_{q}$-a.e. $\operatorname{arc} \gamma \int_{\partial \gamma} f=\int_{\partial \gamma} \tilde{f}$ and $\int_{\gamma} g=$ $\int_{\gamma} \tilde{g}$.

Remark 10.7 (Local Lipschitz constants of d-Lipschitz functions are weak upper gradients). If $f \in$ $\operatorname{Lip}_{b}(X, \tau, \mathrm{d})$ then the local Lipschitz constant lip $f$ is an upper gradient and therefore it is also a $\mathcal{T}_{q^{-}}$ weak upper gradient. An analogous property holds for the d-slopes (notice that the topology $\tau$ does not play any role in the definition)

$$
\left|\mathrm{D}^{ \pm} f\right|(x):=\lim _{\mathrm{d}(y, x) \rightarrow 0} \frac{(f(y)-f(x))_{ \pm}}{\mathrm{d}(y, x)}, \quad|\mathrm{D} f|(x):=\lim _{\mathrm{d}(y, x) \rightarrow 0} \frac{|f(y)-f(x)|}{\mathrm{d}(y, x)}
$$

of an arbitrary d-Lipschitz functions $f \in \mathrm{B}_{b}(X)$ : if $|\mathrm{D} f|$ is $\mathfrak{m}$-measurable (this property is always satisfied if, e.g., $(X, \tau)$ is Souslin, see [9, Lemma 2.6]) then it is a weak upper gradient of $f$.

It is easy to check that for every $\alpha, \beta \in \mathbb{R}$

if $g_{i}$ is a $\mathcal{T}_{q}$-w.u.g. of $f_{i}, i=0,1$, then $|\alpha| g_{0}+|\beta| g_{1}$ is a $\mathcal{T}_{q}$-w.u.g. of $\alpha f_{0}+\beta f_{1}$.

In particular the set $S:=\left\{(f, g): g\right.$ is a $\mathcal{T}_{q}$-w.u.g. of $\left.f\right\}$ is convex.

If we know a priori the integrability of $f$ and the $L^{p}$-summability of $g$ then Definition 10.4 can be formulated in a slightly different way:

Lemma 10.8. Let $f \in L^{1}(X, \mathfrak{m})$ and $g \in L^{p}(X, \mathfrak{m}), g \geq 0 . g$ is a $\mathcal{T}_{q}$-weak upper gradient of $f$ if and only if

$$
\int\left(f\left(\gamma_{1}\right)-f\left(\gamma_{0}\right)\right) \mathrm{d} \boldsymbol{\pi}(\gamma) \leq \int\left(\int_{\gamma} g\right) \mathrm{d} \boldsymbol{\pi}(\gamma) \quad \text { for every } \boldsymbol{\pi} \in \mathcal{T}_{q}^{*}
$$

Equivalently, setting $\pi_{i}:=\left(\mathrm{e}_{i}\right)_{\sharp} \boldsymbol{\pi}, i=0,1$,

$$
\int_{X} f \mathrm{~d}\left(\pi_{1}-\pi_{0}\right) \leq \int_{X} g \mathrm{~d} \mu_{\boldsymbol{\pi}} \quad \text { for every } \boldsymbol{\pi} \in \mathcal{T}_{q}^{*}
$$


Proof. It is clear that (10.8) yields (10.11) simply by integration w.r.t. $\boldsymbol{\pi}$; notice that the integrals in (10.11) (and in (10.12)) are well defined since $\pi_{i}=h_{i} \mathfrak{m}$ and $\mu_{\boldsymbol{\pi}}=h \mathfrak{m}$ for functions $h_{i} \in L^{\infty}(X, \mathfrak{m})$ and $h \in L^{q}(X, \mathfrak{m})$.

Let us prove the converse implication. It is not restrictive to assume that $f, g$ are Borel. By Lemma 10.3, if (10.8) is not true, there exists a nontrivial test plan $\pi \in \mathscr{T}_{q}^{*}$ such that the Borel set $A:=\{\gamma \in$ $\left.\operatorname{RA}(X):\left|\int_{\partial \gamma} f\right|>\int_{\gamma} g\right\}$ satisfies $\boldsymbol{\pi}(A)>0$ (notice that (10.8) is always satisfied on constant arcs). By possible reducing $A$ we can also find $\theta>0$ such that $A^{\prime}:=\left\{\gamma \in \operatorname{RA}(X): \int_{\partial \gamma} f \geq \theta+\int_{\gamma} g\right\}$ satisfies $\boldsymbol{\pi}\left(A^{\prime}\right)>0$. Thus defining $\boldsymbol{\pi}^{\prime}:=\boldsymbol{\pi}_{A_{A^{\prime}}}$, it is immediate to check that $\boldsymbol{\pi}^{\prime} \in \mathcal{T}_{q}^{*}$; a further integration with respect to $\pi^{\prime}$ of the previous inequality yields

$$
\int\left(f\left(\gamma_{1}\right)-f\left(\gamma_{0}\right)\right) \mathrm{d} \boldsymbol{\pi}^{\prime}(\gamma) \geq \theta \boldsymbol{\pi}\left(A^{\prime}\right)+\int\left(\int_{\gamma} g\right) \mathrm{d} \boldsymbol{\pi}^{\prime}(\gamma)
$$

which contradicts (10.11).

Definition 10.9 (Sobolev regularity along a rectifiable arc). We say that a map $f: X \rightarrow \mathbb{R}$ is Sobolev (resp. strictly Sobolev) along an arc $\gamma$ if $f \circ R_{\gamma}$ coincides $\mathscr{L}^{1}$-a.e. in $[0,1]$ (resp. $\mathscr{L}^{1}$-a.e. in $[0,1]$ and in $\{0,1\}$ ) with an absolutely continuous map $f_{\gamma}:[0,1] \rightarrow \mathbb{R}$. In this case, we say that a map $g: X \rightarrow \mathbb{R}$ is a Sobolev upper gradient (S.u.g.) for $f$ along $\gamma$ if $\ell(\gamma) g \circ R_{\gamma} \in L^{1}(0,1)$ and

$$
\left|\frac{\mathrm{d}}{\mathrm{d} t} f_{\gamma}\right| \leq \ell(\gamma) g \circ R_{\gamma} \quad \text { a.e. in }[0,1] .
$$

We can give an intrinsic formulation of the (strict) Sobolev regularity with Sobolev upper gradient $g$ which does not involve the absolutely continuous representative.

Lemma 10.10. Let us suppose that $\gamma \in \mathrm{RA}(X), f, g: X \rightarrow \mathbb{R}$ such that $f \circ R_{\gamma}, \ell(\gamma) g \circ R_{\gamma} \in$ $L^{1}(0,1)$, and $\mathcal{C}$ (resp. $\left.\mathcal{C}_{c}\right)$ is a dense subset of $\mathrm{C}^{1}([0,1])$ (resp. of $\mathrm{C}_{c}^{1}(0,1)$ ).

(a) $f$ is Sobolev along $\gamma$ with Sobolev u.g. $g$ if and only if

$$
\left|-\int_{0}^{1} \varphi^{\prime}(t) f\left(R_{\gamma}(t)\right) \mathrm{d} t\right| \leq \ell(\gamma) \int_{0}^{1}|\varphi(t)| g\left(R_{\gamma}(t)\right) \mathrm{d} t
$$

for every $\varphi \in \mathcal{C}_{c}$.

(b) $f$ is strictly Sobolev along $\gamma$ with Sobolev u.g. $g$ if and only if

$$
\left|\varphi(1) f\left(R_{\gamma}(1)\right)-\varphi(0) f\left(R_{\gamma}(0)\right)-\int_{0}^{1} \varphi^{\prime}(t) f\left(R_{\gamma}(t)\right) \mathrm{d} t\right| \leq \ell(\gamma) \int_{0}^{1}|\varphi(t)| g\left(R_{\gamma}(t)\right) \mathrm{d} t
$$

for every $\varphi \in \mathcal{C}$.

In particular, if $f, g$ are Borel maps, the sets of curves $\gamma \in \mathrm{RA}(X)$ along which $\int_{\gamma}(|f|+g)<\infty$ and $f$ is Sobolev (resp. strictly Sobolev) with S.u.g. $g$ is Borel in $\mathrm{RA}(X)$.

Proof. (a) One implication is obvious. If (10.14) holds for every $\varphi \in \mathcal{C}_{c}$ then it can be extended to every $\varphi \in \mathrm{C}_{c}^{1}(0,1)$. In particular we have

$$
\left|-\int_{0}^{1} \varphi^{\prime}(t) f\left(R_{\gamma}(t)\right) \mathrm{d} t\right| \leq C \sup _{t \in[0,1]}|\varphi(t)| \quad \text { where } \quad C:=\int_{\gamma} g
$$


for every $\varphi \in \mathrm{C}_{c}^{1}(0,1)$, so that the distributional derivative of $f \circ R_{\gamma}$ can be represented by Radon measure $\mu \in \mathcal{M}((0,1))$ with finite total variation. (10.14) also yields

$$
\left|\int_{0}^{1} \varphi \mathrm{d} \mu\right| \leq \ell(\gamma) \int_{0}^{1}|\varphi| g \mathrm{~d} t \quad \text { for every } \varphi \in \mathrm{C}_{c}^{1}(0,1)
$$

so that $\mu=h \mathscr{L}^{1}$ is absolutely continuous w.r.t. $\mathscr{L}^{1}$ with density satisfying $|h| \leq \ell(\gamma) g \circ R_{\gamma} \mathscr{L}^{1}$-a.e. It follows that $f \circ R_{\gamma} \in W^{1,1}(0,1)$ and its absolutely continuous representative $f_{\gamma}$ satisfies (10.13).

(b) follows as in the previous claim (a); from (10.15) it is also not difficult to check that $f_{\gamma}(i)=$ $f \circ R_{\gamma}(i)$ for $i=0$ or $i=1$.

Concerning the last statement, arguing as in the proof of Theorem 3.13(e), it is not difficult to show that every bounded (resp. nonnegative) function $h$ and for every $\psi \in \mathrm{C}([0,1])$ (resp. nonnegative) the real maps

$$
\gamma \mapsto \int_{0}^{1} \psi(t) h(\gamma(t)) \mathrm{d} t \quad \text { are Borel in }\left(\mathrm{C}([0,1] ; X), \tau_{\mathrm{C}}\right)
$$

Since by 3.13(d) the map $\gamma \mapsto R_{\gamma}$ is Borel from $\left(\mathrm{RA}(X), \tau_{\mathrm{A}}\right)$ to $\left(\mathrm{C}([0,1] ; X), \tau_{\mathrm{C}}\right)$ and $\mathcal{C}, \mathcal{C}_{c}$ are countable, we deduce that the sets characterized by the family of inequalities (10.14) or (10.15) are Borel in $\mathrm{RA}(X)$.

Remark 10.11 (Sobolev regularity along $\mathcal{T}_{q}$-almost every arc). By Proposition 10.6(a), it is easy to check that the properties to be Sobolev or strictly Sobolev along $\mathcal{T}_{q}$-almost every arc with S.u.g. $g$ are invariant with respect to modification of $f$ and $g$ in $\mathfrak{m}$-negligible sets, and thus they make sense for Lebesgue classes. It is also not restrictive to consider only arcs $\gamma \in \mathrm{RA}_{0}(X)$.

In the next Theorem we prove that existence of a $\mathcal{T}_{q}$-weak upper gradient yields strict Sobolev regularity along $\mathcal{T}_{q}$-almost every arc. This property is based on a preliminary lemma which provides this property for stretchable plans in $\mathcal{T}_{q}^{*}$.

Lemma 10.12. Assume that $\boldsymbol{\pi} \in \mathcal{T}_{q}^{*}$ is a stretchable plan and that $g: X \rightarrow[0, \infty]$ is a $\mathcal{T}_{q}$-weak upper gradient of a m-measurable function $f: X \rightarrow \mathbb{R}$. Then $f$ is strictly Sobolev along $\pi$-almost every arc with S.u.g. g, i.e. (10.15) or equivalently

$$
\left|\frac{\mathrm{d}}{\mathrm{d} t} f_{\gamma}\right| \leq \ell(\gamma) g \circ R_{\gamma} \quad \text { a.e. in }[0,1], \quad f_{\gamma}(i)=f\left(R_{\gamma}(i)\right) \quad i \in\{0,1\}
$$

hold for $\pi$-almost every $\gamma \in \mathrm{RA}(X)$.

Proof. Arguing as in Proposition 10.6 it is not restrictive to assume that $f, g$ are Borel function. Since $\pi \in \mathcal{T}_{q}^{*}$ we know that there exists a compact set $\mathcal{K} \subset \mathrm{RA}(X) \boldsymbol{\pi}$ satisfying (10.4). The stretchable condition (10.5) and an obvious change of variables related to the maps $R_{\gamma}^{s \rightarrow t}$ of (10.1) yields for every $s<t$ in $[0,1]$ and for $\pi$-almost every $\gamma \in \mathcal{K}$,

$$
\left|f\left(R_{\gamma}(t)\right)-f\left(R_{\gamma}(s)\right)\right| \leq(t-s) \ell(\gamma) \int_{0}^{1} g\left(R_{\gamma}^{s \rightarrow t}(r)\right) \mathrm{d} r=\ell(\gamma) \int_{s}^{t} g\left(R_{\gamma}(r)\right) \mathrm{d} r
$$

since $\operatorname{Restr}_{s}^{t}(\boldsymbol{\pi}) \in \mathcal{T}_{q}$. We apply Fubini's Theorem to the product measure $\mathscr{L}^{2} \otimes \boldsymbol{\pi}$ in $(0,1)^{2} \times \mathcal{K}$ and we use the fact that the maps characterizing the inequality (10.17) are jointly Borel with respect 
to $(s, t, \gamma) \in(0,1)^{2} \times \mathcal{K}$ : here we use the continuity of $R$ from $\mathcal{K}$ to $\operatorname{BVC}_{c}([0,1] ; X)$ endowed with the topology $\tau_{\mathrm{C}}$. It follows that for $\pi$-a.e. $\gamma$ the function $f$ satisfies

$$
\left|f\left(R_{\gamma}(t)\right)-f\left(R_{\gamma}(s)\right)\right| \leq \ell(\gamma) \int_{s}^{t} g\left(R_{\gamma}(r)\right) \mathrm{d} r \quad \text { for } \mathscr{L}^{2} \text {-a.e. }(t, s) \in(0,1)^{2} .
$$

An analogous argument shows that for $\pi$-a.e. $\gamma$

$$
\left\{\begin{array}{l}
\left|f\left(R_{\gamma}(s)\right)-f\left(\gamma_{0}\right)\right| \leq \ell(\gamma) \int_{0}^{s} g\left(R_{\gamma}(r)\right) \mathrm{d} r \\
\left|f\left(\gamma_{1}\right)-f\left(R_{\gamma}(s)\right)\right| \leq \ell(\gamma) \int_{s}^{1} g\left(R_{\gamma}(r)\right) \mathrm{d} r
\end{array} \quad \text { for } \mathscr{L}^{1} \text {-a.e. } s \in(0,1) .\right.
$$

Since $g \circ R_{\gamma} \in L^{1}(0,1)$ for $\pi$-a.e. $\gamma \in \mathcal{K}$ with $\ell(\gamma)>0$,, by the next Lemma 10.13 it follows that $f \circ R_{\gamma} \in W^{1,1}(0,1)$ for $\pi$-a.e. $\gamma$ and (understanding the derivative of $f \circ R_{\gamma}$ as the distributional one)

$$
\left|\frac{\mathrm{d}}{\mathrm{d} t}\left(f \circ R_{\gamma}\right)\right| \leq \ell(\gamma) g \circ R_{\gamma} \quad \mathscr{L}^{1} \text {-a.e. in }(0,1), \text { for } \pi \text {-a.e. } \gamma \text {. }
$$

We conclude that $f \circ R_{\gamma} \in W^{1,1}(0,1)$ for $\pi$-a.e. $\gamma$, and therefore it admits an absolutely continuous representative $f_{\gamma}$ for which (10.16) holds; moreover, by (10.18), it is immediate to check that $f(\gamma(t))=f_{\gamma}(t)$ for $t \in\{0,1\}$ and $\pi$-a.e. $\gamma$.

Lemma 10.13. Let $f:(0,1) \rightarrow \mathbb{R}$ and $g \in L^{q}(0,1)$ nonnegative satisfy

$$
|f(t)-f(s)| \leq\left|\int_{s}^{t} g(r) \mathrm{d} r\right| \text { for } \mathscr{L}^{2} \text {-a.e. }(s, t) \in(0,1) \times(0,1) .
$$

Then $f \in W^{1, q}(0,1)$ and $\left|f^{\prime}\right| \leq g \mathscr{L}^{1}$-a.e. in $(0,1)$.

We refer to [8, Lemma 2.1] for the proof.

We can considerably refine Lemma 10.12 , by removing the assumption that $\pi$ is stretchable and by considering arbitrary nonparametric dynamic plans in $\mathcal{T}_{q}$.

Theorem 10.14. Assume that $g: X \rightarrow[0, \infty]$ is a $\mathcal{T}_{q}$-weak upper gradient of a $\mathfrak{m}$-measurable function $f: X \rightarrow \mathbb{R}$. Then $f$ is strictly Sobolev and satisfies (10.16) along $\mathcal{T}_{q}$-almost every arc.

Proof. By Lemma 10.3 it is sufficient to prove the property for every $\pi \in \mathcal{T}_{q}^{*}$, so that we can also assume that there exists a compact set $\mathcal{K} \subset \mathrm{RA}(X)$ satisfying (10.4).

For every $r \in[0,1 / 3]$ and $s \in[2 / 3,1]$ we consider the rescaled plans

$$
\boldsymbol{\pi}_{r}^{+}:=\left(\operatorname{Restr}_{r}^{1}\right)_{\sharp} \boldsymbol{\pi}, \quad \boldsymbol{\pi}_{s}^{-}:=\left(\operatorname{Restr}_{0}^{s}\right)_{\sharp}(\boldsymbol{\pi})
$$

which form two continuous (thus Borel) collections depending on $r \in[0,1 / 3], s \in[2 / 3,1]$. We then set

$$
\boldsymbol{\pi}^{+}:=3 \int_{0}^{1 / 3} \boldsymbol{\pi}_{r}^{+} \mathrm{d} r, \quad \boldsymbol{\pi}^{-}:=3 \int_{2 / 3}^{1} \boldsymbol{\pi}_{s}^{-} \mathrm{d} s .
$$

Notice that we can equivalently characterize $\pi^{+}, \pi^{-}$as the push forward measures of

$$
\boldsymbol{\sigma}^{+}:=\left.3 \mathscr{L}^{1}\right|_{(0,1 / 3)} \otimes \boldsymbol{\pi} \text { and } \boldsymbol{\sigma}^{-}:=\left.3 \mathscr{L}^{1}\right|_{(2 / 3,1)} \otimes \boldsymbol{\pi}
$$

through the continuous maps $(r, \gamma) \mapsto \operatorname{Restr}_{r}^{1}(\gamma)$ and $(s, \gamma) \mapsto \operatorname{Restr}_{0}^{s}(\gamma) \operatorname{respectively:~}^{\circ}$

$$
\boldsymbol{\pi}^{+}=\left(\operatorname{Restr}_{.}^{1}\right)_{\sharp}\left(\left.3 \mathscr{L}^{1}\right|_{(0,1 / 3)} \otimes \boldsymbol{\pi}\right), \quad \boldsymbol{\pi}^{-}=\left(\operatorname{Restr}_{0}^{\cdot}\right)_{\sharp}\left(\left.3 \mathscr{L}^{1}\right|_{(2 / 3,1)} \otimes \boldsymbol{\pi}\right) . .
$$


Let us check that $\boldsymbol{\pi}^{ \pm}$belong to $\mathcal{T}_{q}^{*}$ and are stretchable. We only consider $\boldsymbol{\pi}^{+}$, since the argument for $\boldsymbol{\pi}^{-}$is completely analogous. Recalling Lemma 8.3, for every nonnegative $f \in \mathrm{C}_{b}(X)$ we have

$$
\begin{aligned}
\iint_{\gamma} f \mathrm{~d} \boldsymbol{\pi}^{+}(\gamma) & \stackrel{(10.22)}{=} 3 \int_{0}^{1 / 3}\left(\iint_{\gamma} f \mathrm{~d} \boldsymbol{\pi}_{r}^{+}(\gamma)\right) \mathrm{d} r \stackrel{(10.2)}{=} 3 \int_{0}^{1 / 3}\left(\int \ell(\gamma) \int_{r}^{1} f\left(R_{\gamma}(s)\right) \mathrm{d} s \mathrm{~d} \boldsymbol{\pi}(\gamma)\right) \mathrm{d} r \\
& \leq 3 \int_{0}^{1 / 3}\left(\iint_{\gamma} f \mathrm{~d} \boldsymbol{\pi}(\gamma)\right) \mathrm{d} r \stackrel{(8.9)}{\leq}\|f\|_{L^{p}} \operatorname{Bar}_{q}(\boldsymbol{\pi})
\end{aligned}
$$

which shows that $\operatorname{Bar}_{q}\left(\boldsymbol{\pi}^{+}\right) \leq \operatorname{Bar}_{q}(\boldsymbol{\pi})$.

Let us now prove that $\left(\hat{\mathrm{e}}_{s}\right)_{\sharp} \boldsymbol{\pi}^{+} \ll \mathfrak{m}$ with density in $L^{q}(X, \mathfrak{m})$ for every $s \in[0,1]$; setting $\ell_{o}:=\min _{\mathcal{K}} \ell>0$, if $s<1$ we have

$$
\begin{aligned}
\int f \mathrm{~d}\left(\hat{\mathrm{e}}_{s}\right)_{\sharp} \boldsymbol{\pi}^{+} & =\int f\left(R_{\gamma}(s)\right) \mathrm{d} \boldsymbol{\pi}^{+}(\gamma) \stackrel{(10.22)}{=} 3 \int_{0}^{1 / 3}\left(\int f\left(R_{\gamma}(s)\right) \mathrm{d} \boldsymbol{\pi}_{r}^{+}(\gamma)\right) \mathrm{d} r \\
& \stackrel{(10.23)}{=} 3 \int_{0}^{1 / 3}\left(\int f\left(R_{\gamma}^{r \rightarrow 1}(s)\right) \mathrm{d} \boldsymbol{\pi}(\gamma)\right) \mathrm{d} r=3 \int\left(\int_{0}^{1 / 3} f\left(R_{\gamma}(r(1-s)+s)\right) \mathrm{d} r\right) \mathrm{d} \boldsymbol{\pi}(\gamma) \\
& =\frac{3}{1-s} \int\left(\int_{s}^{s+(1-s) / 3} f\left(R_{\gamma}(\theta)\right) \mathrm{d} \theta\right) \mathrm{d} \boldsymbol{\pi}(\gamma) \\
& \leq \frac{3}{\ell_{o}(1-s)} \iint_{\gamma} f \mathrm{~d} \boldsymbol{\pi}(\gamma) \leq \frac{3}{\ell_{o}(1-s)}\|f\|_{L^{p}} \operatorname{Bar}_{q}(\boldsymbol{\pi})
\end{aligned}
$$

On the other hand, if $s=1$, we can use the fact that $\left(\mathrm{e}_{1}\right)_{\sharp} \boldsymbol{\pi}_{r}=\left(\mathrm{e}_{1}\right)_{\sharp} \boldsymbol{\pi}$

$$
\begin{aligned}
\int f\left(\gamma_{1}\right) \mathrm{d} \boldsymbol{\pi}^{+}(\gamma) & =3 \int_{0}^{1 / 3}\left(\int f(\gamma(1)) \mathrm{d} \boldsymbol{\pi}_{r}^{+}(\gamma)\right) \mathrm{d} r=3 \int_{0}^{1 / 3}\left(\int f(\gamma(1)) \mathrm{d} \boldsymbol{\pi}(\gamma)\right) \mathrm{d} r \\
& =\int f \mathrm{~d}\left(\mathrm{e}_{1}\right)_{\sharp} \boldsymbol{\pi},
\end{aligned}
$$

so that $\left(\mathrm{e}_{1}\right)_{\sharp} \boldsymbol{\pi}^{+}=\left(\mathrm{e}_{1}\right)_{\sharp} \boldsymbol{\pi}$ which has an $L^{q}$ density w.r.t. $\mathfrak{m}$.

Let us now select a Borel representative of $f$. Applying Lemma 10.12 we know that $f$ is Sobolev along $\boldsymbol{\pi}^{+}$and $\boldsymbol{\pi}^{-}$-almost every arc. Recalling the representation result (10.23) and applying Fubini's Theorem, we can find a $\pi$-negligible set $N \subset \mathcal{K}$ such that for every $\gamma \in \mathcal{K} \backslash N$ the map $f$ is

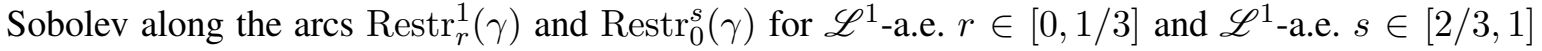
and (10.16) holds. Choosing arbitrarily $r \in[0,1 / 3]$ and $s \in[2 / 3,1]$ so that such a property holds, since the absolutely continuous representative $f_{\gamma}$ should coincide along the curve $t \mapsto R_{\gamma}(t)$ in the interval $[r, s]$, one immediately sees that $f$ is Sobolev along $\gamma$ and (10.16) holds as well. We conclude that $f$ is Sobolev along $\boldsymbol{\pi}$-a.e. arc and since $\boldsymbol{\pi}$ is arbitrary in $\mathcal{T}_{q}^{*}$ we get the thesis.

Remark 10.15 (Equivalent formulation). By a similar argument we obtain an equivalent formulation of the weak upper gradient property when $f$ is Sobolev along $\mathcal{T}_{q}$-almost every arc: a function $g$ satisfying $\int_{\gamma} g<\infty$ for $\mathcal{T}_{q}$-almost every arc $\gamma$ is a $\mathcal{T}_{q}$-weak upper gradient of $f$ if and only if (10.15) holds for every $\varphi$ in a dense subset of $\mathrm{C}^{1}([0,1])$ and $\mathcal{T}_{q}$-almost every arc $\gamma$, or, equivalently, the function $f_{\gamma}$ of Definition 10.9 satisfies $(10.16) \mathcal{T}_{q}$-almost everywhere.

\subsection{The link with $\operatorname{Mod}_{p}$-weak upper gradients}

In this section we will show that the definition of $\mathcal{T}_{q}$-weak upper gradient can be equivalently stated in terms of $\operatorname{Mod}_{p}$, as in the Newtonian approach to metric Sobolev spaces. Part of the results stated 
here could also be derived as a consequence of the identification Theorem of Section 11, so we will just sketch the main ideas.

First of all we can associate a $q$-barycentric entropy to plans in $\mathcal{T}_{q}$ : we consider the measure $\tilde{\mu}_{\boldsymbol{\pi}} \in \mathcal{M}_{+}(X)$ defined by

$$
\int_{X} f \mathrm{~d} \tilde{\mu}_{\boldsymbol{\pi}}:=\int\left(f\left(\gamma_{0}\right)+f\left(\gamma_{1}\right)+\int_{\gamma} f\right) \mathrm{d} \boldsymbol{\pi}(\gamma)=\int\left(\int f \mathrm{~d} \tilde{\mathrm{M}} \gamma\right) \mathrm{d} \boldsymbol{\pi}(\gamma)
$$

where $\tilde{\mathrm{M}}: \operatorname{RA}(X) \rightarrow \mathcal{M}_{+}(X)$ has been defined in (7.12). We then set

$$
\frac{1}{q} \widetilde{\operatorname{Bar}}_{q}^{q}(\boldsymbol{\pi}):=\mathscr{L}^{q}\left(\tilde{\mu}_{\boldsymbol{\pi}} \mid \mathfrak{m}\right)=\frac{1}{q} \int_{X} h^{q} \mathrm{~d} \mathfrak{m} \quad \text { if } \tilde{\mu}_{\boldsymbol{\pi}}=h \mathfrak{m} .
$$

and it is easy to check that

$$
\pi \in \mathcal{T}_{q} \text { if and only if } \widetilde{\operatorname{Bar}}_{q}(\boldsymbol{\pi})<\infty .
$$

It is clear that $\widetilde{\operatorname{Bar}_{q}}(\boldsymbol{\pi}) \geq \operatorname{Bar}_{q}(\boldsymbol{\pi})$ for every dynamic plan $\boldsymbol{\pi}$. Arguing as in the proof of Lemma 8.5 one can also see that for every $k \geq 0$

$$
\text { if }(X, \mathrm{~d}) \text { is complete then the set }\left\{\boldsymbol{\pi} \in \mathcal{M}_{+}(\operatorname{RA}(X)): \widetilde{\operatorname{Bar}}_{q}(\boldsymbol{\pi}) \leq k\right\} \text { is compact. }
$$

By duality we obtain the corresponding notion of content

$$
\frac{1}{p} \widetilde{\operatorname{Cont}}_{p}(\Gamma):=\sup \left\{\boldsymbol{\pi}(\Gamma)-\frac{1}{q} \widetilde{\operatorname{Bar}}_{q}^{q}(\boldsymbol{\pi}): \boldsymbol{\pi} \in \mathcal{T}_{q}\right\}
$$

and we can obtain an important characterization of $\widetilde{\operatorname{Mod}}_{p}$, as for Theorems 9.1 and 9.2:

Theorem 10.16. (a) If $\Gamma$ is a compact subset of $\mathrm{RA}(X)$ then

$$
\widetilde{\operatorname{Cont}}_{p}^{p}(\Gamma)=\widetilde{\operatorname{Mod}}_{p}(\Gamma)=\widetilde{\operatorname{Mod}}_{p, c}(\Gamma) .
$$

(b) $\widetilde{\operatorname{Mod}}_{p}$ is a $\mathscr{K}\left(\mathrm{RA}(X), \tau_{\mathrm{A}}\right)$-Choquet capacity in $\mathrm{RA}(X)$.

(c) For every universally measurable $\Gamma \subset \mathrm{RA}(X)$

$$
\widetilde{\operatorname{Cont}}_{p}(\Gamma)=\sup \left\{\widetilde{\operatorname{Cont}}_{p}(\mathrm{~K}): \mathrm{K} \subset \Gamma, \mathrm{K} \text { compact }\right\} .
$$

(d) If $(X, \mathrm{~d})$ is complete and $(X, \tau)$ is Souslin then every $\mathscr{B}\left(\mathrm{RA}(X), \tau_{\mathrm{A}}\right)$-analytic set $\Gamma$ is $\widetilde{\operatorname{Mod}}_{p^{-}}$ capacitable and satisfies $\widetilde{\operatorname{Mod}}_{p}(\Gamma)={\widetilde{\operatorname{Cont}_{p}}}_{p}(\Gamma)$. In particular $\Gamma$ is $\widetilde{\operatorname{Mod}}_{p}$-negligible if and only if it is $\mathcal{T}_{q}$-negligible.

We leave the proof to the reader: Claim (a) is based on the same min-max argument of Theorem 9.1 (replacing integration w.r.t. $\nu_{\gamma}$ with integration w.r.t. $\tilde{\nu}_{\gamma}$ ), Claims (b-d) can be obtained by arguing as in Theorem 9.2. In fact, the proofs would be slightly easier, since compactness of sublevels of $\widetilde{\mathrm{Bar}}_{q}$ and tightness of $\widetilde{\operatorname{Mod}}_{p}$ behave better than the corresponding properties for $\operatorname{Bar}_{q}$ and $\operatorname{Mod}_{p}$. It would also be possible to derive the proofs by a general duality between $\operatorname{Mod}_{p}$ and a corresponding notion of content in $\mathcal{M}_{+}(X)$, see $[2, \S 5]$.

Let us now observe that if we consider only Sobolev regularity along arcs, we can improve Theorem 10.14 . 


\section{Proposition 10.17.}

(a) If $g \in L^{p}(X, \mathfrak{m}), g \geq 0$, is a $\mathcal{T}_{q}$-weak upper gradient of a $\mathfrak{m}$-measurable function $f: X \rightarrow \mathbb{R}$, then $f$ is Sobolev with S.u.g. $g$ along $\mathcal{B}_{q}$-almost every arc; (10.13) holds for $\mathcal{B}_{q}$-almost every $\gamma \in \operatorname{RA}(X)$.

(b) If moreover $(X, \mathrm{~d})$ is complete and $(X, \tau)$ is Souslin, then $f$ is Sobolev with S.u.g. $g$ along $\operatorname{Mod}_{p}$-almost every arc and (10.13) holds $\operatorname{Mod}_{p}$-a.e.

Proof. (a) It is not restrictive to assume that $f$ and $g$ are Borel. Let us show that for every plan $\boldsymbol{\pi} \in \mathcal{B}_{q} f$ is Sobolev along $\boldsymbol{\pi}$-a.e. arc $\gamma$ and (10.13) holds $\boldsymbol{\pi}$-a.e. Since $\boldsymbol{\pi}$ is Radon and both the properties trivially holds along constant arcs, it is not restrictive to assume that $\boldsymbol{\pi}$ it is concentrated on a compact set $\mathrm{K} \subset \mathrm{RA}_{0}(X)$ where $\ell$ is continuous. In particular the map $T:(r, \gamma) \mapsto \operatorname{Restr}_{1-r}^{r}(\gamma)$ is continuous in $[0,1 / 3] \times \mathrm{K}$. Arguing as in the proof of Theorem 10.14 we define

$$
\boldsymbol{\pi}_{r}:=\left(\operatorname{Restr}_{1-r}^{r}\right)_{\sharp} \boldsymbol{\pi}, \quad \tilde{\boldsymbol{\pi}}:=3 \int_{0}^{1 / 3} \boldsymbol{\pi}_{r} \mathrm{~d} r=T_{\sharp}\left(\left.3 \mathscr{L}^{1}\right|_{(0,1 / 3)} \otimes \boldsymbol{\pi}\right),
$$

and by calculations similar to (10.24) and (10.25) we can check that $\tilde{\boldsymbol{\pi}} \in \mathcal{T}_{q}$. By Theorem 10.14 we deduce that $f$ is Sobolev along $\tilde{\boldsymbol{\pi}}$-a.e. arc and (10.13) holds for $\tilde{\boldsymbol{\pi}}$-a.e. $\gamma$. Applying Fubini's Theorem we can find a $\pi$-negligible Borel set $\mathrm{N} \subset \mathrm{RA}(X)$ such that for every $\gamma \in \mathrm{RA}(X) \backslash \mathrm{N} f$ is Sobolev

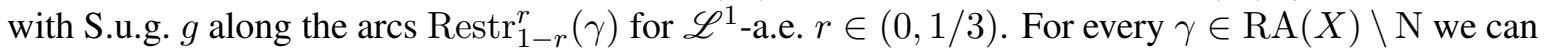
thus find a vanishing sequence $r_{n} \downarrow 0$ such that $f$ is Sobolev and (10.13) holds along $\operatorname{Restr}_{1-r_{n}}^{r_{n}}(\gamma)$. We can thus pass to the limit and obtain the same properties along $\gamma$.

(b) As in the previous Claim, it is not restrictive to assume $f, g$ Borel; by Remark 10.5 we can also suppose that $f$ is bounded. Let us consider a countable dense subset $\mathrm{C}_{c}$ of $\mathrm{C}_{c}^{1}(0,1)$ and let us define the sets

$$
\begin{aligned}
& A_{0}:=\left\{\gamma \in \mathrm{RA}_{0}(X): \int_{\gamma} g<\infty\right\} \\
& B_{0}:=\left\{\gamma \in A_{0}:\left|\int_{0}^{1} \varphi^{\prime}(t) f\left(R_{\gamma}(t)\right) \mathrm{d} t\right| \leq \ell(\gamma) \int_{0}^{1}|\varphi(t)| g\left(R_{\gamma}(t)\right) \mathrm{d} t \quad \text { for every } \varphi \in \mathcal{C}_{c}\right\} .
\end{aligned}
$$

By Theorem 3.13(e) $A_{0}$ is a Borel set; Proposition 7.2(b) shows that $\operatorname{Mod}_{p}\left(\operatorname{RA}_{0}(X) \backslash A_{0}\right)=0$. By Lemma 10.10 for every arc $\gamma \in A_{0}, f$ is Sobolev along $\gamma$ with S.u.g. $g$ if and only if $\gamma \in B_{0}$. Lemma 10.10 also shows that $A_{0} \backslash B_{0}$ is Borel. Since by Claim (a) we know that $\operatorname{Cont}_{p}\left(A_{0} \backslash B_{0}\right)=0$, we get $\operatorname{Mod}_{p}\left(A_{0} \backslash B_{0}\right)=0$ by Theorem 9.2(d).

According to the Definition 10.9, Proposition 10.17 ensures that for $\operatorname{Mod}_{p}$-a.e. arc $\gamma$ a function $f$ with $\mathcal{T}_{q}$-w.u.g. in $L^{p}(X, \mathfrak{m})$ coincides $\mathscr{L}^{1}$-a.e. with an absolutely continuous function $f_{\gamma}$. We can in fact prove a much better result, which establishes a strong connection with the theory of Newtonian Sobolev spaces.

Theorem 10.18 (Good representative). Let us suppose that $(X, \mathrm{~d})$ is complete and $(X, \tau)$ is Souslin. Every $\mathfrak{m}$-measurable function $f$ with a $\mathcal{T}_{q}$-w.u.g. $g \in L^{p}(X, \mathfrak{m})$ admits a Borel $\mathfrak{m}$-representative $\tilde{f}$ such that $\tilde{f} \circ R_{\gamma}$ is absolutely continuous with S.u.g. $g$ along $\operatorname{Mod}_{p}$-a.e. arc $\gamma$ (and a fortiori along $\mathcal{T}_{q}$-a.e. arc). 
Proof. As usual, it is not restrictive to assume that $f, g$ are Borel maps, $f$ bounded. We will also denote by $f_{\gamma}$ the absolutely continuous representative of $f \circ R_{\gamma}$ whenever $f$ is Sobolev along $\gamma$.

Claim 1: There exists $h \in \mathcal{L}_{+}^{p}(X, \mathfrak{m})$, such that

$f$ is Sobolev with S.u.g. $g$ along all the arcs of $H:=\left\{\gamma \in \mathrm{RA}_{0}(X): \int_{\gamma} h<\infty\right\}$.

$f$ is strictly Sobolev with S.u.g. $g$ along all the arcs of

$$
H_{0}:=\left\{\gamma \in \mathrm{RA}_{0}(X): h\left(\gamma_{0}\right)+h\left(\gamma_{1}\right)+\int_{\gamma} h<\infty\right\} .
$$

Notice that $H_{0} \subset H, \operatorname{Mod}_{p}\left(\mathrm{RA}_{0}(X) \backslash H\right)=0$, and $\widetilde{\operatorname{Mod}}_{p}\left(\operatorname{RA}_{0}(X) \backslash H_{0}\right)=0$.

By Proposition 10.17 and Proposition 7.2(b) we can find a Borel function $h^{\prime} \in \mathcal{L}_{+}^{p}(X, \mathfrak{m})$ such that $f$ is Sobolev with S.u.g. $g$ along all the arcs of $H^{\prime}:=\left\{\gamma \in \operatorname{RA}_{0}(X): \int_{\gamma} h^{\prime}<\infty\right\}$. Notice that $\operatorname{Mod}_{p}\left(\operatorname{RA}_{0}(X) \backslash H^{\prime}\right)=0$.

In order to get (10.37) we argue as in the proof of Proposition 10.17(b): we fix a countable set $\mathcal{C}$ dense in $\mathrm{C}^{1}([0,1])$ and we consider the sets

$$
\begin{aligned}
& A:=\left\{\gamma \in \operatorname{RA}(X): \int_{\gamma} g<\infty\right\}, \\
& B:=\left\{\gamma \in A:\left|\varphi(1) f\left(R_{\gamma}(1)\right)-\varphi(0) f\left(R_{\gamma}(0)\right)-\int_{0}^{1} \varphi^{\prime}(t) f\left(R_{\gamma}(t)\right) \mathrm{d} t\right| \leq\right. \\
&\left.\qquad(\gamma) \int_{0}^{1}|\varphi(t)| g\left(R_{\gamma}(t)\right) \mathrm{d} t \quad \text { for every } \varphi \in \mathcal{C}\right\},
\end{aligned}
$$

By Theorem 3.13(e) $A$ is a Borel set; Proposition 7.2(b) shows that $\widetilde{\operatorname{Mod}}_{p}(\operatorname{RA}(X) \backslash A)=0$. By Lemma 10.10 for every arc $\gamma \in A, f$ is Sobolev along $\gamma$ and (10.16) holds if and only if $\gamma \in B$, so that $\widetilde{\operatorname{Cont}_{p}}(A \backslash B)=0$. Lemma 10.10 also shows that $B$ is Borel, so that $\widetilde{\operatorname{Mod}}_{p}(\operatorname{RA}(X) \backslash B)=0$ by Theorem 10.16(d). We can eventually apply Proposition 7.2 to find $h_{0}^{\prime} \in \mathcal{L}_{+}^{p}(X, \mathfrak{m})$ such that $f$ is Sobolev with S.u.g. $g$ along all the arcs of $H_{0}^{\prime}:=\left\{\gamma \in \mathrm{RA}_{0}(X): h_{0}^{\prime}\left(\gamma_{0}\right)+h_{0}^{\prime}\left(\gamma_{1}\right)+\int_{\gamma} h_{0}^{\prime}<\infty\right\}$ We can eventually set $h:=h^{\prime}+h_{0}^{\prime}$ and define the sets $H$ and $H_{0}$ accordingly.

Claim 2: If $\gamma, \gamma^{\prime} \in H$ and $R_{\gamma}(r)=R_{\gamma^{\prime}}\left(r^{\prime}\right)$ for some $r, r^{\prime} \in[0,1]$ then $f_{\gamma}(r)=f_{\gamma}\left(r^{\prime}\right)$.

Let us argue by contradiction assuming that there exist $\gamma, \gamma^{\prime} \in H$ and $r, r^{\prime} \in[0,1]$ such that $R_{\gamma}(r)=$ $R_{\gamma^{\prime}}\left(r^{\prime}\right)=x$ but $f_{\gamma}(r) \neq f_{\gamma^{\prime}}\left(r^{\prime}\right)$. Up to a possible inversion of the orientation of $\gamma$ or $\gamma^{\prime}$ it is not restrictive to assume that $r>0$ and $r^{\prime}<1$. We can then consider the curve $\gamma^{\prime \prime}$ obtained by gluing $\gamma_{-}=\operatorname{Restr}_{0}^{r}(\gamma)$ and $\gamma_{+}=\operatorname{Restr}_{r^{\prime}}^{1}\left(\gamma^{\prime}\right)$, with $\ell\left(\gamma^{\prime \prime}\right)=r \ell(\gamma)+\left(1-r^{\prime}\right) \ell\left(\gamma^{\prime}\right)$. Clearly $\gamma^{\prime \prime} \in \operatorname{RA}(X)$ and $\int_{\gamma^{\prime \prime}} h=\int_{\gamma_{-}} h+\int_{\gamma_{+}} h<\infty$, so that $\gamma^{\prime \prime} \in H$ as well. Moreover, if $r^{\prime \prime}=r \ell(\gamma) / \ell\left(\gamma^{\prime \prime}\right)$ we have $R_{\gamma^{\prime \prime}}^{0 \rightarrow r^{\prime \prime}}(t)=R_{\gamma}^{0 \rightarrow r}(t)$ and $R_{\gamma^{\prime \prime}}^{r^{\prime \prime} \rightarrow 1}(t)=R_{\gamma^{\prime}}^{r^{\prime} \rightarrow 1}(t)$ for every $t \in[0,1]$. It follows that $f_{\gamma^{\prime \prime}}(t)=$ $f_{\gamma}\left(r t / r^{\prime \prime}\right)$ for $t \in\left[0, r^{\prime \prime}\right]$ and $f_{\gamma^{\prime \prime}}(t)=f_{\gamma^{\prime}}\left(r^{\prime}+\left(1-r^{\prime}\right)\left(t-r^{\prime \prime}\right) /\left(1-r^{\prime \prime}\right)\right)$ so that $\lim _{t \uparrow r_{2}} f_{\gamma^{\prime \prime}}(t)=$ $f_{\gamma}(r) \neq \lim _{t \downarrow r_{2}} f_{\gamma^{\prime \prime}}(t)=f_{\gamma^{\prime}}\left(r^{\prime}\right)$, which conflicts with the fact that $f_{\gamma^{\prime \prime}}$ is absolutely continuous.

Claim 3: Let us set

$$
\tilde{f}(x):= \begin{cases}f_{\gamma}(r) & \text { if } x=R_{\gamma}(r) \text { for some } \gamma \in H \text { and } r \in[0,1] \\ f(x) & \text { otherwise }\end{cases}
$$


Then $\tilde{f}$ is well defined, $\tilde{f}\left(R_{\gamma}\right) \equiv f_{\gamma}$ for every $\gamma \in H$, and $\tilde{f}(x)=f(x)$ in $\{x \in X: h(x)<\infty\}$. In particular $\{\tilde{f} \neq f\}$ is $\mathfrak{m}$-negligible and $\tilde{f}=f_{\gamma}$ along $\operatorname{Mod}_{p}$-a.e. arc (and a fortiori along $\mathcal{B}_{q}$ and $\mathcal{T}_{q}$-a.e. arc).

The facts that $\tilde{f}$ is well defined and $\tilde{f}\left(R_{\gamma}\right) \equiv f_{\gamma}$ for every $\gamma \in H$ follow directly from the previous claim. Let us now argue by contradiction and let us suppose that there exists $x \in X$ with $\tilde{f}(x) \neq f(x)$ and $h(x)<\infty$. By definition of $\tilde{f}$ there exists an arc $\gamma \in H$ and $r \in[0,1]$ such that $R_{\gamma}(r)=x$. Since $\gamma \in H$ we know that $\int_{\gamma} h<\infty$ : we can thus find $s \in[0,1] \backslash r$ such that $h\left(R_{\gamma}(s)\right)<\infty$. Assuming that $r<s$ (otherwise we switch the order of $r$ and $s$ ), we can consider the $\operatorname{arc} \gamma^{\prime}:=\operatorname{Restr}_{r}^{s}(\gamma)$ which satisfies $\int_{\gamma^{\prime}} h \leq \int_{\gamma} h<\infty$ and $h\left(R_{\gamma^{\prime}}(0)\right)=h\left(R_{\gamma}(r)\right)=h(x)<\infty$ and $h\left(R_{\gamma^{\prime}}(1)\right)=h\left(R_{\gamma}(s)\right)<$ $\infty$. We deduce that $\gamma^{\prime} \in H_{0}$ so that $f$ is strictly Sobolev along $\gamma^{\prime}$ and therefore $\tilde{f}(x)=\tilde{f}\left(R_{\gamma^{\prime}}(0)\right)=$ $f_{\gamma^{\prime}}\left(R_{\gamma^{\prime}}(0)\right)=f\left(R_{\gamma^{\prime}}(0)\right)=f(x)$, a contradiction.

Let us apply the previous representation Theorem to prove the equivalence of the notion of $\mathcal{T}_{q^{-}}$ w.u.g. with the "Newtonian" one introduced in [58].

Definition 10.19 (Newtonian weak upper gradient). Let $f \in \mathcal{L}^{p}(X, \mathfrak{m})$. We say that $f$ belongs to the Newtonian space $N^{1, p}(\mathbb{X})$ if $f$ is absolutely continuous along $\operatorname{Mod}_{p}$-a.e. arc $\gamma \in \mathrm{RA}_{0}(X)$ and there exists a nonnegative $g \in L^{p}(X, \mathfrak{m})$ such that

$$
\left|\int_{\partial \gamma} f\right| \leq \int_{\gamma} g \text { for } \operatorname{Mod}_{p} \text {-a.e. arc } \gamma \in \mathrm{RA}_{0}(X) .
$$

In this case, we say that $g$ is a $N^{1, p}$-weak upper gradient of $f$.

Functions with $\operatorname{Mod}_{p}$-weak upper gradient have the important Beppo-Levi property of being absolutely continuous along $\operatorname{Mod}_{p^{-}}$-a.e. arc $\gamma$. Because of the implication (10.6), functions with $\operatorname{Mod}_{p^{-}}$ weak upper gradient have also $\mathcal{T}_{q}$-weak upper gradient. A priori there is an important difference between the two definitions, since Definition 10.19 is not invariant w.r.t. modifications of $f$ in a $\mathfrak{m}$ negligible set. However, as an application of Theorem 10.18, we can show that these two notions are essentially equivalent modulo the choice of a representative in the equivalence class:

Corollary 10.20. Let us suppose that $\mathbb{X}$ is a complete Souslin e.m.t.m. space. A function $f \in$ $L^{p}(X, \mathfrak{m})$ admits a $\mathcal{T}_{q}$-weak upper gradient $g \in L^{p}(X, \mathfrak{m})$ if and only if there is a Borel representative $\tilde{f}: X \rightarrow \mathbb{R}$ with $\mathfrak{m}(\{\tilde{f} \neq f\})=0$ which belongs to the Newtonian space $N^{1, p}(\mathbb{X})$. Equivalently, $\tilde{f}$ is absolutely continuous along $\operatorname{Mod}_{p}$-a.e. arc and g satisfies (10.41) $\operatorname{Mod}_{p}$-a.e. In particular, the class of $\mathcal{T}_{q}$-w.u.g. for $f$ coincides with the class of $N^{1, p}$-w.u.g. for a suitable Borel representative $\tilde{f}$ of $f$.

\subsection{Minimal $\mathcal{T}_{q}$-weak upper gradient and the Sobolev space $W^{1, p}\left(\mathbb{X}, \mathcal{T}_{q}\right)$.}

We want now to characterize the minimal $\mathcal{T}_{q}$-w.u.g. of a function and the corresponding notion of Sobolev space. We first prove two important properties. The first one directly involves the characterization of Theorem 10.14 .

Proposition 10.21 (Locality). Let $f: X \rightarrow \mathbb{R}$ be $\mathfrak{m}$-measurable and let $g_{1}, g_{2}$ be weak upper gradients of $f$ w.r.t. $\mathcal{T}_{q}$. Then $\min \left\{g_{1}, g_{2}\right\}$ is a $\mathcal{T}_{q}$-weak upper gradient of $f$.

Proof. We know from Theorem 10.14 that $f$ is Sobolev along $\mathcal{T}_{q}$-almost every arc. Then, the claim is a direct consequence of Remark 10.15 and (10.16).

Another important property of weak upper gradients is their stability w.r.t. weak $L^{p}$ convergence. 
Theorem 10.22 (Stability w.r.t. weak convergence). Assume that $f_{n} \in L^{1}(X, \mathfrak{m})$ and that $g_{n} \in$ $L^{p}(X, \mathfrak{m})$ are $\mathcal{T}_{q}$-weak upper gradients of $f_{n}$. If $f_{n} \rightarrow f$ weakly in $L^{1}(X, \mathfrak{m})$ and $g_{n} \rightarrow g$ weakly in $L^{p}(X, \mathfrak{m})$ as $n \rightarrow \infty$, then $f$ is Sobolev along $\mathcal{T}_{q}$-a.e. arc and $g$ is a $\mathcal{T}_{q}$-weak upper gradient of $f$.

Proof. We can apply Lemma 10.8: we fix a test plan $\pi \in \mathcal{T}_{q}^{*}$ and set $h_{0}, h_{1} \in L^{\infty}(X, \mathfrak{m}), h \in$ $L^{q}(X, \mathfrak{m})$ such that

$$
\left(\mathrm{e}_{0}\right)_{\sharp} \boldsymbol{\pi}=\pi_{0}=h_{0} \mathfrak{m}, \quad\left(\mathrm{e}_{1}\right)_{\sharp} \boldsymbol{\pi}=\pi_{1}=h_{1} \mathfrak{m}, \quad \mu_{\boldsymbol{\pi}}=h \mathfrak{m} .
$$

Since $g_{n}$ is a $\mathcal{T}_{q}$-weak upper gradient for $f_{n}$ we know that

$$
\int_{X} f_{n} \mathrm{~d}\left(\pi_{1}-\pi_{0}\right)=\int_{X} f_{n}\left(h_{1}-h_{0}\right) \mathrm{d} \mathfrak{m} \leq \int_{X} g_{n} \mathrm{~d} \mu_{\boldsymbol{\pi}}=\int_{X} g_{n} h \mathrm{~d} \mathfrak{m} .
$$

Passing to the limit by weak convergence in $L^{1}$ and $L^{p}$ we immediately get

$$
\int_{X} f \mathrm{~d}\left(\pi_{1}-\pi_{0}\right) \leq \int_{X} g \mathrm{~d} \mu_{\pi}
$$

Since $\pi \in \mathcal{T}_{q}$ is arbitrary, we conclude.

We can now formalize the notion of $\mathcal{T}_{q}$-minimal weak upper gradient. For the sake of simplicity, here we will consider only the case of functions with $\mathcal{T}_{q}$-w.u.g. in $L^{p}(X, \mathfrak{m})$.

Definition 10.23 (Minimal $\mathcal{T}_{q}$-weak upper gradient). Let $f \in L^{1}(X, \mathfrak{m})$ be a $\mathfrak{m}$-measurable function with a $\mathcal{T}_{q}$-weak upper gradient in $L^{p}(X, \mathfrak{m})$. The $\mathcal{T}_{q}$-minimal weak upper gradient $|\mathrm{D} f|_{w, \mathcal{T}_{q}}$ of $f$ is the $\mathcal{T}_{q}$-weak upper gradient characterized, up to $\mathfrak{m}$-negligible sets, by the property

$$
|\mathrm{D} f|_{w, \mathcal{T}_{q}} \leq g \quad \mathfrak{m} \text {-a.e. in } X \text {, for every } \mathcal{T}_{q} \text {-weak upper gradient } g \text { of } f \text {. }
$$

Uniqueness of the minimal weak upper gradient is obvious. For existence, let us consider a minimizing sequence $\left(g_{n}\right)_{n \in \mathbb{N}} \subset L^{p}(X, \mathfrak{m})$ for the problem

$$
\inf \left\{\int_{X} g^{p} \mathrm{dm}: g \text { is a } \mathcal{T}_{q} \text {-weak upper gradient of } f\right\} .
$$

We immediately see, thanks to Theorem 10.22 , that we can assume with no loss of generality that $g_{n} \rightarrow g_{\infty}$ in $L^{p}(X, \mathfrak{m})$ and $g_{\infty}$ is the $\mathcal{T}_{q}$-weak upper gradient of $f$ of minimal $L^{p}$-norm. This minimality, in conjunction with Proposition 10.21, gives (10.43) for $|\mathrm{D} f|_{w, \mathcal{T}_{q}}:=g_{\infty}$.

Definition 10.24 (The weak $\left(\mathcal{T}_{q}, p\right)$-energy and the Sobolev space $W^{1, p}\left(\mathbb{X}, \mathcal{T}_{q}\right)$ ). Let $f \in L^{1}(X, \mathfrak{m})$ with a $\mathcal{T}_{q}$-weak upper gradient $g \in L^{p}(X, \mathfrak{m})$. The weak $\left(\mathcal{T}_{q}, p\right)$-energy of $f$ is defined by

$$
w C E_{p, \mathcal{T}_{q}}(f)={ } \mathrm{wE}_{p}(f):=\int_{X}|\mathrm{D} f|_{w, \mathcal{T}_{q}}^{p} \mathrm{dm} .
$$

If moreover $f \in L^{p}(X, \mathfrak{m})$ we say that $f$ belongs to the space $W^{1, p}\left(\mathbb{X}, \mathcal{T}_{q}\right) . W^{1, p}\left(\mathbb{X}, \mathcal{T}_{q}\right)$ is a Banach space endowed with the norm

$$
\|f\|_{W^{1, p}\left(\mathbb{X}, \mathcal{T}_{q}\right)}^{p}:=\int_{X}\left(f^{p}+|\mathrm{D} f|_{w, \mathcal{T}_{q}}^{p}\right) \mathrm{d} \mathfrak{m}=\|f\|_{L^{p}(X, \mathfrak{m})}^{p}+\mathrm{wCE}_{p, \mathcal{T}_{q}}(f) .
$$


Remark 10.25 (The $\mathcal{T}_{q}$-notation). Even if we will mainly use minimal w.u.g. induced by $\mathcal{T}_{q}$-test plan, we will keep the explicit occurrence of $\mathcal{T}_{q}$ in the notation $|\mathrm{D} f|_{w, \mathcal{T}_{q}}$ and $\mathrm{wCE}_{p, \mathcal{T}_{q}}$ in order to distinguish these notions from other definitions of weak upper gradients based on different class of test plan (also on parametric arcs), which usually share the symbol $|\mathrm{D} f|_{w}$. We will use the shorter notation wCE $\mathrm{E}_{p}$ only when no risk of confusion will be possible.

By using the same approach, the construction of the minimal $p$-weak upper gradient $|\mathrm{D} f|_{w, N^{1, p}}$ can also be performed for functions in the Newtonian space $N^{1, p}(\mathbb{X})$, and gives raise to the (semi)norm

$$
\|f\|_{N^{1, p}(\mathbb{X})}^{p}:=\int_{X}\left(|f|^{p}+|\mathrm{D} f|_{w, N^{1, p}}^{p}\right) \mathrm{d} \mathfrak{m} .
$$

Taking Corollary 10.20 into account we easily have:

Corollary 10.26 (The link with the Newtonian space $N^{1, p}(\mathbb{X})$ ). Let us suppose that $\mathbb{X}$ is a complete Souslin e.m.t.m. space. (The Lebesgue equivalence class of) every function $f \in N^{1, p}(\mathbb{X})$ belongs to $W^{1, p}\left(\mathbb{X}, \mathcal{T}_{q}\right)$. Conversely, every function $f \in W^{1, p}\left(\mathbb{X}, \mathcal{T}_{q}\right)$ has an equivalent representative $\tilde{f}$ in $N^{1, p}(\mathbb{X})$ with

$$
|\mathrm{D} f|_{w, \mathcal{T}_{q}}=|\mathrm{D} \tilde{f}|_{w, N^{1, p}} \quad \text { a.e., } \quad\|f\|_{W^{1, p}\left(\mathbb{X}, \mathcal{T}_{q}\right)}=\|\tilde{f}\|_{N^{1, p}(\mathbb{X})} .
$$

It is easy to check using (10.10) and Theorem 10.22 that the weak Cheeger energy ${ } \mathrm{CE}_{p, \mathcal{T}_{q}}$ is a convex, $p$-homogeneous, weakly lower-semicontinuous functional in $L^{1}(X, \mathfrak{m})$. It is also easy to state a first comparison with the strong Cheeger energy $\mathrm{CE}_{p}$ (the corresponding inequalities for $\mathrm{CE}_{p, \mathscr{A}}$ and $|\mathrm{D} f|_{\star, \mathscr{A}}$ follow trivially by (6.40) and (6.41)).

Lemma 10.27. Every function $f \in H^{1, p}(\mathbb{X})$ belongs to $W^{1, p}\left(\mathbb{X}, \mathcal{T}_{q}\right)$ and

$$
\mathrm{CE}_{p}(f) \geq \mathrm{wCE}_{p, \mathcal{T}_{q}}(f), \quad|\mathrm{D} f|_{\star} \geq|\mathrm{D} f|_{w, \mathcal{T}_{q}} \quad \mathfrak{m} \text {-a.e. in } X .
$$

Proof. We already notice that for a Lipschitz function $f \in \operatorname{Lip}_{b}(X, \tau, \mathrm{d}) \operatorname{lip} f$ is a $\mathcal{T}_{q}$-w.u.g. so that

$$
\mathrm{pCE}_{p}(f) \geq \mathrm{wCE}_{p, \mathcal{T}_{q}}(f) .
$$

It is then sufficient to take an optimal sequence $f_{n} \in \operatorname{Lip}_{b}(X, \tau, \mathrm{d})$ as in (5.9) and to apply the stability Theorem 10.22.

Proposition 10.28 (Chain rule for minimal weak upper gradients). If $f \in L^{1}(X, \mathfrak{m})$ has a $\mathcal{T}_{q}$-weak upper gradient in $L^{p}(X, \mathfrak{m})$, the following properties hold:

(a) for any $\mathscr{L}^{1}$-negligible Borel set $N \subset \mathbb{R}$ it holds $|\mathrm{D} f|_{w, \mathcal{T}_{q}}=0 \mathrm{~m}$-a.e. on $f^{-1}(N)$.

(b) $|\mathrm{D} \phi(f)|_{w, \mathcal{T}_{q}}=\phi^{\prime}(f)|\mathrm{D} f|_{w, \mathcal{T}_{q}} \mathfrak{m}$-a.e. in $X$, with the convention $0 \cdot \infty=0$, for any nondecreasing function $\phi$, Lipschitz on an interval containing the image of $f$.

Proof. We use the equivalent formulation of Remark 10.15 and the well-known fact that both (a) and (b) are true when $X=\mathbb{R}$ endowed with Euclidean distance and Lebesgue measure and $f$ is absolutely continuous. We can prove (a) setting

$$
G(x):= \begin{cases}|\mathrm{D} f|_{w, \mathcal{T}_{q}}(x) & \text { if } f(x) \in \mathbb{R} \backslash N \\ 0 & \text { if } f(x) \in N\end{cases}
$$

and noticing the validity of (a) for real-valued absolutely continuous maps gives that $G$ is $\mathcal{T}_{q}$-weak upper gradient of $f$. Then, the minimality of $|\mathrm{D} f|_{w, \mathcal{T}_{q}}$ gives $|\mathrm{D} f|_{w, \mathcal{T}_{q}} \leq G$ m-a.e. in $X$.

By a similar argument based on (10.16) we can prove that $|\mathrm{D} \phi(f)|_{w, \mathcal{T}_{q}} \leq \phi^{\prime}(f)|\mathrm{D} f|_{w, \mathcal{T}_{q}} \mathfrak{m}$-a.e. in $X$. Then, the same subadditivity argument of Theorem 5.12(c) provides the equality $\mathfrak{m}$-a.e. in $X$. 


\subsection{Invariance properties of weak Sobolev spaces}

In this section we will state a few useful results on the behaviour of weak Sobolev spaces with respect to some basic operations.

\section{Restriction}

Lemma 10.29. Let $\mathbb{X}=(X, \tau, \mathrm{d}, \mathfrak{m})$ be an e.m.t.m. space and let $Y \subset X$ be a $\mathrm{d}$-closed set such that $\mathfrak{m}(X \backslash Y)=0$. Then every dynamic plan $\pi \in \mathcal{B}_{q}$ is concentrated on $\operatorname{RA}(Y)$.

Proof. Let $\boldsymbol{\pi} \in \mathcal{M}_{+}(\operatorname{RA}(X))$ with $\operatorname{Bar}_{q}(\boldsymbol{\pi})<\infty$. Setting $Z:=X \backslash Y$ we have

$$
\iint_{\gamma} \chi_{Z} \mathrm{~d} \boldsymbol{\pi}(\gamma)=\int_{X} \chi_{Z} \mathrm{~d} \mu_{\boldsymbol{\pi}}=0
$$

since $\boldsymbol{\mu}_{\boldsymbol{\pi}} \ll \mathfrak{m}$ and $\mathfrak{m}(Z)=0$. We deduce that for $\boldsymbol{\pi}$-a.e. $\gamma \int_{\gamma} \chi_{Z}=0$, i.e. $\mathscr{L}^{1}\left(\left\{t \in[0,1]: R_{\gamma}(t) \in\right.\right.$ $Z\})=0$. Since $Z$ is d-open, it follows that $R_{\gamma}([0,1]) \subset Y$, i.e. $\pi$-a.e. $\gamma$ belongs to $\operatorname{RA}(Y)$.

Corollary 10.30 (Invariance of $W^{1, p}$ by restriction). Let $Y \subset X$ be a $\mathrm{d}$-closed set such that $\mathfrak{m}(X \backslash$ $Y)=0$. Setting $\mathbb{Y}:=(Y, \tau, \mathrm{d}, \mathfrak{m})$, we have $W^{1, p}\left(\mathbb{X}, \mathcal{T}_{q}\right)=W^{1, p}\left(\mathbb{Y} ; \mathcal{T}_{q}\right)$ and for every Sobolev function $f$ the minimal $\mathcal{T}_{q}(\mathbb{X})$-weak upper gradient coincides with the minimal $\mathcal{T}_{q}(\mathbb{Y})$-weak upper gradient.

\section{Measure preserving isometric embeddings}

Let $\mathbb{X}=(X, \tau, \mathrm{d}, \mathfrak{m})$ and $\mathbb{X}^{\prime}=\left(X^{\prime}, \tau^{\prime}, \mathrm{d}^{\prime}, \mathfrak{m}^{\prime}\right)$ be e.m.t.m. spaces and let suppose that $\iota: X \rightarrow X^{\prime}$ is a measure-preserving embedding according to Definition 2.28. We will call $\mathcal{T}_{q}^{\prime}=\mathcal{T}_{q}\left(\mathbb{X}^{\prime}\right)$ the class of nonparametric test plans in $\mathcal{M}_{+}\left(\mathrm{RA}\left(X^{\prime}\right)\right)$.

Starting from $\iota$ we can define a continuous injective map $J: \mathrm{C}([0,1] ; X) \rightarrow \mathrm{C}\left([0,1] ; X^{\prime}\right)$ by setting $J(\gamma):=\iota \circ \gamma$. Thanks to the isometric property of $\iota, J\left(\operatorname{BVC}([0,1] ; X) \subset \operatorname{BVC}\left([0,1] ; X^{\prime}\right)\right.$ and clearly $J$ is preserves equivalence classes of curves, so that $J$ induces a continuous injective map from $\mathrm{RA}(X)$ to $\mathrm{RA}\left(X^{\prime}\right)$ satisfying

$$
\int_{J \gamma} f^{\prime}=\int_{\gamma} f^{\prime} \circ \iota, \quad \ell(J \gamma)=\ell(\gamma) \quad \text { for every } \gamma \in \mathrm{RA}(X), f^{\prime} \in \mathrm{B}_{b}\left(X^{\prime}\right) .
$$

It is interesting to notice that

$$
\iota \text { is surjective } \Rightarrow J \text { is surjective. }
$$

In fact, given an $\operatorname{arc} \gamma^{\prime} \in \mathrm{RA}\left(X^{\prime}\right)$ we can consider the curve $R:=\iota^{-1} \circ R_{\gamma^{\prime}}$ which satisfies

$$
\mathrm{d}(R(s), R(t))=\mathrm{d}^{\prime}\left(R_{\gamma^{\prime}}(s), R_{\gamma^{\prime}}(t)\right)=\ell\left(\gamma^{\prime}\right)|t-s|
$$

so that $R \in \operatorname{Lip}_{c}([0,1] ;(X, \mathrm{~d})) \subset \operatorname{BVC}([0,1] ;(X, \mathrm{~d}))$ and $\gamma=\mathrm{q}(R) \in \operatorname{RA}(X)$ with $J \gamma=\gamma^{\prime}$.

Lemma 10.31. For every dynamic plan $\boldsymbol{\pi} \in \mathcal{M}_{+}(\mathrm{RA}(X))$ the push forward $\boldsymbol{\pi}^{\prime}:=J_{\sharp} \boldsymbol{\pi}$ is a dynamic plan in $\mathcal{M}_{+}\left(\mathrm{RA}\left(X^{\prime}\right)\right)$ satisfying

$$
\mu_{\boldsymbol{\pi}^{\prime}}=\iota_{\sharp} \mu_{\boldsymbol{\pi}}, \quad \int \ell(\gamma) \mathrm{d} \boldsymbol{\pi}(\gamma)=\int \ell\left(\gamma^{\prime}\right) \mathrm{d} \boldsymbol{\pi}^{\prime}\left(\gamma^{\prime}\right), \quad\left(\mathrm{e}_{i}\right)_{\sharp} \boldsymbol{\pi}^{\prime}=\iota_{\sharp}\left(\left(\mathrm{e}_{i}\right)_{\sharp} \boldsymbol{\pi}\right) \quad i=0,1 .
$$

In particular

$$
\operatorname{Bar}_{q}\left(\boldsymbol{\pi}^{\prime}\right)=\operatorname{Bar}_{q}(\boldsymbol{\pi})
$$

and $\boldsymbol{\pi}^{\prime}$ belongs to $\mathcal{T}_{q}^{\prime}$ if and only if $\boldsymbol{\pi}$ belongs to $\mathcal{T}_{q}$. 
Proof. For every nonnegative $f^{\prime} \in \mathrm{C}_{b}\left(X^{\prime}\right)$ we have by (10.50)

$$
\int f^{\prime} \mathrm{d} \mu_{\boldsymbol{\pi}^{\prime}}=\iint_{\gamma^{\prime}} f^{\prime} \mathrm{d} \boldsymbol{\pi}^{\prime}\left(\gamma^{\prime}\right)=\iint_{J \gamma} f^{\prime} \mathrm{d} \boldsymbol{\pi}(\gamma) \stackrel{(10.50)}{=} \iint_{\gamma} f^{\prime} \circ \iota \mathrm{d} \boldsymbol{\pi}(\gamma)=\int f^{\prime} \circ \iota \mathrm{d} \mu_{\boldsymbol{\pi}},
$$

which shows the first identity of (10.52). The second follows easily by choosing $f \equiv 1$ and the third identity is a consequence of the relation $\mathrm{e}_{i} \circ J=\iota \circ \mathrm{e}_{i}, i=0,1$. Since $\iota$ is injective, (10.53) is a consequence of the general properties of relative entropy functionals

$$
\operatorname{Bar}_{q}^{q}\left(\boldsymbol{\pi}^{\prime}\right)=\mathscr{L}^{q}\left(\mu_{\boldsymbol{\pi}^{\prime}} \mid \mathfrak{m}^{\prime}\right)=\mathscr{L}^{q}\left(\iota_{\sharp} \mu_{\boldsymbol{\pi}^{\prime}} \mid \iota_{\sharp} \mathfrak{m}^{\prime}\right)=\mathscr{L}^{q}\left(\mu_{\boldsymbol{\pi}} \mid \mathfrak{m}\right)=\operatorname{Bar}_{q}^{q}(\boldsymbol{\pi}) .
$$

A similar argument shows that $\mathscr{L}^{q}\left(\left(\mathrm{e}_{i}\right)_{\sharp} \boldsymbol{\pi}^{\prime} \mid \mathfrak{m}^{\prime}\right)=\mathscr{L}^{q}\left(\left(\mathrm{e}_{i}\right)_{\sharp} \boldsymbol{\pi} \mid \mathfrak{m}\right)$.

A simple but important application of the previous two Lemma yields the following result.

Theorem 10.32. Let $\iota: X \rightarrow X^{\prime}$ be a measure-preserving isometric imbedding of $\mathbb{X}$ into $\mathbb{X}^{\prime}$. For every $f^{\prime} \in W^{1, p}\left(\mathbb{X}^{\prime}, \mathcal{T}_{q}^{\prime}\right)$ the function $f:=\iota^{*} f^{\prime}$ belongs to $W^{1, p}\left(\mathbb{X}, \mathcal{T}_{q}\right)$ and

$$
|\mathrm{D} f|_{w, \mathcal{T}_{q}} \leq \iota^{*}\left(\left|\mathrm{D} f^{\prime}\right|_{w, \mathcal{T}_{q}^{\prime}}\right) \quad \mathfrak{m} \text {-a.e. in } X .
$$

If moreover $\iota$ is surjective or $(X, \mathrm{~d})$ is complete then $\iota^{*}$ is an isomorphism between $W^{1, p}\left(\mathbb{X}^{\prime}, \mathcal{T}_{q}^{\prime}\right)$ and $W^{1, p}\left(\mathbb{X}, \mathcal{T}_{q}\right)$ whose inverse is $\iota_{*}$ and

$$
|\mathrm{D} f|_{w, \mathcal{T}_{q}}=\iota^{*}\left(\left|\mathrm{D} f^{\prime}\right|_{w, \mathcal{T}_{q}^{\prime}}\right) \quad \mathfrak{m} \text {-a.e. in } X .
$$

Proof. Let $g^{\prime} \in L^{p}\left(X^{\prime}, \mathfrak{m}^{\prime}\right)$ be a $\mathcal{T}_{q}^{\prime}$-weak upper gradient of $f^{\prime}$ in $X^{\prime}$ and let $\boldsymbol{\pi} \in \mathcal{T}_{q}$. We want to show that $g:=\iota^{*} g^{\prime} \in L^{p}(X, \mathfrak{m})$ is a $\mathcal{T}_{q}$-weak upper gradient for $f:$ we use the equivalent characterization (10.12) of Lemma 10.8.

For every plan $\boldsymbol{\pi} \in \mathcal{T}_{q}$ Lemma 10.31 shows that $\boldsymbol{\pi}^{\prime}:=J_{\sharp} \boldsymbol{\pi} \in \mathcal{T}_{q}^{\prime}$ with $\mu_{\boldsymbol{\pi}^{\prime}}=\iota_{\sharp} \mu_{\boldsymbol{\pi}}$ and $\left(\mathrm{e}_{i}\right)_{\sharp} \boldsymbol{\pi}^{\prime}=$ $\iota_{\sharp}\left(\mathrm{e}_{i}\right)_{\sharp} \boldsymbol{\pi}$. We then obtain

$$
\begin{aligned}
\int_{X} f \mathrm{~d} \pi_{0}-\int_{X} f \mathrm{~d} \pi_{1} & =\int_{X} f^{\prime} \circ \iota \mathrm{d} \pi_{0}-\int_{X} f^{\prime} \circ \iota \mathrm{d} \pi_{1}=\int_{X^{\prime}} f^{\prime} \mathrm{d}\left(\iota_{\sharp} \pi_{0}\right)-\int_{X^{\prime}} f^{\prime} \mathrm{d}\left(\iota_{\sharp} \pi_{1}\right) \\
& =\int_{X^{\prime}} f^{\prime} \mathrm{d} \pi_{0}^{\prime}-\int_{X^{\prime}} f^{\prime} \mathrm{d} \pi_{1}^{\prime} \leq \int_{X^{\prime}} g^{\prime} \mathrm{d} \mu_{\pi^{\prime}} \\
& =\int_{X} g^{\prime} \mathrm{d}\left(\iota_{\sharp} \mu_{\boldsymbol{\pi}}\right)=\int g^{\prime} \circ \iota \mathrm{d} \mu_{\boldsymbol{\pi}}=\int g \mathrm{~d} \mu_{\boldsymbol{\pi}} .
\end{aligned}
$$

If $\iota$ is surjective, then $J$ is surjective by (10.51), so that the very same argument shows that any $\mathcal{T}_{q^{-}}$ weak upper gradient for $f \in L^{p}(X, \mathfrak{m})$ yields a weak $\mathcal{T}_{q}^{\prime}$ weak upper gradient $g^{\prime}:=\iota_{*} g$ for $\iota_{*} f$ in $L^{p}\left(X^{\prime}, \mathfrak{m}^{\prime}\right)$ with $\left\|g^{\prime}\right\|_{L^{q}\left(X^{\prime}, \mathfrak{m}^{\prime}\right)}=\|g\|_{L^{q}(X, \mathfrak{m})}$ thus showing (10.56).

When $(X, \mathrm{~d})$ is complete then $\left(\iota(X), \mathrm{d}^{\prime}\right)$ is complete (and therefore $\mathrm{d}^{\prime}$-closed) in $X^{\prime}$, so that by Corollary $10.30 W^{1, p}\left(\iota(X), \tau^{\prime}, \mathrm{d}^{\prime}, \mathfrak{m}^{\prime} ; \mathcal{T}_{q}^{\prime}\right)=W^{1, p}\left(X^{\prime}, \tau^{\prime}, \mathrm{d}^{\prime}, \mathfrak{m}^{\prime} ; \mathfrak{T}_{q}^{\prime}\right)$ with equality of minimal $\mathcal{T}_{q^{-}}^{\prime}$ weak upper gradients. On the other hand, $\iota: X \rightarrow \iota(X)$ is a measure preserving surjective embedding and we can apply the previous statement.

\section{Length distances and conformal invariance}

We refer to the definitions and notation of Section 4. 
Lemma 10.33. Let $\mathbb{X}=(X, \tau, \mathrm{d}, \mathfrak{m})$ be an e.m.t.m. space and let $\delta: X \times X \rightarrow[0,+\infty]$ an extended distance such that

$$
(X, \tau, \delta) \text { is an extended metric-topological space, } \quad \mathrm{d} \leq \delta \leq \mathrm{d}_{\ell} \quad \text { in } X \times X .
$$

Then $W^{1, p}\left(X, \tau, \mathrm{d}, \mathfrak{m} ; \mathcal{T}_{q}\right)=W^{1, p}\left(X, \tau, \delta, \mathfrak{m} ; \mathcal{T}_{q}\right)$ and the corresponding minimal weak $\mathcal{T}_{q}$-upper gradients coincide.

Proof. We know that the class of rectifiable arcs $\mathrm{RA}(X, \mathrm{~d})$ and $\mathrm{RA}(X, \delta)$ coincide, since $\mathrm{d}_{\ell}=\delta_{\ell}$, with the same length. Therefore, the corresponding classes of dynamic plans in $\mathcal{T}_{q}$ coincide.

By the previous result, we can always replace $\mathrm{d}$ with $\mathrm{d}_{\ell}^{\prime}, \mathrm{d}_{\ell}^{\prime \prime}$ or $\mathrm{d}_{\ell}^{\prime \prime \prime}$ in the definition of the Sobolev spaces. We can also use $\mathrm{d}_{\ell}$ whenever $\mathrm{d}_{\ell}$ is $\tau$-continuous or when $(X, \tau)$ is compact.

Remark 10.34. The (easy) proof of the previous Lemma shows that the definition of the Sobolev space $W^{1, p}\left(X, \tau, \delta, \mathfrak{m} ; \mathcal{T}_{q}\right)$ can be extended to a slightly more general setting: in fact, the condition that $(X, \tau, \delta)$ is an e.t.m. space can be relaxed by asking that there exists an extended distance $\mathrm{d}$ : $X \times X \rightarrow[0,+\infty]$ such that

$$
(X, \tau, \mathrm{d}) \text { is an e.t.m. space and } \mathrm{d} \leq \delta \leq \mathrm{d}_{\ell} .
$$

We now discuss the case of a conformal distance $\mathrm{d}_{g}$ induced by a continuous function $g \in \mathrm{C}_{b}(X)$ with $\inf _{X} g>0$.

Proposition 10.35. Let $\mathbb{X}=(X, \tau, \mathrm{d}, \mathfrak{m})$ be an e.m.t.m. space, let $g \in \mathrm{C}_{b}(X)$ with $0<m_{g} \leq g \leq$ $M_{g}<\infty$, and let $\delta: X \times X \rightarrow[0,+\infty]$ be an extended distance such that

$$
\mathbb{X}^{\prime}=(X, \tau, \delta, \mathfrak{m}) \text { is an e.m.t.m. space, } \quad \mathrm{d}_{g}^{\prime} \leq \delta \leq \mathrm{d}_{g} \quad \text { in } X \times X .
$$

Then $\mathcal{T}_{q}(\mathbb{X})$ coincides with $\mathcal{T}_{q}^{\prime}:=\mathcal{T}_{q}\left(\mathbb{X}^{\prime}\right)$, a function $f \in L^{p}(X, \mathfrak{m})$ belongs to the Sobolev space $W^{1, p}\left(\mathbb{X}^{\prime} ; \mathcal{T}_{q}^{\prime}\right)$ if and only if $f \in W^{1, p}\left(\mathbb{X}, \mathcal{T}_{q}\right)$, and the corresponding minimal $\mathcal{T}_{q}$-weak upper gradients in $\mathbb{X}$ and in $\mathbb{X}^{\prime}$ (which we call $|\mathrm{D} f|_{w, \mathbb{X}}$ and $|\mathrm{D} f|_{w, \mathbb{X}^{\prime}}$ respectively) satisfy

$$
|\mathrm{D} f|_{w, \mathbb{X}}=g^{-1}|\mathrm{D} f|_{w, \mathbb{X}^{\prime}} .
$$

Proof. Denoting by $\int_{\gamma^{\prime}}$ the integration of a function $f$ along an $\operatorname{arc} \gamma$ with respect to the $\delta$ arc-length, we can easily check that

$$
\int_{\gamma^{\prime}} f=\int_{\gamma} g f
$$

It follows that if $\pi \in \mathcal{T}_{q}$

$$
\iint_{\gamma^{\prime}} f \mathrm{~d} \boldsymbol{\pi}(\gamma)=\iint_{\gamma} g f \mathrm{~d} \boldsymbol{\pi}(\gamma)=\int g f \mathrm{~d} \mu_{\boldsymbol{\pi}}=\int f \mathrm{~d} \mu_{\boldsymbol{\pi}}^{\prime}, \quad \mu_{\boldsymbol{\pi}}^{\prime}:=g \mu_{\boldsymbol{\pi}} .
$$

We deduce that

$$
m_{g}^{q} \mathscr{L}^{q}\left(\mu_{\boldsymbol{\pi}} \mid \mathfrak{m}\right) \leq \mathscr{L}^{q}\left(\boldsymbol{\mu}_{\boldsymbol{\pi}}^{\prime} \mid \mathfrak{m}\right) \leq M_{g}^{q} \mathscr{L}^{q}\left(\mu_{\boldsymbol{\pi}} \mid \mathfrak{m}\right)
$$

so that $\mathcal{T}_{q}=\mathcal{T}_{q}^{\prime}$. If $h \in L^{p}(X, \mathfrak{m})$ is a weak $\mathcal{T}_{q}$-upper gradient for $f$ in $\mathbb{X}$ we have

$$
\int f \mathrm{~d}\left(\pi_{0}-\pi_{1}\right) \leq \int h \mathrm{~d} \mu_{\boldsymbol{\pi}}=\int g\left(g^{-1} h\right) \mathrm{d} \mu_{\boldsymbol{\pi}}=\int g^{-1} h \mathrm{~d} \mu_{\boldsymbol{\pi}}^{\prime},
$$

which shows that $g^{-1} h$ is a $\mathcal{T}_{q}^{\prime}$-weak upper gradient for $f$ in $\mathbb{X}^{\prime}$. 


\subsection{The approach by parametric dynamic plans}

Let us give a brief account of the definition of the Sobolev space $W^{1, p}$ by parametric dynamic plans $[9,2]$, i.e. Radon measures on suitable subsets of $\mathrm{C}([0,1] ;(X, \tau))$, and their relations with the notions we introduced in the previous Sections.

We first define the space $\operatorname{AC}^{q}([0,1] ; X)$ as the collection of curves $\gamma \in \operatorname{BVC}([0,1] ;(X, \mathrm{~d}))$ such that $V_{\gamma}$ is absolutely continuous with derivative $|\dot{\gamma}|:=V_{\gamma}^{\prime} \in L^{q}(0,1)$. The $q$-energy of a curve $\gamma$ is defined by

$$
\mathrm{E}_{q}(\gamma):=\int_{0}^{1}|\dot{\gamma}|^{q} \mathrm{~d} t \quad \text { if } \gamma \in \mathrm{AC}^{q}([0,1] ; X) \quad \mathrm{E}_{q}(\gamma):=+\infty \text { otherwise }
$$

it defines a $\tau_{\mathrm{C}}$-lower semicontinuous map. It follows in particular that $\mathrm{AC}^{q}([0,1] ; X)$ is a $F_{\sigma}$ (thus Borel) subset of $\mathrm{C}([0,1] ;(X, \tau))$.

Recall that $\mathrm{e}_{t}: \mathrm{C}([0,1] ;(X, \tau)) \rightarrow X$ is the evaluation map $\mathrm{e}_{t}(\gamma)=\gamma(t)$.

Definition 10.36 (Parametric $q$-test plan, $[9,8])$. We denote by $\mathrm{T}_{q}$ the collection of all the Radon probability measures $\boldsymbol{\sigma}$ on $\mathrm{C}([0,1] ;(X, \tau))$ satisfying the following two properties:

(T1) there exists $M_{\sigma}>0$ such that

$$
\left(\mathrm{e}_{t}\right)_{\sharp} \boldsymbol{\sigma} \leq M_{\boldsymbol{\sigma}} \mathfrak{m} \quad \text { for every } t \in[0,1] .
$$

(T2) $\boldsymbol{\sigma}$ is concentrated on $\mathrm{AC}^{q}([0,1] ; X)$, i.e. $\boldsymbol{\sigma}\left(\mathrm{C}\left([0,1] ;(X, \tau) \backslash \mathrm{AC}^{q}([0,1] ; X)\right)=0\right.$;

We will call $\mathrm{T}_{q}^{*}$ the subset of dynamic plans in $\mathrm{T}_{q}$ with finite q-energy:

$$
\mathcal{E}_{q}(\boldsymbol{\sigma}):=\int \mathrm{E}_{q}(\gamma) \mathrm{d} \boldsymbol{\sigma}(\gamma)<\infty
$$

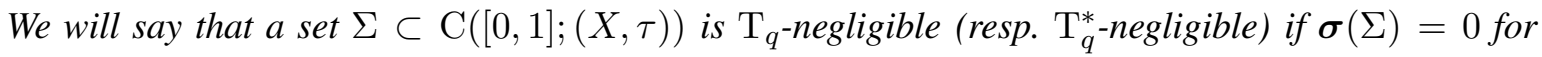
every $\boldsymbol{\sigma} \in \mathrm{T}_{q}$ (resp. $\boldsymbol{\sigma} \in \mathrm{T}_{q}^{*}$ ).

As usual, we will say that a property $P$ on curves of $\mathrm{C}([0,1] ;(X, \tau))$ holds $\mathrm{T}_{q}$-a.e. if the set where $P$ does not hold is $\mathrm{T}_{q}$-negligible.

Notice that if a set $\Sigma$ is $\mathrm{T}_{q}^{*}$-negligible then it is also $\mathrm{T}_{q}$ negligible: it is sufficient to approximate every plan $\sigma \in \mathrm{T}_{q}$ by an increasing sequence of plans satisfying (10.64).

Starting from the notion of $\mathrm{T}_{q}$-exceptional sets, we can introduce the corresponding definition of $\mathrm{T}_{q}$-weak upper gradient and Sobolev space.

Definition 10.37 ( $\mathrm{T}_{q}$-weak upper gradient). We say that a function $f \in L^{p}(X, \mathfrak{m})$ belongs to the Sobolev space $W^{1, p}\left(\mathbb{X}, \mathrm{T}_{q}\right)$ if there exists a function $g \in \mathcal{L}_{+}^{p}(X, \mathfrak{m})$ such that

$$
|f(\gamma(1))-f(\gamma(0))| \leq \int_{0}^{1} g(\gamma(t))|\dot{\gamma}|(t) \mathrm{d} t
$$

for $\mathrm{T}_{q}$-a.e. $\gamma \in \mathrm{AC}^{\mathrm{q}}([0,1] ; X)$. Every function $g$ with the stated property is called a $\mathrm{T}_{q}$-w.u.g. of $f$.

The properties of Sobolev functions in $W^{1, p}\left(\mathbb{X}, \mathrm{T}_{q}\right)$ can be studied by arguments similar to the ones we presented in $\S 10.1$ and $\S 10.3$, obtaining corresponding results adapted to the parametric $\mathrm{T}_{q}$-setting: we refer to $[9,8]$ for the precise statements and proofs. 
However, by adapting the arguments of [2], it is possible to prove directly that the notions of $\mathcal{T}_{q}$ and $\mathrm{T}_{q}$ weak upper gradient coincide, obtaining the equivalence of the corresponding Sobolev spaces $W^{1, p}\left(\mathbb{X}, \mathcal{T}_{q}\right)$ and $W^{1, p}\left(\mathbb{X}, \mathrm{T}_{q}\right)$.

First of all, it is not difficult to check that for every $f \in L^{p}(X, \mathfrak{m})$ and $g \in \mathcal{L}_{+}^{p}(X, \mathfrak{m})$

$$
g \text { is a } \mathcal{T}_{q} \text {-w.u.g. of } f \Rightarrow g \text { is a } \mathrm{T}_{q^{-} \text {-w.u.g. of } f},
$$

since for every parametric dynamic plan $\boldsymbol{\sigma} \in \mathrm{T}_{q}^{*}$ the corresponding nonparametric version $\boldsymbol{\pi}:=\mathfrak{q}_{\sharp} \boldsymbol{\sigma}$ belongs to $\mathcal{T}_{q}$; recall that we denoted by $\mathfrak{q}: \mathrm{C}([0,1] ;(X, \tau)) \rightarrow \mathrm{A}(X, \tau)$ the quotient map. In fact

$$
\left(\mathrm{e}_{i}\right)_{\sharp} \boldsymbol{\pi}=\left(\mathrm{e}_{i}\right)_{\sharp} \boldsymbol{\sigma} \leq M_{\boldsymbol{\sigma}} \mathfrak{m} \quad i=0,1,
$$

and for every bounded Borel function $f: X \rightarrow \mathbb{R}$

$$
\begin{aligned}
\iint_{\gamma} f \mathrm{~d} \boldsymbol{\pi}(\gamma) & =\iint_{\mathbf{q}(\eta)} f \mathrm{~d} \boldsymbol{\sigma}(\eta)=\iint_{0}^{1} f(\eta(t))|\dot{\eta}|(t) \mathrm{d} \boldsymbol{\sigma}(\eta) \\
& \leq \int\left(\int_{0}^{1} f^{p}(\eta(t)) \mathrm{d} t\right)^{1 / p} \mathrm{E}_{q}^{1 / q}(\eta) \mathrm{d} \boldsymbol{\sigma}(\eta) \leq \mathcal{E}_{p}^{1 / p}\left(\iint_{0}^{1} f^{p}(\eta(t)) \mathrm{d} t \mathrm{~d} \boldsymbol{\sigma}(\eta)\right)^{1 / p} \\
& \leq \mathcal{E}_{p}^{1 / p}\left(\int_{0}^{1} \int_{0}^{1} f^{p}\left(\mathrm{e}_{t}(\eta)\right) \mathrm{d} \boldsymbol{\sigma}(\eta) \mathrm{d} t\right)^{1 / p} \leq\left(M_{\boldsymbol{\sigma}} \mathcal{E}_{p}\right)^{1 / p}\left(\int_{X} f^{p} \mathrm{~d} \mathfrak{m}\right)^{1 / p} .
\end{aligned}
$$

We can deduce that for every $\Gamma \subset \mathrm{RA}(X)$

$$
\Gamma \text { is } \mathcal{T}_{q^{-} \text {-negligible }} \Rightarrow \mathfrak{q}^{-1}(\Gamma) \text { is } \mathrm{T}_{q} \text {-negligible }
$$

and therefore we get (10.66). In order to prove the converse property we introduce the notion of parametric barycenter of a Radon measure $\sigma \in \mathcal{M}_{+}(\mathrm{C}([0,1] ;(X, \tau)))$ : it is the image measure $\varrho_{\sigma}:=$ $\mathrm{e}_{\sharp}\left(\boldsymbol{\sigma} \otimes \mathscr{L}^{1}\right) \in \mathcal{M}_{+}(X)$, which satisfies

$$
\int_{X} f \mathrm{~d} \varrho_{\boldsymbol{\sigma}}=\iint_{0}^{1} f\left(\mathrm{e}_{t}(\gamma)\right) \mathrm{d} t \mathrm{~d} \boldsymbol{\sigma}(\gamma) \text { for every } f \in \mathrm{B}_{b}(X) .
$$

We say that $\boldsymbol{\sigma}$ has parametric barycenter in $L^{q}(X, \mathfrak{m})$ if $\varrho_{\boldsymbol{\sigma}}=h_{\boldsymbol{\sigma}} \mathfrak{m} \ll \mathfrak{m}$ for a density $h_{\boldsymbol{\sigma}} \in$ $L^{q}(X, \mathfrak{m})$.

The proof of the converse implication of (10.66) is based on the following two technical Lemmata.

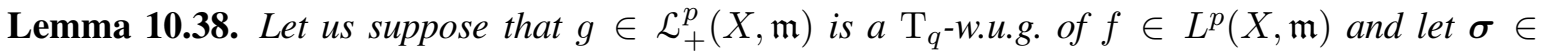
$\mathcal{M}_{+}(\mathrm{C}([0,1] ;(X, \tau)))$ be a dynamic plan satisfying (10.64) and

$$
\left(\mathrm{e}_{i}\right)_{\sharp} \boldsymbol{\sigma} \leq M \mathfrak{m} \ll \mathfrak{m} \quad i=0,1, \quad \varrho_{\boldsymbol{\sigma}} \leq M \mathfrak{m} \quad \text { for a constant } M>0 .
$$

Then (10.65) holds for $\sigma$-a.e. $\gamma$.

Proof. The argument is similar (but simpler) than the one used for the proof of Theorem 10.14. For $0 \leq r<s \leq 1$ we consider the Borel maps $D_{r}^{+}, D_{s}^{-}: \mathrm{C}([0,1], X) \times[0,1] \rightarrow \mathrm{C}([0,1] ;(X, \tau))$ defined by

$$
D^{+}[\gamma, r](t):=\gamma((r+t) \wedge 1), \quad D^{-}[\gamma, s](t):=\gamma((t-s) \vee 0)
$$

We then set $\lambda:=3 \mathscr{L}^{1}\llcorner(1 / 3,2 / 3)$ and which can also be characterized as

$$
\boldsymbol{\sigma}^{+}=\left(D^{+}\right)_{\sharp}(\boldsymbol{\sigma} \otimes \lambda), \quad \boldsymbol{\sigma}^{-}=\left(D^{-}\right)_{\sharp}(\boldsymbol{\sigma} \otimes \lambda) .
$$


We easily get for every $t \geq 2 / 3\left(\mathrm{e}_{t}\right)_{\sharp} \boldsymbol{\sigma}^{+}=\left(\mathrm{e}_{1}\right)_{\sharp} \boldsymbol{\sigma} \leq M \mathfrak{m}$, whereas for every $t \in[0,2 / 3)$ and every nonnegative Borel $f: X \rightarrow \mathbb{R}$

$$
\begin{aligned}
\int f\left(\mathrm{e}_{t}(\gamma)\right) \mathrm{d} \boldsymbol{\sigma}^{+}(\gamma) & =3 \int_{1 / 3}^{2 / 3}\left(\int f(\gamma((t+r) \wedge 1)) \mathrm{d} \boldsymbol{\sigma}(\gamma)\right) \mathrm{d} r \\
& =3 \int_{1 / 3}^{(1-t) \wedge 2 / 3}\left(\int f(\gamma(r+t)) \mathrm{d} \boldsymbol{\sigma}(\gamma)\right) \mathrm{d} r+3(1 / 3-t)_{+} \int f(\gamma(1)) \mathrm{d} \boldsymbol{\sigma}(\gamma) \\
& \leq 3 \int_{X} f \mathrm{~d} \varrho_{\boldsymbol{\sigma}}+\int_{X} f \mathrm{~d}\left(\mathrm{e}_{1}\right)_{\sharp} \boldsymbol{\sigma} \leq 4 M \int_{X} f \mathrm{~d} \mathfrak{m}
\end{aligned}
$$

so that $\left(\mathrm{e}_{t}\right)_{\sharp} \boldsymbol{\sigma}^{+} \leq 4 M \mathfrak{m}$ for every $t \in[0,1]$. An analogous calculation holds for $\boldsymbol{\sigma}^{-}$, so that both satisfy (3.28). Since $\mathrm{E}_{q}\left(D^{ \pm}(\gamma, r)\right) \leq \mathrm{E}_{q}(\gamma)$ for every $r \in[0,1]$ we also get (10.64). We deduce that $\boldsymbol{\sigma}^{+}, \boldsymbol{\sigma}^{-}$belong to $\mathrm{T}_{q}$ so that (10.65) holds for $\boldsymbol{\sigma}^{+}$and $\boldsymbol{\sigma}^{-}$-a.e. curve $\gamma$. Applying Fubini's theorem, we can find a common Borel and $\boldsymbol{\sigma}$-negligible set $N \subset \mathrm{C}([0,1] ;(X, \tau))$ such that (10.65) for every $\gamma \in \mathrm{AC}^{q}([0,1] ; X) \backslash N$

$$
\begin{aligned}
\mid f(\gamma(1-s))-f(\gamma(0) \mid & =\left|f\left(D^{-}[\gamma, s](1)\right)-f\left(D^{-}[\gamma, s](0)\right)\right| \\
& \leq \int_{0}^{1} g(\gamma((t-s) \vee 0))|\dot{\gamma}((t-s) \vee 0)| \mathrm{d} t \\
& =\int_{0}^{1-s} g(\gamma(t))|\dot{\gamma}|(t) \mathrm{d} t \quad \text { for a.e. } s \in(1 / 2,3 / 2)
\end{aligned}
$$

and similarly

$$
\begin{aligned}
\mid f(\gamma(1))-f(\gamma(r) \mid & =\left|f\left(D^{+}[\gamma, r](1)\right)-f\left(D^{+}[\gamma, r](0)\right)\right| \\
& \leq \int_{0}^{1} g(\gamma((t+r) \wedge 1))|\dot{\gamma}((t-s) \wedge 1)| \mathrm{d} t \\
& =\int_{r}^{1} g(\gamma(t))|\dot{\gamma}|(t) \mathrm{d} t \quad \text { for a.e. } r \in(1 / 2,3 / 2)
\end{aligned}
$$

For every $\gamma \in \operatorname{AC}^{q}([0,1] ; X)$ we can thus find a common value $r=1-s \in(1 / 2,3 / 2)$ such that the previous inequality hold, obtaining

$$
\mid f(\gamma(1))-f\left(\gamma(0)\left|\leq \int_{0}^{1-s} g(\gamma(t))\right| \dot{\gamma}\left|(t) \mathrm{d} t+\int_{r}^{1} g(\gamma(t))\right| \dot{\gamma}\left|(t) \mathrm{d} t=\int_{0}^{1} g(\gamma(t))\right| \dot{\gamma} \mid(t) \mathrm{d} t,\right.
$$

which yields (10.65).

The second Lemma is a reparametrization technique taken from [2, Theorem 8.5].

Lemma 10.39. For every nonparametric dynamic plan $\pi \in \mathcal{T}_{q}^{*}$ there exists a parametric dynamic plan $\sigma$ satisfying (10.69) such that

$$
\pi \ll \mathfrak{q}_{\sharp} \sigma .
$$

Combining Lemma 10.38 and 10.39 we obtain the following result, which shows the equivalence of the parametric and nonparametric approaches.

Corollary 10.40. For every $f \in L^{p}(X, \mathfrak{m})$ and $g \in \mathcal{L}_{+}^{p}(X, \mathfrak{m})$

$$
\text { gis a } \mathcal{T}_{q} \text {-w.u.g. of } f \quad \Longleftrightarrow \quad g \text { is a } \mathrm{T}_{q} \text {-w.u.g. of } f \text {. }
$$

In particular $W^{1, p}\left(\mathbb{X}, \mathcal{T}_{q}\right)=W^{1, p}\left(\mathbb{X}, \mathrm{T}_{q}\right)$. 
Proof. We have only to prove the converse implication of (10.66). Let $g \in \mathcal{L}_{+}^{p}(X, \mathfrak{m})$ be a $\mathrm{T}_{q^{-}}$ w.u.g. and let $\pi \in \mathcal{T}_{q}^{*}$. By Lemma 10.39 there exists a parametric dynamic plan $\sigma$ satisfying (10.69) such that $\boldsymbol{\pi} \ll \mathfrak{q}_{\sharp} \boldsymbol{\sigma}$. By Lemma 10.38 we know that (10.65) holds for $\boldsymbol{\sigma}$-a.e. curve, i.e.

$$
\left|f\left(\gamma_{1}\right)-f\left(\gamma_{0}\right)\right| \leq \int_{\gamma} g \quad \text { for } \mathfrak{q}_{\sharp} \boldsymbol{\sigma} \text {-a.e. } \gamma \in \mathrm{RA}(X) .
$$

Since $\boldsymbol{\pi} \ll \mathfrak{q}_{\sharp} \boldsymbol{\sigma}$ we deduce that (10.11) holds as well, so that we can apply Lemma 10.8 .

Remark 10.41. As for Cheeger's energy and the relaxed gradient, if no additional assumption on $(X, \tau, \mathrm{d}, \mathfrak{m})$ is made, it is well possible that the weak upper gradient is trivial. We will discuss this issue in the next Theorem 11.9.

\subsection{Notes}

$\S 10.1$ and $\S 10.3$ contain new definitions of weak upper gradient and weak Sobolev spaces based on the class of $\mathcal{T}_{q}$-weak upper gradients. It has some useful characteristics:

- it involves measures on nonparametric arcs; notice that the notion of upper gradient is inherently invariant w.r.t. parametrization, so arcs provide a natural setting;

- it is invariant w.r.t. modification on $\mathfrak{m}$-negligible sets;

- it seems quite close to the class $\mathcal{B}_{q}$ : one has only to add the control of the initial and final points of the $\operatorname{arcs}$

- the corresponding Modulus $\widetilde{\operatorname{Mod}}_{p}$ is strictly related to $\operatorname{Mod}_{p}$, so that via the selection of a "good representative" the Sobolev class $W^{1, p}\left(\mathbb{X}, \mathcal{T}_{q}\right)$ coincides with $N^{1, p}(\mathbb{X})$;

- it is directly connected with the dual of the Cheeger energy.

Of course, the study of the properties of the $\mathcal{T}_{q}$ w.u.g. retains many ideas of the corresponding analysis based on Radon measures on parametric curves $[9,8]$ as the stability, the Sobolev property along $\mathcal{T}_{q}^{*}$-a.e. arc, the chain rule. The rescaling technique of Theorem 10.14 has been also used in [2].

It is worth noticing that Corollary 10.26 could also be derived as a consequence of Theorem 11.7, as in [9, 8]. Here we followed the more direct approach of [2], which shows the closer link between $W^{1, p}$ and $N^{1, p}$.

$\S \mathbf{1 0 . 2}$ combines various methods introduced by [2]: apart from some topological aspects, Theorem 10.16 is a particular case of the identity between Modulus and Content at the level of collection of Radon measures, Proposition 10.17 uses the invariance of the Sobolev property by restriction and Theorem 10.18 is strongly inspired by [2, Theorem 10.3].

$\S 10.4$ contains natural invariance properties of weak Sobolev spaces: the most important one is (10.56) of Theorem 10.32, which will play a crucial role in the final part of the proof of the identification Theorem 11.7.

$\S 10.5$ contains a brief discussion of the equivalence between the nonparametric and parametric approaches to weak upper gradients and weak Sobolev spaces. It uses some of the arguments of [2] to show that the two approaches lead to equivalent definitions.

\section{Identification of Sobolev spaces}

In this Section we will prove the main identification Theorem for the Sobolev spaces $H^{1, p}(\mathbb{X}, \mathscr{A})$ and $W^{1, p}\left(\mathbb{X}, \mathcal{T}_{q}\right)$ when $(X, \mathrm{~d})$ is complete. As a first step we study a dual characterization of the weak $\left(\mathcal{T}_{q}, p\right)$-energy. 


\subsection{Dual Cheeger energies}

For every $\mu_{0}, \mu_{1} \in \mathcal{M}_{+}(X)$ we will introduce the (possibly empty) set

$$
\Pi\left(\mu_{0}, \mu_{1}\right):=\left\{\boldsymbol{\pi} \in \mathcal{M}_{+}(\operatorname{RA}(X)), \quad\left(\mathrm{e}_{i}\right)_{\sharp} \boldsymbol{\pi}=\mu_{i}\right\},
$$

and we define the cost functional

$$
\mathscr{D}_{q}\left(\mu_{0}, \mu_{1}\right):=\inf \left\{\operatorname{Bar}_{q}^{q}(\boldsymbol{\pi}): \boldsymbol{\pi} \in \Pi\left(\mu_{0}, \mu_{1}\right)\right\}, \quad \mathscr{D}_{q}\left(\mu_{0}, \mu_{1}\right)=+\infty \quad \text { if } \Pi\left(\mu_{0}, \mu_{1}\right)=\emptyset .
$$

Notice that $\Pi\left(\mu_{0}, \mu_{1}\right)$ is surely empty if $\mu_{0}(X) \neq \mu_{1}(X)$.

Let us check that if $\mathscr{D}_{q}\left(\mu_{0}, \mu_{1}\right)<+\infty$ and $(X, \mathrm{~d})$ is complete, then the infimum in (11.2) is attained. Notice that $\Pi\left(\mu_{0}, \mu_{1}\right)$ is a closed convex subset of $\mathcal{M}_{+}(\mathrm{A}(X))$.

Lemma 11.1. Let us suppose that $(X, \mathrm{~d})$ is complete. For every $\mu_{0}, \mu_{1} \in \mathcal{M}_{+}(X)$, if $\mathscr{D}_{q}\left(\mu_{0}, \mu_{1}\right)<$ $\infty$ then there exists a minimizer $\pi_{\min } \in \Pi\left(\mu_{0}, \mu_{1}\right)$ which realizes the infimum in (11.2). The set $\Pi_{o}\left(\mu_{0}, \mu_{1}\right)$ of optimal plans is a compact convex subset of $\mathcal{M}_{+}(\mathrm{RA}(X))$ and for every $\boldsymbol{\pi} \in$ $\Pi_{o}\left(\mu_{0}, \mu_{1}\right)$ the induced measure $\mu_{\boldsymbol{\pi}}$ is uniquely determined and is independent of the choice of the minimizer.

Proof. Let $\boldsymbol{\pi}^{\prime} \in \Pi\left(\mu_{0}, \mu_{1}\right)$ with $\operatorname{Bar}_{q} \boldsymbol{\pi}^{\prime}=E<\infty$ and define $\mathcal{K}:=\left\{\boldsymbol{\pi} \in \Pi\left(\mu_{0}, \mu_{1}\right): \operatorname{Bar}_{q}(\boldsymbol{\pi}) \leq\right.$ $E\}$. We can apply Lemma 8.5: for every $\boldsymbol{\pi} \in \Pi\left(\mu_{0}, \mu_{1}\right) \boldsymbol{\pi}(\mathrm{RA}(X))=\mu_{0}(X)$ so that condition (T1) is satisfied. Concerning (T2) it is sufficient to we use the tightness of $\mu_{0}$ to find compact sets $H_{\varepsilon} \subset X$ such that $\mu_{0}\left(X \backslash H_{\varepsilon}\right) \leq \varepsilon$; clearly

$$
\boldsymbol{\pi}\left(\left\{\gamma: \mathrm{e}(\gamma) \cap H_{\varepsilon}=\emptyset\right\}\right) \leq \boldsymbol{\pi}\left(\left\{\gamma: \mathrm{e}_{0}(\gamma) \cap H_{\varepsilon}=\emptyset\right\}\right)=\mu_{0}\left(X \backslash H_{\varepsilon}\right) \leq \varepsilon .
$$

Since the functional $\operatorname{Bar}_{q}$ is lower semicontinuous with respect to weak convergence, we conclude that the minimum is attained. The convexity and the compactness of $\Pi_{o}\left(\mu_{0}, \mu_{1}\right)$ are also immediate; the uniqueness of $\mu_{\boldsymbol{\pi}}$ when $\boldsymbol{\pi}$ varies in $\Pi_{o}\left(\mu_{0}, \mu_{1}\right)$ depends on the strict convexity of the $L^{q}(X, \mathfrak{m})$ norm and on the convexity of $\Pi_{o}\left(\mu_{0}, \mu_{1}\right)$.

We want to compare $\mathscr{D}_{q}$ with the dual of the pre-Cheeger energy:

$$
\frac{1}{q} \mathrm{pCE}_{p}^{*}(\mu):=\sup \left\{\int_{X} f \mathrm{~d} \mu-\frac{1}{p} \mathrm{pCE}_{p}(f): f \in \operatorname{Lip}_{b}(X, \tau, \mathrm{d})\right\}, \quad \mu=\mu_{0}-\mu_{1} \in \mathcal{M}(X) .
$$

Notice that by Lemma A.7 we have the equivalent representation

$$
\mathrm{pCE}_{p}^{*}(\mu)=\sup \left\{\int_{X} f \mathrm{~d} \mu: f \in \operatorname{Lip}_{b}(X, \tau, \mathrm{d}), \mathrm{pCE}_{p}(f) \leq 1\right\} .
$$

Whenever $\mu=h \mathfrak{m}$ with $h \in L^{q}(X, \mathfrak{m})$, we can also consider the dual of the Cheeger energy

$$
\frac{1}{q} \mathrm{CE}_{p}^{*}(h):=\sup \left\{\int_{X} f h \mathrm{~d} \mathfrak{m}-\frac{1}{p} \mathrm{CE}_{p}(f): f \in H^{1, p}(\mathbb{X})\right\},
$$

and of the weak $\left(\mathcal{T}_{q}, p\right)$-energy ${ } C_{C}$ (defined by a formula analogous to (11.5)) that we will denote by $w C E_{p}^{*}$. An obvious necessary condition for the finiteness of $\mathrm{pCE}_{p}^{*}$ and of $\mathrm{CE}_{p}^{*}$ is given by

$$
\mathrm{pCE}_{p}^{*}(\mu)<+\infty \Rightarrow \mu(X)=0 ; \quad \mathrm{CE}_{p}^{*}(h)<+\infty \quad \Rightarrow \quad \int_{X} h \mathrm{~d} \mathfrak{m}=0 .
$$

Since $\mathrm{wCE}_{p}(f) \leq \mathrm{CE}_{p}(f)$ for every $f \in L^{p}(X, \mathfrak{m})$ and $\mathrm{CE}_{p}(f) \leq \mathrm{pCE}_{p}(f)$ for every $f \in \operatorname{Lip}_{b}(X, \tau, \mathrm{d})$, it is clear that

$$
{ } \mathrm{CE}_{p}^{*}(h) \geq \mathrm{CE}_{p}^{*}(h) \geq \mathrm{pCE}_{p}^{*}(h \mathfrak{m}) \quad \text { for every } h \in L^{p}(X, \mathfrak{m}) .
$$


Lemma 11.2. For every $\mu_{0}, \mu_{1} \in \mathcal{M}_{+}(X)$ we have

$$
\mathscr{D}_{q}\left(\mu_{0}, \mu_{1}\right) \geq \mathrm{pCE}_{p}^{*}\left(\mu_{0}-\mu_{1}\right) .
$$

If moreover $\mu_{i}=h_{i} \mathfrak{m}$ with $h_{i} \in L^{p}(X, \mathfrak{m}), h_{i} \geq 0$, then

$$
\mathscr{D}_{q}\left(h_{0} \mathfrak{m}, h_{1} \mathfrak{m}\right) \geq \mathrm{wCE}_{p}^{*}(h) \quad h=h_{0}-h_{1}
$$

Proof. We observe that for every $\boldsymbol{\pi} \in \Pi\left(\mu_{0}, \mu_{1}\right)$ we have

$$
\frac{1}{q} \operatorname{Bar}_{q}^{q}(\boldsymbol{\pi})=\sup _{g \in \mathcal{L}_{+}^{p}(X, \mathfrak{m})} \iint_{\gamma} g \mathrm{~d} \boldsymbol{\pi}-\frac{1}{p} \int g^{p} \mathrm{~d} \mathfrak{m} .
$$

Restricting the supremum to the functions $g:=\operatorname{lip} f$ for some $f \in \operatorname{Lip}_{b}(X, \mathrm{~d}, \mathfrak{m})$ and observing that in this case for every $\gamma \in \mathrm{RA}(X)$,

$$
f\left(\gamma_{0}\right)-f\left(\gamma_{1}\right) \leq \int_{\gamma} g
$$

we get

$$
\begin{aligned}
& \iint_{\gamma} g \mathrm{~d} \boldsymbol{\pi}-\frac{1}{p} \int g^{p} \mathrm{~d} \mathfrak{m} \geq \int\left(f\left(\gamma_{0}\right)-f\left(\gamma_{1}\right)\right) \mathrm{d} \boldsymbol{\pi}-\frac{1}{p} \mathrm{pCE}_{p}(f) \\
& =\int_{X} f \mathrm{~d}\left(\mu_{0}-\mu_{1}\right)-\frac{1}{p} \mathrm{pCE}_{p}(f)
\end{aligned}
$$

so that (11.8) follows by taking the supremum w.r.t. $f$ and the infimum w.r.t. $\pi$.

When $\mu_{i}=h_{i} \mathfrak{m}$ with $h_{i} \in L^{p}(X, \mathfrak{m})$ nonnegative, any dynamic plan $\pi \in \Pi\left(\mu_{0}, \mu_{1}\right)$ with $\operatorname{Bar}_{q}(\boldsymbol{\pi})<\infty$ belongs to $\mathcal{T}_{q}$. Restricting the supremum of (11.10) to (the Borel representative of) functions $g=|\mathrm{D} f|_{w, \mathcal{T}_{q}}$ for some $f \in W^{1, p}\left(\mathbb{X}, \mathcal{T}_{q}\right)$ it follows that (11.11) holds for $\mathcal{T}_{q}$-a.e. curve, in particular for $\pi$-a.e. curve $\gamma$. We can then perform the same integration with respect to $\pi$ and obtain (11.9).

\section{2 $H=W$}

\section{The compact case}

Let us first consider the case when $(X, \tau)$ is compact. For every strictly positive function $g \in \mathrm{C}_{b}(X)$ (we will still use the notation $\mathrm{C}_{b}(X)$ even if the subscript ${ }_{b}$ is redundant, being $X$ compact) we will denote by $\mathrm{d}_{g}$ the conformal distance we studied in $\S 4.2$ and by $\mathrm{K}_{\mathrm{d}_{g}}$ the Kantorovich-Rubinstein distance induced by $\mathrm{d}_{g}$, see $\S 2.4$. Notice that $\left(X, \tau, \mathrm{d}_{g}\right)$ is a geodesic e.m.t. space thanks to Theorem 4.2 .

Theorem 11.3. Let us suppose that $(X, \tau)$ is compact; then for every $\mu_{0}, \mu_{1} \in \mathcal{M}_{+}(X)$ with $\mu_{0}(X)=$ $\mu_{1}(X)$ we have

$$
\mathscr{D}_{q}\left(\mu_{0}, \mu_{1}\right)=\sup \left\{\mathrm{K}_{\mathrm{d}_{g}}\left(\mu_{0}, \mu_{1}\right)-\frac{1}{p} \int_{X} g^{p} \mathrm{~d} \mathfrak{m}: g \in \mathrm{C}_{b}(X), g>0\right\} .
$$


Proof. Let us introduce the convex set

$$
\mathcal{C}:=\left\{\left(g, \psi_{0}, \psi_{1}\right) \in\left(\mathrm{C}_{b}(X)\right)^{3}: g(x)>0 \quad \text { for every } x \in X\right\}
$$

and the dual representation of the convex set $\Pi\left(\mu_{0}, \mu_{1}\right)$ given by two Lagrange multipliers $\psi_{0}, \psi_{1} \in$ $\mathrm{C}_{b}(X): \boldsymbol{\pi} \in \Pi\left(\mu_{0}, \mu_{1}\right)$ if and only if (here $\left.\gamma_{i}=\mathrm{e}_{i}(\gamma), i=0,1\right)$

$$
\sup _{\psi_{0}, \psi_{1} \in \mathrm{C}_{b}(X)} \int_{X} \psi_{0} \mathrm{~d} \mu_{0}-\int \psi_{0}\left(\gamma_{0}\right) \mathrm{d} \boldsymbol{\pi}(\gamma)-\left(\int_{X} \psi_{1} \mathrm{~d} \mu_{1}-\int \psi_{1}\left(\gamma_{1}\right) \mathrm{d} \boldsymbol{\pi}(\gamma)\right)<+\infty
$$

Notice that whenever the supremum in (11.14) is finite, it vanishes. We first observe that

$$
\frac{1}{q} \mathscr{D}_{q}\left(\mu_{0}, \mu_{1}\right)=\inf _{\boldsymbol{\pi} \in \mathcal{M}_{+}(\operatorname{RA}(X))} \sup _{\left(g, \psi_{0}, \psi_{1}\right) \in \mathcal{C}} \mathcal{L}\left(\left(g, \psi_{0}, \psi_{1}\right) ; \boldsymbol{\pi}\right)
$$

where the Lagrangian function $\mathcal{L}$ is given by

$$
\begin{aligned}
\mathcal{L}\left(\left(g, \psi_{0}, \psi_{1}\right) ; \boldsymbol{\pi}\right):=\int( & \left.\int_{\gamma} g+\psi_{1}\left(\gamma_{1}\right)-\psi_{0}\left(\gamma_{0}\right)\right) \mathrm{d} \boldsymbol{\pi}(\gamma) \\
& +\int_{X} \psi_{0} \mathrm{~d} \mu_{0}-\int_{X} \psi_{1} \mathrm{~d} \mu_{1}-\frac{1}{p} \int_{X} g^{p} \mathrm{~d} \mathfrak{m},
\end{aligned}
$$

and it is clearly convex w.r.t. $\boldsymbol{\pi}$ and concave w.r.t. $\left(g, \psi_{0}, \psi_{1}\right)$. We want to apply Von Neumann Theorem A.8 and to invert the order of inf and sup.

Selecting $g_{\star} \equiv 1, \psi_{1, \star} \equiv 1, \psi_{0, \star} \equiv 0$ we see that for every $C \geq 0$ the sublevel

$$
\mathcal{K}_{C}:=\left\{\boldsymbol{\pi} \in \operatorname{RA}(X): \mathcal{L}\left(\left(g_{\star}, \psi_{0, \star}, \psi_{1, \star}\right) ; \boldsymbol{\pi}\right) \leq C\right\}
$$

is not empty (it contains the null plan) and compact, since for every $\pi \in \mathcal{K}_{C}$ we have

$$
\boldsymbol{\pi}(\mathrm{RA}(X))+\int \ell(\gamma) \mathrm{d} \boldsymbol{\pi} \leq C+\frac{1}{p} \mathfrak{m}(X)+\mu_{1}(X),
$$

so that $\mathcal{K}_{C}$ is equi-tight, thanks to Theorem 3.13(g) (here we use the compactness of $(X, \tau)$ ).

We therforetherefore obtain

$$
\mathscr{D}_{q}\left(\mu_{0}, \mu_{1}\right)=\sup _{\left(g, \psi_{0}, \psi_{1}\right) \in \mathcal{C}} \inf _{\boldsymbol{\pi} \in \mathcal{M}_{+}(\operatorname{RA}(X))} \mathcal{L}\left(\left(g, \psi_{0}, \psi_{1}\right) ; \boldsymbol{\pi}\right)
$$

We can introduce the conformal (extended) distance generated by $g$

$$
\mathrm{d}_{g}\left(x_{0}, x_{1}\right):=\inf \left\{\int_{\gamma} g: \gamma \in \operatorname{RA}(X), \gamma_{0}=x_{0}, \gamma_{1}=x_{1}\right\}
$$

observing that if the triple $\left(g, \psi_{0}, \psi_{1}\right)$ does not belong to the subset of $\mathcal{C}$

$$
\Sigma:=\left\{\left(g, \psi_{0}, \psi_{1}\right) \in \mathrm{C}_{b}(X)^{3}: g>0, \psi_{0}\left(x_{0}\right)-\psi_{1}\left(x_{1}\right) \leq \mathrm{d}_{g}\left(x_{0}, x_{1}\right) \quad \text { for every } x_{0}, x_{1} \in X\right\}
$$

we would have

$$
\inf _{\boldsymbol{\pi}} \mathcal{L}\left(\left(g, \psi_{0}, \psi_{1}\right) ; \boldsymbol{\pi}\right)=-\infty
$$


On the other hand, if $\left(g, \psi_{0}, \psi_{1}\right) \in \Sigma$ the infimum in (11.18) is attained at $\boldsymbol{\pi}=0$ so that

$$
\inf _{\boldsymbol{\pi}} \mathcal{L}\left(\left(g, \psi_{0}, \psi_{1}\right) ; \boldsymbol{\pi}\right)=\int_{X} \psi_{0} \mathrm{~d} \mu_{0}-\int_{X} \psi_{1} \mathrm{~d} \mu_{1}-\frac{1}{p} \int_{X} g^{p} \mathrm{~d} \mathfrak{m}
$$

and therefore (11.18) reads

$$
\mathscr{D}_{q}\left(\mu_{0}, \mu_{1}\right)=\sup \left\{\int_{X} \psi_{0} \mathrm{~d} \mu_{0}-\int_{X} \psi_{1} \mathrm{~d} \mu_{1}-\frac{1}{p} \int_{X} g^{p} \mathrm{~d} \mathfrak{m}:\left(g, \psi_{0}, \psi_{1}\right) \in \Sigma, g>0\right\},
$$

which coincides with (11.12) thanks to (4.29).

Theorem 11.4. Let us suppose that $(X, \tau)$ is compact; then for every $\mu_{0}, \mu_{1} \in \mathcal{M}_{+}(X)$ we have

$$
\mathscr{D}_{q}\left(\mu_{0}, \mu_{1}\right)=\mathrm{pCE}_{p}^{*}\left(\mu_{0}-\mu_{1}\right) .
$$

Proof. Combining (11.12) with (4.30) we easily get

$$
\begin{aligned}
\mathscr{D}_{q}\left(\mu_{0}, \mu_{1}\right)=\sup \{ & \int_{X} \varphi \mathrm{d}\left(\mu_{0}-\mu_{1}\right)-\frac{1}{p} \int_{X} g^{p} \mathrm{dm}: \\
& \left.g \in \mathrm{C}_{b}(X), g>0, \varphi \in \operatorname{Lip}_{b}(X, \tau, \mathrm{d}), \operatorname{lip}_{\mathrm{d}} \varphi \leq g\right\},
\end{aligned}
$$

so that

$$
\mathscr{D}_{q}\left(\mu_{0}, \mu_{1}\right) \stackrel{(11.23)}{\leq} \sup _{\varphi \in \operatorname{Lip}_{b}(X, \tau, \mathrm{d})} \int \varphi \mathrm{d}\left(\mu_{0}-\mu_{1}\right)-\frac{1}{p} \int \operatorname{lip}^{p}(\varphi) \mathrm{dm}=\mathrm{pCE}_{p}^{*}\left(\mu_{0}-\mu_{1}\right) .
$$

Since we already proved that $\mathscr{D}_{q}\left(\mu_{0}, \mu_{1}\right) \geq \mathrm{pCE} E_{p}^{*}\left(\mu_{0}-\mu_{1}\right)$ we conclude.

Corollary 11.5. Let us suppose that $(X, \tau)$ is compact. For every $h \in L^{q}(X, \mathfrak{m})$ with $\int_{X} h \mathrm{~d} \mathfrak{m}=0$ we have

$$
\mathscr{D}_{q}\left(h_{+} \mathfrak{m}, h_{-} \mathfrak{m}\right)=\mathrm{CE}_{p}^{*}(h)={ } \mathrm{CE}_{p}^{*}(h)=\mathrm{pCE}_{p}^{*}(h \mathfrak{m}) .
$$

Proof. Combining (11.9) and (11.7) we know that for every $h \in L^{q}(X, \mathfrak{m})$

$$
\mathscr{D}_{q}\left(h_{+} \mathfrak{m}, h_{-} \mathfrak{m}\right) \geq \mathrm{wCE}_{p}^{*}(h) \geq \mathrm{CE}_{p}^{*}(h) \geq \mathrm{pCE} E_{p}^{*}(h \mathfrak{m}) .
$$

Equality then follows by Theorem 11.4.

By Fenchel-Moreau duality we can now recover for every $f \in L^{p}(X, \mathfrak{m})$

$$
\begin{aligned}
\frac{1}{p} \mathrm{CE}_{p}(f) & =\sup _{h \in L^{q}(X, \mathfrak{m})} \int_{X} h f \mathrm{~d} \mathfrak{m}-\frac{1}{q} \mathrm{CE}_{p}^{*}(h) \\
& =\sup _{h \in L^{q}(X, \mathfrak{m})} \int_{X} h f \mathrm{~d} \mathfrak{m}-\frac{1}{q} \mathrm{wCE}_{p}^{*}(h) \\
& =\frac{1}{p} \mathrm{wCE}_{p}(f)
\end{aligned}
$$

and we obtain the identification of the strong and weak Cheeger energy and of the Sobolev spaces, including the case of a compatible algebra $\mathscr{A}$, thanks to Theorem 6.7. 
Corollary 11.6. Let us suppose that $(X, \tau)$ is compact. Then for every algebra $\mathscr{A}$ compatible with $\mathbb{X}$ we have

$$
H^{1, p}(\mathbb{X}, \mathscr{A})=H^{1, p}(\mathbb{X})=W^{1, p}\left(\mathbb{X}, \mathcal{T}_{q}\right)
$$

with equality of norms; in particular

$$
\mathrm{CE}_{p, \mathscr{A}}(f)=\mathrm{CE}_{p}(f)={ } \mathrm{CE}_{p, \mathcal{T}_{q}}(f) \quad \text { for every } f \in L^{p}(X, \mathfrak{m}),
$$

and for every $f \in W^{1, p}\left(\mathbb{X}, \mathcal{T}_{q}\right)$

$$
|\mathrm{D} f|_{\star, \mathscr{A}}=|\mathrm{D} f|_{\star}=|\mathrm{D} f|_{w, \mathcal{T}_{q}} \quad \mathfrak{m} \text {-a.e. in } X .
$$

\section{The complete case}

Let us now extend the previous result to the case when $(X, \mathrm{~d})$ is complete, by removing the compactness assumption.

Theorem 11.7. Let us suppose that $(X, \mathrm{~d})$ is complete and let $\mathscr{A}$ be an algebra compatible with $\mathbb{X}$. Then the same conclusions (11.25), (11.26) and (11.27) hold.

Proof. Let us consider the Gelfand compactification $\hat{\mathbb{X}}=(\hat{X}, \hat{\tau}, \hat{\mathrm{d}}, \hat{\mathfrak{m}})$ of Theorem 2.34 induced by $\mathscr{A}$. Since $(X, \mathrm{~d})$ is complete, we can apply Theorem 10.32 and we obtain that $\iota_{*}$ induces an isomorphism of $W^{1, p}\left(\mathbb{X}, \mathcal{T}_{q}\right)$ onto $W^{1, p}\left(\hat{\mathbb{X}}, \hat{\mathcal{T}}_{q}\right)$ with

$$
|\mathrm{D} f|_{w, \mathcal{T}_{q}}=\iota^{*}\left(|\mathrm{D} \hat{f}|_{w, \hat{\tau}_{q}}\right), \quad \hat{f}:=\iota_{*} f .
$$

Since $(\hat{X}, \hat{\tau})$ is compact, by Corollary 11.6 we know that $\hat{f} \in H^{1, p}(\hat{X}, \hat{A})$ with

$$
|\mathrm{D} \hat{f}|_{\star, \hat{\mathscr{A}}}=|\mathrm{D} \hat{f}|_{w, \hat{\mathfrak{T}}_{q}} \quad \hat{\mathfrak{m}} \text {-a.e. }
$$

Finally, applying Lemma 5.14 we obtain that $f=\iota^{*} \hat{f}$ belongs to $H^{1, p}(\mathbb{X}, \mathscr{A})$ with

$$
|\mathrm{D} f|_{\star, \mathscr{A}} \leq \iota^{*}\left(|\mathrm{D} \hat{f}|_{\star, \hat{\mathscr{A}}}\right) .
$$

Combining the previous inequalities we obtain

$$
|\mathrm{D} f|_{\star, \mathscr{A}} \leq|\mathrm{D} f|_{w, \mathcal{T}_{q}} \quad \mathfrak{m} \text {-a.e. }
$$

Recalling (10.48) we conclude.

We can also extend to the complete case the dual characterizations of Theorem 11.4 and Corollary 11.5 .

Theorem 11.8. Let us suppose that $(X, \mathrm{~d})$ is complete. Then for every $\mu_{0}, \mu_{1} \in \mathcal{M}_{+}(X)$ we have

$$
\mathscr{D}_{q}\left(\mu_{0}, \mu_{1}\right)=\mathrm{pCE} E_{p}^{*}\left(\mu_{0}-\mu_{1}\right) .
$$

and whenever $\mu_{i}=h_{i} \mathfrak{m}$ with $h_{i} \in L^{q}(X, \mathfrak{m})$ and $h=h_{0}-h_{1}$

$$
\mathscr{D}_{q}\left(h_{0} \mathfrak{m}, h_{1} \mathfrak{m}\right)=\operatorname{CE}_{p}^{*}(h)={ } \mathrm{CE}_{p}^{*}(h)=\mathrm{pCE}_{p}^{*}(h \mathfrak{m}) .
$$


Proof. It is sufficient to prove that $\mathscr{D}_{q}\left(\mu_{0}, \mu_{1}\right) \leq \mathrm{pCE}_{p}^{*}\left(\mu_{0}-\mu_{1}\right)$. Keeping the same notation of the previous proof and using the compactification $\hat{X}$ induced by the canonical algebra $\mathscr{A}=\operatorname{Lip}_{b}(X, \tau, \mathrm{d})$, we consider the Radon measures $\hat{\mu}_{i}:=\iota_{\sharp} \mu_{i} \in \mathcal{M}_{+}(\hat{X})$. It is easy to check that for every plan $\boldsymbol{\pi} \in \Pi\left(\mu_{0}, \mu_{1}\right)$ the push forward $J_{\sharp} \boldsymbol{\pi}$ (where $J(\gamma)=\iota \circ \gamma$ ) belongs to $\Pi\left(\hat{\mu}_{0}, \hat{\mu}_{1}\right)$ in $\operatorname{RA}(\hat{X})$, so that $\mathscr{D}_{q}\left(\mu_{0}, \mu_{1}\right) \leq \mathscr{D}_{q}\left(\hat{\mu}_{0}, \hat{\mu}_{1}\right)$. On the other hand, by Lemma 10.29 and the completeness of $(\iota(X), \hat{\mathrm{d}})$, every plan $\hat{\boldsymbol{\pi}} \in \Pi\left(\hat{\mu}_{0}, \hat{\mu}_{1}\right)$ is concentrated on curves in $J(\mathrm{RA}(X))$ so that $\mathscr{D}_{q}\left(\mu_{0}, \mu_{1}\right)=\mathscr{D}_{q}\left(\hat{\mu}_{0}, \hat{\mu}_{1}\right)$. Recalling that for every $f \in \operatorname{Lip}_{b}(X, \tau, \mathrm{d})$ we have $\hat{f}=\Gamma(f) \in \operatorname{Lip}_{b}(\hat{X}, \hat{\tau}, \hat{\mathrm{d}})$ with $\operatorname{lip}_{\hat{\mathrm{d}}} \hat{f}(\iota(x)) \geq$ $\operatorname{lip}_{\mathrm{d}} f(x)$ we get

$$
\begin{aligned}
\mathscr{D}_{q}\left(\mu_{0}, \mu_{1}\right) & =\mathscr{D}_{q}\left(\hat{\mu}_{0}, \hat{\mu}_{1}\right)=\sup _{\hat{f} \in \operatorname{Lip}_{b}(\hat{X}, \hat{\tau}, \hat{\mathrm{d}})} \int_{\hat{X}} \hat{f} \mathrm{~d}\left(\hat{\mu}_{0}-\hat{\mu}_{1}\right)-\frac{1}{p} \int_{\hat{X}}\left(\operatorname{Lip}_{\hat{\mathrm{d}}} \hat{f}(\hat{x})\right)^{p} \mathrm{~d} \hat{\mathfrak{m}}(\hat{x}) \\
& =\sup _{\hat{f} \in \operatorname{Lip}_{b}(\hat{X}, \hat{\tau}, \hat{\mathrm{d}})} \int_{X} \hat{f} \circ \iota \mathrm{d}\left(\mu_{0}-\mu_{1}\right)-\frac{1}{p} \int_{X}\left(\operatorname{Lip}_{\hat{\mathrm{d}}} \hat{f}(\iota(x))^{p} \mathrm{~d} \mathfrak{m}(x)\right. \\
& \leq \sup _{f \in \operatorname{Lip}_{b}(X, \tau, \mathrm{d})} \int_{X} f \mathrm{~d}\left(\mu_{0}-\mu_{1}\right)-\frac{1}{p} \int_{X}\left(\operatorname{Lip}_{\mathrm{d}} f(x)\right)^{p} \mathrm{dm}(x)=\operatorname{pCE}_{p}^{*}\left(\mu_{0}-\mu_{1}\right) .
\end{aligned}
$$

As a consequence of the above result, we can prove a simple characterization of nontriviality for the Cheeger energy.

Theorem 11.9. Let us suppose that $(X, \mathrm{~d})$ is complete. The following properties are equivalent:

(a) The Cheeger energy is trivial: $\mathrm{CE}_{p}(f) \equiv 0$ for every $f \in L^{p}(X, \mathfrak{m})$.

(b) The Cheeger energy $\mathrm{CE}_{p}(f)$ is finite for every $f \in L^{p}(X, \mathfrak{m})$.

(c) $\mathrm{RA}_{0}(X)$ is $\mathcal{T}_{q}$-negligible.

(d) $\mathrm{RA}_{0}(X)$ is $\mathcal{B}_{q}$-negligible (equivalently, if $(X, \tau)$ is Souslin, $\mathrm{RA}_{0}(X)$ is $\operatorname{Mod}_{p}$-negligible).

Proof. The implication $(a) \Rightarrow(b)$ is obvious.

$(\boldsymbol{b}) \Rightarrow(\boldsymbol{a})$ If the Cheeger energy is always finite then the Sobolev norm of $H^{1, p}(\mathbb{X}) \subset L^{p}(X, \mathfrak{m})$ is equivalent to the $L^{p}$-norm [21, Corollary 2.8], so that there exists a constant $C>0$ such that

$$
\mathrm{CE}_{p}(f) \leq C\|f\|_{L^{p}(X, \mathfrak{m})}^{p} \quad \text { for every } f \in L^{p}(X, \mathfrak{m}) .
$$

Let us show that (11.34) implies $\mathrm{CE}_{p}(f) \equiv 0$ for every $f \in H^{1, p}(\mathbb{X})$. We consider the 2 periodic Lipschitz function $\phi: \mathbb{R} \rightarrow \mathbb{R}$ satisfying $\phi(r)=|r|$ for $r \in[-1,1]$ and we set

$$
\phi_{n}(r):=\phi(n r), \quad f_{n}(x):=\phi_{n}(f(x)) .
$$

Thanks to the locality of the minimal relaxed gradient we have $\left|\mathrm{D} f_{n}\right|_{\star}(x)=n|\mathrm{D} f|_{\star}(x)$ so that

$$
\mathrm{CE}_{p}(f)=\frac{1}{n^{p}} \mathrm{CE}_{p}\left(f_{n}\right) \leq \frac{C}{n^{p}}\left\|f_{n}\right\|_{L^{p}(X, \mathfrak{m})}^{p} \leq \frac{C}{n^{p}} \mathfrak{m}(X) \rightarrow 0 \quad \text { as } n \rightarrow \infty .
$$

(c) $\Rightarrow$ (a) If (c) holds then for every nonvanishing $h \in L^{p}(X, \mathfrak{m})$ the class $\Pi\left(h_{+} \mathfrak{m}, h_{-} \mathfrak{m}\right)$ is empty so that $\mathrm{CE}_{p}^{*}(h)=\mathscr{D}_{q}\left(h_{+} \mathfrak{m}, h_{-} \mathfrak{m}\right)=+\infty$. By duality we obtain $\mathrm{CE}_{p} \equiv 0$.

$(a) \Rightarrow(c)$ Let $\boldsymbol{\pi} \in \mathcal{T}_{q}$ with $\boldsymbol{\pi}(\mathrm{RA}(X))>0$ and let $\left(\mathrm{d}_{i}\right)_{i \in I}$ be a directed family of continuous semidistances as in $(2.24 \mathrm{a}, \mathrm{b}, \mathrm{c}, \mathrm{d})$. It is not restrictive to assume that $\boldsymbol{\pi}$ is concentrated on a compact 
set $\Gamma$ on which $\ell$ is continuous. The image $K=\mathrm{e}(\Gamma)$ is compact in $(X, \tau)$ : for every $i \in I$ we can find a countable set $K_{i} \subset K$ such that for every $x \in K \inf _{y \in K_{i}} \mathrm{~d}_{i}(x, y)=0$. For every $y \in K_{i}$ the minimal relaxed gradient of the function $x \mapsto \mathrm{d}_{i}(x, y)$ vanishes, so that there exists a $\pi$-negligible set $N_{i} \subset \Gamma$ such that the function $t \mapsto \mathrm{d}_{i}\left(R_{\gamma}(t), y\right)$ is constant for every $y \in K_{i}$ and $\gamma \in \Gamma \backslash N_{i}$. By continuity we deduce that $t \mapsto \mathrm{d}_{i}\left(R_{\gamma}(t), y\right)$ is constant for every $y \in K$ so that $\mathrm{d}_{i}\left(R_{\gamma}(t), \gamma_{0}\right)=0$ for every $\gamma \in \Gamma \backslash N_{i}$; by integration we obtain

$$
\int\left(\int_{\gamma} \mathrm{d}_{i}\left(x, \gamma_{0}\right)\right) \mathrm{d} \boldsymbol{\pi}(\gamma)=0
$$

On the other hand, for every $\gamma \in \Gamma$ Beppo Levi's Monotone Convergence Theorem yields $\lim _{i \in I} \int_{\gamma} \mathrm{d}_{i}\left(x, \gamma_{0}\right)=$ $\int_{\gamma} \mathrm{d}\left(x, \gamma_{0}\right)$. A further application of the same theorem thanks to the fact that the function $\gamma \mapsto$ $\int_{\gamma} \mathrm{d}_{i}\left(x, \gamma_{0}\right)$ is continuous on $\Gamma$ with respect to the $\tau_{\mathrm{A}}$ topology yields

$$
0=\lim _{i \in I} \int\left(\int_{\gamma} \mathrm{d}_{i}\left(x, \gamma_{0}\right)\right) \mathrm{d} \boldsymbol{\pi}(\gamma)=\int\left(\int_{\gamma} \mathrm{d}\left(x, \gamma_{0}\right)\right) \mathrm{d} \boldsymbol{\pi}(\gamma)
$$

which shows that $\pi$-a.e. $\gamma$ is constant, a contradiction.

$(\boldsymbol{d}) \Leftrightarrow(\boldsymbol{c})$ The implication $(d) \Rightarrow(c)$ is obvious. In order to prove the converse one, we argue by contradiction and we suppose that there exists a plan $\pi \in \mathcal{B}_{q}$ with $\pi\left(\operatorname{RA}_{0}(X)\right)>0$. We can then argue as in the proof of Proposition 10.17 and define a new plan $\tilde{\boldsymbol{\pi}} \in \mathcal{T}_{q}$ according to (10.33). It is clear that $\tilde{\boldsymbol{\pi}}\left(\mathrm{RA}_{0}(X)\right)>0$ as well.

\subsection{Notes}

The representation theorems 11.3, 11.4, and 11.8 are new. The proof of Theorem $H=W$ has been given in [58] in the case of doubling, $p$-Poincaré spaces [17, Theorem 5.1] and in $[9,8]$ for general spaces by a completely different method: it relies on three basic ingredients:

- the properties of the $L^{2}$-gradient flow of the Cheeger energy (in particular the comparison principle),

- the estimate of the Wasserstein velocity of the evolution curve, by means of a suitable version of the Kuwada's Lemma,

- the representation of the solution as the evaluation at time $t$ of a dynamic plan concentrated on curves with finite $q$-energy,

- the derivation of the Shannon-Reny entropy along the flow, by using the weak upper gradients of the solutions.

It is curious that the refined estimates of the Hopf-Lax flow play a crucial role in the second step.

A different proof of Theorem 11.9 in the context of Newtonian spaces can be found in [46, Prop. 7.1.33].

\section{Examples and applications}

\subsection{Refined invariance of the (strong) Cheeger energy}

Invariance w.r.t. the algebra $\mathscr{A}$

Theorem 12.1 (Invariance of the Cheeger energy w.r.t. $\mathscr{A}$ ). For every e.m.t.m. space $\mathbb{X}$ and every compatible algebra $\mathscr{A}$ the Sobolev space $H^{1, p}(\mathbb{X}, \mathscr{A})$ is independent of the compatible algebra $\mathscr{A}$ and coincides with $H^{1, p}(\mathbb{X})$.

Proof. It is sufficient to combine Theorem 11.7 with Corollary 5.16. 
We can rephrase the previous statement as a density result: if $\mathscr{A}$ is a compatible algebra for $\mathbb{X}$,

for every $f \in H^{1, p}(\mathbb{X})$ there exists a sequence $f_{n} \in \mathscr{A}$ such that

$$
f_{n} \rightarrow f, \quad \operatorname{lip} f_{n} \rightarrow|\mathrm{D} f|_{\star} \quad \text { strongly in } L^{p}(X, \mathfrak{m}) .
$$

We can also slightly relax the assumption that $\mathscr{A}$ is unital.

Proposition 12.2. Let $\mathbb{X}=(X, \tau, \mathrm{d}, \mathfrak{m})$ be an e.m.t.m. space and let $\mathscr{A} \subset \operatorname{Lip}_{b}(X, \tau, \mathrm{d})$ be an algebra of functions satisfying (2.52) (we do not assume that $\mathbb{1} \in \mathscr{A}$ ). If there exists a sequence of compact sets $K_{n} \subset X$ and functions $f_{n} \in \mathscr{A}$ such that

$$
f_{n}(x) \geq 1 \quad \text { for every } x \in K_{n}, \quad \lim _{n \rightarrow \infty} \int_{X \backslash K_{n}}\left(1+\left|\operatorname{lip} f_{n}(x)\right|^{p}\right) \operatorname{dm}(x)=0
$$

then $\mathscr{A}$ satisfies (12.1).

Since $\mathfrak{m}$ is tight, (12.2) is surely satisfied if for every compact $K \subset X$ there exists a function $f \in \mathscr{A}$ such that

$$
f(x) \geq 1 \quad \text { for every } x \in K, \quad \operatorname{Lip}(f, X) \leq C \quad \text { for a constant } C \text { independent of } K .
$$

Proof of Proposition 12.2. Let $K_{n}, f_{n}$ be satisfying (12.2) and let $\left[-c_{n}, c_{n}\right] \supset f_{n}(X)$. Choosing a sequence $i \mapsto \varepsilon_{i} \downarrow 0$ we can consider the polynomial $P_{n, i}=2 P_{\varepsilon_{i}}^{c_{n},-1 / 2,1 / 2}$ where $P_{\varepsilon}^{c, \alpha, \beta}$ is given by Corollary 2.24. Notice that $P_{n, i}(0)=0$ so that the functions $h_{n, i}:=P_{n, i} \circ f_{n}$ belong to $\mathscr{A}$. It is easy to check that

$$
\lim _{i \rightarrow \infty} h_{n, i}=f_{n}^{\prime}:=\left(-1 \vee 2 f_{n} \wedge 1\right), \quad \operatorname{lip} h_{n, i} \leq 2 \operatorname{lip} f_{n},
$$

$\lim _{i \rightarrow \infty} \operatorname{lip} h_{n, i}(x)=\lim _{i \rightarrow \infty} P_{n, i}^{\prime}\left(f_{n}(x)\right) \operatorname{lip} f_{n}(x)=0 \quad$ for every $x$ in a neighborhood of $K_{n}$.

By Lebesgue Dominated Convergence Theorem we get

$$
\begin{gathered}
\lim _{i \rightarrow \infty} \int_{K_{n}}\left|h_{n, i}-1\right|^{p} \mathrm{~d} \mathfrak{m}=0, \quad \lim _{i \rightarrow \infty} \int_{K_{n}}\left|\operatorname{lip} h_{n, i}\right|^{p} \mathrm{~d} \mathfrak{m}=0, \\
\int_{X \backslash K_{n}}\left(\left|h_{n, i}-1\right|^{p}+\left|\operatorname{lip} h_{n, i}\right|^{p}\right) \mathrm{d} \mathfrak{m} \leq 2^{p} \int_{X \backslash K_{n}}\left(1+\left|\operatorname{lip} f_{n}\right|^{p}\right) \mathrm{d} \mathfrak{m} .
\end{gathered}
$$

We can now introduce the algebra $\tilde{\mathscr{A}}=\mathscr{A} \oplus\{a \mathbb{1}\}=\{\tilde{f}=f+c \mathbb{1}: f \in \mathscr{A}, c \in \mathbb{R}\}$ which is clearly unital and compatible with $\mathbb{X}$ according to definition 2.17. Applying (12.1) to $\tilde{\mathscr{A}}$, for every $f \in H^{1, p}(\mathbb{X})$ we can find a sequence $\tilde{f}_{k}=f_{k}+a_{k} \mathbb{1} \in \tilde{\mathscr{A}}, k \in \mathbb{N}$, such that

$$
\tilde{f}_{k} \rightarrow f, \quad \operatorname{lip} \tilde{f}_{k} \rightarrow|\mathrm{D} f|_{\star} \quad \text { strongly in } L^{p}(X, \mathfrak{m}) \quad \text { as } k \rightarrow \infty .
$$

For every $k>0$, by (12.6) and (12.2) we can find $i=i(k)$ and $n=n(k)$ sufficiently big, such that $u_{k}:=h_{n, i} \in \mathscr{A}$ such that

$$
-1 \leq u_{k} \leq 1, \quad a_{k}^{p} \int_{X}\left(\left|u_{k}-1\right|^{p}+\left|\operatorname{lip} u_{k}\right|^{p}\right) \mathrm{d} \mathfrak{m} \leq 1 / k^{p}
$$

We can then consider $f_{k}^{\prime}:=f_{k}+a_{k} u_{k} \in \mathscr{A}$, observing that

$$
\begin{aligned}
\left\|f_{k}^{\prime}-\tilde{f}_{k}\right\|_{p} & \leq a_{k}\left\|u_{k}-\mathbb{1}\right\|_{L^{p}(X, \mathfrak{m})} \leq 1 / k, \\
\left\|\operatorname{lip} f_{k}^{\prime}\right\|_{L^{p}(X, \mathfrak{m})} & \leq\left\|\operatorname{lip} \tilde{f}_{k}\right\|_{L^{p}(X, \mathfrak{m})}+a_{k}\left\|\operatorname{lip} u_{k}\right\|_{L^{p}(X, \mathfrak{m})} \leq\left\|\operatorname{lip} \tilde{f}_{k}\right\|_{L^{p}(X, \mathfrak{m})}+1 / k .
\end{aligned}
$$


We conclude that the sequence $\left(f_{k}^{\prime}\right)_{k \in \mathbb{N}} \subset \mathscr{A}$ satisfies

$$
\lim _{k \rightarrow \infty}\left\|f_{k}^{\prime}-f\right\|_{L^{p}(X, \mathfrak{m})}=0, \quad \lim _{k \rightarrow \infty} \int_{X}\left|\operatorname{lip} f_{k}^{\prime}\right|^{p} \mathrm{~d} \mathfrak{m}=\mathrm{CE}_{p}(f) .
$$

Let us show two simple examples of applications of Proposition 12.2 and condition (12.3):

1. If $\mathrm{d}$ is $\tau$-continuous, one can always consider the algebra

$$
\operatorname{Lip}_{b s}(X, \tau, \mathrm{d}):=\{f \in \operatorname{Lip}(X, \tau, \mathrm{d}): f \text { has d-bounded support }\}
$$

2. If $(X, \mathrm{~d})$ is locally compact (and thus $\tau$ is the topology induced by $\mathrm{d}$ ) then the algebra

$$
\operatorname{Lip}_{c}(X, \tau, \mathrm{d}):=\{f \in \operatorname{Lip}(X, \tau, \mathrm{d}): f \text { has compact support }\}
$$

satisfies (12.1).

\section{Invariance w.r.t. measure-preserving embeddings}

Let us now consider the invariance of the strong Cheeger energy w.r.t. measure preserving embeddings. Thanks to the previous Theorem 12.1 it is sufficient to consider the case of the canonical algebra.

Theorem 12.3 (Invariance of the (strong) Cheeger energy w.r.t. measure preserving embeddings). Let $\mathbb{X}=(X, \tau, \mathrm{d}, \mathfrak{m})$ and $\mathbb{X}^{\prime}=\left(X^{\prime}, \tau^{\prime}, \mathrm{d}^{\prime}, \mathfrak{m}^{\prime}\right)$ be two e.m.t.m. spaces and let $\iota: X \rightarrow X^{\prime}$ be a measure preserving embedding of $\mathbb{X}$ into $\mathbb{X}^{\prime}$ according to Definition 2.28. Then $\iota^{*}$ is an isomorphism between $H^{1, p}\left(\mathbb{X}^{\prime}\right)$ onto $H^{1, p}(\mathbb{X})$ and

$$
\text { for every } f=\iota^{*} f^{\prime} \in H^{1, p}(\mathbb{X}) \quad|\mathrm{D} f|_{\star}=\iota^{*}\left(|\mathrm{D} f|_{\star}^{\prime}\right) .
$$

Proof. Let $\overline{\mathbb{X}}$ and $\overline{\mathbb{X}}^{\prime}$ be the completion of $\mathbb{X}$ and $\mathbb{X}^{\prime}$ (where $X$ and $X^{\prime}$ can be identified as $\mathrm{d}$ and $\mathrm{d}^{\prime}$ dense subsets of $\bar{X}$ and $\bar{X}^{\prime}$ respectively, see Remark 2.37). Since $\iota: X \rightarrow X^{\prime}$ is an isometry and $X$ is d-dense in $\bar{X}, \iota$ can be extended to an isometric embedding $\bar{\iota}$ of $\bar{X}$ into $\bar{X}^{\prime}$. Using property (C4) of Corollary 2.36 one can check that $\bar{\iota}$ is also continuous from $(\bar{X}, \bar{\tau})$ to $\left(\bar{X}^{\prime}, \bar{\tau}^{\prime}\right)$ and since $\bar{X} \backslash X$ and $\bar{X}^{\prime} \backslash X^{\prime}$ are $\overline{\mathfrak{m}}$ and $\overline{\mathfrak{m}}^{\prime}$ negligible subsets respectively, we also see that $\bar{\iota}$ is measure-preserving. We conclude that $\iota$ is a measure-preserving imbedding of $\bar{X}$ into $\bar{X}^{\prime}$.

Since the Cheeger energy is invariant w.r.t. completion by Corollary 5.16, the above argument shows that it is not restrictive to assume that $\mathbb{X}$ and $\mathbb{X}^{\prime}$ are complete. By Theorem 11.7 out thesis follows by the property for the spaces $W^{1, p}\left(\mathbb{X}, \mathcal{T}_{q}\right)$ and $W^{1, p}\left(\mathbb{X}^{\prime}, \mathcal{T}_{q}^{\prime}\right)$ and the corresponding weak upper gradients, proved in Theorem 10.32.

Recalling the examples of 2.30, we obtain two useful properties:

Corollary 12.4 (Invariance w.r.t. the topology). Let $\mathbb{X}=(X, \tau, \mathrm{d}, \mathfrak{m})$ be an e.m.t.m. space and let $\tau^{\prime}$ be a coarser topology such that $\left(X, \tau^{\prime}, \mathrm{d}\right)$ is an e.m.t. space. Then $H^{1, p}(X, \tau, \mathrm{d}, \mathfrak{m})$ is isomorphic to $H^{1, p}\left(X, \tau^{\prime}, \mathrm{d}, \mathfrak{m}\right)$ with equal minimal relaxed gradients.

Corollary 12.5 (Restriction). Let $\mathbb{X}=(X, \tau, \mathrm{d}, \mathfrak{m})$ be an e.m.t.m. space and let $Y \subset X$ be a mmeasurable subset of $X$ with $\mathfrak{m}(X \backslash Y)=0$. If $\mathbb{Y}$ is the associated e.m.t.m. space according to Example 2.30(d), $H^{1, p}(\mathbb{X})$ is isomorphic to $H^{1, p}(\mathbb{Y})$ with equal minimal relaxed gradients. In particular, $H^{1, p}(X, \tau, \mathrm{d}, \mathfrak{m})$ is always isomorphic to $H^{1, p}(\operatorname{supp}(\mathfrak{m}), \tau, \mathrm{d}, \mathfrak{m})$. 


\section{Invariance w.r.t. the length and the conformal constructions}

Thanks to Theorem 11.7, we can extend the results of Lemma 10.33 and Proposition 10.35 to the Cheeger energy.

Corollary 12.6. Let $\mathbb{X}=(X, \tau, \mathrm{d}, \mathfrak{m})$ be a complete e.m.t.m. space and let $\delta: X \times X \rightarrow[0,+\infty]$ be an extended distance such that

$$
(X, \tau, \delta) \text { is an extended metric-topological space, } \quad \mathrm{d} \leq \delta \leq \mathrm{d}_{\ell} \quad \text { in } X \times X .
$$

Then $H^{1, p}(X, \tau, \mathrm{d}, \mathfrak{m})=H^{1, p}(X, \tau, \delta, \mathfrak{m})$ and the corresponding minimal relaxed gradients coincide.

Corollary 12.7. Let $\mathbb{X}=(X, \tau, \mathrm{d}, \mathfrak{m})$ be a complete e.m.t.m. space, let $g \in \mathrm{C}_{b}(X)$ with $0<m_{g} \leq$ $g \leq M_{g}<\infty$, and let $\delta: X \times X \rightarrow[0,+\infty]$ be an extended distance such that

$$
\mathbb{X}^{\prime}=(X, \tau, \delta, \mathfrak{m}) \text { is an e.m.t.m. space, } \quad \mathrm{d}_{g}^{\prime} \leq \delta \leq \mathrm{d}_{g} \quad \text { in } X \times X .
$$

A function $f \in L^{p}(X, \mathfrak{m})$ belongs to the Sobolev space $H^{1, p}\left(\mathbb{X}^{\prime}\right)$ if and only if $f \in H^{1, p}(\mathbb{X})$, and the corresponding minimal relaxed gradients in $\mathbb{X}$ and in $\mathbb{X}^{\prime}$ (which we call $|\mathrm{D} f|_{\star, \mathbb{X}}$ and $|\mathrm{D} f|_{\star, \mathbb{X}^{\prime}}$ respectively) satisfy

$$
|\mathrm{D} f|_{\star, \mathbb{X}}=g^{-1}|\mathrm{D} f|_{\star, \mathbb{X}^{\prime}}
$$

\subsection{Examples}

Example 12.8 (Sobolev spaces in $\mathbb{R}^{d}$ or in a Finsler-Riemannian manifold). Let us consider the space $X:=\mathbb{R}^{d}$ with the usual topology $\tau$, the distance $\mathrm{d}$ induced by a norm $\|\cdot\|$ with dual norm $\|\cdot\|_{*}$, and a finite positive Borel measure $\mathfrak{m}$.

Being $\left(\mathbb{R}^{d},\|\cdot\|\right)$ complete the weak and strong Sobolev spaces coincide. By Proposition 12.2 we can choose

$$
\mathscr{A}:=\mathrm{C}_{c}^{\infty}\left(\mathbb{R}^{d}\right), \quad \operatorname{lip} f(x)=\|\mathrm{D} f(x)\|_{*} \quad \text { for every } f \in \mathscr{A} .
$$

We thus obtain

$$
\begin{aligned}
& H^{1, p}\left(\mathbb{R}^{d}, \tau,\|\cdot\|, \mathfrak{m}\right)=\left\{f \in L^{p}\left(\mathbb{R}^{d}, \mathfrak{m}\right): \exists f_{n} \in \mathrm{C}_{c}^{\infty}\left(\mathbb{R}^{d}\right)\right. \\
&\left.f_{n} \rightarrow f \text { in } L^{p}\left(X, \mathbb{R}^{d}\right), \sup _{n} \int_{\mathbb{R}^{d}}\left\|\mathrm{D} f_{n}\right\|_{*}^{p} \mathrm{~d} \mathfrak{m}<\infty\right\} .
\end{aligned}
$$

It is not difficult to check that this space is always reflexive (see also [1] and Corollary 12.11) and it is an Hilbert space if $\|\cdot\|$ is induced by a scalar product and $p=2$, since $\mathrm{pCE}_{2}$ is a quadratic form on $\mathscr{A}$. In this case we obtain the Sobolev space introduced by [20]. At least when the gradient operator is closable in $L^{p}(X, \mathfrak{m})$, the present metric approach also coincides with the definition of weighted Sobolev spaces given in [44] (a proof of the equivalence under doubling and Poincaré assumptions has been given in [17, Appendix 2])

A completely analogous approach can be used in a complete Finsler or Riemannian manifold.

Example 12.9 (Sobolev space on a separable Banach space). Let $(B,\|\cdot\|)$ be a separable Banach space endowed with the strong topology $\tau_{s}$ and the distance $\mathrm{d}$ induced by the norm. Let $\mathfrak{m}$ be a finite positive Borel measure in $B$ and $\mathbb{B}:=\left(B, \tau_{s}, \mathrm{~d}, \mathfrak{m}\right)$. We can consider the algebra $\mathscr{A}=\mathrm{Cyl}(B)$ of smooth cylindrical functions (see Example 2.19) so that

$$
\operatorname{lip} f(x)=\|\mathrm{D} f(x)\|_{*} \quad \text { for every } f \in \mathscr{A}
$$


and therefore

$$
\begin{aligned}
& H^{1, p}(\mathbb{B})=\left\{f \in L^{p}(H, \mathfrak{m}): \exists f_{n} \in \mathscr{A}\right. \\
&\left.f_{n} \rightarrow f \text { in } L^{p}(B, \mathfrak{m}), \sup _{n} \int_{B}\left\|\mathrm{D} f_{n}\right\|_{*}^{p} \mathrm{~d} \mathfrak{m}<\infty\right\} .
\end{aligned}
$$

We can give an equivalent intrinsic characterization in terms of vector valued Sobolev differentials, in the case $B$ is also reflexive. Some of the results below could be extended to the case when $B$ has the Radon-Nikodym property [27]. If $\boldsymbol{h}: B \rightarrow B^{\prime}$ is a Borel map (recall the definition given in $\S 2.1$ and $\S$ A.5 in the Appendix) it is not difficult to check that for every $\gamma \in \operatorname{RA}(B)$ we have $t \mapsto\left\langle\boldsymbol{h}\left(R_{\gamma}(t)\right), R_{\gamma}^{\prime}(t)\right)$ is Lebesgue-measurable. If $\int_{\gamma}\|\boldsymbol{h}\|_{*}<\infty$ we can thus consider the curvilinear integral

$$
\int_{\gamma}\langle\boldsymbol{h}, \dot{\gamma}\rangle:=\int_{0}^{1}\left\langle\boldsymbol{h}\left(R_{\gamma}(t)\right), R_{\gamma}^{\prime}(t)\right\rangle \mathrm{d} t
$$

We will denote by $L^{p}\left(B, \mathfrak{m} ; B^{\prime}\right)$ the Bochner space of Borel $\mathfrak{m}$-measurable maps $\boldsymbol{h}: B \rightarrow B^{\prime}$ such that

$$
\int_{B}\|\boldsymbol{h}(x)\|_{*}^{p} \mathrm{~d} \mathfrak{m}(x)<\infty
$$

which is the dual of the Bochner space $L^{q}(B, \mathfrak{m} ; B)$ [28, Theorem 8.20.3].

Given a function $f \in L^{p}(B, \mathfrak{m})$ we say that a Borel map $\boldsymbol{g} \in L^{p}\left(B, \mathfrak{m} ; B^{\prime}\right)$ is a $\mathcal{T}_{q}$-weak gradient of $f$ if

$$
f\left(\gamma_{1}\right)-f\left(\gamma_{0}\right)=\int_{\gamma}\langle\boldsymbol{g}, \dot{\gamma}\rangle \quad \text { for } \mathcal{T}_{q} \text {-a.e. } \gamma \in \operatorname{RA}(B)
$$

Notice that the integral in (12.20) is well defined since the fact that $\|\boldsymbol{g}\|_{*} \in L^{q}(B ; \mathfrak{m})$ yields $\int_{\gamma}\|\boldsymbol{g}\|_{*}<$ $\infty$ for $\mathcal{T}_{q}$-a.e. $\gamma \in \mathrm{RA}(B)$. Arguing as in 10.6 , we can show that the class of weak gradients is invariant w.r.t. modifications in a $\mathfrak{m}$-negligible subset. We will use the symbols

$$
\begin{aligned}
\mathrm{WG}_{p}(f) & :=\left\{\boldsymbol{g} \in L^{p}\left(B, \mathfrak{m} ; B^{\prime}\right): \boldsymbol{g} \text { is a weak gradient of } f\right\} \\
\mathrm{WG}_{p} & :=\left\{(f, \boldsymbol{g}) \in L^{p}(B, \mathfrak{m}) \times L^{p}\left(B, \mathfrak{m} ; B^{\prime}\right): \boldsymbol{g} \in \mathrm{WG}_{p}(f)\right\} .
\end{aligned}
$$

Every curve $\gamma \in \operatorname{RA}(B)$ induces a vector measure $\boldsymbol{\nu}_{\gamma} \in \mathcal{M}(B ; B)$ defined by

$$
\int_{B} f \mathrm{~d} \boldsymbol{\nu}_{\gamma}:=\int_{0}^{1} f\left(R_{\gamma}(t)\right) R_{\gamma}^{\prime}(t) \mathrm{d} t \quad \text { for every } f \in \mathrm{B}_{b}(B),
$$

whose total variation is bounded by $\nu_{\gamma}:\left|\boldsymbol{\nu}_{\gamma}\right| \leq \nu_{\gamma}$. If $\boldsymbol{\pi} \in \mathcal{M}_{+}(\mathrm{RA}(B))$ is a dynamic plan we can then consider the vector measure

$$
\boldsymbol{\mu}_{\boldsymbol{\pi}}:=\int_{B} \boldsymbol{\nu}_{\gamma} \mathrm{d} \boldsymbol{\pi}(\gamma), \quad\left|\boldsymbol{\mu}_{\boldsymbol{\pi}}\right| \leq \mu_{\boldsymbol{\pi}}
$$

If $\boldsymbol{\pi} \in \mathcal{B}_{q}$ then there exists a function $\boldsymbol{h}_{\boldsymbol{\pi}} \in L^{q}(B, \mathfrak{m} ; B)$ such that

$$
\boldsymbol{\mu}_{\boldsymbol{\pi}}=\boldsymbol{h}_{\boldsymbol{\pi}} \mathfrak{m}, \quad \int_{B} f \mathrm{~d} \boldsymbol{\mu}_{\boldsymbol{\pi}}=\int_{B} f(x) \boldsymbol{h}_{\boldsymbol{\pi}}(x) \mathrm{d} \mathfrak{m} .
$$

Theorem 12.10. Let us suppose that $B$ is a separable and reflexive Banach space and let $f \in$ $L^{p}(B, \mathfrak{m})$. 
(a) If $f \in \mathrm{C}^{1}(B) \cap \operatorname{Lip}(B)$ then $\mathrm{D} f \in \mathrm{WG}_{p}(f)$.

(b) A function $\boldsymbol{g} \in L^{p}\left(B, \mathfrak{m} ; B^{\prime}\right)$ belongs to $\mathrm{WG}_{p}(f)$ if and only if for every $\boldsymbol{\pi} \in \mathcal{B}_{q}$ with $\boldsymbol{\mu}_{\boldsymbol{\pi}}=$ $h_{\pi} \mathfrak{m}$

$$
\int_{B} f \mathrm{~d}\left(\pi_{1}-\pi_{0}\right)=\int_{B}\left\langle\boldsymbol{g}(x), \boldsymbol{h}_{\boldsymbol{\pi}}(x)\right\rangle \mathrm{d} \mathfrak{m} .
$$

(c) The set $\mathrm{WG}_{p}$ is a (weakly) closed linear space of $L^{p}(B, \mathfrak{m}) \times L^{p}\left(B, \mathfrak{m} ; B^{\prime}\right)$.

(d) If $(f, \boldsymbol{g}) \in \mathrm{WG}_{p}$ then $g:=\|\boldsymbol{g}\|_{*}$ is a $\mathcal{T}_{q}$-weak upper gradient of $f$. Conversely, if $g$ is a $(p, \mathrm{Cyl}(B))$-relaxed gradient of $f$ then there exists $\boldsymbol{g} \in \mathrm{WG}_{p}(f)$ such that $\|\boldsymbol{g}\|_{*} \leq g$.

(e) A function $f$ belongs to the Sobolev space $H^{1, p}(\mathbb{B})$ if and only if there exists a weak gradient $\boldsymbol{g} \in L^{p}\left(B, \mathfrak{m} ; B^{\prime}\right)$. In this case $\mathrm{WG}_{p}(f)$ has a unique element of minimal norm $\mathrm{D}_{\mathfrak{m}} f$,

$$
|\mathrm{D} f|_{\star}=\left\|\mathrm{D}_{\mathfrak{m}} f\right\|_{*} \quad \mathfrak{m} \text {-a.e., } \quad \mathrm{CE}_{p}(f)=\int_{B}\left\|\mathrm{D}_{\mathfrak{m}} f\right\|_{*}^{p} \mathrm{~d} \mathfrak{m},
$$

and there exists a sequence $f_{n} \in \operatorname{Cyl}(B)$ such that

$$
\lim _{n \rightarrow \infty}\left\|f_{n}-f\right\|_{L^{p}(B, \mathfrak{m})}+\left\|\mathrm{D} f_{n}-\mathrm{D}_{\mathfrak{m}} f\right\|_{L^{p}\left(B, \mathfrak{m} ; B^{\prime}\right)}=0 .
$$

Proof. (a) is an obvious consequence of the chain rule of $f$ along a Lipschitz curve.

(b) follows by the same argument of the proof of Lemma 10.8.

(c) is an immediate consequence of (12.23).

(d) The first statement is a consequence of (12.23), which yields

$$
\int_{B} f \mathrm{~d}\left(\pi_{1}-\pi_{0}\right) \leq \int_{B}\|\boldsymbol{g}\|_{*}\left\|\boldsymbol{h}_{\boldsymbol{\pi}}\right\| \mathrm{d} \mathfrak{m}=\int_{B}\|\boldsymbol{g}\|_{*} \mathrm{~d}\left(\left|\boldsymbol{\mu}_{\boldsymbol{\pi}}\right|\right) \leq \int_{B}\|\boldsymbol{g}\|_{*} \mathrm{~d} \mu_{\boldsymbol{\pi}}
$$

so that $\|\boldsymbol{g}\|_{*}$ is a $\mathcal{T}_{q}$-weak upper gradient by Lemma 10.8 .

Conversely, let $g$ be a $(p, \operatorname{Cyl}(B))$-relaxed gradient of $f$. By definition, there exists a sequence $f_{n}$ of cylindrical functions such that $f_{n} \rightarrow f$ in $L^{p}(B, \mathfrak{m})$ and $\operatorname{lip} f_{n} \rightarrow \tilde{g}$ in $L^{p}(B, \mathfrak{m})$ with $\tilde{g} \leq g$. Since $f_{n}$ are cylindrical, lip $f_{n}(x)=\left\|\mathrm{D} f_{n}(x)\right\|_{*} ;$ since $L^{p}\left(B, \mathfrak{m} ; B^{\prime}\right)$ is reflexive, there exists a subsequence (still denoted by $f_{n}$ ) such that $\mathrm{D} f_{n} \rightarrow \boldsymbol{g}$ in $L^{p}\left(B, \mathfrak{m} ; B^{\prime}\right)$. Thanks to claim $(\mathrm{c}),(f, \boldsymbol{g})$ belongs to $\mathrm{WG}_{p}$ and the weak lower semicontinuity of continuous convex functionals in a reflexive space yields for every Borel set $A \subset B$

$$
\int_{A}\|\boldsymbol{g}\|_{*} \mathrm{~d} \mathfrak{m} \leq \liminf _{n \rightarrow \infty} \int_{A}\left\|\mathrm{D} f_{n}\right\|_{*} \mathrm{~d} \mathfrak{m}=\int_{A} \tilde{g} \mathrm{~d} \mathfrak{m} \leq \int_{A} g \mathrm{~d} \mathfrak{m}
$$

so that $\|\boldsymbol{g}\|_{*} \leq g \mathfrak{m}$-a.e.

(e) The first statement follows by Claim (d) and the identification Theorem 11.7 between $H^{1, p}(\mathbb{B})$ and $W^{1, p}\left(\mathbb{B}, \mathcal{T}_{q}\right)$. Claim $(\mathrm{d})$ and the strict convexity of the $L^{p}\left(B, \mathfrak{m} ; B^{\prime}\right)$ norm yields (12.24). The proof of Claim (d) also shows that there exists a sequence $f_{n} \in \operatorname{Cyl}(B)$ such that $\left(f_{n}, \mathrm{D} f_{n}\right)$ weakly converges to $\left(f, \mathrm{D}_{\mathfrak{m}} f\right)$ in $L^{p}(B, \mathfrak{m}) \times L^{p}\left(B, \mathfrak{m} ; B^{\prime}\right)$. We can now apply Mazur Theorem.

Corollary 12.11. If $B$ is a reflexive Banach space then $H^{1, p}(\mathbb{B})$ is reflexive. If moreover $B$ is an Hilbert space then $H^{1,2}(\mathbb{B})$ is an Hilbert space. 
Proof. The proof that $H^{1, p}(\mathbb{B})$ is reflexive is standard: we first notice that $\mathrm{WG}_{p}$ is a weakly closed subset of the reflexive space $L^{p}(B, \mathfrak{m}) \times L^{p}\left(B, \mathfrak{m} ; B^{\prime}\right)$ and the projection on the first component $\mathrm{p}:(f, \boldsymbol{g}) \rightarrow f$ is a continuous and surjective map from $\mathrm{WG}_{p}$ onto $H^{1, p}(\mathbb{B})$ satisfying $\|f\|_{H^{1, p}(\mathbb{B})}=$ $\min \left\{\|(f, \boldsymbol{g})\|_{\mathrm{WG}_{p}}: \mathrm{p}(f, \boldsymbol{g})=f\right\}$. If $L$ is a bounded linear functional on $H^{1, p}(\mathbb{B})$ then $L \circ \mathrm{p}$ belongs to $\mathrm{WG}_{p}^{\prime}$. If $f_{n}$ is a bounded sequence in $H^{1, p}(\mathbb{B})$ then there exists a subsequence $k \mapsto f_{n(k)}$ and limits $(f, \boldsymbol{g}) \in \mathrm{WG}_{p}$ such that $\left(f_{n(k)}, \mathrm{D}_{\mathfrak{m}} f_{n(k)}\right) \rightarrow(f, \boldsymbol{g})$ in $L^{p}(B, \mathfrak{m}) \times L^{p}\left(B, \mathfrak{m} ; B^{\prime}\right)$. It follows that

$$
\left.\lim _{k \rightarrow \infty} L\left(f_{n(k)}\right)=\lim _{k \rightarrow \infty} L \circ \mathrm{p}\left(f_{n(k)}, \mathrm{D}_{\mathfrak{m}} f_{n(k)}\right)\right)=\lim _{k \rightarrow \infty} L \circ \mathrm{p}(f, \boldsymbol{g})=L(f) .
$$

Remark 12.12. The same conclusion of the previous Corollary holds even if $X$ is a closed subset of a reflexive and separable Banach (or Hilbert) space $\mathbb{B}$ endowed with the induced length distance $\mathrm{d}_{\ell}$ (and, e.g., the strong topology $\left.\tau_{s}\right)$. In this case we have $W^{1, p}\left(X, \tau_{s}, \mathrm{~d}_{\ell}, \mathfrak{m}\right)=W^{1, p}\left(X, \tau_{s}, \mathrm{~d}, \mathfrak{m}\right)$ by Lemma 10.33 (see also 10.34 ), $W^{1, p}\left(X, \tau_{s}, \mathrm{~d}, \mathfrak{m}\right)=H^{1, p}\left(X, \tau_{s}, \mathrm{~d}, \mathfrak{m}\right)$ by Theorem 11.7 , and eventually $H^{1, p}\left(X, \tau_{s}, \mathrm{~d}, \mathfrak{m}\right)=H^{1, p}\left(\mathbb{B}, \tau_{s}, \mathrm{~d}, \mathfrak{m}\right)$ by Corollary 12.5 . We can then apply Corollary 12.11 .

Remark 12.13. If we consider the closed subspace

$$
\mathrm{WG}_{p, o}:=\{0\} \times \mathrm{WG}_{p}(0)=\left\{(0, \boldsymbol{g}): \boldsymbol{g} \in L^{p}\left(B, \mathfrak{m} ; B^{\prime}\right): \int_{B}\left\langle\boldsymbol{g}, \boldsymbol{h}_{\boldsymbol{\pi}}\right\rangle \mathrm{d} \mathfrak{m}=0 \quad \text { for every } \boldsymbol{\pi} \in \mathcal{T}_{q}\right\}
$$

it would not be difficult to see that $H^{1, p}(\mathbb{B})$ is isomorphic to the quotient space $\mathrm{WG}_{p} / \mathrm{WG}_{p, o}$. The operator $f \mapsto \mathrm{D} f$ from $\operatorname{Cyl}(B)$ to $L^{p}\left(B, \mathfrak{m} ; B^{\prime}\right)$ is closable if and only if $\mathrm{WG}_{p, o}=0$. As typical example one can consider the case of an Hilbert space $H$ endowed with a log-concave probability measure $\mathfrak{m}$ (in particular a Gaussian measure), see e.g. [14], [24].

Example 12.14 (Wiener space). Let $(X,\|\cdot\|)$ be a separable Banach space endowed with its strong topology $\tau$ and let $\mathfrak{m}$ be a centered non-degenerate Radon Gaussian measure. For every bounded linear functional $v \in X^{\prime}$ let us set

$$
R_{\mathfrak{m}}(v):=\int_{X}|\langle v, x\rangle|^{2} \mathrm{~d} \mathfrak{m}(x)
$$

$R_{\mathfrak{m}}$ is a nondegenerate continuous quadratic form on $X^{\prime}$, whose dual characterizes the CameronMartin space $H(\mathfrak{m})$ as the subset of $X$ where the functional

$$
|x|_{H(\mathfrak{m})}=\sup \left\{\langle v, x\rangle: v \in X^{\prime}, R_{\mathfrak{m}}(v) \leq 1\right\},
$$

is finite, and thus defines a Hilbertian norm. We also set $\mathrm{d}(x, y):=|x-y|_{H(\mathfrak{m})}$. As we have seen in Example 2.12, $\mathbb{X}=(X, \tau, \mathrm{d}, \mathfrak{m})$ is an Polish e.m.t.m. space. By using the algebra $\mathscr{A}=\mathrm{Cyl}(X)$ of smooth cylindrical functions it is not difficult to see that for every $f \in \mathscr{A}$ we have $\operatorname{D} f(x) \in X^{\prime}$ and

$$
\operatorname{lip}_{\mathfrak{d}} f(x)=\sup _{v \in H(\mathfrak{m}),|v| \leq 1}\langle\mathrm{D} f(x), v\rangle=\left(R_{\mathfrak{m}}(\mathrm{D} f(x))^{1 / 2}=|\mathrm{D} f(x)|_{H(\mathfrak{m})^{\prime}}\right.
$$

so that the metric Sobolev space $H^{1, p}(\mathbb{X})$ coincides with the usual Sobolev space $W^{1, p}(\mathfrak{m})$ [18] defined as the completion of the cylindrical functions with respect to the norm

$$
\|f\|_{W^{1, p}(\mathfrak{m})}^{p}:=\int_{X}\left(|f(x)|^{p}+|\mathrm{D} f(x)|_{H(\mathfrak{m})^{\prime}}^{p}\right) \mathrm{d} \mathfrak{m}(x)
$$




\subsection{Distinguished representations of metric Sobolev spaces}

We have already seen that the strong Cheeger energy is invariant w.r.t. completion of the underlying space. We can now use Theorem 12.3 to obtain isomorphic realizations of the Sobolev space $H^{1, p}(\mathbb{X})$ with special e.m.t.m. space $\mathbb{X}$. Let us first fix the property we are interested in.

Definition 12.15 (Isomorphic representations of Sobolev spaces). Let $\mathbb{X}, \mathbb{X}^{\prime}$ be two e.m.t.m. spaces. We say that $H^{1, p}\left(\mathbb{X}^{\prime}\right)$ is an isomorphic representation of $H^{1, p}(\mathbb{X})$ if there exists a linear isomorphism $\iota^{*}: H^{1, p}\left(\mathbb{X}^{\prime}\right) \rightarrow H^{1, p}(\mathbb{X})$ satisfying (12.10) induced by a measure preserving embedding $\iota: X \rightarrow X^{\prime}$ from $\mathbb{X}$ into $\mathbb{X}^{\prime}$.

All the statements below refers to an arbitrary e.m.t.m. space $\mathbb{X}=(X, \tau, \mathrm{d}, \mathfrak{m})$ and to the strong Sobolev space $H^{1, p}(\mathbb{X})$. Starting from a complete space, they also provide equivalent representations for the weak Sobolev space $W^{1, p}\left(\mathbb{X}, \mathcal{T}_{q}\right)$ thanks to Theorem 11.7.

A first example has already been used in the proof of Theorem 11.7. It is sufficient to use the compactification Theorem 2.34.

Corollary 12.16 (Compact representation). Every Sobolev space $H^{1, p}(\mathbb{X})$ admits an isomorphic representation $H^{1, p}(\hat{\mathbb{X}})$ where $\hat{X}$ is a compact e.m.t.m. space.

Corollary 12.17. Suppose that $(X, \tau)$ is a Souslin space. Then there exists a separable Banach space $\left(B,\|\cdot\|_{B}\right)$ and a weakly* compact convex subset $\Sigma$ of the dual unit ball of $B^{\prime}$ such that $H^{1, p}(\mathbb{X})$ admits an isomorphic representation as $H^{1, p}\left(\Sigma, \tau_{w *}, \mathrm{~d}_{B^{\prime}}, \mathfrak{m}_{B}\right)$ where $\tau_{w *}$ is the weak topology of $B^{\prime}\left(\left(\Sigma, \tau_{w *}\right)\right.$ is a compact geodesic metric space $)$ and $\mathrm{d}_{B^{\prime}}(v, w):=\|v-w\|_{B^{\prime}}$. Moreover, we can choose the compatible algebra $\mathscr{A}$ of the smooth cylindrical functions generated by the elements of $B$ (as linear functional on $B^{\prime}$ ).

Proof. Since $(X, \tau)$ is Souslin, we can find a metrizable and separable auxiliary topology $\tau^{\prime}$ and a compatible algebra $\mathscr{A} \subset \operatorname{Lip}\left(X, \tau^{\prime}, \mathrm{d}\right)$ which is countably generated. We can then apply the Gelfand compactification Theorem 2.34 with the construction described by Proposition 2.33. Since $B$ is the closure of $\mathscr{A}$ in $\mathrm{C}_{b}\left(X, \tau^{\prime}\right), B$ is a separable Banach space and $\Sigma$ is a compact convex subset of the unit ball of $B^{\prime}$.

\section{A Appendix}

\section{A.1 Nets}

We recap here a few basic facts about nets (see e.g. [54, p.187-188]). Let $I$ be a directed set, i.e. a set endowed with a partial order $\preceq$ satisfying

$$
\begin{gathered}
i \preceq i ; \quad i \preceq j, j \preceq k \quad \Rightarrow \quad i \preceq k \quad \text { for every } i, j, k \in I, \\
\forall i, j \in I \quad \exists k \in I: \quad i \preceq k, j \preceq k .
\end{gathered}
$$

As subset $J \subset I$ is called cofinal if for every $i \in I$ there exists $j \in J$ such that $i \preceq j$.

If $\left(Y, \tau_{Y}\right)$ is a Hausdorff topological space, a net in $Y$ is a map $y: I \rightarrow Y$ defined in some directed set $I$; the notation $\left(y_{i}\right)_{i \in I}$ (or simply $\left(y_{i}\right)$ ) is often used to denote a net.

The net $\left(y_{i}\right)_{i \in I}$ converges to an element $y \in Y$ and we write $y_{i} \rightarrow y$ or $\lim _{i \in I} y_{i}=y$ if for every neighborhood $U$ of $y$ there exists $i_{0} \in I$ such that $i_{0} \preceq i \Rightarrow y_{i} \in U$.

$y$ is an accumulation point of $\left(y_{i}\right)$ if for every neighborhood $U$ of $y$ the set of indexes $\{i \in I$ : $\left.y_{i} \in U\right\}$ is cofinal. 
A subnet $\left(y_{\mathfrak{i}(j)}\right)_{j \in J}$ of $\left(y_{i}\right)$ is obtained by a composition $y \circ \mathfrak{i}$ where $\mathfrak{i}: J \rightarrow I$ is a map defined in a directed set $J$ satisfying

$$
j_{1} \preceq j_{2} \Rightarrow \mathfrak{i}\left(j_{1}\right) \preceq \mathfrak{i}\left(j_{2}\right), \quad \mathfrak{i}(J) \text { is cofinal in } I .
$$

Nets are a useful substitution of the notion of sequences, when the topology $\tau_{Y}$ does not satisfy the first countable axiom. In particular we have the following properties:

(a) A point $y$ belongs to the closure of a subset $A \subset Y$ if and only if there exists a net of points of $A$ converging to $y$.

(b) A function $f: Y \rightarrow Z$ between Hausdorff topological spaces is continuous if and only if for every net $\left(y_{i}\right)_{i \in I}$ converging to $y$ in $Y$ we have $\lim _{i \in I} f\left(y_{i}\right)=f(y)$.

(c) $y$ is an accumulation point of $\left(y_{i}\right)$ if and only if there exists a subnet $\left(y_{\mathfrak{i}(j)}\right)_{j \in J}$ such that $\lim _{j \in J} y_{\mathfrak{i}(j)}=y$.

(d) $\left(Y, \tau_{Y}\right)$ is compact if and only if every net in $Y$ has a convergent subnet.

\section{A.2 Initial topologies}

Let $\left(Y, \tau_{Y}\right)$ be a Hausdorff topological space and let $\mathcal{F} \subset \mathrm{C}(Y)$ be a collection of real continuous functions separating the points of $Y$. We say that $\tau_{Y}$ is generated by $\mathcal{F}$ if it is the coarsest topology for which all the functions of $\mathcal{F}$ are continuous (thus $\tau_{Y}$ coincides with the initial or weak topology induced by $\mathcal{F}$ ). A basis for the topology $\tau_{Y}$ is generated by the finite intersections of sets of the form $\left\{f^{-1}(U): f \in \mathcal{F}, U\right.$ open in $\left.\mathbb{R}\right\}$.

An important property of topologies generated by a separating family of functions is the characterization of convergence: for every net $\left(y_{i}\right)_{i \in I}$ in $Y$

$$
\lim _{i \in I} y_{i}=y \text { in } Y \quad \Leftrightarrow \quad \lim _{i \in I} f\left(y_{i}\right)=f(y) \quad \text { for every } f \in \mathcal{F} \text {. }
$$

It is also easy to check that such topologies are completely regular: if $F$ is a closed set and $y \in Y \backslash F$, we can find $f_{1}, \cdots, f_{N} \in \mathcal{F}$ and open sets $U_{1}, \cdots U_{N} \in \mathbb{R}$ such that $y \in \cap_{n=1}^{N} f_{n}^{-1}\left(U_{n}\right) \subset Y \backslash F$. Up to compositions with affine maps, it is not restrictive to assume that $f_{n}(y)=1$ and $U_{n} \supset(0,2)$ so that the function $f(x):=0 \vee \min _{1 \leq n \leq N} f_{n}(x)\left(2-f_{n}(x)\right)$ satisfies $f(y)=1$ and $\left.f\right|_{Y \backslash F} \equiv 0$.

\section{A.3 Polish, Lusin, Souslin and Analytic sets.}

Denote by $\mathbb{N}^{\infty}$ the collection of all infinite sequences of natural numbers and by $\mathbb{N}_{0}^{\infty}$ the collection of all finite sequences $\left(n_{0}, \ldots, n_{i}\right)$, with $i \geq 0$ and $n_{i}$ natural numbers. Let $\mathcal{A} \subset \mathfrak{P}(Y)$ containing the empty set (typical examples are, in a topological space $\left(Y, \tau_{Y}\right)$, the classes $\mathscr{F}(Y), \mathscr{K}(Y), \mathscr{B}(Y)$ of closed, compact, and Borel sets respectively). We call table of sets (or Souslin scheme) in $\mathcal{A}[19$, Definition 1.10.1] a map A associating to each finite sequence $\left(n_{0}, \ldots, n_{i}\right) \in \mathbb{N}_{0}^{\infty}$ a set $\mathrm{A}_{\left(n_{0}, \ldots, n_{i}\right)} \in$ $\mathcal{A}$.

Definition A.1 ( $\mathcal{A}$-analytic sets). $S \subset Y$ is said to be $\mathcal{A}$-analytic if there exists a table A of sets in $\mathcal{A}$ such that

$$
S=\bigcup_{(n) \in \mathbb{N} \infty} \bigcap_{i=0}^{\infty} \mathrm{A}_{\left(n_{0}, \ldots, n_{i}\right)} .
$$

The collection of all the $\mathcal{A}$-analytic sets will be denoted by $\mathrm{S}(\mathcal{A})$. 
Let us recall a list of useful properties (see $[19, \S 1.10]$ )

(A1) Countable unions and countable intersections of elements of $\mathcal{A}$ belongs to $\mathrm{S}(\mathcal{A})$.

(A2) $\mathrm{S}(\mathrm{S}(\mathcal{A}))=\mathrm{S}(\mathcal{A})$

(A3) If the complement of every set of $\mathcal{A}$ belongs to $\mathrm{S}(\mathcal{A})$ then $\mathrm{S}(\mathcal{A})$ contains the $\sigma$-algebra generated by $\mathcal{A}$. In particular, in a metrizable space $Y \mathscr{B}(Y)$-analytic sets are $\mathscr{F}(Y)$-analytic.

(A4) In a topological space $(E, \tau), \mathscr{B}(E)$-analytic sets are universally measurable [19, Theorem 1.10.5], i.e. they are $\mu$-measurable for any finite Borel measure $\mu$.

Definition A.2 ([57, Chap. II]). An Hausdorff topological space $\left(Y, \tau_{Y}\right)$ (in particular, a subset of a topological space $(X, \tau)$ with the relative topology) is a Polish space if it is separable and $\tau_{Y}$ is induced by a complete metric $\mathrm{d}_{Y}$ on $Y$.

$\left(Y, \tau_{Y}\right)$ is said to be Souslin (resp. Lusin) if it is the image of a Polish space under a continuous (resp. injective and continuous) map.

Differently from the Borel property, notice that the Souslin and Lusin properties for subsets of a topological space are intrinsic, i.e. they depend only on the induced topology.

We recall a few important properties of the class of Souslin and Lusin sets.

Proposition A.3. The following properties hold:

(a) In a Hausdorff topological space $\left(Y, \tau_{Y}\right)$, Souslin sets are $\mathscr{F}(Y)$-analytic; if $\mathscr{S}(Y)$ denotes the class of Souslin sets, $\mathrm{S}(\mathscr{S}(Y))=\mathscr{S}(Y)$.

(b) if $\left(Y, \tau_{Y}\right)$ is a Souslin space (in particular if it is a Polish or a Lusin space), the notions of Souslin and $\mathscr{F}(Y)$-analytic sets coincide and in this case Lusin sets are Borel and Borel sets are Souslin;

(c) if $Y, Z$ are Souslin spaces and $f: Y \rightarrow Z$ is a Borel injective map, then $f^{-1}$ is Borel;

(d) if $Y, Z$ are Souslin spaces and $f: Y \rightarrow Z$ is a Borel map, then $f$ maps Souslin sets to Souslin sets.

(e) If $\left(Y, \tau_{Y}\right)$ is Souslin then every finite nonnegative Borel measure in $Y$ is Radon.

Proof. (a) is proved in [19, Theorems 6.6.6, 6.6.8]. In connection with (b), the equivalence between Souslin and $\mathscr{F}(E)$-analytic sets is proved in [19, Theorem 6.7.2], the fact that Borel sets are Souslin in [19, Corollary 6.6.7] and the fact that Lusin sets are Borel in [19, Theorem 6.8.6]. (c) and (d) are proved in [19, Theorem 6.7.3]. For (e) we refer to [57, Thm. $9 \& 10$, p. 122].

Since in Souslin spaces $\left(Y, \tau_{Y}\right)$ we have at the same time tightness of finite Borel measures and coincidence of Souslin and $\mathscr{F}(E)$-analytic sets, the measurability of $\mathscr{B}(E)$-analytic sets yields in particular that for every $\mu \in \mathcal{M}_{+}(Y)$

$$
\mu(B)=\sup \{\mu(K): K \in \mathscr{K}(Y), K \subset B\} \quad \text { for every } B \in \mathscr{S}(Y) .
$$

We will also recall another useful property [57, Pages 103-105].

Lemma A.4. Let us suppose that $\left(Y, \tau_{Y}\right)$ is a Souslin space. 
(a) $Y$ is strongly Lindeöf, i.e. every open cover of an open set has a countable sub-cover.

(b) Every family $\mathcal{F}$ of lower semicontinuous real functions defined in $Y$ has a countable subfamily $\left(f_{n}\right)_{n \in \mathbb{N}} \subset \mathcal{F}$ such that $\sup _{f \in \mathcal{F}} f(x)=\sup _{n \in \mathbb{N}} f_{n}(x)$ for every $x \in Y$.

(c) If $Y$ is regular, every open set is an $F_{\sigma}$ (countable intersection of closed set), thus in particular is $\mathscr{F}(Y)$-analytic.

(d) If $Y$ is completely regular, there exists a metrizable and separable topology $\tau^{\prime}$ coarser than $\tau_{Y}$.

\section{A.4 Choquet capacities}

Let us recall the definition of a Choquet capacity in related to a collection $\mathcal{A}$ of subsets of $Y$ containing the empty set and closed under finite unions and countable intersections. [25, Chap. III, $\S 2$ ].

Definition A.5. A function $\mathcal{J}: \mathfrak{P}(Y) \rightarrow[0,+\infty]$ is a Choquet $\mathcal{A}$-capacity if it satisfies the properties

(C1) J is increasing: $A \subset B \quad \Rightarrow \quad \mathcal{J}(A) \leq \mathcal{J}(B)$.

(C2) For every increasing sequence $A_{n} \subset Y: \mathcal{J}\left(\cup_{n} A_{n}\right)=\lim _{n \rightarrow \infty} \mathcal{J}\left(A_{n}\right)$.

(C3) For every decreasing sequence $K_{n} \in \mathcal{A}: \mathcal{J}\left(\cap_{n} K_{n}\right)=\lim _{n \rightarrow \infty} \mathcal{J}\left(K_{n}\right)$.

A subset $A \subset Y$ is called capacitable if $\mathcal{J}(A)=\sup \{\mathcal{J}(K): K \subset A, K \in \mathcal{A}\}$.

Theorem A.6 (Choquet, [25, Chap. III, 28]). If $\mathcal{J}$ is a $\mathcal{A}$-capacity then every $\mathcal{A}$-analytic set is capacitable.

\section{A.5 Measurable maps with values in separable Banach spaces}

Let $\left(Y, \tau_{Y}\right)$ be a Hausdorff topological space endowed with a Radon measure $\mu \in \mathcal{M}_{+}(Y)$ and let $\left(V,\|\cdot\|_{V}\right)$ be a separable Banach space with dual $V^{\prime}$. Since $V$ is a Polish space, the classes of strong and weak Borel sets coincide.

A map $h: Y \rightarrow V$ is Borel $\mu$-measurable (recall the definition given in $\S 2.1$ ) then it is also Lusin $\mu$-measurable, since $V$ is metrizable; in particular, $\boldsymbol{h}$ admits a Borel representative $\tilde{\boldsymbol{h}}$ such that $\mathfrak{m}(\tilde{\boldsymbol{h}} \neq \boldsymbol{h})=0$. If $\int_{Y}\|\boldsymbol{h}\| \mathrm{d} \mathfrak{m}<\infty$ then $\boldsymbol{h}$ is also Bochner integrable, i.e. there exists a sequence $\boldsymbol{h}_{n}: Y \rightarrow V$ of simple Borel functions such that

$$
\lim _{n \rightarrow \infty} \int_{Y}\left\|\boldsymbol{h}_{n}-\boldsymbol{h}\right\| \mathrm{d} \mathfrak{m}=0
$$

We can then define its Bochner integral $\int_{Y} \boldsymbol{h} \mathrm{d} \mu$ as the $\operatorname{limit}_{n \rightarrow \infty} \int_{Y} \boldsymbol{h}_{n} \mathrm{dm}$ and the corresponding vector measure $\mu_{h}:=h \mu$ defined by

$$
\boldsymbol{\mu}_{\boldsymbol{h}}(A):=\int_{A} \boldsymbol{h} \mathrm{d} \boldsymbol{\mu} \text { for every } \mu \text {-measurable set } A \subset Y .
$$




\section{A.6 Homogeneous convex functionals}

Let us first recall a simple property of $p$-homogeneous convex functionals.

Lemma A.7 (Dual of $p$-homogeneous functionals). Let $C$ be a convex cone of some vector space $V$, $p>1$, and $\phi, \psi: C \rightarrow[0, \infty]$ with $\psi=\phi^{1 / p}, \phi=\psi^{p}$. We have the following properties:

(a) $\phi$ is convex and p-homogeneous (i.e. $\phi(\kappa v)=\kappa^{p} \phi(v)$ for every $\kappa \in \mathbb{R}$ and $v \in C$ ) in $C$ if and only if $\psi$ is convex and 1-homogeneous on $C$ (a seminorm, if $C$ is a vector space and $\psi$ is finite).

(b) Under one of the above equivalent assumptions, setting for every linear functional $z: V \rightarrow \mathbb{R}$

$$
\frac{1}{q} \phi^{*}(z):=\sup _{v \in C}\langle z, v\rangle-\frac{1}{p} \phi(v), \quad \psi_{*}(z):=\sup \{\langle z, v\rangle: v \in C, \psi(v) \leq 1\},
$$

we have

$$
\psi_{*}(z)=\inf \{c \geq 0:\langle z, v\rangle \leq c \psi(v) \quad \text { for every } v \in C\}, \quad \phi^{*}(z)=\left(\psi_{*}(z)\right)^{q},
$$

where in the first infimum we adopt the convention inf $A=+\infty$ if $A$ is empty.

Proof. By setting $\phi(v)=\psi(v)=+\infty$ if $v \in V \backslash C$, it is not restrictive to assume that $C=V$. 1. Let us assume that $\phi$ is convex and $p$-homogeneous: we want to prove that $\psi$ is a seminorm (this is the only nontrivial implication). Since $\psi$ is 1-homogeneous, it is sufficient to prove that it is convex. Let $v_{i} \in V, i=0,1$, with $r_{i}:=\psi\left(v_{i}\right)+\varepsilon$ for $\varepsilon>0$, so that $\tilde{v}_{i}:=v_{i} / r_{i}$ satisfies $\psi\left(\tilde{v}_{i}\right)<1$. We fix $\alpha_{i} \geq 0$ with $\sum_{i} \alpha_{i}=1$ and we set $r:=\sum_{i} \alpha_{i} r_{i}$ and $\beta_{i}:=\alpha_{i} r_{i} / r$ which still satisfy $\beta_{i} \geq 0$ and $\sum_{i} \beta_{i}=1$. Since the set $K:=\{\psi(v) \leq 1\}=\{\phi(v) \leq 1\}$ is convex we have $\sum \beta_{i} \tilde{v}_{i} \in K$. It follows that $\psi\left(\sum_{i} \beta_{i} \tilde{v}_{i}\right) \leq 1$; on the other hand $\sum_{i} \beta_{i} \tilde{v}_{i}=\frac{1}{r} \sum_{i} \alpha_{i} v_{i}$ and therefore $\psi\left(\sum_{i} \alpha_{i} v_{i}\right)=r \psi\left(\sum_{i} \beta_{i} \tilde{v}_{i}\right) \leq r=\varepsilon+\sum_{i} \alpha_{i} \psi\left(v_{i}\right)$. Since $\varepsilon>0$ is arbitrary, we conclude.

2. We set $K_{a}:=\{v \in V: \psi(v)=a\}, a \in\{0,1\}$, and observe that

$$
\psi_{*}(z)=\delta_{K_{0}}(z)+\sup _{v \in K_{1}}\langle z, v\rangle
$$

where

$$
\delta_{K_{0}}(z)=\sup _{v \in K_{0}}\langle z, v\rangle= \begin{cases}0 & \text { if }\langle z, v\rangle \equiv 0 \forall v \in K_{0} \\ +\infty & \text { otherwise }\end{cases}
$$

Similarly

$$
\frac{1}{q} \phi^{*}(z)=\delta_{K_{0}}(z)+\sup _{v \in V \backslash K_{0}}\left(\langle z, v\rangle-\frac{1}{p} \phi(v)\right) .
$$

Since $V \backslash K_{0}=\bigcup_{\kappa \in \mathbb{R}} \kappa K_{1}$ we have

$$
\begin{aligned}
\frac{1}{q} \phi^{*}(z) & =\delta_{K_{0}}(z)+\sup _{v \in K_{1}, \kappa \in \mathbb{R}} \kappa\langle z, v\rangle-\frac{\kappa^{p}}{p} \phi(v)=\delta_{K_{0}}(z)+\sup _{v \in K_{1}} \sup _{\kappa \in \mathbb{R}}\left(\kappa\langle z, v\rangle-\frac{\kappa^{p}}{p}\right) \\
& =\delta_{K_{0}}(z)+\frac{1}{p} \sup _{v \in K_{1}}(\langle z, v\rangle)^{p}=\left(\psi_{*}(z)\right)^{p} .
\end{aligned}
$$




\section{A.7 Von Neumann theorem}

Let $\mathbb{A}, \mathbb{B}$ be convex sets of some vector spaces and let $\mathcal{L}: \mathbb{A} \times \mathbb{B} \rightarrow \mathbb{R}$ be a saddle function satisfying

$$
\begin{aligned}
a \mapsto \mathcal{L}(a, b) & \text { is concave in } \mathbb{A} \text { for every } b \in \mathbb{B}, \\
b \mapsto \mathcal{L}(a, b) & \text { is convex in } \mathbb{B} \text { for every } a \in \mathbb{A} .
\end{aligned}
$$

It is always true that

$$
\inf _{b \in \mathbb{B}} \sup _{a \in \mathbb{A}} \mathcal{L}(a, b) \geq \sup _{a \in \mathbb{A}} \inf _{b \in \mathbb{B}} \mathcal{L}(a, b) .
$$

The next result provides an important sufficient condition to guarantee the equality in (A.8): we use a formulation which is slightly more general than the statement of [59, Thm. 3.1], but it follows by the same argument.

Theorem A.8 (Von Neumann). Let us suppose that (A.6), (A.7) hold, that $\mathbb{B}$ is endowed with some Hausdorff topology and that there exists $a_{\star} \in \mathbb{A}$ and $C_{\star}>\sup _{a \in \mathbb{A}} \inf _{b \in \mathbb{B}} \mathcal{L}(a, b)$ such that

$$
\begin{aligned}
& \mathbb{B}_{\star}:=\left\{b \in \mathbb{B}: \mathcal{L}\left(a_{\star}, b\right) \leq C_{\star}\right\} \quad \text { is not empty and compact in } \mathbb{B}, \\
& b \mapsto \mathcal{L}(a, b) \text { is lower semicontinuous in } \mathbb{B}_{\star} \text { for every } a \in \mathbb{A} .
\end{aligned}
$$

Then

$$
\min _{b \in \mathbb{B}} \sup _{a \in \mathbb{A}} \mathcal{L}(a, b)=\sup _{a \in \mathbb{A}} \inf _{b \in \mathbb{B}} \mathcal{L}(a, b) .
$$

Similarly, if $\mathbb{A}$ is endowed with a Hausdorff topology and there exists $b_{\star} \in \mathbb{B}$ and $D_{\star}<\inf _{b \in \mathbb{B}} \sup _{a \in \mathbb{A}} \mathcal{L}(a, b)$ such that

$$
\begin{aligned}
\mathbb{A}_{\star} & :=\left\{a \in \mathbb{A}: \mathcal{L}\left(a, b_{\star}\right) \geq D_{\star}\right\} \quad \text { is not empty and compact in } \mathbb{A}, \\
& a \mapsto \mathcal{L}(a, b) \text { is upper semicontinuous in } \mathbb{A}_{\star} \text { for every } b \in \mathbb{B} .
\end{aligned}
$$

Then

$$
\inf _{b \in \mathbb{B}} \sup _{a \in \mathbb{A}} \mathcal{L}(a, b)=\max _{a \in \mathbb{A}} \inf _{b \in \mathbb{B}} \mathcal{L}(a, b) .
$$

We reproduce here the main part of the proof of (A.11); (A.14) follows simply by considering the Lagrangian $\tilde{\mathcal{L}}(b, a):=-\mathcal{L}(a, b)$ in $\mathbb{B} \times \mathbb{A}$ and inverting the role of $\mathbb{A}$ and $\mathbb{B}$.

Proof. Let $s:=\sup _{a \in \mathbb{A}} \inf _{b \in \mathbb{B}} \mathcal{L}(a, b)$ and let $\mathbb{B}_{a}:=\{b \in \mathbb{B}: \mathcal{L}(a, b) \leq s\}, \mathbb{B}_{a \star}:=\left\{b \in \mathbb{B}: \mathcal{L}\left(a_{\star}, b\right) \leq s\right\}$. We notice that $\mathbb{B}_{a \star} \subset \mathbb{B}_{\star}$ and that for every $a \in \mathbb{A}$ the set $\mathbb{B}_{a} \cap \mathbb{B}_{\star}=\left\{b \in \mathbb{B}_{\star}: \mathcal{L}(a, b) \leq s\right\}$ is compact thanks to (A.9) and (A.10). If $A \subset \mathbb{A}$ is a collection containing $a_{\star}$ then

$$
\mathbb{B}_{A}=\bigcap_{a \in A} \mathbb{B}_{a}=\bigcap_{a \in A}\left(\mathbb{B}_{a} \cap \mathbb{B}_{a \star}\right)=\bigcap_{a \in A}\left(\mathbb{B}_{a} \cap \mathbb{B}_{\star}\right)
$$

so that $\mathbb{B}_{A}$ is a (possibly empty) compact set. The thesis follows if we check that $\mathbb{B}_{\mathbb{A}}$ contains a point $\bar{b}$, since in that case $\inf _{b \in \mathbb{B}} \sup _{a \in \mathbb{A}} \mathcal{L}(a, b) \leq \sup _{a \in \mathbb{A}} \mathcal{L}(a, \bar{b}) \leq s$ by construction; on the other hand, (A.8) shows that $\sup _{a \in \mathbb{A}} \mathcal{L}(a, \bar{b})=s \leq \sup _{a \in \mathbb{A}} \mathcal{L}(a, b)$ for every $b \in \mathbb{B}$, so that the minimum in the left-hand side of (A.11) is attained at $\bar{b}$. 
Since $\mathbb{B}_{A}$ are compact whenever $a_{\star} \in A$, it is sufficient to prove that for every finite collection $A=\left\{a_{1}, \cdots, a_{n}\right\}$ containing $a_{\star}$ the intersection $\mathbb{B}_{A}$ is not empty. To this aim, since $b \mapsto \mathcal{L}\left(a_{k}, b\right)$ are convex functions, [59, Lemma 2.1] yields

$$
\inf _{b \in \mathbb{B}} \sup _{1 \leq k \leq n} \mathcal{L}\left(a_{k}, b\right)=\inf _{b \in \mathbb{B}} \sum_{k=1}^{N} \chi_{k} \mathcal{L}\left(a_{k}, b\right)
$$

for a suitable choice of nonnegative coefficients $\chi_{k} \in[0,1]$ with $\sum_{k=1}^{n} \chi_{k}=1$. We thus get by concavity

$$
\inf _{b \in \mathbb{B}} \sum_{k=1}^{N} \chi_{k} \mathcal{L}\left(a_{k}, b\right) \leq \inf _{b \in \mathbb{B}} \mathcal{L}\left(\sum_{k=1}^{N} \chi_{k} a_{k}, b\right) \leq s,
$$

so that $\inf _{b \in \mathbb{B}} \sup _{1 \leq k \leq n} \mathcal{L}\left(a_{k}, b\right) \leq s$. On the other hand, since $\sup _{1 \leq k \leq n} \mathcal{L}\left(a_{k}, b\right) \geq \mathcal{L}\left(a_{\star}, b\right)$, every $b \in \mathbb{B}$ such that $\sup _{1 \leq k \leq n} \mathcal{L}\left(a_{k}, b\right) \leq C_{\star}$ belongs to $\mathbb{B}_{\star}$ so that $C_{\star}>s$ yields

$$
s=\inf _{b \in \mathbb{B}} \sup _{1 \leq k \leq n} \mathcal{L}\left(a_{k}, b\right)=\inf _{b \in \mathbb{B}_{\star}} \sup _{1 \leq k \leq n} \mathcal{L}\left(a_{k}, b\right)=\min _{b \in \mathbb{B}_{\star}} \sup _{1 \leq k \leq n} \mathcal{L}\left(a_{k}, b\right),
$$

where in the last identity we used the fact that $\mathbb{B}_{\star}$ is compact and that the restriction of the function $b \mapsto \sup _{1 \leq k \leq n} \mathcal{L}\left(a_{k}, b\right)$ to $\mathbb{B}_{\star}$ is lower semicontinuous. We conclude that $\bigcap_{k=1}^{N}\left\{b \in \mathbb{B}: \mathcal{L}\left(a_{k}, b\right) \leq\right.$ $s\}$ is not empty.

\section{References}

[1] L. Ambrosio, M. Colombo, and S. Di Marino. Sobolev spaces in metric measure spaces: reflexivity and lower semicontinuity of slope. In Variational methods for evolving objects, volume 67 of Adv. Stud. Pure Math., pages 1-58. Math. Soc. Japan, [Tokyo], 2015.

[2] L. Ambrosio, S. Di Marino, and G. Savaré. On the duality between $p$-modulus and probability measures. J. Eur. Math. Soc. (JEMS), 17(8):1817-1853, 2015.

[3] L. Ambrosio, M. Erbar, and G. Savaré. Optimal transport, Cheeger energies and contractivity of dynamic transport distances in extended spaces. Nonlinear Anal., 137:77-134, 2016.

[4] L. Ambrosio and R. Ghezzi. Sobolev and bounded variation functions on metric measure spaces. In Geometry, analysis and dynamics on sub-Riemannian manifolds. Vol. II, EMS Ser. Lect. Math., pages 211-273. Eur. Math. Soc., Zürich, 2016.

[5] L. Ambrosio, N. Gigli, A. Mondino, and T. Rajala. Riemannian Ricci curvature lower bounds in metric measure spaces with $\sigma$-finite measure. Trans. Amer. Math. Soc., 367:4661-4701, 2015.

[6] L. Ambrosio, N. Gigli, and G. Savaré. Gradient flows in metric spaces and in the space of probability measures. Lectures in Mathematics ETH Zürich. Birkhäuser Verlag, Basel, second edition, 2008.

[7] L. Ambrosio, N. Gigli, and G. Savaré. Gradient flows in metric spaces and in the space of probability measures. Lectures in Mathematics ETH Zürich. Birkhäuser Verlag, Basel, second edition, 2008. 
[8] L. Ambrosio, N. Gigli, and G. Savaré. Density of Lipschitz functions and equivalence of weak gradients in metric measure spaces. Rev. Mat. Iberoam., 29(3):969-996, 2013.

[9] L. Ambrosio, N. Gigli, and G. Savaré. Calculus and heat flow in metric measure spaces and applications to spaces with Ricci bounds from below. Invent. Math., 195(2):289-391, 2014.

[10] L. Ambrosio, N. Gigli, and G. Savaré. Calculus and heat flow in metric measure spaces and applications to spaces with Ricci bounds from below. Inventiones Mathematicae, pages 289391, 2014.

[11] L. Ambrosio, N. Gigli, and G. Savaré. Metric measure spaces with Riemannian Ricci curvature bounded from below. Duke Math. J., 163(7):1405-1490, 2014.

[12] L. Ambrosio, N. Gigli, and G. Savaré. Bakry-Émery curvature-dimension condition and Riemannian Ricci curvature bounds. Ann. Probab., 43(1):339-404, 2015.

[13] L. Ambrosio, A. Mondino, and G. Savaré. Nonlinear diffusion equations and curvature conditions in metric measure spaces. ArXiv e-prints, 1509.07273, Sept. 2015.

[14] L. Ambrosio, G. Savaré, and L. Zambotti. Existence and stability for Fokker-Planck equations with log-concave reference measure. Probab. Theory Relat. Fields, 145(3-4):517-564, 2009.

[15] L. Ambrosio and P. Tilli. Topics on analysis in metric spaces, volume 25 of Oxford Lecture Series in Mathematics and its Applications. Oxford University Press, Oxford, 2004.

[16] M. Biroli and U. Mosco. A Saint-Venant principle for Dirichlet forms on discontinuous media. Ann. Mat. Pura Appl., 169:125-181, 1995.

[17] A. Björn and J. Björn. Nonlinear potential theory on metric spaces, volume 17 of EMS Tracts in Mathematics. European Mathematical Society (EMS), Zürich, 2011.

[18] V. I. Bogachev. Gaussian measures, volume 62 of Mathematical Surveys and Monographs. American Mathematical Society, Providence, RI, 1998.

[19] V. I. Bogachev. Measure theory. Vol. I, II. Springer-Verlag, Berlin, 2007.

[20] G. Bouchitte, G. Buttazzo, and P. Seppecher. Energies with respect to a measure and applications to low-dimensional structures. Calc. Var. Partial Differential Equations, 5(1):37-54, 1997.

[21] H. Brezis. Functional analysis, Sobolev spaces and partial differential equations. Universitext. Springer, New York, 2011.

[22] D. Burago, Y. Burago, and S. Ivanov. A course in metric geometry, volume 33 of Graduate Studies in Mathematics. American Mathematical Society, Providence, RI, 2001.

[23] J. Cheeger. Differentiability of Lipschitz functions on metric measure spaces. Geom. Funct. Anal., 9(3):428-517, 1999.

[24] G. Da Prato and J. Zabczyk. Second order partial differential equations in Hilbert spaces, volume 293 of London Mathematical Society Lecture Note Series. Cambridge University Press, Cambridge, 2002. 
[25] C. Dellacherie and P.-A. Meyer. Probabilities and potential, volume 29 of North-Holland Mathematics Studies. North-Holland Publishing Co., Amsterdam, 1978.

[26] S. Di Marino. Sobolev and BV spaces on metric measure spaces via derivations and integration by parts. arXiv e-prints, page arXiv:1409.5620, Sep 2014.

[27] J. Diestel and J. J. Uhl, Jr. Vector measures. American Mathematical Society, Providence, R.I., 1977. With a foreword by B. J. Pettis, Mathematical Surveys, No. 15.

[28] R. E. Edwards. Functional analysis. Dover Publications Inc., New York, 1995. Theory and applications, Corrected reprint of the 1965 original.

[29] R. Engelking. General topology, volume 6 of Sigma Series in Pure Mathematics. Heldermann Verlag, Berlin, second edition, 1989. Translated from the Polish by the author.

[30] M. Erbar, K. Kuwada, and K.-T. Sturm. On the equivalence of the entropic curvature-dimension condition and Bochner's inequality on metric measure spaces. Invent. Math., 201(3):993-1071, 2015.

[31] B. Fuglede. Extremal length and functional completion. Acta Math., 98:171-219, 1957.

[32] M. Fukushima. Regular representations of Dirichlet spaces. Trans. Amer. Math. Soc., 155:455473, 1971.

[33] M. Fukushima, Y. Oshima, and M. Takeda. Dirichlet forms and symmetric Markov processes, volume 19 of de Gruyter Studies in Mathematics. Walter de Gruyter \& Co., Berlin, extended edition, 2011.

[34] E. Gagliardo. A unified structure in various families of function spaces. Compactness and closure theorems. In Proc. Internat. Sympos. Linear Spaces (Jerusalem, 1960), pages 237-241. Jerusalem Academic Press, Jerusalem, 1961.

[35] N. Gigli. On the differential structure of metric measure spaces and applications. Mem. Amer. Math. Soc., 236(1113):vi+91, 2015.

[36] N. Gigli. Lecture notes on differential calculus on RCD spaces. Publ. Res. Inst. Math. Sci., 54(4):855-918, 2018.

[37] N. Gigli. Nonsmooth differential geometry - an approach tailored for spaces with Ricci curvature bounded from below. Mem. Amer. Math. Soc., 251(1196):v+161, 2018.

[38] N. Gigli and E. Pasqualetto. Differential structure associated to axiomatic Sobolev spaces. arXiv e-prints, page arXiv:1807.05417, Jul 2018.

[39] N. Gigli, E. Pasqualetto, and E. Soultanis. Differential of metric valued Sobolev maps. arXiv e-prints, page arXiv:1807.10063, Jul 2018.

[40] N. Gozlan, C. Roberto, and P.-M. Samson. From dimension free concentration to the Poincaré inequality. Calc. Var. Partial Differential Equations, 52(3-4):899-925, 2015.

[41] M. Gromov. Metric structures for Riemannian and non-Riemannian spaces, volume 152 of Progress in Mathematics. Birkhäuser Boston, Inc., Boston, MA, 1999. Based on the 1981 French original [ MR0682063 (85e:53051)], With appendices by M. Katz, P. Pansu and S. Semmes, Translated from the French by Sean Michael Bates. 
[42] P. Hajł asz. Sobolev spaces on an arbitrary metric space. Potential Anal., 5(4):403-415, 1996.

[43] J. Heinonen. Nonsmooth calculus. Bull. Amer. Math. Soc. (N.S.), 44(2):163-232, 2007.

[44] J. Heinonen, T. Kilpeläinen, and O. Martio. Nonlinear potential theory of degenerate elliptic equations. Oxford Mathematical Monographs. The Clarendon Press, Oxford University Press, New York, 1993. Oxford Science Publications.

[45] J. Heinonen and P. Koskela. Quasiconformal maps in metric spaces with controlled geometry. Acta Math., 181(1):1-61, 1998.

[46] J. Heinonen, P. Koskela, N. Shanmugalingam, and J. T. Tyson. Sobolev spaces on metric measure spaces, volume 27 of New Mathematical Monographs. Cambridge University Press, Cambridge, 2015. An approach based on upper gradients.

[47] P. Koskela and P. MacManus. Quasiconformal mappings and Sobolev spaces. Studia Math., 131(1):1-17, 1998.

[48] K. Kuwada. Gradient estimate for Markov kernels, Wasserstein control and Hopf-Lax formula. In Potential theory and its related fields, RIMS Kôkyûroku Bessatsu, B43, pages 61-80. Res. Inst. Math. Sci. (RIMS), Kyoto, 2013.

[49] M. Liero, A. Mielke, and G. Savaré. Optimal entropy-transport problems and a new HellingerKantorovich distance between positive measures. Invent. Math., 211(3):969-1117, 2018.

[50] G. G. Lorentz. Bernstein polynomials. Chelsea Publishing Co., New York, second edition, 1986.

[51] J. Lott and C. Villani. Ricci curvature for metric-measure spaces via optimal transport. Ann. of Math. (2), 169(3):903-991, 2009.

[52] G. Luise and G. Savaré. Contraction and regularizing properties of heat flows in metric measure spaces. ArXiv eprints, 1904.09825, 2019.

[53] Z.-M. Ma and M. Röckner. Introduction to the Theory of (Non-symmetric) Dirichlet Forms. Springer, New York, 1992.

[54] J. R. Munkres. Topology. Prentice Hall, Inc., Upper Saddle River, NJ, 2000. Second edition of [ MR0464128].

[55] E. Paolini and E. Stepanov. Decomposition of acyclic normal currents in a metric space. $J$. Funct. Anal., 263(11):3358-3390, 2012.

[56] W. Rudin. Functional analysis. International Series in Pure and Applied Mathematics. McGrawHill, Inc., New York, second edition, 1991.

[57] L. Schwartz. Radon measures on arbitrary topological spaces and cylindrical measures. Published for the Tata Institute of Fundamental Research, Bombay by Oxford University Press, London, 1973. Tata Institute of Fundamental Research Studies in Mathematics, No. 6.

[58] N. Shanmugalingam. Newtonian spaces: an extension of Sobolev spaces to metric measure spaces. Rev. Mat. Iberoamericana, 16(2):243-279, 2000. 
[59] S. Simons. Minimax and monotonicity, volume 1693 of Lecture Notes in Mathematics. SpringerVerlag, Berlin, 1998.

[60] P. Stollmann. A dual characterization of length spaces with application to Dirichlet metric spaces. Polska Akademia Nauk. Instytut Matematyczny. Studia Mathematica, 198(3):221-233, 2010.

[61] K.-T. Sturm. Analysis on local Dirichlet spaces. II. Upper Gaussian estimates for the fundamental solutions of parabolic equations. Osaka Journal of Mathematics, 32(2):275-312, 1995.

[62] K.-T. Sturm. On the geometry of metric measure spaces. I. Acta Math., 196(1):65-131, 2006.

[63] K.-T. Sturm. On the geometry of metric measure spaces. II. Acta Math., 196(1):133-177, 2006.

[64] C. Villani. Topics in optimal transportation, volume 58 of Graduate Studies in Mathematics. American Mathematical Society, Providence, RI, 2003.

[65] C. Villani. Optimal transport. Old and new, volume 338 of Grundlehren der Mathematischen Wissenschaften. Springer-Verlag, Berlin, 2009. 